\title{
Computational approaches for sub-meter ocean color remote sensing
}

\author{
by \\ Ryan Edward O'Shea \\ B.S., University of Rhode Island (2015) \\ Submitted to the Department of Mechanical Engineering \\ in partial fulfillment of the requirements for the degree of \\ Doctor of Philosophy in Mechanical and Oceanographic Engineering \\ at the \\ MASSACHUSETTS INSTITUTE OF TECHNOLOGY \\ and the \\ WOODS HOLE OCEANOGRAPHIC INSTITUTION \\ February 2021 \\ (C)2021 Ryan E. O'Shea. All rights reserved.
}

The author hereby grants to MIT and WHOI permission to reproduce and to distribute publicly paper and electronic copies of this thesis document in whole or in part in any medium now known or hereafter created.

Author

Joint Program in Oceanography / Applied Ocean Science \& Engineering Massachusetts Institute of Technology \& Woods Hole Oceanographic Institution

December 2, 2020

Certified by

Dr. Samuel R. Laney

Associate Scientist with Tenure, Biology Department

Woods Hole Oceanographic Institution

Thesis Supervisor

Accepted by

Dr. Nicholas Hadjiconstantinou

Professor of Mechanical Engineering

Chair, Department Committee on Graduate Students,

Massachusetts Institute of Technology

Accepted by

Dr. David Ralston

Associate Scientist with Tenure, Applied Ocean Physics \& Engineering Department

Chair, Joint Committee for Applied Ocean Science \& Engineering

Woods Hole Oceanographic Institution 


\title{
Computational approaches for sub-meter ocean color remote sensing
}

\author{
by \\ Ryan Edward O'Shea \\ Submitted to the Department of Mechanical Engineering \\ on December 2, 2020, in partial fulfillment of the \\ requirements for the degree of \\ Doctor of Philosophy in Mechanical and Oceanographic Engineering
}

\begin{abstract}
The satellite ocean color remote sensing paradigm developed by government space agencies enables the assessment of ocean color products on global scales at kilometer resolutions. A similar paradigm has not yet been developed for regional scales at sub-meter resolutions, but it is essential for specific ocean color applications (e.g., mapping algal biomass in the marginal ice zone). While many aspects of the satellite ocean color remote sensing paradigm are applicable to sub-meter scales, steps within the paradigm must be adapted to the optical character of the ocean at these scales and the opto-electronics of the available sensing instruments. This dissertation adapts the three steps of the satellite ocean color remote sensing paradigm that benefit the most from reassessment at sub-meter scales, namely the correction for surface-reflected light, the design and selection of the opto-electronics, and the post-processing of over-sampled regions. First, I identify which surface-reflected light removal algorithm and view angle combination are optimal at sub-meter scales, using data collected during a field deployment to the Martha's Vineyard Coastal Observatory. I find that of the three most widely used glint correction algorithms, a spectral optimization based approach applied to measurements with a $40^{\circ}$ view angle best recovers the remotesensing reflectance and chlorophyll concentration despite centimeter scale variability in the surface-reflected light. Second, I develop a simulation framework to assess the impact of higher optical and electronics noise on ocean color product retrieval from unique ocean color scenarios. I demonstrate the framework's power as a design tool by identifying hardware limitations, and developing potential solutions, for estimating algal biomass from high dynamic range sensing in the marginal ice zone. Third, I investigate a spectral super-resolution technique for application to spatially over-sampled oceanic regions. I determine that this technique more accurately represents spectral frequencies beyond the Nyquist and that it can be trained to be invariant to noise sources characteristic of ocean color remote sensing on images with similar statistics as the training dataset. Overall, the developed and critically assessed sub-meter ocean color remote sensing paradigm enables researchers to collect high fidelity sub-meter data from imaging spectrometers in unique ocean color scenarios.
\end{abstract}

Thesis Supervisor: Dr. Samuel R. Laney

Title: Associate Scientist with Tenure, Biology Department

Woods Hole Oceanographic Institution 


\section{Acknowledgments}

First and foremost, thank you to my advisor Dr. Samuel Laney for supporting me through my failures and successes. It was a strenuous journey, but with your support I have grown more professionally than I ever thought possible. Second, I would like to thank my committee members for providing targeted feedback that greatly improved the direction and content of my thesis. Of the committee members, I would particularly like to thank the chair of my committee, Dr. George Barbastathis, for allowing me to be a part of the 3D Optics Lab Group at MIT.

For technical contributions to the research I'd like to thank H. Popenoe, J. Sisson, E. Bates, Z. Sandwith, E. Timmons, the Sosik Lab (especially T. Crockford and E. Peacock), Maria T. Kavanaugh, and Jennie E. Rheuben.

To the JP Jellyfish, thank you all so much for providing such a supportive community. I am particularly grateful for the memories made with Nathan, EeShan, Jeffrey, Chrissie, Rachel, Scott, Billy, and Jake. I will always have fond memories of Dokupon, OGB, DoTA, flag football, ultimate frisbee, apple picking, rock climbing, and DND.

To my brother and sister, Andrew and Sabrina, thank you for always being willing to hang out on the drop of a hat when I needed it most, providing me with distractions via gaming and binge watching TV series, and listening to my many complaints.

To my partner, Lizzie, thank you for supporting me and keeping me positive.

And finally to my parents, thank you for your continuing love and support. Dad, I'm starting to think you had a long-term plan when you re-watched Armageddon and Independence Day with me for the nth time. Mom, thank you for instilling an appreciation for education and hard work in me.

Ryan O'Shea was supported by the Department of Defense (DoD) through the National Defense Science \& Engineering Graduate Fellowship (NDSEG) Program. This research was funded by Woods Hole Oceanographic Institution's Edwin W. Hiam Ocean Science and Technology Award Fund, its Ocean Venture Funds, its Academic Programs Office, and the National Aeronautics and Space Administration via grant number CCE NNX17AI72G to Dr. Samuel Laney.

The raw data for Figures 3-3 and 3-4 were provided through Australian Antarctic Science grants 2678 and 4390 . 


\section{Contents}

\begin{tabular}{lll}
\hline & Introduction & 27
\end{tabular}

$1.1 \quad$ Evolution of the Satellite Ocean Color Remote Sensing Paradigm . . . . . . . 28

1.2 The Current Satellite Ocean Color Remote Sensing Paradigm . . . . . . . . . 31

1.2.1 Insolation of the Ocean's Surface: Fundamental Physics \& Atmospheric Optics . . . . . . . . . . . . . . . 35

1.2.2 Transmission and Reflection at the Ocean's Surface: Classical Optics . . . . . . . . . . . . . . . . . . . . 37

1.2.3 Absorption, Scattering, and Fluorescence in the Water Column: Ocean Optics . . . . . . . . . . . . . . . . . . 38

$1.2 .4 \quad$ Measuring Ocean Color: Spectral Imaging . . . . . . . . . . . . . . . . 41

1.2.5 Signal Detection: Photonics \& Optoelectronics . . . . . . . . . . . . . 42

1.2.6 Mitigating Optical Errors: Optical Engineering \& Information Processing . . . . . . . . . . . . . . . . . . . . 44 44

1.2 .7 Ocean Color Algorithms: Information Acquisition . . . . . . . . . . . 46

1.3 Challenges in Ocean Color Remote Sensing on Meter Scales: Limitations of the Satellite Paradigm . . . . . . . . . . . . . . . . . . . . . . 49

1.3.1 Spatio-temporal limitations of Satellite Deployment Approaches . . . . 50

1.3.2 Overview of Non-Satellite Platforms for Ocean Color Measurement . . 52

1.3.3 Observational Considerations . . . . . . . . . . . . . . . 55

$1.3 .4 \quad$ Camera and Imaging Considerations . . . . . . . . . . . . . . . . 55

1.3.5 Calibration and Testing Considerations _. . . . . . . . . . 57

1.3.6 Environmental Considerations . . . . . . . . . . . . . . . . . . . . 58

1.3.7 State-of-the-Art for Sub-Orbital Optical Constituent Mapping. . . . . 59

1.3.8 Identified Limitations in Fine-scale Optical Constituent Estimation . . 63 
1.4 Dissertation Overview $\ldots \ldots \ldots \ldots \ldots$

2 Evaluation of glint correction approaches for fine-scale ocean color measurements by lightweight hyperspectral imaging spectrometers $\quad 69$

2.1 Introduction $\ldots \ldots \ldots \ldots \ldots \ldots \ldots$

2.1 .1 Motivation . . . . . . . . . . . . . . . . . . 70

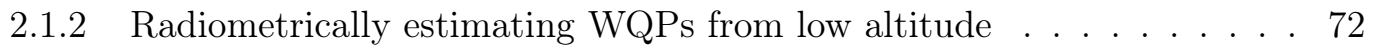

$2.1 .3 \quad$ Spectral signal-to-noise ratio considerations . . . . . . . . . . . 75

2.1 .4 Correcting for sun and sky glint within a single image . . . . . . . . 77

2.2 Materials and Methods . . . . . . . . . . . . . . . . . . . . . . 80

$2.2 .1 \quad$ A representative lightweight imaging spectrometer . . . . . . . . . . . 80

$2.2 .2 \quad$ Laboratory calibration of imaging spectrometer . . . . . . . . . . . . . 81

$2.3 \quad$ Field assessment: fixed-platform study . . . . . . . . . . . . . . . . . . . . 82

$2.3 .1 \quad$ Imaging spectrometer: observational geometry . . . . . . . . . . . . 84

$2.3 .2 \quad$ Ancillary above-water instruments and measurements . . . . . . . . 84

$2.3 .3 \quad$ In situ time series of $[\mathrm{chl}]$ and $\mathrm{b}_{\mathrm{bp} 650} \ldots \ldots \ldots \ldots$. . . . . . . . 85

$2.3 .4 \quad$ Algorithms used for WQP estimation: [chl] and $b_{\mathrm{bp} 650}$. . . . . . . . . 85

$2.3 .5 \quad \mathrm{R}_{\mathrm{rs}}$ Quality Assurance $\ldots \ldots \ldots \ldots$. . . . . . . . . . . . 88

2.4 Results . . . . . . . . . . . . . . . . . . . . . . . . . . . . . . . . 88

$2.4 .1 \quad$ Glint correction approaches and radiometric precision . . . . . . . 88

$2.4 .2 \quad$ Glint correction approaches and WQP estimate accuracy . . . . . . . . 90

2.5 Discussion $\ldots \ldots \ldots \ldots \ldots$

2.5.1 Glint correction in a fine-scale ocean imaging context . . . . . . . . . . 98

2.5 .2 Implications of view angle on glint correction . . . . . . . . . . . . 100

2.5.3 Practical factors for glint removal from mobile platforms . . . . . . . . 101

2.6 Conclusions $\ldots \ldots \ldots \ldots$

3 A simulation framework for evaluating lightweight spectral cameras in $\begin{array}{ll}\text { drone-based aquatic sensing applications } & 105\end{array}$

$3.1 \quad$ Introduction . . . . . . . . . . . . . . . . . . . . . . . . . . . . . . . . 105

3.2 Overview of Key Camera Parameters and Environmental Considerations . . . 108

3.3 Representing Drone-based Hyperspectral Remote Sensing Scenarios in A Sim-

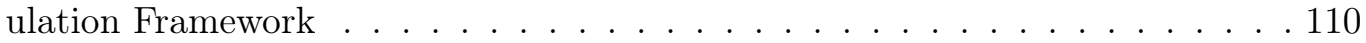


3.3 .1 Ocean color formation . . . . . . . . . . . . . . . . . . 111

3.3.2 Optical propagation through the atmosphere into a camera system at altitude . . . . . . . . . . . . . . . . . . 112

$3.3 .3 \quad$ Transmission through an optical train . . . . . . . . . . . . . . . . . . 114

3.3 .4 Application of noise . . . . . . . . . . . . . . . . . . 115

3.3.5 Implementation of glint correction algorithms . . . . . . . . . . . . 116

3.3 .6 Estimation of OAC concentrations . . . . . . . . . . . . . . . . . . 117

3.3.7 Comparing simulated to known OAC concentrations: statistics . . . . 118

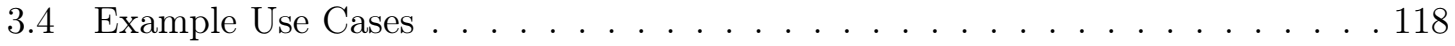

3.4.1 Modeling the required radiometric resolution of cameras to remotely estimate chlorophyll FLH $\ldots$. . . . . . . . . . . . . . . . . . . . 118

3.4.2 Determining if a NIR-blazed grating reduces the minimum achievable MAPD for measuring chlorophyll FLH . . . . . . . . . . . . . . . . . . 120

3.4.3 Evaluating the trade-off between spatial resolution and accuracy for the remote estimation of pelagic chlorophyll between spatially heterogeneous Antarctic sea ice floes . . . . . . . . . . . . . . . . . . . . . . 121

3.4.4 Estimating the full-well capacity imposed limitations on remotely estimating algal biomass in Antarctic waters . . . . . . . . . . . . . . 123

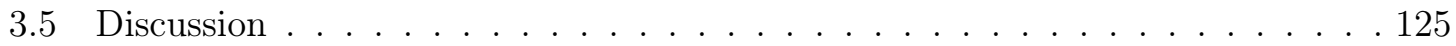

3.6 Conclusion $\ldots \ldots \ldots \ldots \ldots$

4 Spectral super-resolution via spectral shift in spatially oversampled images: Considerations for sub-meter ocean color remote sensing $\quad 131$

4.1 Introduction . . . . . . . . . . . . . . . . . . . . . . . 131

4.2 State-of-the-art spectral super-resolution techniques . . . . . . . . . . . . 133

4.3 Application to Sub-meter Ocean Color Remote Sensing: Motivation And Lim-

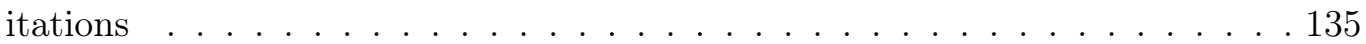

4.4 Methods . . . . . . . . . . . . . . . . . . . . . . . . . . . . 136

$4.4 .1 \quad$ Sources of Sub-Pixel Spectral Shift in Grating Based Imaging Spectrometers . . . . . . . . . . . . . . . . 136

4.4.2 Dataset collection: Images with sub-pixel shift and associated truth spectra . . . . . . . . . . . . . . . . . . . 137 
$4.4 .3 \quad$ Machine Learning Optimization . . . . . . . . . . . . . . . . . . 141

4.4 .4 Setting the Signal-to-Noise Ratio with Gaussian Noise . . . . . . . . . 141

4.4.5 Simulating Residual Glint on the Laboratory Collected Dataset . . . . 142

4.5 Results $\ldots \ldots \ldots \ldots \ldots$. . . . . . . . . . . . . . . . . . . . . . . . . . . . . . . . . . . . 144

4.5.1 Leveraging sub-pixel spectral shift in homogeneous images for increased spectral resolution . . . . . . . . . . . . . . . . . . . . . . . . . 144

4.5.2 Generalization of SVRs for spectral super-resolution: ocean color signal, band-pass/band-stop, and real world signals . . . . . . . . . . . . 146

4.5.3 Impact of noise on super-resolution: Generalization of noisy SVRs to laboratory generated ocean color, band-pass/band-stop, and realworld signals . . . . . . . . . . . . . . . . . . . . . . . . . . 149

4.5.4 Spatial subsets for spectral super-resolution . . . . . . . . . . . . 153

4.6 Discussion $\ldots \ldots \ldots \ldots$

4.6.1 Sub-pixel spectral shift enables spectral super-resolution on datasets with similar statistics . . . . . . . . . . . . . . . . . . . . . 154

4.6.2 SVRs can be trained to be insensitive to environmental and optical noise typical of sub-meter ocean color images . . . . . . . . . . . . . . 154

4.6.3 SS SVRs weakly generalize to datasets with different statistics. . . . . 155

4.6.4 Comparison to alternative spectral super-resolution approaches … . 157

4.6 .5 Implications for adaptive sensing of the ocean's color . . . . . . . . . . 157

4.6 .6 Limitations of the laboratory based study and future work . . . . . . . 158

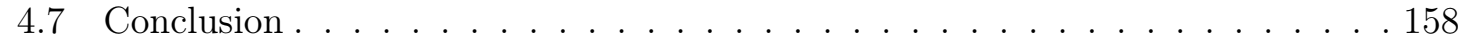

$\begin{array}{lll}5 \text { Conclusion } & 161\end{array}$

5.1 Dissertation's Contributions to Sub-meter Ocean Color Remote Sensing . . . 161

5.1 .1 Chapter 2 Contributions . . . . . . . . . . . . . . . . . . . . 161

5.1 .2 Chapter 3 Contributions . . . . . . . . . . . . . . . . . 162

$5.1 .3 \quad$ Chapter 4 Contributions $\ldots . \ldots \ldots 2$

$5.1 .4 \quad$ A New Paradigm for Sub-Meter Ocean Color Mapping in Spatially Heterogeneous Regions . . . . . . . . . . . . . . . . . . . 163

$5.2 \quad$ Future Work in Sub-Meter Ocean Color Remote Sensing . . . . . . . . . . . . 167

5.3 Applications of Dissertation Research Beyond Ocean Color Remote Sensing . 170 
\begin{tabular}{|ll|}
\hline A Determining the analog-to-digital conversion factor & 173
\end{tabular}

\begin{tabular}{ll}
\hline B Comparing theoretically and spatially derived SNRs & 175
\end{tabular}

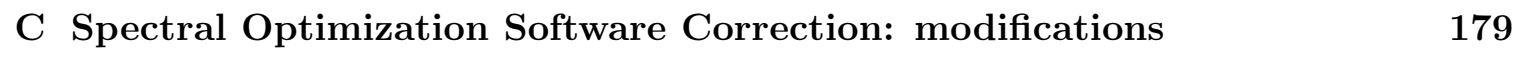




\section{List of Figures}

1-1 The ocean color remote sensing paradigm leverages knowledge from a wide range of optical disciplines (1-10) to retrieve the optical constituent products from a measured spectrum. In this paradigm, light from the sun (1) propagates through the atmosphere (2) and ocean surface (3), before being scattered by optical constituents (4) to a sensor at altitude (5-8). Then the measured electrons are converted to a reflectance value $(\mathbf{9})$ and the environmental noise sources are estimated and removed. Finally, the optical constituent products are calculated (10). . . . . . . . . . . . . . . . 34

1-2 Physical and biological processes within the global oceans occur over a wide range of spatio-temporal resolution combinations. Satellites are able to collect global datasets over decadal timespans, but only at coarse resolutions. Aircraft are able to sense at finer spatial and temporal resolutions, but at the cost of limited temporal and spatial coverage. Drones can sense at submeter spatial scales, but sub-meter fluctuations in the surface characteristics (yellow circle) mar the radiometric data at these scales. This figure was adapted from IOCCG Report No. 12 |International Ocean-Colour Coordinating Group $[2012 \mathrm{~b} \mid$ which was itself adapted from Dickey et al. |Dickey

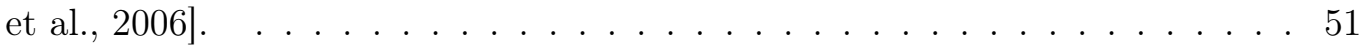


2-1 Center wavelengths used in common algorithms for various WQPs |Lee et al., 2010; Mobley, 1999:[Mueller and Austin,[1995[]Shahraiyni et al.,[2007; Keith et al.,[2014][Ekercin,]2007[]Woźniak et al., 2018[] Gurlin et al.,[2011][O'Reilly et al. [1998[ Gholizadeh et al. [2016]. Highest accuracy algorithms often utilize a larger number of wavelengths. The gap in the waveband set for Lee10 (a glint correction algorithm) indicates wavelengths omitted surrounding the chlorophyll fluorescence emission. Abbreviations: CDOM - colored dissolved organic matter; $\mathrm{b}_{\mathrm{b} 620}$ - optical backscatter at $620 \mathrm{~nm}$; SPM - suspended particulate material; [chl] - chlorophyll concentration. . . . . . . . . . . . 71

2-2 Suspended particulate matter (SPM), colored dissolved organic matter (CDOM), and phytoplankton are optically active WQPs that shape the water-leaving radiance $\left(\mathrm{L}_{\mathrm{W}}\right)$ in optically deep water bodies. Accurately estimating their concentrations from a ratio of the total at-sensor spectral radiance $\left[\mathrm{L}_{\mathrm{T}}(\mathrm{h})\right]$ and the downwelling solar spectral irradiance $\left[\mathrm{E}_{\mathrm{d}}(\mathrm{h})\right]$ is complicated by environmental noise sources including the path radiance $\left(\mathrm{L}_{\text {path }}\right)$, reflected solar glint $\left(\mathrm{L}_{\mathrm{S}}\right)$, and reflected sky radiance $\left(\mathrm{L}_{\mathrm{sky}}\right)$. On fine spatial scales, glint is strongly affected by sea state and wave characteristics. . . . . . . . . . . . . . 72

2-3 The imaging spectrometer and ancillary instruments (right) as deployed at the MVCO ASIT (left). Numbers marking the various components are referenced in Table $2.2 \ldots \ldots \ldots \ldots \ldots$. . . . . . . . . . . . . . . . . . . . 82

2-4 Environmental conditions over the 2.5 month field assessment study. Top panel: wind speed. Second panel: incident solar irradiance at the ASIT. Third panel: daily in situ $[\mathrm{chl}]$. Bottom panel: $\mathrm{b}_{\mathrm{bp} 650}$. . . . . . . . . . . . . . 86

2-5 Top panel: Water-leaving radiances during high [chl] conditions were maxi-

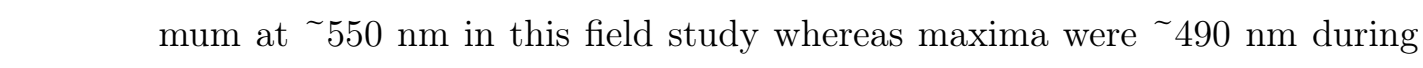
\begin{tabular}{|c|}
\hline low [chl] conditions. Conditions of high downwelling irradiance (i.e., bright \\
\hline
\end{tabular} sunlight) corresponded with greater upwelling radiances. The shape of the SNR is comparable to its associated radiance, with higher SNRs lining up

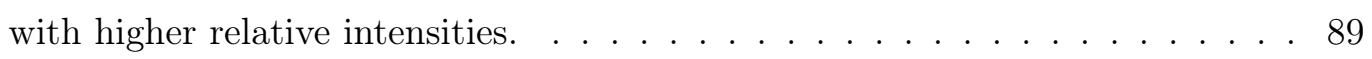


2-6 The median (theoretically-derived) SNR as calculated over the entire time series. Highest SNRs are in the green section of the spectrum for both spectra. Use of a polarizer decreased the SNR in the red and part of the NIR. The uncorrected SNR data represent a range of view angles between 39.75-42.70 and the polarizer-corrected SNR is calculated from a range of view angles

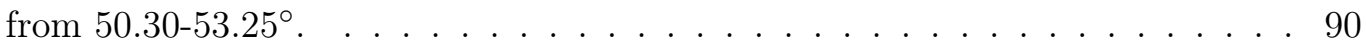

2-7 The median $\mathrm{R}_{\mathrm{rs}}$ and standard deviation for the entire time series, as a function of wavelength. Each glint correction approach reduces the median $\mathrm{R}_{\mathrm{rs}}$ (top panel) and decreases the SD towards the shot noise limited theoretical value (bottom panel) relative to the uncorrected ' $\mathrm{R}_{\mathrm{rs}}$ ' (in which the total radiance approximates the water leaving radiance). . . . . . . . . . . . . . . . 91

2-8 Training data relationships between in situ measured (abscissa) and radiometricallyestimated (ordinate) WQPs of chlorophyll (top panel) and $\mathrm{b}_{\mathrm{bp650}}$ (bottom \begin{tabular}{|l|l|l|l|l|l|}
\hline panel) using Eqs. & 2.4 & and & 2.5 & respectively. The SOSC glint correction ap- \\
\hline
\end{tabular}

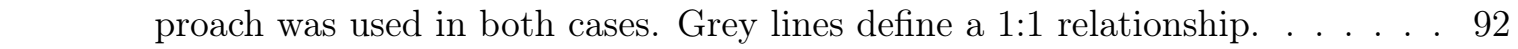

2-9 An example of hyperspectral $\mathrm{R}_{\mathrm{rs}}$ data taken with the candidate imager first uncorrected (top panel) and after applying the SOSC approach (bottom panel). An example spatial distribution of remotely estimated [chl] that would be interpreted from these data is presented at the top of each panel. Artifacts due to glint can clearly be seen as bright streaks across all wavelengths at specific distances in the uncorrected images (arrows). Left uncorrected this introduces substantial spatial variability in the estimated [chl] that is solely an artifact of variations in the glint. Here, the SOSC approach reduces this artifact considerably. The in situ $[\mathrm{chl}]$ is $8.6 \mu \mathrm{g} \cdot \mathrm{l}^{-1}$. . . . . . . . . . . . . . . 93

2-10 The median MAPE between estimates of [chl] and $\mathrm{b}_{\mathrm{bp} 650}$ as determined from radiometric algorithms. Measurements from a nonimaging hyperspectral radiometer (circles) were made at only one view angle $\left(45^{\circ}\right)$ whereas the vertical orientation of this imaging spectrometer in our deployment allowed us to examine MAPE along a broader range of view angles. Notations in the top panel indicate which trace relates to each glint correction algorithm. The mean Wei et al. $\mid$ Wei et al. $\left[2016 \mid\right.$ quality assurance (QA) of the $\mathrm{R}_{\mathrm{rs}}$ generally demonstrates an inverse relationship with the MAPE of the WQP estimates. 95 
2-11 The MAPE of median daily estimates of [chl] and $b_{\mathrm{bp} 650}$, binned across the dynamic range observed in the field study. Numbers above each bin indicate the median number of data points used. The dashed line indicates the $35 \%$ [chl] accuracy goal for the SeaWiFS satellite sensor [McClain et al.[|1998]. . . 97

3-1 Top: the optical propagation of water-leaving radiance through a drone-based hyperspectral imaging system. The water-leaving radiance (A) first propa-

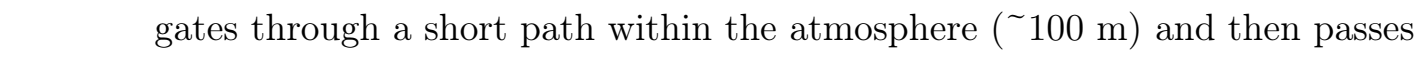
into the imaging optics of an airborne sensor at a given altitude (B). This radiance is then transmitted through the imaging optics of the camera system (C) and imaged by the focal plane array (FPA), which adds optical and electronic noise to the signal (D). If desired, an algorithm can be applied to correct for glint artifacts before computing the measured, noisy, total water-leaving radiances at different wavelengths (E). These are then used in ocean color algorithms to compute any given OAC of interest $(\mathrm{F})$. The overall effect of the camera system noise can be determined by comparing these remote sensing estimates to the 'truth' values used to initiate this simulation $(\mathrm{G})$, which are derived either from in-situ measurements or input data from aquatic optical modeling tools such as Hydrolight (Sequoia Scientific |Mobley and Sundman, 2008|). Bottom: the mapping of this physical description onto the simulation framework. Grey text indicates selectable parameters of camera systems that affect each step. . . . . . . . . . . . . . . . . . . . . . 113

3-2 The median median absolute percent difference (MAPD) between the optically noisy and noiseless fluorescence line height (FLH) estimate is shown as a function of the radiometric resolution (bit count) and grating blaze type. The VIS-blazed line shows the MAPD when using a visible blazed grating, and the NIR-blazed line shows the MAPD when using a NIR blazed grating instead. . . . . . . . . . . . . . . . . . . . . 120 
3-3 An example RGB image of sea ice in the Weddell Sea (panel A), with dimensions of $127 \mathrm{~m}$ by $82 \mathrm{~m}$ (plotted from raw data provided by Steer et al. |Steer et al. [2008]). Pixels representing open water (panel B, black points within red circle) were identified by thresholding the red intensity and blue to red ratio. Additional thresholding can identify areas of algal presence within the sea ice itself (panel C) through the ratio of blue to red light. . . . . . . . . 123

3-4 The median filtered fraction of the the total water that is imaged without ice contributions as a function of sea ice concentration and spatial resolution for 250 images in high sea ice cover [Steer et al.| 2008|. The dark grey and black dots correspond to 0.8 meter and 1.6 meter resolutions, the chlorophyll estimation accuracy at these resolutions is estimated in Fig. $\mid 3-5\rceil$. . . . . . . . . 124

3-5 The median MAPD and standard deviation (error bars) from the Mueller and Austin |Mueller and Austin, $[1995 \mid$ corrected data are plotted versus the along-track spatial resolution (a function of exposure time) of a drone flying at $100 \mathrm{MPH}$, the maximum FAA limited speed |Federal Aviation Administration (FAA),$[2016 \mathrm{a} \mid$. The dark grey and black dots correspond to the spatial resolutions selected in Fig. $\mid 3-4$, and show a trade-off in fraction of water area represented and MAPD. The light grey dot shows the high error imaging the sea ice algae imposes on the pelagic chlorophyll estimates. The ordinate axis uses log-space to increase visual clarity by separating the error bars at the lowest spatial resolutions. . . . . . . . . . . . . . . . . . . . . . . 125

4-1 The interface between a river with high colored dissolved organic matter (CDOM) and the coastal ocean, dominantly containing green algae, in northern Alaska. High spatial resolution images are required to sense the interface (grey bar), while the homogeneous waters in the high CDOM river (brown bar, left) and coastal ocean waters (green bar, right) are over-sampled. Photo credit: Luka Ćatipović. . . . . . . . . . . . . . . . . . . 136 
4-2 In an ideal image of a homogeneous target made from a imaging spectrometer, the vertical position of any individual spectra does not vary as a function of spatial location (A). The sub-pixel spectral shift required for super-resolution can be induced in ideal imagers by tilting the focal plane array (FPA) relative to the optical axis (B, red box), or can occur in non-ideal imagers suffering from the spectral curvature effect ('smile', C) when measured by an on-axis FPA $(\mathrm{D}, \mathrm{red}$ box $) . \ldots \ldots \ldots \ldots$

4-3 A 20 Watt Tungsten Halogen source provides white light as input to a digital monochromator. The monochromator shifts wavelengths after random amounts of time, to generate a randomly generated spectral output. The light passes through a 50 micrometer bifurcated fiber, which allows the same input light to pass through a 'truth' spectrometer with fine spectral resolution (0.7 nm FWHM of spectral blur, $0.2 \mathrm{~nm}$ sampling) and a 'test' imaging spectrometer with coarse (1.7 nm FWHM of spectral blur, $2.1 \mathrm{~nm}$ sampling) spectral resolution. The instruments are hardware triggered so that the measure the same input signal. . . . . . . . . . . . . . . . . . . . . . . . . 138

4-4 The spectral pixel that measures a monochromatic light source (e.g. 702.2 $\mathrm{nm}$, top curve) is plotted for each spatial location, for 25 different central wavelengths. The spatial dependence, which is similar in shape to a quadratic characteristic of smile and a linear trend from a shifted focal plane array, holds across the wavelength range. The technique fails at low SNRs, which occur above spatial pixel 700 (right side). The median change between the highest spectral wavelength and the lowest spectral wavelength (from spatial pixel 1-700) is 1.7 pixels, which corresponds to $\sim 1.8 \mathrm{~nm}$ of spectral change in the reported wavelength over the spatial domain. . . . . . . . . . . . . . . . . 140

4-5 An example cm scale hyperspectral image of homogeneous oceanic waters suffering from residual glint correction errors (top, spectrally flat offsets near 43 and 51 degrees) O'Shea et al. [2020|. The distribution of radiance at 505 $\mathrm{nm}$ normalized by the median value is non-Gaussian and includes significant offsets (bottom). . . . . . . . . . . . . . . . . . . . . 143 
4-6 Example training and validation MAPD between the spectrometer measured intensity and the SVR estimated intensity from homogeneous images with $1.8 \mathrm{~nm}$ of spectral shift (SS) and on a SVR trained on data without spectral shift (NSS) for one spectral output. Both SS and NSS SVRs converge within 15,000 iterations. The SS SVR achieves a much higher validation accuracy $(\sim 4 \%)$ than the NSS SVR $(\sim 33 \%) . \ldots \ldots \ldots$

4-7 Example predictions for training and testing datasets. The SVRs trained on data including spectral shift (SS) match the low and high frequency fluctuations in the normalized intensity measurements from the Ocean Optics spectrometer (OOS Truth) values (black) better than either the SVRs trained on data without spectral shift (NSS, teal) or the median of the NSS data rescaled to the normalized intensity values (RS, blue, asterisks are pixel center wavelengths). . . . . . . . . . . . . . . . . . . . . . 146

4-8 The single sided power spectrum of a prediction from the testing set using the SS SVRs (red, top panel) matches the truth data (black) across the spatial frequency domain, even past the pixel limited Nyquist frequency (NF, dark grey bars). The NSS SVRs (teal) match the frequency content until just before the Nyquist frequency, while the re-scaled (RS) raw pixel data (blue) only matches the spectral content at the lowest spatial frequencies. The median absolute percent difference between spectral content of the SS, NSS, and RS predictions and the OOS Truth values over the entire testing set demonstrate the benefit of the SS at all spectral frequencies (bottom panel). 147

4-9 Example spectra produced from testing data with a variety of different statistics including laboratory generated band-stop (BS), band-pass (BP), and ocean color (OC) and two short-pass transmission filter's (Filter) straight on (Straight) and at an angle (Angled). Generally, the SS SVRs can capture medium frequency spectral features in these signals at the cost of noise in the higher frequencies. . . . . . . . . . . . . . . . . . . . . . . 148 
4-10 The single sided power spectrum of the prediction produced from the SS SVRs (red, top panel) on an example laboratory generated ocean color signal (Fig. $4-9$ F) matches the truth spectrum (black) at low frequencies, even slightly past the pixel limited Nyquist frequency (dark grey dot dashed line), but poorly represents higher frequency content, overestimating at mediumhigh frequencies. The NSS SVRs (teal) match the frequency content until half of the Nyquist frequency, while the re-scaled raw pixel data (blue) only matches the spectral content at the lowest spatial frequencies and underestimates higher spectral frequencies. The median absolute percent difference (MAPD) calculated between the OOS Truth measurements spectral content and the spectral content of the SS, NSS, and RS predictions for all of the generated ocean color signals demonstrates the benefit of the SS SVRs at accurately representing the low spectral frequencies surrounding the Nyquist (bottom panel). . . . . . . . . . . . . . . . . . 150

5-1 The individual chapters combine to increase the fidelity of the measurements

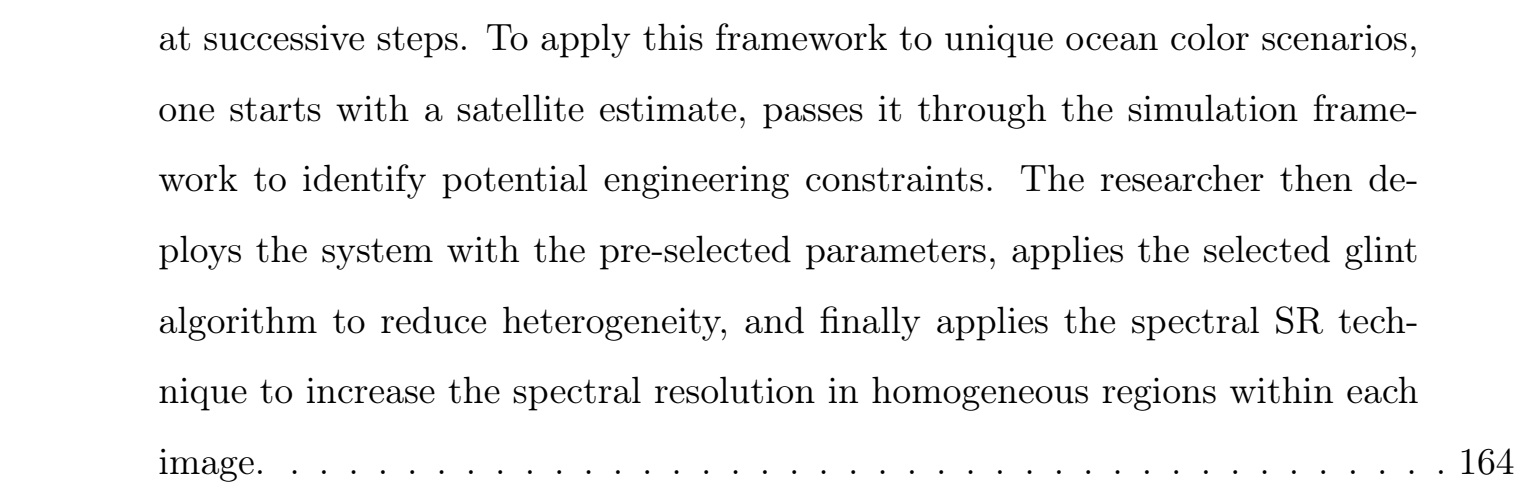

5-2 The spatial filtering techniques provide a slight reduction in the median abso\begin{tabular}{|cc}
\hline lute percent difference in the estimated chlorophyll concentrations relative to \\
\hline
\end{tabular} the uncorrected dataset, but sacrifice significant spatial resolution (from ${ }^{2}$ $\mathrm{cm}$ to $\sim 35 \mathrm{~cm}$ resolution) to do so. The spatial correction techniques perform worse than all of the spectral glint correction techniques across the entire view angle range. The results show slightly different values than previously presented view-angle dependent results (Fig. 2 -10), as MAPD is used instead of MAPE, and the error estimate was performed on a slightly different testing dataset. . . . . . . . . . . . . . . . . . . . 168 
B-1 When no radiometric correction technique is applied (Spa. 0), the pixel-topixel variations limit the spatial SNR below the theoretical limit (Theo.). When we radiometrically correct using 100 images, the spatially-derived SNR (Spa. 100) and the theoretically derived SNR (Theo.) match. Only one radiometric correction image is used to correct our observational data set. The spatially-derived SNR using 1 correction image (Spa. 1) can be approximated by the theoretical limit due to a single correction image (Theo. CT1) [Eqs.

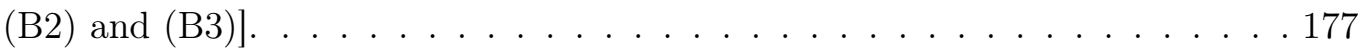




\section{List of Tables}

1.1 The dominant factors that limit sub-meter ocean color remote sensing, where measurements are made from low altitudes with low quality optics, are dramatically different from those at $\mathrm{km}$ spatial resolutions from high altitudes with state-of-the-art optics ${ }^{1,2}[\mathrm{Su},[2017$; $[$ Larson et al. $[2018 ;$ Totsuka et al., 2019: Kwon et al., 2020; Kislik et al. [2018|, ${ }^{3,4} \mid$ Kislik et al., 2018; O'Shea et al. [2020: O'Shea and Laney, 2020|, ${ }^{4} \mid$ Honkavaara et al. $[2013 \mid$ ). This dissertation ameliorates the highest impact factors within chapters 2-4. . . . . . 29

1.2 The available bands and achievable spatial resolutions of the most commonly used satellite-borne ocean color sensors have slowly increased over time. . . . 32

1.3 The increasing radiometric resolution and SNR demonstrate the advancement of the ocean color imager electronics through the ocean color sensor deployments. The lower SNR of HICO is due to the increased spectral resolution.

Full-well depths in parenthesis are estimated based on the maximum stated SNR |Qian, 2015][Lucke et al.] 2011:[Bezy et al.] 1997[ Qimaging]. . . . . . . . 43

1.4 The available drones can be placed into classes based upon their operational capabilities, following a similar classification system as the U.S. Army |U.S. Army UAS Center of Excellence, $[2010 \mid$. This dissertation focuses on the Micro and Miniature classes of drones as they have sufficient payload for ocean color sensors and sufficient endurance for $\mathrm{km}$ scale ocean color missions, but can still be launched by a single researcher. . . . . . . . . . . . . . . . . . . . . 54

1.5 A comparison between the platform induced limitations of satellites, aircraft, and drones. Bold characteristics in the drone column may impact the validity of the satellite remote sensing paradigm on sub-meter estimates. . . . . . 56 
$1.6 \quad$ A comparison between the typical camera characteristics available for each platform. Bold characteristics in the drone column may impact the validity of the satellite remote sensing paradigm on sub-meter estimates. . . . . . . . 57

1.7 The burden of calibration and validation of drone-borne ocean color imagers currently falls upon the ocean color scientist. Requiring the ocean color scientist to calibrate and validate the products will introduce additional uncertainty and reduce productivity relative to the aircraft or satellite calibration model. Bold characteristics in the drone column may impact the validity of the satellite remote sensing paradigm on sub-meter estimates. . . . . . . . . 58

1.8 A comparison between the environmental factors effecting ocean color measurements from satellites, aircraft, and drones. Bold characteristics in the drone column may impact the validity of the satellite remote sensing paradigm on fine-scale estimates. . . . . . . . . . . . . . . . . . . . . . . . . 60

1.9 Airborne ocean color imagers advanced significantly over the past few decades. The size and weight of the airborne imagers has decreased, while their optical efficiency and spectral resolution has dramatically increased. Unfortunately, the airborne ocean color imagers are too large for deployment on the available drone platforms. [Mouroulis et al.] 2014; Kramer; Green et al.] 2001b a: Dickey et al. $[2006[$ Davis $[$ Davis et al. $[\mid 2002] \ldots$. . . . . . . . . . . . . . . 61

2.1 A selection table for a representative set of commercially available imagers suitable for lightweight drones. Asterisks signify imagers whose wavelengths can be factory customized, italics indicate a requirement not met for simultaneous recovery of multiple WQPs, and bold indicates a requirement investigated here. The shot noise limited SNR is calculated from full well capacity data obtained from the vendor. The top row indicates an ideal sensor, and the second row reports characteristics of a nonimaging hyperspectral spectrometer used commonly in ocean color research. (min. - minimum; req. required.) . . . . . . . . . . . . . . . . . . . . 74

2.2 Vendors, data, and function of the instruments deployed in the field assessment at the MVCO ASIT (Fig. 2-3). Here $\beta$ is the backscattering coefficient at a specific backscattering angle and wavelength. . . . . . . . . . . . . . . 83 
2.3 Minimum median MAPE in estimates of [chl] (top rows) and b bp650 (bottom rows), derived from radiometer-measured and imager-measured spectral radiances, comparing three glint correction approaches (polarizer, SOSC, and MASC) and the non glint corrected (uncorrected) data. The radiometer's view angle (relative to nadir) was $45^{\circ}$ and the imagers was the optimal view angle that minimized MAPE. . . . . . . . . . . . . . . . . . . 96

3.1 Ten key camera parameters (left column) and their potential impact in six conceivable drone-based applications for aquatic systems (middle columns). Greyscale shading and the number of checkmarks indicate parameters that are relatively more critical for a given remote sensing scenario. Bold checkmarks indicate specific camera parameters examined in this paper through use of the described simulation framework, with corresponding sections noted. For each camera parameter, the primary design trade-offs are noted (right column) for increases in each camera parameter. Acronyms: harmful algal bloom (HAB), chlorophyll (chl), suspended particulate matter (SPM), marginal ice zone (MIZ), phytoplankton functional type identification (PFT ID), full-well capacity (full-well cap), radiometric resolution ( $\mathrm{rad}$ res), near-infrared efficiency (NIR eff), spatial resolution (spatial res), visible (vis), price (\$), exposure time (ET), requires (req), primary production (PP), and dynamic range

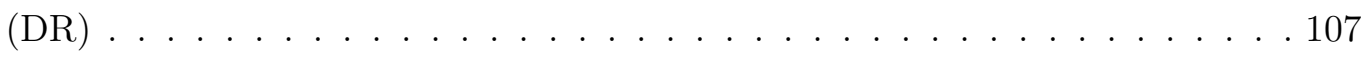

3.2 Hydrolight Simulated OAC Concentration ranges. The 'Case II' entry simulates a Case II region, where light absorption cannot be ascribed primarily to chlorophyll-containing organisms. 'Antarctic bloom' indicates a dataset simulating chlorophyll concentrations spanning the range observed during a 2001-2002 phytoplankton bloom in the West Antarctic Peninsula |Massom et al. $[2006 \mid$. In the 'Antarctic bloom' dataset, CDOM varies proportionally with the chlorophyll concentration $(\propto \mathrm{chl}) . \quad \ldots \ldots . \ldots 112$ 
4.1 Pearson's correlation coefficient (r) calculated between the 'truth' values and the predicted spectral data for each subset from the SVRs trained with spectral shift (SS), the SVRs trained without spectral shift (NSS), and re-scaled spline interpolated camera measurements (RS). Clearly the SS SVRs achieve very high correlation with the testing sets for the original dataset, data with simulated residual glint (Glint), data with a reduced signal-to-noise ratio of 10 (SNR10). This benefit even holds when just using the right half of the images (Half Image), even with added residual glint and an SNR of 10 (Half Image + Glint + SNR10). Some of the accuracy benefit holds for the laboratory generated band-pass/band-stop data, but the benefit does not hold for laboratory generated ocean color data or real world filter data. . . . . . . . . 151

4.2 The median of the MAPD calculated for each of the 53 output spectra from the SVRs trained with spectral shift (SS), the SVRs without spectral shift (NSS), and the re-scaled spline interpolated camera measurements (RS), calculated on the different data subsets (topmost column labels). Clearly the SS SVRs predicted spectra achieved much lower error on the testing dataset, but these accuracy benefits do not hold for the laboratory ocean color, bandpass/band-stop (BP/BS) data, or real-world filter measurements (right three columns). . . . . . . . . . . . . . . . . . . . . . 152

$5.1 \quad$ Example sub-meter ocean color remote sensing applications (first column) that leverage a range of deployment platforms to meet specific scientific and societal needs (second column). The chapters of the dissertation (right three columns) that benefit each application are denoted with check marks. . . . . . 166 


\section{Chapter 1}

\section{Introduction}

The ocean's color is determined by the composition and concentration of optical constituents in the water column JJerlov, 1976, Morel and Prieur, 1977]. Principal examples of optical constituents are suspended plant matter (algae), the calcium carbonate shells formed by some of the algae, and decaying organic matter. Algae, their calcium carbonate shells, and decaying organic matter each serve important roles in the ocean's biogeochemical cycles: algae are the dominant primary producers, calcium carbonate shells sequester carbon to the ocean floor, and decaying organic matter stores carbon, nitrogen, and phosphorus within the water column. Since the optical constituents determine the ocean's color, measurements of the ocean's color can be inverted, using spectral algorithms, to estimate the concentration of these key biogeochemical indicators. This process of measuring the ocean's color to characterize the ocean is known as ocean color remote sensing. Ocean waters can be categorized into two domains for ocean color remote sensing, waters where the optical constituents covary (Case I) and waters where the optical constituents vary independently (Case II) Morel and Prieur, 1977]. Simple algorithms can be used to retrieve constituent concentrations in Case I waters, but more complex algorithms that use additional spectral bands are required for retrieving constituent concentrations from Case II waters. In modern oceanography, ocean color remote sensing is primarily accomplished from satellite platforms.

In 1978, the National Aeronautics and Space Administration (NASA) deployed the Coastal Zone Color Scanner (CZCS) on-board the Nimbus 7 satellite, making it the first space-based ocean color sensor. CZCS was a proof-of-concept sensor deployed to determine if suspended plant matter could be quantified from measurement of the ocean's color from space, using the optics and electronics available at the time. CZCS measured reflected sun- 
light at $1 \mathrm{~km}$ resolution in 6 bands, four of which were for ocean color $(443 \mathrm{~nm}, 520 \mathrm{~nm}, 550$ $\mathrm{nm}$, and $670 \mathrm{~nm})$, one for terrestrial masking $(750 \mathrm{~nm})$, and one for ocean surface temperature measurement $(11,500 \mathrm{~nm})$ Austin . Researchers were able to estimate algal pigment concentrations using spectral ratios of the blue and green light $(443 \mathrm{~nm}, 520 \mathrm{~nm}$, and 550 $\mathrm{nm}$ ), but only after estimating light scattered by the atmosphere using the red band (670 $\mathrm{nm}$ ) and subtracting it from the total measured signal Gordon et al., 1980, Gordon and Morel, 1983]. The algal pigment concentration maps were then used to confirm preexisting hypotheses about the global distribution of chlorophyll and its seasonal variability Yentsch. The success of this proof-of-concept mission to recover global algal concentrations revolutionized the field of oceanography, as algae are the base of the ocean's food web and an indicator for the health of marine systems.

CZCS collected algal products for 7 years longer than intended. After the resounding success of the first ocean color satellite, various space agencies deployed next generation ocean color satellites that dramatically improved our understanding of the ocean. While this paradigm has been successful for kilometer scale sensing from high altitude platforms, the satellite ocean color remote sensing paradigm has not yet been adapted to sub-meter ocean color remote sensing from low-altitude platforms. Accurately collecting data at submeter scales is necessary for studying fine-scale physical dynamics, such as turbulent mixing, and their effects upon oceanic biology (e.g., algae). The same concepts required for satellite remote sensing can, in principle, be applied to ocean color remote sensing on sub-meter scales, but new challenges arise (Table 1.1) from the higher noise and limited spectral resolution of the available optical instrumentation, as well as changes in the characteristics of the surface reflected light (glint). This thesis focuses on the optical considerations necessary to adapt key steps of the satellite ocean color remote sensing paradigm to capture the spatially heterogeneous ocean color signal on sub-meter scales, despite capillary wave induced surface reflected light fluctuations, high dynamic range requirements, and high electronic noise.

\subsection{Evolution of the Satellite Ocean Color Remote Sensing Paradigm}

After the algal pigment concentration maps produced by CZCS were proven to match with surface measured values Gordon et al. 1980, the maps could then be used for research, 


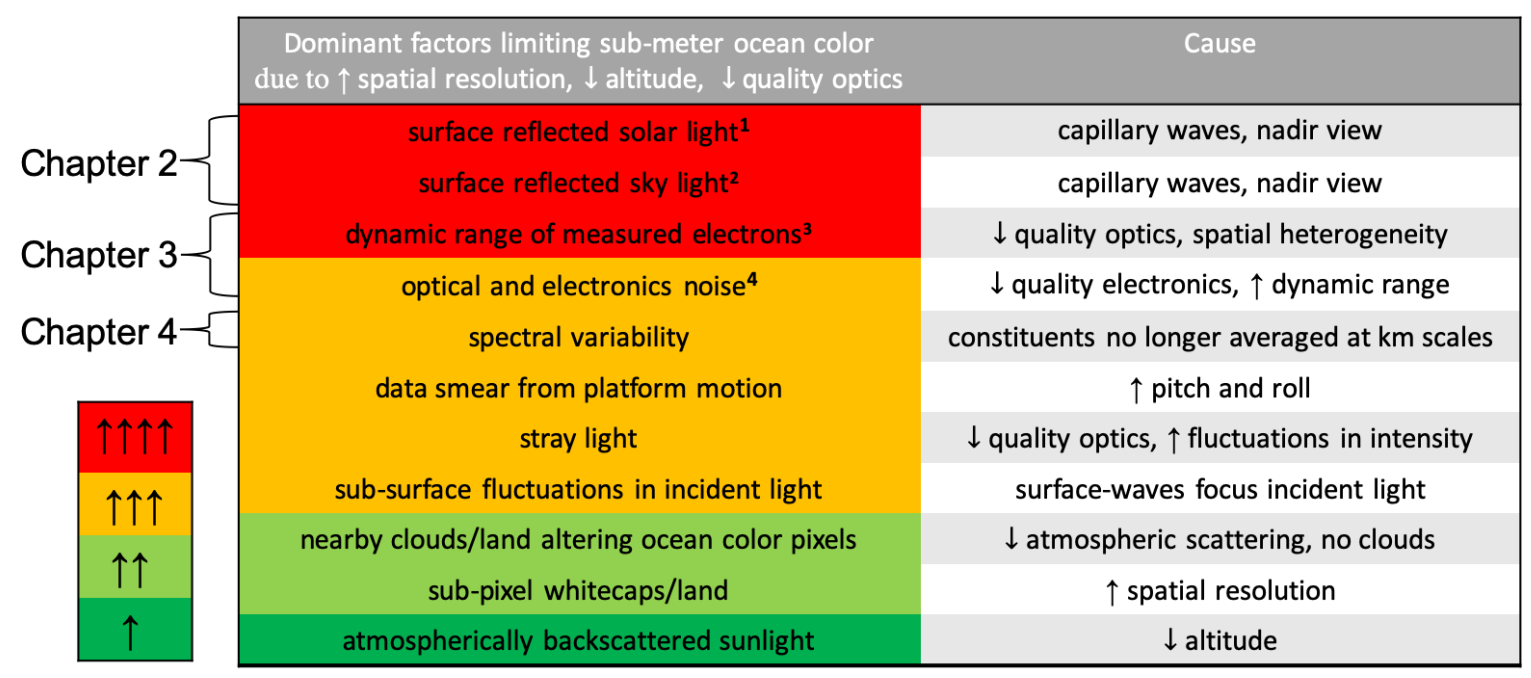

Table 1.1: The dominant factors that limit sub-meter ocean color remote sensing, where measurements are made from low altitudes with low quality optics, are dramatically different from those at $\mathrm{km}$ spatial resolutions from high altitudes with state-of-the-art optics $(1,2[\mathrm{Su}$, 2017; Larson et al., 2018; Totsuka et al., 2019; Kwon et al., 2020; Kislik et al., 2018], ${ }^{3,4}$ Kislik et al. 2018; O'Shea et al. 2020; O'Shea and Laney, 2020], ${ }^{4}$ (Honkavaara et al., 2013)). This dissertation ameliorates the highest impact factors within chapters 2-4.

either directly for studying biological processes or as indicators for physical processes. The initial maps visually confirmed preexisting hypothesis in biological oceanography, such as nutrient and light limiting the growth of algae on global scales, and the major role of ocean currents in determining algal production Yentsch. In addition to confirming prior hypotheses, the novel perspective also allowed researchers to contradict prior assumptions. In one specific case researchers determined that subsurface Gulf Stream waters had a much higher impact on the biological productivity in continental shelf waters than previously thought McClain; Ishizaka, 1990. The synoptic view provided by CZCS also alerted researchers about the extent and frequency of blooms of coccolithophore (algae that form calcium carbonate shells), which was particularly surprising as the blooms occurred in relatively well studied regions of the ocean Holligan et al. 1983. Additionally, the new perspective provided by CZCS imagery identified new bio-physical interactions. In one biological application attempting to understand the variability of algae in nearshore regions, topography was identified to play a major role in along shelf algal variability by forming saline eddies that promoted algal growth on 32-124 km scales [Yoder et al., 1987]. Finally, the algal estimates also served as indicators for physical processes, and enabled the study of oceanic features, such as warm core rings Gordon and Morel, 1983, Brown et al. 1985. Although CZCS 
was mainly intended as a proof-of-concept sensor, it ended up providing a synoptic view of bio-physical oceanographic processes in Case I waters that confirmed, refuted, and generated scientific hypotheses; all while measuring only 6 total bands of light.

After the success of CZCS, a wide variety of more advanced ocean color sensors, which provided on-orbit calibration approaches, additional spectral bands, and increased spatial resolution, were launched into space by several space agencies. The second generation sensors were: the Ocean Color and Temperature Sensor (OCTS) and the Global Imager (GLI) launched by the Japanese Aerospace Exploration Agency (JAXA) in 1996 and 2002, the Medium Resolution Imaging Spectrometer (MERIS) launched by the European Space Agency (ESA) in 2002, and the Sea-Viewing Wide Field-of-View Sensor (SeaWiFS) and the two Moderate Resolution Imaging Spectroradiometers (MODIS) deployed by NASA in 1996, 2000 and 2002. OCTS and GLI both collected less than a years worth of data, while SeaWiFS, MODIS, and MERIS (the focus of the following sections) all remained functional for at least a decade.

One of the major limitations of CZCS for long-term (decadal) studies was the lack of on-orbit calibration techniques available to compensate for decreased electronics sensitivity over time. To deal with this issue, SeaWiFS provided on-orbit calibration by monitoring the reflectance of the moon, a standard target which would not change its reflectance characteristics over a decade [McClain, 2009]. In addition to monitoring the moon, MERIS and MODIS used on-board solar diffusers to monitor the output of the sun, which served as another stable source [Barnes et al. 2004]. The increased long-term stability provided by these, and other, advanced on-orbit calibration techniques compensated for the shifts in electronic and optical efficiency on decadal scales. The new stability allowed for high impact long-term studies, including the identification the effects of El Nino Arrigo and Van Dijken, 2004 and the potential effects of global warming on marine primary production [Doney, 2006; Behrenfeld et al., 2006].

The additional spectral bands and increased spatial resolution of the second generation ocean color sensors enabled sensing in optically complex and spatially variable regions, such as the coastal ocean. Despite the name Coastal Zone Color Scanner, CZCS was mainly successful at observing features within open ocean (Case I) waters, as it lacked the spectral bands required to accurately remove atmospheric effects and compute individual optical constituent products in optically complex (Case II) regions. SeaWiFS and MODIS provided 
additional spectral bands in the near-infrared (NIR), encompassing 750 and $850 \mathrm{~nm}$, which were insensitive to variations in the optical constituents in turbid regions, and could therefore be used to correct for path radiance in these regions Gordon and Wang, 1994. The additional spectral bands in the far-red provided on MODIS and MERIS, at $665 \mathrm{~nm}, 680$ $\mathrm{nm}$, and $705 \mathrm{~nm}$ (on MERIS), enabled the retrieval of algal products in Case II regions by measuring their fluorescence Mishra and Mishra, 2012, Gurlin et al., 2011. The fluorescence in the red is due to light that algal pigments re-emit during photosynthesis, which makes the signal insensitive to fluctuations in other optical constituents in Case II regions. The additional far-blue band (at $410 \mathrm{~nm}$ ) included on the second generation ocean color satellites enabled accurate colored dissolved organic matter (CDOM, which is optically active decaying organic matter) products in Case II regions, due to CDOM's uniquely high absorption in this region of the spectrum Gordon and Wang, 1994. While the additional spectral bands increased the accuracy of individual optical constituent products, the increased spatial resolution reduced aliasing in coastal regions, which have scales of variability at or below 200 meters Moses et al. 2016. In one application, MERIS's $300 \mathrm{~m}$ resolution was sufficient to resolve gradients in the dissolved organic carbon (DOC) and colored dissolved organic matter $(\mathrm{CDOM})$ in coastal regions containing tributaries, which one kilometer resolution data (e.g., from CZCS or MODIS's ocean color bands) would not capture [Cao et al., 2018]. The increased spatial and spectral resolutions heavily advanced the range of applications that ocean color sensors could accomplish in Case II regions.

\subsection{The Current Satellite Ocean Color Remote Sensing Paradigm}

The satellite ocean color remote sensing paradigm has been developed by multiple research teams and space agencies (e.g., ESA, JAXA, and NASA) over the past 40 years of ocean color measurement McClain, 2009; International Ocean-Colour Coordinating Group, 2012a. Ocean color remote sensing is performed passively by measuring reflected sunlight. Most ocean color satellites are deployed in sun-synchronous polar orbits, which are orbits that result in satellites being over the same latitude on the earth at the same solar time each day. The sun-synchronous orbit can therefore keep the satellite (and the target below) in constant sunlight, a requirement for passive remote sensing. Ocean color sensors on polar orbiting satellites image wide swaths of the earth from high altitudes to achieve their global 


\begin{tabular}{|c|c|c|c|c|c|c|}
\hline $\begin{array}{l}\text { Ocean Color } \\
\text { Sensor }\end{array}$ & $\begin{array}{l}\text { Ocean Color Bands } \\
\text { (nm) }\end{array}$ & $\begin{array}{l}\text { Scanner } \\
\text { Type }\end{array}$ & $\begin{array}{l}\text { Swath } \\
\text { Width }\end{array}$ & $\begin{array}{l}\text { Ocean Color Band } \\
\text { Spatial Resolution }\end{array}$ & $\begin{array}{l}\text { Revisit } \\
\text { Time }\end{array}$ & $\begin{array}{l}\text { Orbit } \\
\text { Altitude }\end{array}$ \\
\hline CZCS & $443,520,550,670,750$ & Whiskbroom & $1,566 \mathrm{~km}$ & $1 \mathrm{~km}$ & 6 days & $955 \mathrm{~km}$ \\
\hline SeaWiFS & $\begin{array}{c}412,443,490,510 \\
555,670,765,865\end{array}$ & Whiskbroom & $\begin{array}{c}2,801 \mathrm{~km} \text { or } \\
1,502 \mathrm{~km}\end{array}$ & $1.1 \mathrm{~km}$ & 1 day & $705 \mathrm{~km}$ \\
\hline MODIS-Aqua & $\begin{array}{c}412,443,488,530,550 \\
665,680,748,870\end{array}$ & Whiskbroom & $2,330 \mathrm{~km}$ & $1.0 \mathrm{~km}$ & 2 days & $705 \mathrm{~km}$ \\
\hline MERIS & $\begin{array}{c}412,443,490,510 \\
560,620,665,681 \\
709,754,780,865,885\end{array}$ & Pushbroom & $1,150 \mathrm{~km}$ & $0.3 \mathrm{~km} / 1.2 \mathrm{~km}$ & 3 days & $790 \mathrm{~km}$ \\
\hline HICO & $\begin{array}{c}380-1,000 \mathrm{~nm} \\
\text { (at } 6 \mathrm{~nm} \text { resolution) }\end{array}$ & Pushbroom & $50 \mathrm{~km}$ & $0.1 \mathrm{~km}$ & Variable & $410 \mathrm{~km}$ \\
\hline
\end{tabular}

Table 1.2: The available bands and achievable spatial resolutions of the most commonly used satellite-borne ocean color sensors have slowly increased over time.

coverage. Polar orbiting satellites (CZCS, SeaWiFS, MERIS, MODIS) are deployed at very similar altitudes, but their swath widths and revisit times can vary substantially (Table 1.2. . Ocean color sensors typically view near to nadir in order to minimize atmospheric path radiance, but they often sense at $+/-20$ degrees from nadir to avoid direct solar glint. Polar orbiting satellites are able to achieve global monitoring of biogeochemical cycles in the ocean with their given viewing geometry and orbital characteristics.

The satellite ocean color remote sensing paradigm leverages knowledge and advances made within a wide range of optical disciplines to passively retrieve optical constituent products from ocean color sensors on satellites (Figure 1-1). In the first step (Figure 1-1, 1) of the passive remote sensing paradigm, sunlight propagates from the top of the atmosphere to the surface of the ocean. Along this path, the incident light is scattered by molecules and aerosols in the atmosphere into the optics of the ocean color sensor (Fig. 1-1, 2). After propagation through the atmosphere, part of the incident light (2\% at typical view-angles) reflects off the surface of the ocean and into the camera optics, while the rest of the light transmits through the ocean's surface (Fig. 1-1, 3). Most of the incident light is absorbed by water and the optical constituents within the water column, but a small percentage of the light $(<5 \%)$ is scattered by the optical constituents back up towards the surface of the ocean, and ultimately the optics of the ocean color sensor (Fig. 1-1, 4). A spectrally dispersive element in the ocean color sensor then splits the total measured signal into its color components before measurement by the camera's electronics (Fig. 1-1, 5-8). Spectral algorithms can then be applied to the measurements to estimate and then subtract the 
scattered atmospheric light, as well as the light reflected off of the surface of the ocean, to retrieve the ocean color signal (Fig. 1-1, 9). Finally, ocean color algorithms, which relate the changes in the ocean's color to the concentration of the optical constituent, are applied to the ocean color signal to calculate products such as optical constituent concentrations (Fig. 1-1, 10). The following subsections detail the complexities within each step. 

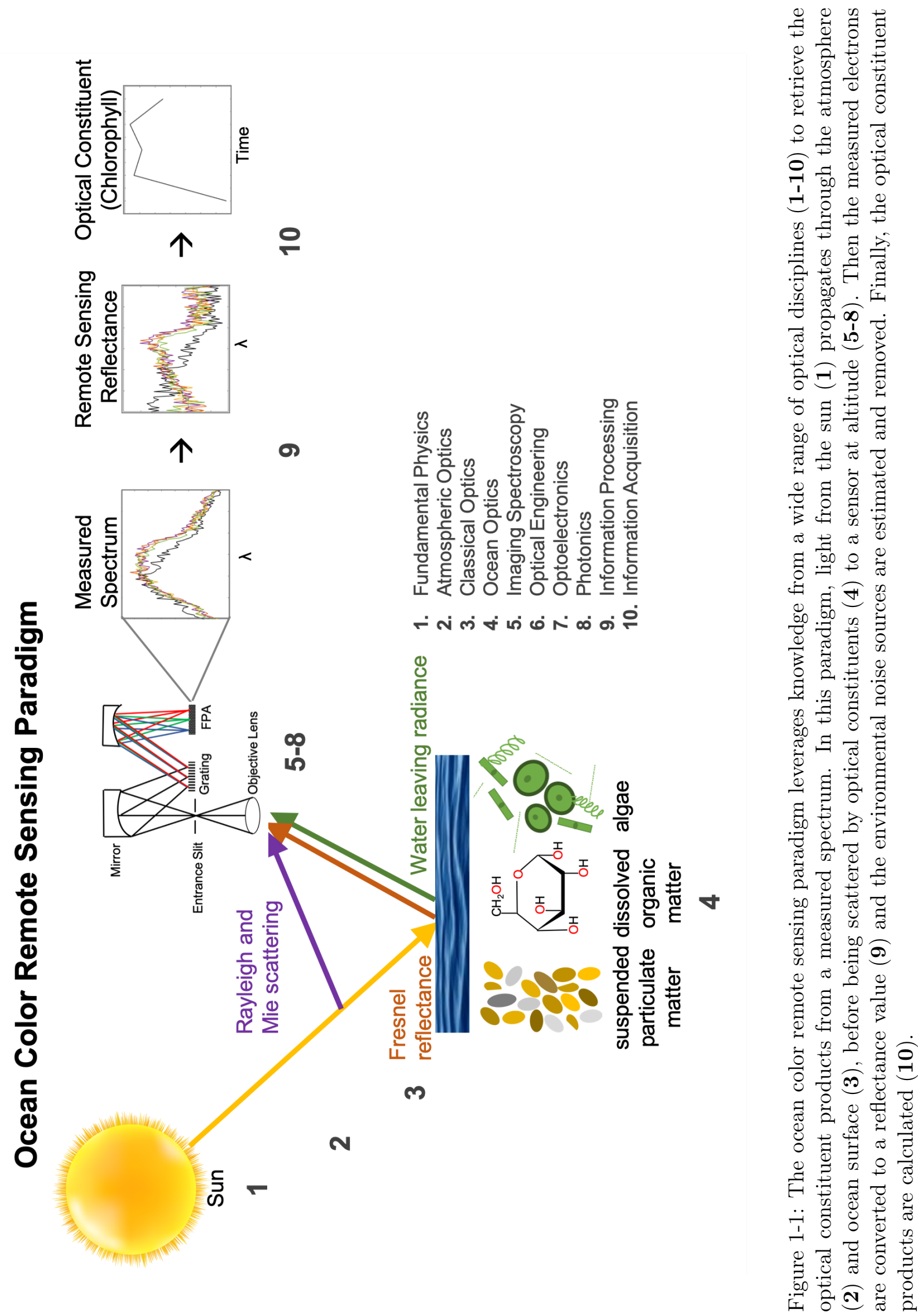


\subsubsection{Insolation of the Ocean's Surface: Fundamental Physics \& Atmospheric Optics}

Passive sensing of ocean color starts with insolation of the ocean's surface by the sun. Therefore, the available spectrum is dictated by the incident solar spectrum, which can be approximated by the radiation emitted from a $5800 \mathrm{~K}$ blackbody. The emitted radiation is low in the UV, peaks in the visible, and slowly drops off in intensity in the NIR and shortwave infrared (SWIR). To give a sense scale of the brighter part of this solar spectrum, the downwelling irradiance at the top of the atmosphere is about $1.5 \mathrm{~W} \cdot \mathrm{m}^{-2} \cdot \mathrm{nm}^{-1}$ in the visible part of the spectrum [Mobley, 2020]. The brighter visible and NIR sections are typically used for sensitive ocean color applications, however the range of usable wavelengths is further limited by interactions in the atmosphere and water column.

As the solar radiance propagates from the top of the atmosphere down to the ocean's surface it interacts with molecules and aerosols in the atmosphere. Water and oxygen molecules in the atmosphere absorb the solar radiance in sections of the red and NIR, creating variable depth features in the remote sensing reflectance data. Nitrogen dioxide $\left(\mathrm{NO}_{2}\right)$ and ozone $\left(\mathrm{O}_{3}\right)$ in the atmosphere absorb broadly across the visible, and must be corrected in the measured top of atmosphere radiance [Mobley et al. 2016c]. Some of the solar radiance is also scattered via Mie and Rayleigh scattering, depending on the size of the particles relative to the wavelength of the incident light. Light interacting with molecules, which are much smaller than the wavelengths of the incident light, undergoes Rayleigh scattering at a magnitude inversely proportional to the wavelength to the fourth power. Light interacting with aerosols, particles which are on roughly the same size scale as the incident wavelength of light, undergoes Mie scattering. The scattering has multiple effects. First, the scattering attenuates the intensity of the sunlight that reaches the surface of the ocean, and the intensity of the ocean color signal that reaches the top of the atmosphere. Second, the wavelength dependent Rayleigh scattering by molecules in the atmosphere diffuses the light over the the entire hemisphere of the sky. The skylight illuminates the ocean, and therefore shifts the color of the irradiance measured at the ocean's surface, typically towards blue. Third, some of the scattered light (referred to as path radiance) is redirected into the optics of the ocean color sensor, and overwhelms the low intensity ocean color signal. When viewing dark ocean waters the path radiance from the $500 \mathrm{~km}$ path-length of atmosphere between the sensor and 
the ocean can comprise as much as $85 \%$ of the satellites signal Orcutt, 2012. Therefore, to approximate the ocean color signal from the total measured signal, an estimate for the path radiance must be removed from the total signal.

Unfortunately, the path radiance is not spatially and temporally invariant. While the contribution of molecular scattering to the path radiance is spatially and temporally invariant, and can be computed using look-up tables with the known viewing and solar geometries as well as the windspeed conditions Whang, 2002], the aerosol contribution is not. Changes in the aerosol size distribution, index of refraction, and optical depth substantially alter the spectrum and intensity of the aerosol contribution of the path radiance Gordon et al., 1997; Nair and Moorthy, 1997|. Depending on the region and local conditions, the aerosols could be comprised of soot, smoke, sea salt, dirt, or even ice crystals, which each have unique absorption and scattering characteristics Gordon et al., 1997; Chin et al., 2009: Mobley et al., 2016b. Overall, the aerosol imposed variations in the path radiance limit the accuracy with which the ocean's color can be retrieved from the total measured signal.

Accurately removing the atmospheric path radiance requires using a spectral algorithm that takes advantage of bands that do not co-vary with optical constituents (which determine the ocean's color). Learning from the limitations of CZCS's sole red band for atmospheric removal above turbid waters, MODIS included bands in the NIR at $748 \mathrm{~nm}$ and $869 \mathrm{~nm}$, which covary significantly less with turbidity [Mobley et al. 2016c]. To begin the atmospheric correction process, the ratio between the long and short NIR bands $[\epsilon(748,869)]$ is taken as:

$$
\epsilon(748,869)=\frac{\rho_{\mathrm{TOAC}}(748)}{\rho_{\mathrm{TOAC}}(869)}
$$

where $\rho_{\text {TOAC }}$ is the reflectance calculated from the top of atmosphere radiance that has been corrected for whitecaps, Rayleigh radiance, surface reflected skylight (included in the Rayleigh radiance correction), surface reflected sunlight, $\mathrm{O}_{3}$ attenuation, and $\mathrm{NO}_{2}$ attenuation Gordon and Wang, 1994, Mobley et al., 2016c. The relative humidity and $\epsilon(748,869)$ are then used with predefined look up tables, specified for given solar and view geometries, to identify the NIR ratios of the two aerosol types that bound $\epsilon(748,869)$ [known as $\epsilon_{\text {low }}(748,869)$, and $\epsilon_{\text {high }}(748,869)$ ] Ahmad et al., 2010]. It is then assumed that the proportional difference $(\Delta)$ in $\epsilon(748,869)$ relative to the look up table values holds across 
wavelength as:

$$
\Delta=\frac{\epsilon(748,869)-\epsilon_{\text {low }}(748,869)}{\epsilon_{\text {high }}(748,869)-\epsilon_{\text {low }}(748,869)}
$$

Therefore, the reflectance due to aerosols at any wavelength $\left[\rho_{\mathrm{A}}(\lambda)\right]$ can be calculated from:

$$
\rho_{\mathrm{A}}(\lambda)=\left[(1-\Delta) * \epsilon_{\mathrm{low}}(\lambda, 869)+\Delta \epsilon_{\mathrm{high}}(\lambda, 869)\right] * \rho_{\mathrm{TOAC}}(869) .
$$

The spectral reflectance due to aerosols can then be subtracted from the corrected reflectance to get the water-leaving reflectance. This process assumes that the water-leaving reflectance in the NIR is negligible and that there are no strongly absorbing aerosols. In cases where the water-leaving reflectance in the NIR is non-negligible, an extended process is implemented. After determining the water-leaving reflectance from the above equations, a series of equations then iteratively estimates the actual reflectance from estimates of the absorption due to chlorophyll and backscatter from particles. Finally, the actual remote sensing reflectance at the two correction wavelengths is removed from the total reflectance, before re-implementing the algorithm from the first step [Bailey et al. 2010]. In summary, the two NIR wavebands are critical for path radiance estimation and correction in both open ocean and turbid regions.

\subsubsection{Transmission and Reflection at the Ocean's Surface: Classical Optics}

After propagating through the atmosphere, incident sunlight next interacts with the ocean surface. At the ocean's surface, a fraction of the incident sunlight is reflected back into the atmosphere, and the remainder is coupled into the ocean. The Fresnel equations describe the amount of light that is transmitted, or reflected, at any given incidence angle and polarization state, for a planar surface.

$$
\begin{aligned}
& R_{\mathrm{s}}{ }^{1 / 2}=\frac{n_{1} \cos \left(\theta_{\mathrm{i}}\right)-n_{2} \cos \left(\theta_{\mathrm{t}}\right)}{n_{1} \cos \left(\theta_{\mathrm{i}}\right)+n_{2} \cos \left(\theta_{\mathrm{t}}\right)} \\
& {R_{\mathrm{p}}}^{1 / 2}=\frac{n_{1} \cos \left(\theta_{\mathrm{t}}\right)-n_{2} \cos \left(\theta_{\mathrm{i}}\right)}{n_{1} \cos \left(\theta_{\mathrm{t}}\right)+n_{2} \cos \left(\theta_{\mathrm{i}}\right)}
\end{aligned}
$$

Here, $\mathrm{n}_{1}$ is the index of refraction of the air (1.0), $\mathrm{n}_{2}$ is the index of refraction of salt water (1.34), $\theta_{\mathrm{i}}$ is the incident angle (typically $<20^{\circ}$ ), and $\theta_{\mathrm{t}}$ is the transmitted angle. The Fresnel 
equations can be simplified to only depend upon the incidence angle, $\theta_{\mathrm{i}}$ through substitution of Snell's law:

$$
n_{1} \sin \left(\theta_{\mathrm{i}}\right)=n_{2} \sin \left(\theta_{\mathrm{t}}\right)
$$

At the off-nadir view angles typical of satellite remote sensing, the reflection coefficients for both polarization states are around $4 \%$. Therefore, roughly $4 \%$ of the incident sunlight and skylight are reflected off of the surface of the ocean and into the optics of the ocean color sensor. Although this seems quite low, it is often higher than the typical reflectance of constituents in the open ocean water column $(<2 \%)$, and therefore requires avoidance (for the surface reflected sunlight) and correction (for the surface reflected skylight). Sun-glint, the more intense of the two surface reflected light sources, can be mitigated by imaging with the ocean color sensor at slightly off-nadir view angles, so the solar disk is not within the image. The sky glint is typically removed during molecular scattering correction, by assuming that the reflectance is wind-speed and view-angle dependent Wang, 2002]. The sun and sky light that is not reflected at the ocean's surface is instead transmitted through the air-sea interface, where it interacts with optical constituents in the water column.

\subsubsection{Absorption, Scattering, and Fluorescence in the Water Column: Ocean Optics}

Once transmitted through the air-ocean interface the incident solar radiation can interact, through absorption or scattering, with the optical constituents within the water column. The radiative transfer of light through the ocean is characterized by the water column's inherent optical properties (IOPs), properties that are due to the optical constituents and are invariant to changes in the light field [Preisendorfer, 1976]. The absorption coefficient, scattering coefficient, and the volume scattering function are the principal IOPs that dominate optical propagation through the water column. While the IOPs describe the radiative transfer within the water column, they cannot be measured directly via remote sensing. Instead apparent optical properties (AOPs), properties that vary with sensing conditions and viewing geometries (e.g., the water-leaving radiance), are used to estimate both the constituent concentrations and the IOPs.

Light in the water column decreases exponentially with depth, due to absorption and scattering by both water and the optical constituents. The depth above which $90 \%$ of 
the remotely sensed upwelling irradiance originates is referred to as the penetration depth Gordon and McCluney, 1975]. The penetration depth varies heavily based on the optical constituents. In the clearest unproductive, oligotrophic, ocean regions such as the north pacific gyre, the penetration depth of blue light is approximately 50 meters, while the penetration depth of red light is around 2 meters. In typical Case I open ocean waters, such as the center of the north pacific gyre, where the optical constituents covary with suspended plant matter, the penetration depth of blue light is around 25 meters and the penetration depth of red light is slightly less than 2 meters. In the most turbid Case II coastal waters, such as the Baltic Sea, the penetration depth of both blue and red light can be reduced to less than 1 meter. Therefore, products calculated from different spectral bands or in different regions describe the optical constituents at different depths.

The IOPs are clearly important to recover via remote sensing, as they describe optical propagation in the water column, but the concentrations of the optical constituents, such as suspended plant matter, colored dissolved organic matter, and suspended particulate matter, are also important as they serve as indicators of key biogeochemical cycles (e.g., the carbon cycle) in the ocean. Nearly half of the Earth's primary production, where organic compounds are produced within organisms from inorganic compounds, occurs in the ocean. Thus, suspended plant matter, the dominant primary producer in the ocean, is a key part of the oceanic carbon cycle and the base of the food chain in much of the ocean Falkowski and Raven, 2007; Malone et al. 2016. Not only does suspended plant matter contribute to organic carbon cycling, but also inorganic cycling. Suspended plant matter that forms calcite shells, known as coccolithophores, contribute $75 \%$ of the ocean originating inorganic carbon sequestered to the ocean floor Gordon and Balch, 1999]. Another constituent, colored dissolved organic matter (CDOM), serves as a tracer for total dissolved organic matter (DOM). Dissolved organic matter is important to oceanic health because it releases nitrogen, inorganic carbon, and trace metals into the water column when it is destroyed by photobleaching, and these elements are used during primary production Coble, 2007]. A final constituent of import, suspended particulate matter, can be comprised of dirt, silt, sand, or detritus (decaying organisms). Suspended particulate matter impacts oceanic health by adding to nutrient availability for primary production and limiting light availability. Clearly, the optical constituents in the ocean can serve as important indicators of the biogeochemical cycles, as well as marine health. 
Seawater and the optical constituents within the water column each have their own typical absorption and scattering properties due to their physical characteristics. Water itself absorbs quite strongly in the far red and near-infrared (NIR), weakly in the visible, and moderately in the ultraviolet Dorsey, 1940; Pope and Fry, 1997]. The molecular structure of water $\left(\mathrm{H}_{2} \mathrm{O}\right)$ causes local absorption maxima within water's absorption spectrum at multiple frequencies above $450 \mathrm{~nm}$, due to the harmonics in the $\mathrm{O}-\mathrm{H}$ bond vibrational mode. Water's polarity allows it to form clusters of hydrogen bonded molecules which change its absorption characteristics, however temperature and salinity can affect the formation of these clusters. Increases in temperature can break apart the weak hydrogen bonds, forcing smaller clusters, while increases in salinity can cause the hydrogen bonds to form larger clusters. The temperature and salinity effects must be calibrated when using the absorption of water as a constant, especially in the NIR [Pegau et al., 1997].

Suspended plant matter (algae) are primary producers within the ocean that have evolved to convert sunlight into chemically stored energy, in a process known as photosynthesis. The dominant pigment that absorbs sunlight in algae, chlorophyll a, absorbs heavily in the blue and red Clarke et al., 1970, Morel and Prieur, 1977. While pigments, and therefore the spectral shape, vary from species to species, there is a general tendency for the pigments to absorb in the blue and red Bricaud et al. 2004. Although most of the absorbed light is either used during photosynthesis or turns into heat, some of the absorbed light excites molecules that emit light at longer wavelengths when they return to non-excited states (i.e., they fluorescence). While chlorophyll fluorescence has a relatively minor intensity, its presence at this particular wavelength is unique, which makes it useful for estimating chlorophyll in optically complex (Case II) waters Abbott and Letelier. Overall, due to the high absorption in the blue and red, and scattering in the green, waters with high concentrations of suspended plant matter are characteristically green.

The dissolved component within the water column, operationally defined as being able to pass through a filter with pore sizes ranging from 0.2-0.7 um, is typically comprised of humic substances originating from soil runoff in coastal regions and fulvic acids originating from phytoplankton in open ocean regions Mostofa et al., 2009]. While not all dissolved organic matter substantially affects the ocean's color, the part that does (CDOM) has a logarithmically decreasing absorption curve, which dominates in the ultraviolet and blue Morel and Prieur, 1977; Gholizadeh et al. 2016. The wide variety of dissolved materials 
results in an exponentially decreasing absorption spectrum $\left[a_{\mathrm{CDOM}}(\lambda)\right]$ with few features Coble, 2007, which can be represented by the following equation Bricaud et al., 1981:

$$
a_{\mathrm{CDOM}}(\lambda)=a_{\mathrm{CDOM}}\left(\lambda_{0}\right) \exp \left[-S\left(\lambda-\lambda_{0}\right)\right],
$$

where $\mathrm{S}$ is the mean (or spectral) slope, $\lambda_{0}$ is a reference wavelength (typically in the blue), and $\lambda$ is the desired wavelength. The spectral slope can be indicative of the origin (terrestrial or marine) of the CDOM. Equation 1.7 can be used to calculate the absorption due to CDOM at higher wavelengths and therefore correct for CDOM absorption effects across the spectrum. Scattering by CDOM is typically assumed to be negligible. CDOM can also fluoresce, though the exact wavelength can vary depending upon the chemical composition of the dissolved organic matter.

Suspended particulate matter (SPM), operationally defined as being unable to pass through a filter of 0.2-0.7 um pore size, is typically comprised of suspended sand, sediment, dirt, or the calcium carbonate shells of coccolithophores. The relatively large size of SPM, relative to the visible and NIR wavelengths of light, leads to a wavelength independence in the scattering of light [Kirk, 1994]. The scattering is most notable in the red and NIR sections of the water-leaving radiance section, where water absorption typically dominates.

\subsubsection{Measuring Ocean Color: Spectral Imaging}

After propagating through the air-sea interface, and back up through the atmosphere, the incident light is then imaged into its component colors by a spectral camera on-board the satellite platform. A myriad of spectral separation techniques exist for multispectral and hyperspectral cameras, but only a few are optimal for ocean color imaging. Fabry-Pérot Interferometers, wedge filters, linear variable filters, and rotating multispectral filter wheels are unable to simultaneously image the spectral content of a single spatial location, so are not

typically used by ocean color sensors. Since satellites typically move at $7.5 \mathrm{~km} \cdot \mathrm{s}^{-1}$ McClain et al. 2014], the spectral bands derived from these approaches would image slightly different spatial regions, which is undesirable for ocean color product retrieval as the estimation algorithms leverage ratios between the bands. Bandpass filters and grating are the spectral separation techniques typically used in ocean color remote sensing, as they are able to 
simultaneously image the spectral bands of a given spatial location. Most ocean color sensors (CZCS, MERIS, Hyperion, HICO, OLCI) leverage a dispersive grating to separate the spectra, though a couple of the earlier sensors (MODIS and SeaWiFs) used bandpass filters Qian, 2020, McClain et al. 2014.

Historically, each ocean color sensor utilized a unique spectral imaging system. CZCS was a whiskbroom, or scanning, spectral imager. It used a rotating mirror to spatially scan perpendicularly to the satellites orbit track McClain et al., 2014. The scanning mirror had the ability to tilt to avoid sun-glint. Spectral dispersion was achieved by using a grating, and sampling the spectrally separated light at the appropriate spatial locations. MODIS was also a whiskbroom sensor that used a rotating mirror. Unlike CZCS, spectral separation was not accomplished via a grating, but instead via dichroic beamsplitters and filters on the focal plane arrays. The system used four focal plane arrays to capture the wide intensity range of the incident radiation. MERIS was a pushbroom sensor, which is a sensor that simultaneously acquires spectra from a spatial line perpendicular to the satellites orbital trajectory. Since the sensor simultaneously acquired spatial and spectral data, it could utilize higher exposure times for each spatial region, as it did not have to spatially scan. MERIS spectrally dispersed light by using gratings. Unfortunately, since MERIS uses 5 different cameras for the full spectral information, there can be discontinuities in the ocean color products [McClain et al. 2014. Despite the idiosyncrasies with the data from the 5 different cameras on MERIS, image correction approaches made the data useful for ocean color remote sensing.

\subsubsection{Signal Detection: Photonics \& Optoelectronics}

After being split into the component spectra, the light must be imaged and converted into digital numbers for digital recording. Photons from the ocean's surface are imaged onto the focal plane array and are converted to electrons at the spectral quantum efficiency of the focal plane array (imaging sensor). The integration time of the imager controls the amount of time that photons are converted into electrons. The number of electrons captured by the detector is a function of surface area being imaged, the solid angle of the aperture, the input radiance, the optical efficiency, and the spectral bandwidth:

$$
N_{\mathrm{E}}=L_{\mathrm{t}} \cdot \Omega_{\text {aperture }} \cdot A_{\text {surface }} \cdot t \cdot E_{\text {Optical }} \cdot E_{\text {Quantum }} \cdot \Delta \lambda \cdot \frac{\lambda}{h c} .
$$




\begin{tabular}{|cccc|}
\hline $\begin{array}{c}\text { Ocean Color } \\
\text { Sensor }\end{array}$ & $\begin{array}{c}\text { Full-well } \\
\text { Depth }(e)\end{array}$ & SNR & $\begin{array}{c}\text { Radiometric Resolution } \\
\text { (bits) }\end{array}$ \\
\hline CZCS & $3 * 10^{4}$ & $100-150$ & 8 \\
SeaWiFS & $\left(2 \times 10^{6}\right)$ & $800-1,400$ & 10 \\
MODIS-Aqua & $\left(1 \times 10^{6}\right)$ & $500-1,000$ & 12 \\
MERIS & $6 \times 10^{5} / 2.4 \times 10^{6}$ & $200-1,180$ & 12 \\
HICO & $2 \times 10^{5} / 8 \times 10^{5}$ & 200 & 14 \\
\hline
\end{tabular}

Table 1.3: The increasing radiometric resolution and SNR demonstrate the advancement of the ocean color imager electronics through the ocean color sensor deployments. The lower SNR of HICO is due to the increased spectral resolution. Full-well depths in parenthesis are estimated based on the maximum stated SNR Qian, 2015, Lucke et al., 2011, Bezy et al., 1997; Qimaging.

where $N_{\mathrm{E}}$ is the number of electrons on the imager, $L_{\mathrm{t}}$ is the total radiance at the sensor, $\Omega_{\text {aperture }}$ is the solid angle of the instruments aperture, $A_{\text {surface }}$ is the area of the surface being measured, $t$ is the exposure time, $E_{\text {Optical }}$ is the optical efficiency, $E_{\text {Quantum }}$ is the quantum efficiency of the focal plane array, $\Delta \lambda$ is the spectral bandwidth, $\lambda$ is the wavelength, $h$ is Planck's constant, and $c$ is the speed of light Mobley et al. 2016a. Clearly increasing the spatial resolution (decreasing $A_{\text {surface }}$ and $t$ ) or increasing spectral resolution (decreasing $\Delta \lambda)$ limits the amount of photons captured. Therefore, increases in the spatial or spectral resolution must be offset by increasing the optical efficiency, solid angle, or exposure time to maintain the same signal-to-noise ratio (SNR). Since satellites use the state-of-the-art sensors, they typically trade spatial for spectral resolution (and vice versa) to achieve their remote sensing goals.

The imaged electrons are stored within the camera's full-well, and cannot exceed the fullwell capacity of the imager. The full-well capacity is typically around one million electrons on second generation ocean color sensors (Table 1.3). The number of electrons in the fullwell is converted to a digital number via the analog-to-digital converter (ADC), which has a specific radiometric resolution as measured in bits (typically 10-14 bits for second generation ocean color sensors), for digital storage Qian, 2020, McClain et al., 2014. The wide dynamic range (from the full-well capacity of one million electron) and high radiometric resolution (from the 10-14 bit ADCs) are necessary because of the large dynamic range between the dark ocean color pixels and the bright land, ice, and cloud pixels typical of oceanic images.

In addition to the previously discussed environmental noise sources, optical and electronic 
noise sources further limit the accuracy of the measured total radiance. Since satellite sensors have large full-well capacities, signal dependent shot noise typically dominates. Shot noise occurs due to the discretization of photons, and follows a Poisson distribution McClain et al. 2014. The next highest noise source is typically the quantization noise. The quantization noise results from round-off error from when the ADC converts electrons in the full well to a digital number [Boncelet, 2005], and follows a uniform distribution. Other common noise sources, which are typically minimal for satellite sensors, are dark noise, which is temperature and time dependent Gow et al., 2007; Farrell et al., 2012], and read noise, which is a result of the electronic readout within the camera system [Chen et al., 2009]. Since spatially scanning hyperspectral imagers measuring the ocean color signal image a wide dynamic range, it is possible for shot-noise to dominate in one region of the spectrum while another noise source, such as quantization noise, dominates in another section of the spectrum.

Both dark noise and the temperature dependence of the electronics must be corrected for when used for sensitive ocean color applications. MODIS included a space view port, through which it could image dark sections of the sky, to estimate the dark noise in the images Xiong et al. 2004. The estimate of the dark noise could then be subtracted from the images of the Earth to remove dark noise effects. Another optoelectronic effect was the temperature dependence of the measured signal on the focal plane array. SeaWiFS included thermistors on-board each focal plane array to monitor the temperature Barnes and Zalewski, 2003. The correction removed temperature effects using pre-computed coefficients, inherently assuming that the temperature dependence would not change in orbit Barnes et al., 1994. Overall, the dark-noise and temperature dependent effects on the signal can be largely ameliorated with these correction techniques.

\subsubsection{Mitigating Optical Errors: Optical Engineering \& Information Processing}

A major limitation of using CZCS data for timeseries analysis was the lack of a method for long-term on-orbit stability monitoring of the optical and electronic components. The calibration process is critical, as errors as small as $1 \%$ in the satellite measured radiance can result in errors of $10 \%$ in the water-leaving radiance, because the path radiance is such a significant portion of the signal [Barnes et al., 2001; Mobley et al., 2016b]. The space 
agencies that launched the second generation of ocean color sensors included a variety of different optical components to aid in on-orbit calibration of the top of the atmosphere total measured radiance.

SeaWiFS used a four step radiometric calibration approach to accurately compare measurements made over the entire instrument deployment. The first step was a lab-based characterization, where the MODIS sensor measured an integrating sphere with a National Institute of Standards and Technology (NIST) traceable lamp. The second was the measurement of the solar disk before and after launch, using a solar diffuser, to determine changes in the optical train due to the launch [Barnes et al., 2001]. The measurement of the solar disk allows for changes in the diffuser-instrument system to be characterized after the sensor had been launched into orbit. The third step was measurement of the lunar surface, which served as a stable and independent diffuser that could be measured to characterize fluctuations in the instrument response without containing degradation of the solar diffuser. SeaWiFS pitch-axis momentum wheels rotate the sensor at specific time intervals to view the moon under similar conditions on an approximately monthly basis. After correcting for variations in the sensing conditions and oversampling of the moon due to rotation of the sensor, the moon can be used to determine long-term trends in the variations in the optics of the sensor. The solar diffuser also makes daily measurements of the solar radiation, to determine if there were discontinuities in the degradation of the sensors performance, which must be known to apply the lunar calibration. The degradation in the response of SeaWiFS band $8(865 \mathrm{~nm})$ was as much as $12 \%$ over just 3 years time, so clearly this band requires recalibration for determining trends in ocean color data Barnes et al. 2004. The fourth, and final, step is a vicarious calibration, which forces the radiometrically and atmospherically corrected top of the atmosphere measured radiance to match with in-situ radiometric measurements made in an optically homogeneous region. SeaWiFS uses data collected by the Marine Optical Buoy (MOBY) to correct in the visible, and data collected in the Pacific and southern Indian oceans to correct the NIR [Franz et al., 2007]. The on-orbit calibration methods reduce errors sufficiently to be able to compare measurements made over multiple years.

In addition to solar diffuser and lunar monitoring capabilities, MODIS also includes a Spectroradiometric Calibration Assembly (SRCA) and a Solar Diffuser Stability Monitor (SDSM) for further calibration Xiong et al., 2004. The SRCA enables on-board spectral, 
radiometric, and spatial calibration. The spectral calibration data is produced by an onboard light source that is spectrally filtered using a monochromator, before it is redirected onto MODIS's scan mirror. To collect radiometric data, the SRCA uses the on-board source with a feedback diode to control the input radiance to MODIS and repeats this process for 6 different radiance levels. The spatial registration is performed by the SRCA placing a spatial pattern, called a reticle, as the input to MODIS's scan mirror, so that it can align the various focal plane arrays. Despite having the on-board calibration source, the solar diffuser is typically used to calibrate MODIS. The SDSM iteratively monitors the sun and the solar diffuser to calibrate the radiometric correction due to degradation of the solar diffuser. Overall, the SRCA and SDSM provide further on-board calibration capabilities than MODIS's predecessors.

In addition to the standard optical aberrations and uncertainties in imaging systems (e.g., defocus, chromatic aberration, etc.), a few specific optically induced errors have been prominently featured in ocean color sensors. In MODIS, the spectral imaging technique introduced striping errors in the measured radiance. Images captured by the multi-detector bandpass filter used on MODIS suffered from alternating bright and dark lines (striping) due to differences in the detectors [Rakwatin et al., 2007]. Specific algorithms were required to correct for these bands, to accurately estimate the ocean color signal. Another source of error in MODIS was stray-light from nearby bright targets (e.g., clouds) Meister et al. 2008]. Stray light within an imaging system is the light that has been been scattered off of the internal housing into pixels capturing light from other targets. Stray light has a particularly large impact in ocean color remote sensing due to the wide dynamic range of the signals being measured. Since radiance emanating from the ocean is typically much darker than either land, clouds, or sun glint, stray light from these sources becomes a significant concern. Without correction, water pixels adjacent to these sources could be affected by stray light and have their estimated radiances significantly altered. Therefore, baffling and pre-deployment characterization of stray light correction techniques are also important considerations for ocean color remote sensing tasks.

\subsubsection{Ocean Color Algorithms: Information Acquisition}

The radiometrically corrected data, which was collected under a wide range of measurement conditions (e.g., view angles, solar zenith angles, Earth-Sun distances, etc), must be 
normalized before it can be used in ocean color algorithms Mobley et al. 2016b. The measured water-leaving radiance is typically converted to either the remote sensing reflectance $\left[R_{\mathrm{rs}}(\lambda)\right]$ or the normalized water-leaving reflectance. The remote sensing reflectance is simply the water-leaving radiance divided by the downwelling irradiance above the surface. The normalized water-leaving reflectance is similarly the water-leaving radiance divided by the downwelling irradiance, but it also attempts to mathematically correct for solar zenith angle, the earth sun distance, and atmospheric attenuation.

After the water-leaving reflectance signal has been isolated from the environmental noise, ocean color algorithms can be applied to the normalized reflectance data to recover biogeochemical information, typically referred to as ocean-color products. Basic ocean color algorithms typically leverage ratios in different bands in the visible and NIR spectrum to retrieve the optical constituent concentrations. One widely used algorithm for open ocean chlorophyll (a proxy for algae) concentration retrieval is the ocean color 4 (OC4) algorithm O’Reilly et al., 1998:

$$
\begin{array}{r}
\log _{10}([c h l])=C_{4} \log _{10}^{4}(M B R)+C_{3} \log _{10}{ }^{3}(M B R)+C_{2} \log _{10}^{2}(M B R)+ \\
C_{1} \log _{10}(M B R)+C_{0},
\end{array}
$$

where $[\mathrm{chl}]$ is the chlorophyll concentration, $\mathrm{C}_{\mathrm{i}}$ are the empirically derived coefficients, and MBR is the maximum band ratio:

$$
M B R=\left(\frac{\max \left(R_{\mathrm{rs}}(443 n m), R_{\mathrm{rs}}(490 \mathrm{~nm}), R_{\mathrm{rs}}(510 \mathrm{~nm})\right)}{R_{\mathrm{rs}}(560 \mathrm{~nm})}\right) .
$$

Here, $\mathrm{R}_{\mathrm{rs}}(\lambda)$ is the remote sensing reflectance at a specific wavelength $(\lambda)$. The OC4 algorithm leverages a band ratio between blue and green to estimate the concentration of chlorophyll in the water. The coefficients $\left(\mathrm{C}_{\mathrm{i}}\right)$ are empirically derived for each satellite instrument though the use of in-situ data that is temporally aligned up with satellite retrieved water-leaving radiances. Similar band ratio algorithms exist for estimating CDOM and SPM concentrations, which leverage their specific spectral absorption and scattering features (section 1.2.3). Hyperspectral algorithms also exist for more complex tasks such as CDOM spectral slope estimation, pigment separation [Chase et al., 2017], and differentiating between phytoplankton functional types (phytoplankton function type identification) 
Aiken et al. 2014; Lubac et al., 2008.

Semi-analytical algorithms, which combine radiative transfer theory with empirical measurements, can also be used to retrieve ocean-color products. Inherent optical properties can be estimated from remote sensing reflectance measurements by using the Generalized Inherent Optical Property (GIOP) model with region specific assumptions Werdell et al. 2013; Franz and Werdell, 2010]. The GIOP model solves for the magnitude of the absorption and scattering of different optical constituents through iteratively attempting to match the measured subsurface remote sensing reflectance to the modeled subsurface remote sensing reflectance. Following Lee et al. Lee et al., 2002, the measured subsurface remote sensing reflectance $\left(\mathrm{r}_{\mathrm{rs}}\right)$ can be calculated from the remote sensing reflectance:

$$
r_{\mathrm{rs}}(\lambda)=\frac{R_{\mathrm{rs}}(\lambda)}{0.52+1.7 * R_{\mathrm{rs}}(\lambda)} .
$$

The modeled remote sensing reflectance can be related to the absorption and backscattering, following Gordon et al. Gordon et al., 1988, as:

$$
r_{\mathrm{rs}}(\lambda)=0.0949 * \frac{b_{\mathrm{b}}(\lambda)}{a(\lambda)+b_{\mathrm{b}}(\lambda)}+0.0794 *\left(\frac{b_{\mathrm{b}}(\lambda)}{a(\lambda)+b_{\mathrm{b}}(\lambda)}\right)^{2}
$$

where $a(\lambda)$ is the total absorption, $b_{\mathrm{b}}(\lambda)$ is the total backscattering, and the coefficients are empirically determined. The total backscattering and absorption can be split into spectral components with magnitudes as:

$$
b_{\mathrm{b}}(\lambda)=b_{\mathrm{bw}}(\lambda)+M_{\mathrm{bp}} * b_{\mathrm{bp}}(\lambda)
$$

where $b_{\mathrm{bw}}(\lambda)$ is the water contribution calculated from the remotely sensed temperature and estimated salinity from prerecorded measurements, $b_{\mathrm{bp}}(\lambda)$ is the particulate backscattering spectral shape, and $\mathrm{M}_{\mathrm{bp}}$ is the magnitude of the scattering. For absorption it can be broken into magnitude and spectral shape components from water $\left[a_{\mathrm{w}}(\lambda)\right]$, phytoplankton $\left[M_{\mathrm{ph}}\right.$ and $\left.a_{\mathrm{ph}}(\lambda)\right]$, and colored dissolved organic matter $\left[M_{\mathrm{CDOM}}\right.$ and $\left.a_{\mathrm{CDOM}}(\lambda)\right]$ :

$$
a(\lambda)=a_{\mathrm{w}}(\lambda)+M_{\mathrm{ph}} * a_{\mathrm{ph}}(\lambda)+M_{\mathrm{CDOM}} * a_{\mathrm{CDOM}}(\lambda) .
$$

The absorption due to water $\left(a_{\mathrm{w}}\right)$ is known [Pope and Fry, 1997, and typically the spec- 
tral absorption for phytoplankton and $\operatorname{CDOM}\left[a_{\mathrm{ph}}(\lambda)\right.$ and $\left.a_{\mathrm{CDOM}}(\lambda)\right]$ are assumed for a particular region. Using these equations, the magnitudes $\left(M_{\mathrm{x}}\right)$ are iteratively solved for by comparing the modeled subsurface remote sensing reflectance to the measured subsurface remote sensing reflectance at as many wavelengths as there are unknowns. Therefore, the estimate of the magnitude of the absorption due to colored dissolved organic matter, phytoplankton, and particulates can be estimated simultaneously. While users can change the specific assumptions that are used, to be region or task dependent, NASA leverages specific assumptions by Maritorena et al. Maritorena et al. 2002 in their default product. Overall, through the use of either empirical or semi-analytical algorithms, a wide range of inherent and apparent oceanic properties can be remotely retrieved from spectral images.

\subsection{Challenges in Ocean Color Remote Sensing on Meter Scales: Limitations of the Satellite Paradigm}

Space-borne ocean color sensors are still unable to meet the spatio-temporal resolution combinations required to image many coastal and inland processes Mouw et al. 2015, Robinson, 2010, despite the significant advances in satellite ocean color remote sensing over the past 40 years. Coastal and inland processes can occur on sub-meter and subhourly scales in sub-km scale regions Mouw et al., 2015, Robinson, 2010; International Ocean-Colour Coordinating Group, 2012a. Physical dynamics, such as Langmuir cells and turbulent patches, can impact the local environment, and in turn alter the ocean color signal on sub-meter scales (Figure 1-2 International Ocean-Colour Coordinating Group, 2012a. Not only is the ocean color signal different on these scales, but also the noise sources. Dynamic physical processes, such as surface and capillary waves, can alter the reflections and transmission characteristics of the solarlight and skylight at sub-meter scales (Figure 1-2, yellow circle). Accurately measuring variations in the ocean color signal is critical not only to study the impact of the physical processes at this scale, but also to inform the effects of the sub-pixel variability on the coarser resolution (5-1,000 m) products provided by ocean color sensors on-board satellites and aircraft, as the coarser resolution products are not always equivalent to the mean of the finer resolution products Lee et al. 2012. Overall, polar orbiting satellites do not provide sufficient spatio-temporal resolution to study the dynamic physical processes in coastal ocean regions, but while hyperspectral cameras on 
low-altitude drone platforms can provide sufficient spatio-temporal resolution, changes in the optical characteristics of the surface at this scale will limit the accuracy of the retrieval of the ocean color data.

The spatio-temporal limitations of polar orbiting satellites have been recognized in measuring both algal bloom dynamics as well as turbidity fluctuations. Monitoring algal blooms, which are rapid rises in algal content, requires fine spatial (25 meters Lekki et al., 2019 or below Kutser, 2004) and temporal resolution (1 hr (Choi et al., 2014) data, as well as near real time data processing [Sawtell et al. 2019]. The fine spatial resolution is extremely important as the chlorophyll concentration can vary by as much as two orders of magnitude in just a few tens of meters Kutser, 2004. Even 30 meter data can be too coarse for sampling potentially harmful algae slicks, and can result in extreme underestimation of the chlorophyll content due to the spatial distribution of the bloom Kutser, 2004. In fact, researchers are calling for sub-meter resolution remote sensing data to study the impact of the fractal structure of the slicks on remote sensing products at coarser spatial resolutions [International Ocean-Colour Coordinating Group 2017]. Another example task that is limited by the current satellite-based ocean color sensor's spatio-temporal resolution combinations is the assessment of the spatial variability of turbidity in coastal regions on sub-meter scales [Luis et al., 2019]. A few specific examples where sub-meter data could be useful is in studying the impact of dredging Ehmann et al. 2019, river outflow IGOS, 2006, and glacial meltwaters Wójcik et al., 2019 on local turbidity. In summary, algae and turbidity are two optical constituents that require measurement on sub-meter scales to assess their regional impact.

\subsubsection{Spatio-temporal limitations of Satellite Deployment Approaches}

The orbital mechanics of polar orbiting satellites are the main factor limiting their spatial and temporal resolution combinations. Polar orbiting satellites follow Kepler's third law of planetary motion, which when combined with Newton's law of gravity follow:

$$
T^{2}=\frac{4 \cdot \pi^{2} \cdot r^{3}}{G \cdot M}
$$

where $\mathrm{T}$ is the orbital period (1.5 hours), $\mathrm{G}$ is the gravitational constant $\left(6.7 \cdot 10^{-11} \mathrm{~m}^{3}\right.$. $\left.\mathrm{kg}^{-1} \cdot \mathrm{s}^{-2}\right), \mathrm{M}$ is the mass of the earth $\left(6 \cdot 10^{24} \mathrm{~kg}\right)$, and $\mathrm{r}$ is the radius of the orbit $\left(6.7 \cdot 10^{6} \mathrm{~m}\right)$ 


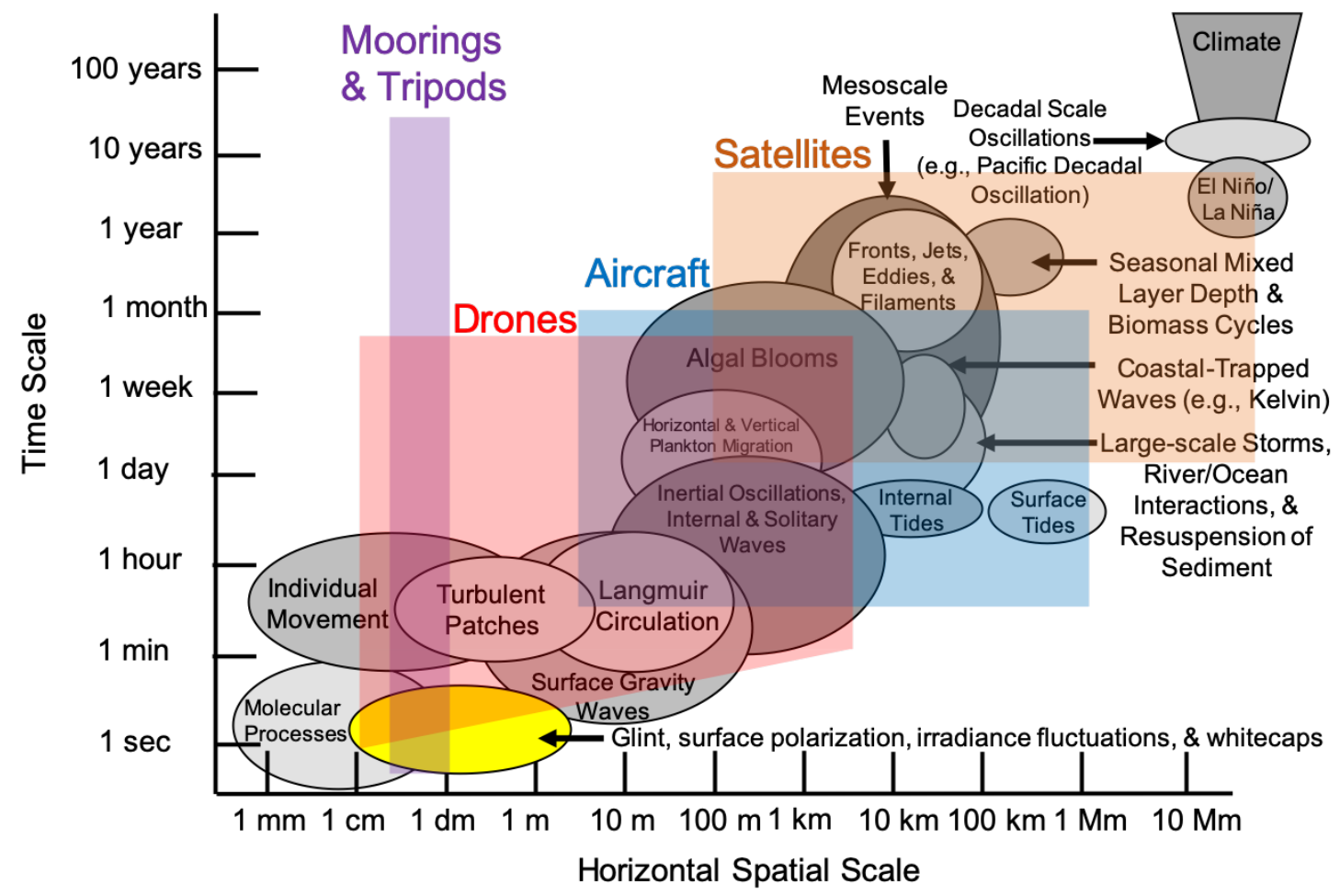

Figure 1-2: Physical and biological processes within the global oceans occur over a wide range of spatio-temporal resolution combinations. Satellites are able to collect global datasets over decadal timespans, but only at coarse resolutions. Aircraft are able to sense at finer spatial and temporal resolutions, but at the cost of limited temporal and spatial coverage. Drones can sense at sub-meter spatial scales, but sub-meter fluctuations in the surface characteristics (yellow circle) mar the radiometric data at these scales. This figure was adapted from IOCCG Report No. 12 International Ocean-Colour Coordinating Group, 2012b which was itself adapted from Dickey et al. [Dickey et al., 2006].

[Mobley et al., 2016a]. The orbital period combined with the swath width of the optical sensor limits the time required to resample the same region, resulting in the 2 day typical repeat cycle for polar orbiting satellites. The spatial resolution is limited by the amount of spatial smear induced by satellite velocity $(v)$ during exposure, as set by the orbital period:

$$
v=\frac{2 \cdot \pi \cdot r}{T}
$$

The typical satellite velocity is roughly $8 \cdot 10^{3} \mathrm{~m} \cdot \mathrm{s}^{-1}$. With this given velocity, the exposure time would be limited to roughly $10^{-4}$ seconds to achieve meter scale resolutions. This short of an exposure time is not sufficient to achieve the high SNR at the fine spectral resolution required for ocean color remote sensing. While this low exposure time is sufficient for panchromatic imagers imaging terrestrial scenes, it is not sufficient for multispectral or 
hyperspectral ocean color imagers which split the light into many more channels and require high SNRs. For the more typical km scale resolutions, the exposure time is limited to $10^{-1}$ seconds Mobley et al. 2016al. In summary, the orbital mechanics set the velocity, which limits the repeat cycle and spatial resolution (through the exposure time) of satellite ocean color remote sensors.

Changing the satellite remote sensing orbital characteristics and optics can overcome these limitations to some degree. Higher temporal coverage, at 1 hour sampling intervals of a single region, can be achieved by deploying satellites in geosynchronous orbits that match the rotation period of the earth. Geosynchronous orbits therefore sacrifice global coverage for temporal resolution. In one specific example, the increased temporal resolution provided by GOCI (Geostationary Ocean Color Imager, launched in 2010) enabled the study of a harmful algal bloom at hourly intervals, which revealed the relationship between water temperature and bloom formation in this region Choi et al., 2014]. If the spatial resolution is limited not by the exposure time, but by the number of pixels sampling the swath width, then either the number of pixels must be increased, or the swath width decreased, to increase the spatial resolution. Since increasing the number of pixels would decrease the measured signal per pixel (and therefore SNR), this solution is typically avoided for ocean color sensors. Instead the swath can be decreased, such as on Hyspiri which has a swath width of $150 \mathrm{~km}$ and achieves 60 meter resolution, but this limits the repeat cycle by about a factor of 10 (to 20 days) as more orbits must be completed to achieve global coverage [Jet Propulsion Laboratory, 2018]. Overall, although the optics and orbits of satellite sensors can be altered, the orbital mechanics still limit the achievable spatio-temporal combinations for sensing dark ocean color waters.

\subsubsection{Overview of Non-Satellite Platforms for Ocean Color Measurement}

Manned aircraft enable assessment of ocean properties at finer spatio-temporal resolution combinations, as they do not suffer from orbital mechanic limitations, but there are several practical and technical challenges associated with their use. Unlike satellites, aircraft can vary their speed, altitude, be easily reconfigured with different optical payloads, and can alter their flight paths. Aircraft can therefore achieve the appropriately high spatial (5-10 $\mathrm{m})$, temporal (selectable), and spectral $(5 \mathrm{~nm})$ resolution combinations by varying these operational parameters. Unfortunately, manned aircraft have a few significant disadvan- 
tages. Practically, manned aircraft have low availability Orcutt, 2012, require a dedicated pilot, and are quite costly for the researcher, at $\$ 350$ per square mile Chipman et al., 2009]. Technically, aircraft must correct for variable amounts of path radiance depending upon the sensors slant range (which is a function of altitude) and low frequency shifts in the reflected sky-light Kim et al. 2013. Due to their limited availability and significant technical challenges, ocean color sensors deployed on aircraft are therefore typically used for high impact applications such as testing satellite sensors at altitude and over specific regions, targeted studies of more complex regions in the ocean, and validating satellite products at finer spatial and temporal scales. While aircraft are often used for 2-10 meter resolution surveys of regions Green et al., 2001a; Fichot et al., 2016, Moses et al., 2012b; Hunter et al., 2010; Olmanson et al. 2013, they are rarely used at sub-meter spatial scales (though they have been used to achieve $20 \mathrm{~cm}$ resolution in at-least one case (Kim et al., 2013]) .

Low altitude platforms, such as towers, aerostats (blimps), and drones offer alternative low-altitude solutions to achieve sub-meter resolutions. Tower platforms, aerostats, and drones each fill a unique role in fine-scale remote sensing applications. Sensors deployed on tower platforms can collect long timeseries of a specific regions ocean color signal, as they can have power and data lines connected from shore. The timeseries, which would include data from a wide variety of oceanographic conditions, would be quite useful for validating instrumentation O'Shea et al. 2020, Gilerson et al., 2018. Additionally, instruments on tower platforms can be deployed in harsher conditions, such as during high wind events, than those deployed on aerial vehicles. Aerostats also provide a stable platform to collect fine-scale timeseries data of a particular region, but they can be deployed in regions without the infrastructure to support a tower. Aerostats could be particularly useful for measuring river outflow in Arctic regions, the impact of glacier melt waters on local primary production, and the effect of tidal cycles in remote coastal regions. Unlike towers and aerostats, drones can quickly respond to events and cover significant areas at meter scales Klemas, 2015. Although drones, can vary widely in size and operational ability (Table 1.4), the focus of the following sections is on Micro and Miniature drones. Micro and Miniature drones have the payload capacity to fly spectral cameras, the flight duration to cover several kilomters, and are light enough to be deployed by a single researcher. Drones are the most versatile low altitude platform due to their mobility, but their mobility is limiting and comes with additional complications. The additional complications include power and weight limitations 
of the electronic sensors and spatial resolution limitations due to spatial smearing of the measured water-leaving radiance signal. Drones are able to map and explore larger areas than the static low-altitude platforms, which is particularly useful for applications such as mapping harmful algal blooms along coastal shellfish beds and sediment plumes in estuaries after extreme weather events. Overall, towers, aerostats, and drones can each fill a specific niche for low-altitude sub-meter remote sensing, but drones are the most versatile and practically limiting of the low-altitude platforms.

The satellite ocean color remote sensing paradigm relies on many platform and task specific assumptions, which may not hold when remote sensing on meter or sub-meter resolutions. The deployment platform changes the remote sensing capabilities (e.g., spatial resolution), the weight and power limits of the camera (which are linked to the camera's sensitivity), the scientific resources that can be allocated to calibrating and testing the spectral cameras for ocean color remote sensing, and the pertinent environmental noise sources. While the broad effects of sensing on finer spatial scales than satellite sensors were covered previously (Table 1.1), a more detailed description of the changes required for the drone and aircraft remote sensing paradigms are outlined in the following sections (Tables 1.5, 1.6, 1.7. and 1.8). The focus of sub-meter remote sensing is from a drone based perspective, as operationally the drone is the most versatile low-altitude platform and practically it is the most limiting.

\begin{tabular}{|ccccccc|}
\hline $\begin{array}{c}\text { Drone } \\
\text { Class }\end{array}$ & Example & $\begin{array}{c}\text { Weight } \\
(\mathrm{kg})\end{array}$ & $\begin{array}{c}\text { Payload } \\
(\mathrm{kg})\end{array}$ & $\begin{array}{c}\text { Altitude } \\
(\mathrm{m})\end{array}$ & $\begin{array}{c}\text { Airspeed } \\
(\mathrm{m} / \mathrm{s})\end{array}$ & $\begin{array}{c}\text { Endurance } \\
(\mathrm{hr})\end{array}$ \\
\hline Nano & $\begin{array}{c}\text { Parrot } \\
\text { Rolling Spider }\end{array}$ & $0-1$ & 0.1 & 10 & 10 & 0.2 \\
Micro & DJI Phantom & $1-10$ & $<5$ & 400 & 50 & 0.5 \\
Miniature & $\begin{array}{c}\text { Boeing Scan } \\
\text { Eagle }\end{array}$ & $10-25$ & $<10$ & 1,000 & 125 & 4 \\
Medium & $\begin{array}{c}\text { Drone America } \\
\text { Phoebus }\end{array}$ & $25-600$ & $>10$ & $<5,500$ & $\sim 500$ & 12 \\
Large & Global Hawk & $>600$ & $>50$ & $>5,500$ & $\sim 500$ & 96 \\
\hline
\end{tabular}

Table 1.4: The available drones can be placed into classes based upon their operational capabilities, following a similar classification system as the U.S. Army U.S. Army UAS Center of Excellence, 2010]. This dissertation focuses on the Micro and Miniature classes of drones as they have sufficient payload for ocean color sensors and sufficient endurance for $\mathrm{km}$ scale ocean color missions, but can still be launched by a single researcher. 


\subsubsection{Observational Considerations}

Lower altitudes and velocities enable measurement on finer spatial and spectral resolution combinations for a given optical instrument, at the expense of area covered. Since the spatial resolution is often limited by spatial blurring from platform movement during exposure, slower velocities can enable longer integration times that achieve the same spatial resolution. While drones can only cover smaller regions $\left(1-100 \mathrm{~km}^{2}\right)$ due to their typically lower velocities and altitudes, the lower velocity and altitude enable them to achieve finer spatial resolutions $(<1 \mathrm{~m})$ at higher spectral resolutions $(2 \mathrm{~nm})$, as they can increase the exposure times to make up for the limited spectral bandwidth captured by the optical sensors (Table 1.5). Typical aircraft sensors must maintain a minimum velocity to stay airborne $\left(100-200 \mathrm{~km} \cdot \mathrm{h}^{-1}\right.$ at $0.6-5 \mathrm{~km}$ altitude), and satellite velocities are fixed based on their orbit $\left(27,000 \mathrm{~km} \cdot \mathrm{h}^{-1}\right.$ at $700 \mathrm{~km}$ ), clearly limiting their achievable spatial resolutions.

Unfortunately, the smaller drone platforms suffer from size, weight, and stability limitations. The camera systems available for drone based sensing must be less than $5 \mathrm{~kg}$ and must fit within the drone's small payload. The size and weight constraints limit the size of the optics and electronics that can be relative to those used on satellite sensors, therefore limiting the sensitivity of drone-borne ocean color imagers. The weight constraint also limits the amount of power that is available for the camera system (100 watts), while aircraft and satellite power availability no longer significantly limits the camera system. Finally, the smaller size platform also makes the system more susceptible to pitch and roll effects during flight than an aircraft, which will affect the geometry of the ocean color measurements and inhibit accurate georectification.

The reduction in the altitude, spatial resolution, area covered, and available power and weight will effect the sensitivity of the camera system that can be deployed (Table 1.6), the resources that can be dedicated to calibration (Table 1.7), the pertinent environmental factors (Table 1.8), and the assumptions that can be made when correcting for the environmental factors (Table 1.8 ).

\subsubsection{Camera and Imaging Considerations}

Unfortunately, the severe power and weight constraints on Micro and Miniature drones limit the sensitivity of the deployable cameras (Table 1.6). Commercially available drone-borne 


\begin{tabular}{|c|c|c|c|}
\hline Platform & \multirow{2}{*}{$\begin{array}{c}\text { Drone } \\
\text { (e.g., agriculture quadcopter) }\end{array}$} & \multirow{2}{*}{$\begin{array}{c}\text { Aircraft } \\
\text { (e.g., AVIRIS) }\end{array}$} & \multirow{2}{*}{$\begin{array}{c}\text { Satellite } \\
\text { (e.g., MODIS) }\end{array}$} \\
\hline Platform Limitations & & & \\
\hline Altitude & $0.1 \mathrm{~km}$ & $5 \mathrm{~km}$ & $700 \mathrm{~km}$ \\
\hline Velocity & $0-160 \mathrm{~km} \cdot \mathrm{h}^{-1}$ & $200-500 \mathrm{~km} \cdot \mathrm{h}^{-1}$ & $27,000 \mathrm{~km} \cdot \mathrm{h}^{-1}$ \\
\hline $\begin{array}{l}\text { Measurement } \\
\text { Schedule }\end{array}$ & $\begin{array}{l}\text { Selectable } \rightarrow \text { can respond to } \\
\text { events (weather dependent) }\end{array}$ & $\begin{array}{l}\text { Scheduling requirement } \rightarrow \\
\text { limited availability }\end{array}$ & $\begin{array}{l}\text { Orbitally fixed } \rightarrow \\
\text { aliasing effects }\end{array}$ \\
\hline Spatial coverage & $1-100 \mathrm{~km}^{2}$ & $100-10,000 \mathrm{~km}^{2}$ & Global \\
\hline Spatial resolution & Fine, 0.01-1 m, modular & Medium, 5-10's m, modular & Course, $10-1,000 \mathrm{~m}$, fixed \\
\hline Spectral Resolution & $2 \mathrm{~nm}$ (SNR permitting) & $5-10 \mathrm{~nm}$ & Course, $5-15 \mathrm{~nm}$ \\
\hline Temporal Resolution & Selectable! & Pilot limited & Orbitally set: $2-30$ days \\
\hline Cloud effects & $\begin{array}{l}\text { Fly under high altitude clouds, } \\
\text { fog blocks remote sensing }\end{array}$ & $\begin{array}{l}\text { Block remote sensing, } \\
\text { affect flight path }\end{array}$ & Block remote sensing \\
\hline Pitch/Roll effects & $\begin{array}{l}\text { Wind induced pitch and roll } \\
\text { smears ocean color, limits } \\
\text { georectification }\end{array}$ & Pitch and roll during turns & No atmospheric pitch/roll \\
\hline Camera weight limit? & $\begin{array}{c}\sim 5 \mathrm{~kg} \text { payload limit, } \uparrow \text { weight } \\
\downarrow \text { flight duration }\end{array}$ & $\begin{array}{c}\uparrow \text { Camera weight does not } \\
\downarrow \text { flight duration }\end{array}$ & $\begin{array}{c}\uparrow \text { Camera weight does not } \\
\downarrow \text { flight duration }\end{array}$ \\
\hline Camera power limit? & $\sim 100 \mathrm{~W}$ limit of batteries & Unlimited & Solar panel limited \\
\hline
\end{tabular}

Table 1.5: A comparison between the platform induced limitations of satellites, aircraft, and drones. Bold characteristics in the drone column may impact the validity of the satellite remote sensing paradigm on sub-meter estimates.

sensors are uncooled (due to weight and power constraints), have relatively low full-well capacities, and have slow optics. The combined effect of these camera characteristics is a limited dynamic range and signal-to-noise ratio, which disproportionately effects the lower intensity spectra of the ocean color signal. The increased noise in drone-borne camera systems can propagate to inaccuracy in fine-scale environmental noise correction and ocean color product estimation. Therefore, the available spectral camera systems must be assessed for retrieving ocean color products on sub-meter scales.

In contrast to drones, satellite and aircraft do not severely constrain the size and weight of their ocean color imagers, and are therefore able to achieve much more complex optical systems. These systems typically have fast and efficient optics as well as state-of-the art electronics with high full-well capacities, which set the dynamic range and shot-noise limited SNR. Specifically, the ocean color imagers on these platforms are able to use multiple stateof-the-art, large, heavy, and cooled camera systems, which are able to meet the high SNR requirements $(500-1,000)$ for wide dynamic range satellite ocean color remote sensing from high altitudes. 


\begin{tabular}{|c|c|c|c|}
\hline Platform & Drone & Aircraft & Satellite \\
\hline Camera Characteristics & (e.g., agricuiture quaccopter) & (e.g., AVIRIS) & (e.g., MODIS) \\
\hline $\begin{array}{l}\text { Can cameras be } \\
\text { cooled? }\end{array}$ & $\begin{array}{c}\text { No, limited by power and } \\
\text { weight }\end{array}$ & $\begin{array}{c}\text { Yes, power available to cool } \\
\text { cameras }\end{array}$ & $\begin{array}{l}\text { Yes, must be cooled due to } \\
\text { incident solar irradiance }\end{array}$ \\
\hline How is data offloaded? & $\begin{array}{l}\text { Limited on-board storage, } \\
\text { hard disk transferred }\end{array}$ & $\begin{array}{l}\text { Unlimited onboard storage, } \\
\text { hard disk transferred }\end{array}$ & $\begin{array}{l}\text { Limited onboard storage, then } \\
\text { telemetered to ground }\end{array}$ \\
\hline Full-well Capacity & $35 k$ & $100 k-800 k$ & $100 k-800 k$ \\
\hline $\begin{array}{l}\text { Shot-noise limited per- } \\
\text { pixel SNR }\end{array}$ & 180 & $300-900$ & $300-900$ \\
\hline $\begin{array}{l}\text { Ocean color SNR } \\
\text { requirement }\end{array}$ & Task dependent, undefined & $\sim 500-1,000$ & $\sim 500-1,000$ \\
\hline $\begin{array}{l}\text { Does sensor meet SNR } \\
\text { requirements? }\end{array}$ & $\begin{array}{c}\text { Unknown, limited sensor } \\
\text { testing }\end{array}$ & $\begin{array}{l}\text { Yes, validated by a team of } \\
\text { researchers }\end{array}$ & $\begin{array}{l}\text { Yes, validated by multiple } \\
\text { teams of researchers }\end{array}$ \\
\hline F\# & 2.4 & 1.6 & 1.6 \\
\hline Bits & $10-16$ & $12-16$ & $12-16$ \\
\hline Efficiency & $\sim 40 \%$ & $90 \%$ & $90 \%$ \\
\hline
\end{tabular}

Table 1.6: A comparison between the typical camera characteristics available for each platform. Bold characteristics in the drone column may impact the validity of the satellite remote sensing paradigm on sub-meter estimates.

\subsubsection{Calibration and Testing Considerations}

A major limitation of drone based remote sensing is that the researcher must maintain and validate their own camera system for specific ocean color remote sensing tasks (Table 1.7). The wide range of available camera systems remain untested in most sensing conditions, with only a sparse representation being used for regional low altitude ocean color applications, so researchers cannot rely on other published reports to determine if their specific system will work. The SNR requirements for specific tasks at these lower altitudes are poorly defined for low altitude measurements at sub-meter spatial scales. Instead of relying on previously published reports or the SNR of the camera to determine if it is useful for a task, the researcher must test the system during a field deployment to determine if the instrument and platform combination will be required to identify the accuracy limitations of the system. Overall, calibrating and testing each low-altitude camera system for a specific remote estimation task will introduce significant uncertainty into the results and put more of the burden on the ocean color scientists, slowing the adoption of this platform.

Satellite and aircraft based ocean color imager are calibrated and validated by independent research teams, separating this task from the ocean color scientist. The instrumentation can be calibrated and validated on a specific ocean color imager by one research team, and 
then a separate research team can use those calibration coefficients algorithms for future studies. This setup reduces the burden that falls upon the ocean color scientist, and reduces the uncertainty in the ocean color products. Unfortunately, since the areas that each sensor cover are much smaller for drones, the calibration and validation must be unique to each imager. Therefore, drone based imagers cannot utilize a similar separation of tasks during the calibration and testing phase.

\begin{tabular}{|c|c|c|c|}
\hline Platform & \multirow{2}{*}{$\begin{array}{c}\text { Drone } \\
\text { (e.g., agriculture quadcopter) }\end{array}$} & \multirow{2}{*}{$\begin{array}{c}\text { Aircraft } \\
\text { (e.g., AVIRIS) }\end{array}$} & \multirow{2}{*}{$\begin{array}{c}\text { Satellite } \\
\text { (e.g., MODIS) }\end{array}$} \\
\hline Calibration and Testing & & & \\
\hline Calibrating agency & Researcher (or manufacturer) & Research team & Space agency (e.g., NASA) \\
\hline $\begin{array}{l}\text { Cameras designed for } \\
\text { ocean color SNR req.? }\end{array}$ & $\begin{array}{c}\text { No, designed for terrestrial } \\
\text { targets }\end{array}$ & Yes, meets requirements & Yes, meets requirements \\
\hline $\begin{array}{l}\text { Sensors tested for } \\
\text { deriving ocean color } \\
\text { products? }\end{array}$ & No, limited testing & $\begin{array}{l}\text { Yes, researchers test for } \\
\text { sensing in specific regions }\end{array}$ & $\begin{array}{l}\text { Yes, researchers perform } \\
\text { validation on global scales }\end{array}$ \\
\hline $\begin{array}{l}\text { Req. stable radiometric } \\
\text { calibration? }\end{array}$ & No, can be recalibrated in lab & Yes, recalibrated seasonally & Yes, deployed for decades \\
\hline $\begin{array}{l}\text { New elements req. } \\
\text { additional calibration? }\end{array}$ & Yes, recalibration for polarizer & Yes, recalibration for polarizer & No, new elements not addec \\
\hline
\end{tabular}

Table 1.7: The burden of calibration and validation of drone-borne ocean color imagers currently falls upon the ocean color scientist. Requiring the ocean color scientist to calibrate and validate the products will introduce additional uncertainty and reduce productivity relative to the aircraft or satellite calibration model. Bold characteristics in the drone column may impact the validity of the satellite remote sensing paradigm on sub-meter estimates.

\subsubsection{Environmental Considerations}

The lower altitude and higher spatial resolution achieved by drone-borne ocean color imagers changes the relevant environmental noise sources and their characteristics, relative to the satellite ocean color remote sensing paradigm. Drone-based ocean color imagers are most effected by fluctuations due to glint on sub-meter spatial scales. Capillary waves and heterogeneous sky conditions limit the validity of the typical assumptions used during glint correction for the sub-meter spatial resolutions achievable from drone platforms (Table 1.8). The capillary waves change the reflectance factor and polarization state on sub-meter scales, potentially inducing changes in ocean color products if not algorithmically corrected. The typical sky glint correction approaches leverage either the reflectance factor to subtract the downwelling sky radiance Mueller and Austin, 1995 or a polarizer to remove the vertically polarized reflected skylight Fougnie et al., 1999]. The heterogeneous sky will reflect unique 
contributions of downwelling skylight into the sensor, which cannot be collected by a single upward facing radiometer. The differences in the environmental factors and correction techniques require further introspection to be able to successfully leverage the traditional glint correction approaches for ocean color product retrieval.

Ocean color imagers on aircraft and satellite are mainly affected by path radiance instead of glint. At 0.01-1 kilometer scale spatial resolutions, the surface reflected light can be approximated as coming from a specific portion of the sky and being vertically polarized at specific view angles. While the surface reflected light is relatively straightforward to correct for, and can be done using look up tables given the local windspeed, the path radiance can comprise nearly $85 \%$ of the environmental noise on satellite and aircraft based sensors Orcutt, 2012, due to their high altitudes. Since a significant fraction of the signal on these sensors is due to path radiance, the ocean color sensors must maintain high SNR values to accurately estimate the ocean color signal.

\subsubsection{State-of-the-Art for Sub-Orbital Optical Constituent Mapping}

The above material covered the changes required for collecting meter and sub-meter data using the aircraft and drone ocean color remote sensing paradigms, and the following material will introduce the applications of these platforms at these scales. Aircraft were the preliminary test platform to determine if remotely measured changes in the ocean's color could be related to changes in the optical constituents. In 1970, a custom off-plane Ebert point spectrometer, with 5-7.5 $\mathrm{nm}$ bandwidth and 400-700 $\mathrm{nm}$ spectral range, was flown on an aircraft at an altitude of $305 \mathrm{~m}$ over ocean waters with a wide range of chlorophyll concentrations Clarke et al. 1970. The variations in the ocean color signal in the blue, green, and red measured from an aircraft were successfully correlated with changes in the chlorophyll concentration taken aboard a ship following the same track. Also, path radiance was noticed as a significant environmental source of noise, with the path radiance increasing the total measured signal by a factor of 5 when the altitude was varied from 200 to 3,000 meters. A spectrometric removal procedure was suggested, though not demonstrated, to be able to remove the path radiance from high altitude measurements made from a satellite. The research also identified surface reflected light (glint) and other optical constituents as potential sources of error when estimating chlorophyll. The initial assessment of remote estimation of chlorophyll informed the myriad of areas that required further advancement 


\begin{tabular}{|c|c|c|c|}
\hline Platform & \multirow{2}{*}{$\begin{array}{c}\text { Drone } \\
\text { (e.g., agriculture quadcopter) }\end{array}$} & \multirow{2}{*}{$\begin{array}{c}\text { Aircraft } \\
\text { (e.g., AVIRIS) }\end{array}$} & \multirow{2}{*}{$\begin{array}{c}\text { Satellite } \\
\text { (e.g., MODIS) }\end{array}$} \\
\hline Environmental Factors & & & \\
\hline Spatial resolution & $\begin{array}{c}\text { Fine resolution, } 0.1-1 \mathrm{~m} \text {, } \\
\text { low altitude }\end{array}$ & $\begin{array}{l}\text { Medium resolution, } 5-10 \text { 's m, } \\
\text { medium altitude }\end{array}$ & $\begin{array}{c}\text { Course resolution, } 10-1000 \mathrm{~m} \text {, } \\
\text { high altitude }\end{array}$ \\
\hline $\begin{array}{l}\text { Path radiance } \% \text { of } \\
\text { total radiance }\end{array}$ & $<5 \%$ & $\begin{array}{l}5-85 \% \text {, dominates at high } \\
\text { altitudes }\end{array}$ & $85 \%$ (including glint) \\
\hline $\begin{array}{l}\text { Glint contribution to } \\
\text { total radiance }\end{array}$ & $\mathbf{5 0} \%$, varies spectrally & $\begin{array}{c}\text { Significant at } \downarrow \text { altitudes, } \\
\text { varies spectrally }\end{array}$ & $\ll$ path radiance \\
\hline $\begin{array}{l}\text { Environmental noise } \\
\text { correction assumptions }\end{array}$ & $\begin{array}{l}\text { Vertically polarized reflected } \\
\text { light, constant reflectance } \\
\text { value, origin of reflected light }\end{array}$ & $\begin{array}{l}\text { Dark Pixel, constant } \\
\text { reflectance value }\end{array}$ & $\begin{array}{l}\text { Dark Pixel, constant } \\
\text { reflectance value }\end{array}$ \\
\hline $\begin{array}{l}\text { Is reflectance value } \\
\text { constant? }\end{array}$ & $\begin{array}{l}\text { No, capillary waves alter } \\
\text { reflectance at sub-meter } \\
\text { scales }\end{array}$ & $\begin{array}{l}\text { Mostly, capillary waves } \\
\text { averaged by meter scale }\end{array}$ & $\begin{array}{l}\text { Yes, kilometer scale averages } \\
\text { waves, wind dependent } \\
\text { reflectance }\end{array}$ \\
\hline $\begin{array}{l}\text { Does reflected skylight } \\
\text { come from a single } \\
\text { region of the sky? }\end{array}$ & Not if sky is partially cloudy & $\begin{array}{l}\text { Maybe, surface can mostly be } \\
\text { assumed planar }\end{array}$ & $\begin{array}{l}\text { Yes, surface can be assumed } \\
\text { planar at km scale }\end{array}$ \\
\hline $\begin{array}{l}\text { Can the glint's } \\
\text { polarization state be } \\
\text { approximated by } \\
\text { Fresnel reflectance? }\end{array}$ & $\begin{array}{l}\text { No, cannot assume a planar } \\
\text { surface (capillary waves) }\end{array}$ & $\begin{array}{c}\text { Probably, meters scale } \\
\text { averages wave effects, but } \\
\text { atmosphere polarizes light }\end{array}$ & $\begin{array}{l}\text { Yes, km scale averages waves, } \\
\text { but atmosphere polarizes }\end{array}$ \\
\hline $\begin{array}{l}\text { How do cloud shadows } \\
\text { limit measurement? }\end{array}$ & $\begin{array}{l}\text { Can fly irradiometer beneath } \\
\text { clouds to estimate remote } \\
\text { sensing reflectance }\end{array}$ & $\begin{array}{l}\text { Can fly irradiometer beneath } \\
\text { clouds to estimate remote } \\
\text { sensing reflectance }\end{array}$ & $\begin{array}{c}\text { Significantly alter products if } \\
\text { uncorrected }\end{array}$ \\
\hline $\begin{array}{l}\text { How do white caps } \\
\text { effect measurement? }\end{array}$ & $\begin{array}{c}\text { Block remote sensing } \\
\text { estimates in that fine spatial } \\
\text { region }\end{array}$ & $\begin{array}{l}\text { Averaged into measurement, } \\
\text { algorithmically removed }\end{array}$ & $\begin{array}{l}\text { Averaged into measurement, } \\
\text { algorithmically removed }\end{array}$ \\
\hline
\end{tabular}

Table 1.8: A comparison between the environmental factors effecting ocean color measurements from satellites, aircraft, and drones. Bold characteristics in the drone column may impact the validity of the satellite remote sensing paradigm on fine-scale estimates.

for the accurate remote estimation of ocean color products.

The next major advance in low-altitude applications in ocean color remote sensing occurred in 1988, with NASA's Airborne Visible-Infrared Imaging Spectrometer (AVIRIS) (Table 1.9, 2nd column). AVIRIS was used to truth future satellites, with a focus on terrestrial applications. AVIRIS's initial SNR characteristics were too low for sensitive ocean color measurement, as the instrument was designed for terrestrial sensing, however significant spatial binning was sufficient to increase the SNR to usable levels for ocean color remote sensing [Carder et al., 1993]. The instrument's spatial resolution was reduced due to binning from $20 \mathrm{~m}$ to $280 \mathrm{~m}$ at red wavelengths, and from 20 meters to $1,000 \mathrm{~m}$ at blue wavelengths. With the increased SNR, ocean color scientists were able to map CDOM and SPM, by using absorption at $415 \mathrm{~nm}$ and backscattering at $671 \mathrm{~nm}$ as indicators, in a unusual nearshore plume in Tampa Bay. After initial testing, AVIRIS underwent many electronic 


\begin{tabular}{|c|ccc|}
\hline \multicolumn{1}{|c|}{ imager } & AVIRIS & PHILLS & PRISM \\
\cline { 1 - 3 } $\begin{array}{c}\text { Characteristics } \\
\text { Weight }(\mathrm{kg})\end{array}$ & $340 \mathrm{~kg}$ & $\sim 5 \mathrm{~kg}$ & $40 \mathrm{~kg}$ \\
Size $(\mathrm{m})$ & $2.0 \times 1.0 \times 1.0$ & $0.4 \times 0.15 \times 0.2$ & $0.6 \times 0.6 \times 0.6$ \\
Aperture & $10 \mathrm{~cm} \times 20 \mathrm{~cm}$ mirror & $\mathrm{f} / 2$ & $\mathrm{~F} / 1.8$ \\
$\begin{array}{c}\text { Radiometric } \\
\text { Resolution (bits) } \\
\begin{array}{c}\text { Spectral } \\
\text { Resolution } \\
\text { SNR }\end{array}\end{array}$ & $10 \rightarrow 12 \rightarrow 16$ & 14 & 14 \\
\hline
\end{tabular}

Table 1.9: Airborne ocean color imagers advanced significantly over the past few decades. The size and weight of the airborne imagers has decreased, while their optical efficiency and spectral resolution has dramatically increased. Unfortunately, the airborne ocean color imagers are too large for deployment on the available drone platforms. Mouroulis et al., 2014: Kramer, Green et al., 2001b a; Dickey et al., 2006; Davis; Davis et al., 2002]

and optical retrofits that increased the SNR to the point that it could be used for chlorophyll estimation in dark Case I waters Hamilton et al., 1991. Additionally, the spectrometric atmospheric removal algorithm (ATREM) was developed to enable accurate estimation and removal of the significant path radiance within the signal Gao et al. 2000. In addition to basic mapping tasks, AVIRIS has also been deployed to calibrate ocean color satellites, such as the Ocean Color and Temperature Scanner (OCTS), for changes that occurred due to the launching of the satellite to orbit and due to the differences in the radiation and temperature while in orbit [Green et al., 2001b]. In addition to calibrating ocean color satellites, AVIRIS has also been used to determine the ideal bands for the remote estimation of chlorophyll, CDOM, and SPM products from MODIS and MERIS in an estuary Lunetta et al. 2009. The evolution of the sensor sensitivity, atmospheric removal algorithms, and ocean color algorithms by different teams has allowed AVIRIS to become a versatile airborne sensor for ocean color remote sensing applications.

A major problem with AVIRIS is that it was not originally designed nor intended for high sensitivity ocean color research. Ocean color scientists developed their own sensor, Ocean Portable Hyperspectral Imager for Low-Light Spectroscopy (PHILLS) (Table 1.9. 3rd column), specifically for ocean color remote sensing. The commercial availability of the components allowed multiple sensors to be constructed for oceanographic applications Davis et al., 2002. Ocean PHILLS has been used in optically complex regions for bathymetry 
Louchard et al., 2003, bottom type estimation LLouchard et al., 2003, and leaf area index of benthic seagrass Dierssen et al., 2003. Ocean PHILLS eventually evolved into the Spectroscopic Aerial Mapping System with On-board Navigation (SAMSON) sensor. SAMSON informed users that the spatial, spectral, and temporal resolution needed to be increased for coastal regions [Davis et al., 2007]. In addition to providing a commercially assemble-able system to meet the needs of ocean color scientists, a higher sensitivity airborne hyperspectral imager was also developed and maintained by NASA specifically for coastal ocean color remote sensing applications. PRISM (Portable Remote Imaging Spectrometer Mouroulis et al., 2014, Table 1.9, fourth column), the latest ocean color hyperspectral imager from NASA, has been used for targeted assessment of floating seagrass as well as other ocean color monitoring programs Dierssen et al., 2015. Clearly, aircraft based imagers fill a sampling niche for high spatial, spectral, and temporal resolution data at specific time intervals for large-scale oceanographic projects.

While the history and expertise of using advanced ocean color imagers on-board aircraft for 10 meter scale ocean color remote sensing and satellite validation stretches back 4 decades, the use of lightweight multispectral cameras for sub-meter ocean color product retrieval from drones only stretches back a few years. One of the the seminal assessments of a droneborne multispectral camera for simultaneously estimating multiple water quality parameters occurred in 2015. In this study, Secchi disk depth, total phosphorus, and chlorophyll a were all estimated in a bright reservoir in Kinmen, Taiwan. The researchers created an environmental noise (e.g., surface reflected light) removal algorithm which they applied during the regression training process to achieve the highest accuracy estimates. The finescale fluctuation in the data were present in other data sets, which resulted in the creation of spatial filtering algorithms to eliminate the sub-meter fluctuations in the the field estimated SPM, chlorophyll, and turbidity [Totsuka et al., 2019]. In summary, the lightweight cameras that can be flown on drones are able to estimate specific optical constituents in near ideal conditions, despite the sub-meter environmental noise sources and low SNRs by leveraging spatial filtering.

The drone ocean color remote sensing paradigm has advanced beyond retrieving estimates of surface optical constituent concentrations from ocean color algorithms. The standard band ratio based ocean color algorithms were unable to achieve sufficient accuracy for SPM estimation from low-altitude multispectral cameras, so instead artificial neural net- 
works (ANNs) were implemented to increase the SPM estimation accuracy for a specific region Guimarães et al., 2019. In addition to being able to retrieve ocean color products on the surface, spectral algorithms were also proven to retrieve SPM products at multiple depths from drone-borne multispectral cameras Larson et al., 2018. Finally, lightweight multispectral cameras were used to estimate both optically active and non-optical constituents in the water column, such as nitrogen and phosphorus, at fine scales Arango and Nairn, 2019]. The range of ocean color remote sensing applications has moved beyond using ocean color algorithms for estimation of optical constituents in surface waters.

A limited number of studies also used lightweight hyperspectral cameras to examine the ocean's color from low-altitude platforms. A snapshot hyperspectral imager was deployed from tower platforms in multiple locations to study glint variations on fine spatial scales Gilerson et al., 2018; Carrizo et al., 2019. This imager quantified the variations in the reflection coefficient on sub-meter scales at a wide range of view angles and solar positions, due to wind speed. The standard deviaition of the reflection coefficient is as high as $35 \%$ for windspeeds under $5 \mathrm{~m} \cdot \mathrm{s}^{-1}$, and more than $100 \%$ at higher windspeeds and view angles Carrizo et al., 2019. In addition to using hyperspectral cameras to study variations in the ocean color signal, a pushbroom hyperspectral imaging spectrometer was also deployed from a drone to map cyanobacteria within the Daechung reservoir in South Korea Kwon et al. 2020. A spatial filtering method removed environmental noise fluctuations in the $20 \mathrm{~cm}$ resolution ocean color data. The hyperspectral camera system successfully mapped depth integrated phycocyanin. Unfortunately, the same algorithm struggled to recover phycocyanin at specific depths using the same band ratio. Overall, there are a limited number of studies testing lightweight hyperspectral imaging spectrometers, as the imagers are only now becoming widely available for ocean color remote sensing.

\subsubsection{Identified Limitations in Fine-scale Optical Constituent Estimation}

Factors that currently limit the use of lightweight spectral cameras on drones fall into two main categories: practical limitations due to the platform, and optical limitations due to changes in the environmental optics and sensor payload. The first platform limitation that comes to mind for small drones is wind induced pitch and roll variations. These changes in the pitch and roll can lead to measurement of the ocean color signal and incident irradiance at fluctuating view angles [Shang et al., 2017], which require correction. Additionally, the 
images taken under pitch and roll effects require image processing based georectification before they can be used to map the ocean color signal. However, unlike terrestrial scenes, the dynamics of the ocean's surface limit the accuracy of image processing based georectification algorithms on images lacking land or static ground control points $\mathrm{Su}, 2017$, Guimarães et al. 2019 Larson et al. 2018]. While the practical problems are important for all drone based mapping, their impact can be reduced through advancing the platform; either increasing the orientation measurement accuracy of the platform or increasing the platform stability. The second category of limitations, optical limitations, is specific to sensing the ocean color signal. A few region specific optical limitations include scattering by the seafloor Zeng et al. 2017], tree shadows reducing the incident irradiance [Guimarães et al., 2019], and urban objects blocking the ocean color signal [Larson et al., 2018]. However, the impact of these features is limited to small spatial regions of very nearshore applications. The more influential optical limitations for ocean color sensing, which this dissertation investigates further, include: sub-meter glint induced variations in the oceans color, the limited spectral resolution of the available imagers, and the impact of electronic noise sources on the ocean color products.

Surface reflected light (glint) is the major limiting environmental noise source in submeter applications. In the initial assessments of multispectral cameras from drones, wind driven capillary waves induced severe variations in the glint, which created fluctuations in the ocean color products on sub-meter scales that did not exist within the surface waters Zeng et al., 2017; $\mathrm{Su}, 2017$. A few glint removal algorithms were created to correct for this finescale glint issue in multispectral images collected from drones, but each relied upon spatial filtering. One algorithm was based upon median filtering, innately trading spatial resolution for ocean color product estimation accuracy [Totsuka et al. 2019]. Another algorithm, the matching pixel by pixel algorithm, removed glint fluctuations during empirical algorithm training by iterating through combinations of pixels in the training images to find the most accurate regression $\mathrm{Su}, 2017]$. A previously proven spatially filtering algorithm, SavitzkyGolay filtering, was also used to reduce the fluctuations induced by the surface reflected light Kwon et al., 2020]. Consequently, the traditional glint removal approaches typically used for ocean color measurement must be assessed on sub-meter scales, to achieve higher resolution estimates than the spatial filtering glint correction algorithms currently provide.

An additional limitation of the currently tested sensors is that complex ocean color 
product retrieval tasks require more spectral bands than are currently provided. In one study, a multispectral camera deployed on a drone was unable to achieve the same accuracy as a hyperspectral spectroradiometer for multi-depth SPM estimation [Larson et al., 2018]. While a hyperspectral camera was used for multi-depth estimation of a common pigment for harmful algae, phycocyanin, the three band algorithm used on the hyperspectral data poorly estimated the phycocyanin at multiple depths. Although lightweight hyperspectral cameras could provide the additional spectral bands required to increase the accuracy of multidepth estimation tasks, the finest spectral resolution of the commercially available lightweight hyperspectral cameras $(2 \mathrm{~nm})$ is not sufficient for all tasks. For example, the validation of the satellite-borne ocean color imager (OCI), which is part of the Plankton, Aerosol, Cloud, Ocean Ecosystem (PACE) mission, requires $1 \mathrm{~nm}$ spectral resolution ocean color measurements [Zibordi et al., 2017]. Accordingly, the spectral resolution of the currently available hyperspectral cameras must be increased through the use of custom cameras Sigernes et al. 2018 or novel data processing approaches (e.g., spectral super-resolution), before they can be used for satellite vicarious calibration from ships.

A final limitation of drone-borne sensors is that the optoelectronics and photonics noise sources will limit the radiometric accuracy of the hyperspectral imagers required to retrieve more complex ocean color products. The currently available hyperspectral cameras have significantly lower signal-to-noise ratios (SNR) and dynamic ranges than hyperspectral cameras typically used in aircraft or satellite based ocean color remote sensing O'Shea et al., 2020. International Ocean-Colour Coordinating Group, 2012a. The real-world SNR is even lower than the theoretical value on hyperspectral cameras because they simultaneously image the entire spectrum, so the lowest intensity spectra will have a lower SNR than the highest intensity pixel O'Shea et al., 2020, O'Shea and Laney, 2020]. The low SNR can significantly alter the accuracy of the computed ocean color products, either through the ocean color algorithms directly or through the environmental noise correction algorithms Levin et al., 2005, Levin and Levina, 2007; Moses et al., 2012a, 2015. Therefore, the impact of optoelectronic and photonic noise needs to be considered in the most sensitive ocean color applications before using lightweight hyperspectral cameras to map optical constituents. 


\subsection{Dissertation Overview}

Each of the chapters within this dissertation focuses on the optical considerations necessary to adapt a key step within the satellite ocean color remote sensing paradigm to accurately measure hyperspectral data at sub-meter spatial scales from low altitude platforms over water bodies. Specifically, each chapter focuses on one of the limitations in fine-scale optical constituent estimation previously discussed in section 1.3.8. The chapters are laid out as follows:

Chapter 2 Overview: Chapter 2 explores the limitations of three traditional glint correction approaches for use on fine spatial scales through a field deployment. At centimeter scales, capillary wave induced fluctuations in the ocean's surface may invalidate assumptions in the glint correction approaches, such as the polarization state at Brewster's angle and the value of the reflection coefficient. Of the three traditional algorithms tested during the field deployment, the Lee et al. [Lee et al., 2010] glint correction approach most accurately recovered centimeter scale optical constituent concentrations [O'Shea et al., 2020]. However, the polarization based approach matched the chlorophyll estimation accuracy of the Lee et al. correction, without the same technical requirements (e.g., additional spectral bands and radiometer).

Chapter 2 Contributions: The optical considerations discussed in Chapter 2 enable sensing despite fluctuations in the sky and solar glint due to fine-scale capillary waves (previously discussed in section 1.3.8. First, with the assessed viewing geometry and glint correction approaches, researchers can now collect accurate sub-meter data even when capillary waves are present (i.e., when there is wind) and the sun is near zenith. Second, the sub-meter scale glint correction process no longer needs to sacrifice spatial resolution to correct for glint, thereby increasing the area that can be covered while maintaining the desired spatial resolution.

Chapter 3 Overview: Chapter 3 demonstrates a simulation framework for assessing the photonic and optoelectronic induced limitations on the ocean color products estimated from lightweight imaging spectrometers in non-ideal sensing scenarios O'Shea and Laney, 2020]. In the first application, the simulation framework provides quantitative accuracy metrics to compare between specific camera systems for chlorophyll fluorescence line height estimation in a simulated coastal region. In the second application, the framework identifies 
dynamic range limitations with currently available hyperspectral imagers for simultaneously imaging biological productivity in Antarctic waters and sea ice. By identifying the dynamic range limitation, an engineering solution to that problem was hypothesized without having to deploy the system in that region.

Chapter 3 Contributions: To date, applications of fine-scale ocean color product retrieval has largely focused on ideal sensing scenarios using multispectral cameras (section 1.3.7), where optoelectronic and photonic noise sources were minor issues compared to glint correction. The simulation framework provides researchers with a quantitative means of selecting cameras, developing algorithms, and engineering solutions for accurate ocean color product retrieval in non-ideal scenarios, without the need for deployment in these regions.

Chapter 4 Overview: Chapter 4 develops a new machine learning based data processing approach to overcome hardware induced limitations on the spectral resolution of a grating-based imaging spectrometer. The spectral super-resolution approach uses the sub-pixel spectral shift in spatially oversampled images taken by a commercially available imaging spectrometer as the non-redundant information required to increase the spectral resolution beyond the pixel-limited value. We demonstrate that by leveraging the spectral shift, an imaging spectrometer can estimate frequencies beyond the Nyquist for data with similar statistics as the training dataset, despite environmental and optoelectronic noise sources characteristic of ocean color remote sensing. For testing datasets with substantially different statistics from the training set, such as laboratory generated ocean color datasets, frequencies up to and slightly past the Nyquist are better represented, at the cost of additional high frequency noise which reduces the overall accuracy of the prediction.

Chapter 4 Contributions: In the satellite ocean color remote sensing paradigm, oversampled homogeneous ocean color regions would have been binned to increase the signalto-noise ratio, but this chapter introduces an alternative approach to instead improve the spectral resolution by leveraging the spatial homogeneity. The spectral super-resolution technique weakly generalized to ocean color datasets, but further increasing the generalizability of the technique by training on higher spectral resolution ocean color datasets could provide the spectral resolution required for the vicarious calibration of next generation ocean color satellites Zibordi et al. 2017. Outside of the ocean optics domain, this technique could relax the spectral resolution requirement for many industrial and agricultural applications that spatially over-sample the target, such as conveyor belt based identification of materials. 
Chapter 5 Overview: Chapter 5 first summarizes the individual contributions of each chapter to advancing the sub-meter ocean color remote sensing paradigm and then demonstrates how each step in the paradigm benefits successive steps through an example sub-meter ocean color application. Additionally, Chapter 5 covers non-standard ocean color applications that benefit from this research, including applications that leverage aerostats and towers as platforms. Finally, this chapter discusses the future research required to advance the presented sub-meter ocean color remote sensing paradigm to fully leverage imaging spectrometers to map optical constituents from low altitude platforms in optically complex regions. 


\title{
Chapter 2
}

\section{Evaluation of glint correction approaches for fine-scale ocean color measurements by lightweight hyperspectral imaging spectrometers}

This chapter was published in the Optical Society of America's Journal of Applied Optics O'Shea et al. 2020 and a reformatted version is reproduced here.

\begin{abstract}
Low-power, lightweight, off-the-shelf imaging spectrometers, deployed on above-water fixed platforms or on low-altitude aerial drones, have significant potential for enabling fine-scale assessment of radiometrically-derived water quality properties (WQPs) in oceans, lakes, and reservoirs. In such applications it is essential that the measured water-leaving spectral radiances be corrected for surface-reflected light, i.e. glint. However, noise and spectral characteristics of these imagers, and environmental sources of fine-scale radiometric variability such as capillary waves, complicate the glint correction problem. Despite having a low signal-to-noise ratio, a representative lightweight imaging spectrometer provided accurate radiometric estimates of chlorophyll concentration - an informative WQP - from glint-corrected hyperspectral radiances in a fixed-platform application in a coastal ocean region. Optimal glint correction was provided by a spectral optimization algorithm, which outperformed both a hardware solution utilizing a polarizer and a subtractive algorithm incorporating the reflectance measured in the near-infrared. In the same coastal region this spectral optimization approach also provided the best glint correction for radiometric estimates of backscatter at $650 \mathrm{~nm}$, a WQP indicative of suspended particle load.
\end{abstract}




\subsection{Introduction}

\subsubsection{Motivation}

Commercially available imaging spectrometers are now low-power, lightweight, and inexpensive enough to find wider application in environmental monitoring. Such imagers have been used in terrestrial applications on small aerial drones to provide spectral reflectance imagery that remote sensing algorithms can in turn utilize to map plant biomass Honkavaara et al. 2013; Aasen et al., 2015; Zhu et al., 2018; Sankey et al., 2017] and distributions of terrestrial plant phenotypes Behmann et al. 2018 or tree species [Dalponte et al., 2012, all on fine spatial scales. Analogous environmental radiometry of aquatic systems (i.e., oceans, rivers, and reservoirs) using fixed-platform or low-altitude drones has to date instead relied primarily on multispectral cameras, not hyperspectral ones, to provide fine-scale spatial insight into distributions of water quality properties (WQPs) that can be derived radiometrically Choo et al. 2018; $\mathrm{Su}, 2017 \mid$. The multispectral approaches currently used in aquatic systems are roughly comparable to the state-of-the-art seen in satellite-based ocean color remote sensing of roughly a decade ago. In principle, newer imaging spectrometers can be used with recently developed hyperspectral algorithms to estimate a much broader range of WQPs from measured hyperspectral water-leaving radiance spectra [e.g., Defoin-Platel and Chami, 2007; Lubac et al., 2008, Aiken et al., 2014. These hyperspectral algorithms rely on accurate estimation of the water-leaving radiance and so require correction for surface-reflected light (i.e., glint) |Mobley, 1999; Lee et al., 2010|: a largely unavoidable component of upwelling radiance spectra whose effect must be minimized in order to maximize accuracy of WQPs estimated radiometrically from natural water bodies.

Aquatic applications of such lightweight, low-power imaging spectrometers are not directly analogous to terrestrial applications in several respects. First, aquatic water-leaving radiance reflectances are typically much lower than those of comparable terrestrial scenes and so the signal-to-noise (SNR) characteristics of the imager are of primary importance. Second, glint correction is a particular aquatic challenge which requires simultaneous measurement of additional radiances in the NIR, beyond those needed to compute a particular WQP of interest (Fig. 2-1). These measured NIR radiances may exhibit a lower SNR than the visible radiances, due to a combination of the spectral efficiency of the imager (i.e., the spectral efficiency of the optics and the quantum efficiency of the detector) and a lower ra- 


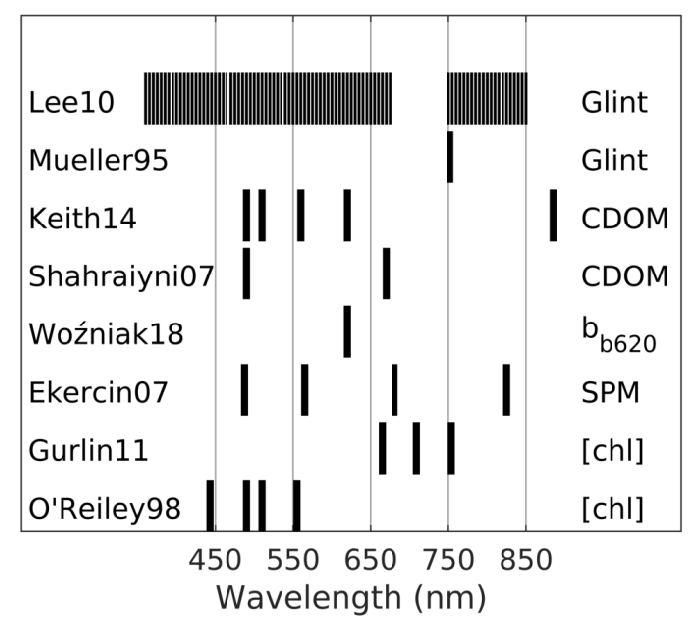

Figure 2-1: Center wavelengths used in common algorithms for various WQPs Lee et al., 2010; Mobley, 1999; Mueller and Austin, 1995; Shahraiyni et al., 2007; Keith et al., 2014; Ekercin, 2007; Woźniak et al., 2018; Gurlin et al., 2011; O'Reilly et al., 1998; Gholizadeh et al., 2016]. Highest accuracy algorithms often utilize a larger number of wavelengths. The gap in the waveband set for Lee10 (a glint correction algorithm) indicates wavelengths omitted surrounding the chlorophyll fluorescence emission. Abbreviations: CDOM - colored dissolved organic matter; $\mathrm{b}_{\mathrm{b} 620}$ - optical backscatter at $620 \mathrm{~nm}$; SPM - suspended particulate material; [chl] - chlorophyll concentration.

diance in the NIR relative to the visible emanating from aquatic bodies Moses et al., 2012a; $\mathrm{Su}$, 2017]. Since the shot-noise limited SNR is inversely proportional to the measured signal, only the radiance with the highest intensity measured signal (green) can reach the maximum possible SNR. Although in fine-scale terrestrial applications imagers with different exposures could simultaneously collect high SNR visible and NIR radiances, at sub-meter spatial scales natural water bodies can exhibit strong spatial and temporal variability due to glint, which necessitates the simultaneous collection of radiances at all pertinent wavelengths. Additionally, the fine-scale fluctuations in the reflectance may limit the effectiveness of software glint correction algorithms that assume a specific, fixed reflectance character. Lastly, although glint is strongly polarized at particular observation angles, simply affixing a polarizer to the imaging spectrometer will not be sufficient without understanding how any particular WQP estimate is jointly affected by the change in the spectral efficiency (and therefore spectral SNR) due to the polarizer and the capillary wave-induced fluctuations in the polarization of the surface reflected light. It is reasonable to expect that the combined effect of these various factors may lead to certain correction approaches outperforming others, which is an important factor to examine before applying terrestrial-oriented imaging approaches to 


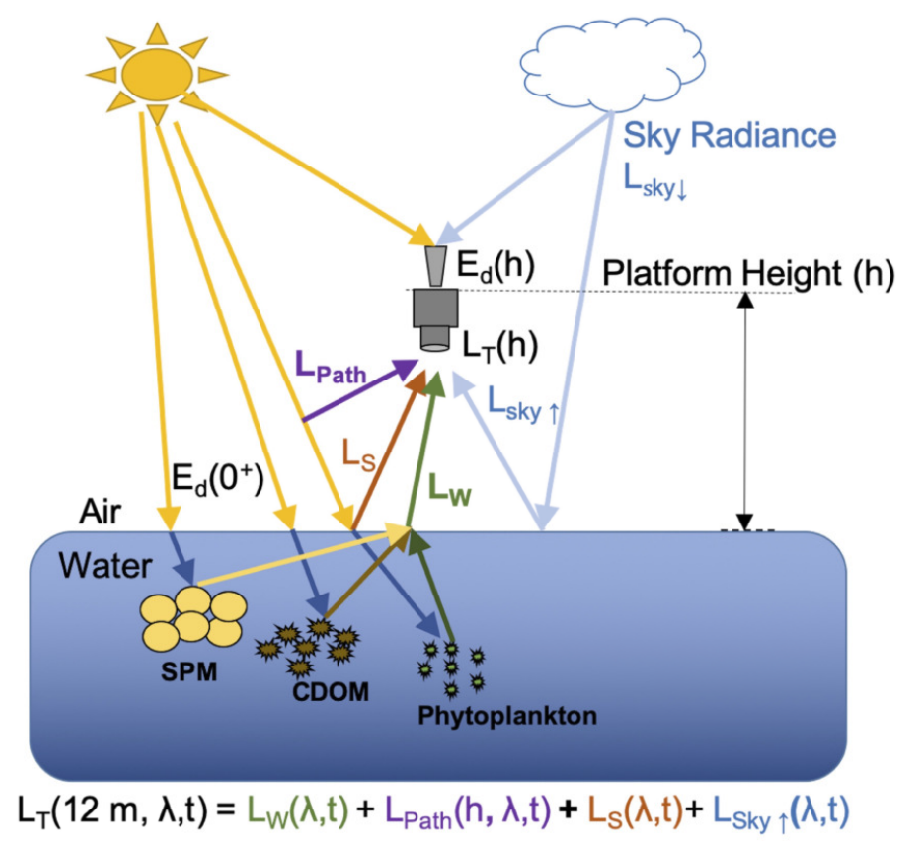

Figure 2-2: Suspended particulate matter (SPM), colored dissolved organic matter (CDOM), and phytoplankton are optically active WQPs that shape the water-leaving radiance $\left(\mathrm{L}_{\mathrm{W}}\right)$ in optically deep water bodies. Accurately estimating their concentrations from a ratio of the total at-sensor spectral radiance $\left[\mathrm{L}_{\mathrm{T}}(\mathrm{h})\right]$ and the downwelling solar spectral irradiance $\left[\mathrm{E}_{\mathrm{d}}(\mathrm{h})\right]$ is complicated by environmental noise sources including the path radiance $\left(\mathrm{L}_{\mathrm{path}}\right)$, reflected solar glint $\left(L_{S}\right)$, and reflected sky radiance $\left(L_{s k y}\right)$. On fine spatial scales, glint is strongly affected by sea state and wave characteristics.

aquatic applications on above-water fixed platforms and low-altitude aerial drones.

\subsubsection{Radiometrically estimating WQPs from low altitude}

Specific optically-active WQPs of natural aquatic bodies affect the shape and magnitude of the water-leaving radiance spectrum $\left(\mathrm{L}_{\mathrm{w}}(\lambda)\right.$, Fig. $2-2$ in characteristic ways. Chlorophyll is the primary pigment of suspended plant matter (i.e., phytoplankton), and its concentration in aquatic systems is used as a WQP indicating the suspended algal biomass. This pigment absorbs strongly in the blue wavelengths and thus shifts the peak of the water-leaving radiance reflectance spectrum toward the green [Clarke et al., 1970; Morel and Prieur, 1977]. Colored dissolved organic matter (CDOM) in natural aquatic systems absorbs primarily in the ultraviolet, blue, and green wavelengths and thus shifts the reflectance peak toward the yellow Morel and Prieur, 1977; Gholizadeh et al. 2016. Suspended particulate matter (SPM), often inorganic in composition (e.g. silt and sediment) typically has a broad scat- 
tering spectrum which increases the overall magnitude of water-leaving radiances in aquatic systems Kirk, 1994. Algorithms developed to estimate these and other WQPs typically rely on simple band-ratios of different wavelengths with spectral widths of tens of $\mathrm{nm}$ (Fig. 2-1) Hoge et al., 1987. Such simple band-ratios are often adequate for many environmental applications, but reflectance measurements with higher spectral resolution can in principle increase the number of WQPs that can be simultaneously recovered. Operationally, such a hyperspectral approach to estimating multiple WQPs simultaneously has significant advantages in environmental monitoring applications, especially those employing aerial drones with limited flight capabilities. Similarly, hyperspectral data can also simultaneously provide additional wavelengths for applying glint-correction algorithms (Fig. 2-1), which can increase the accuracy of estimated WQPs by minimizing the effect of this particular source of noise. 


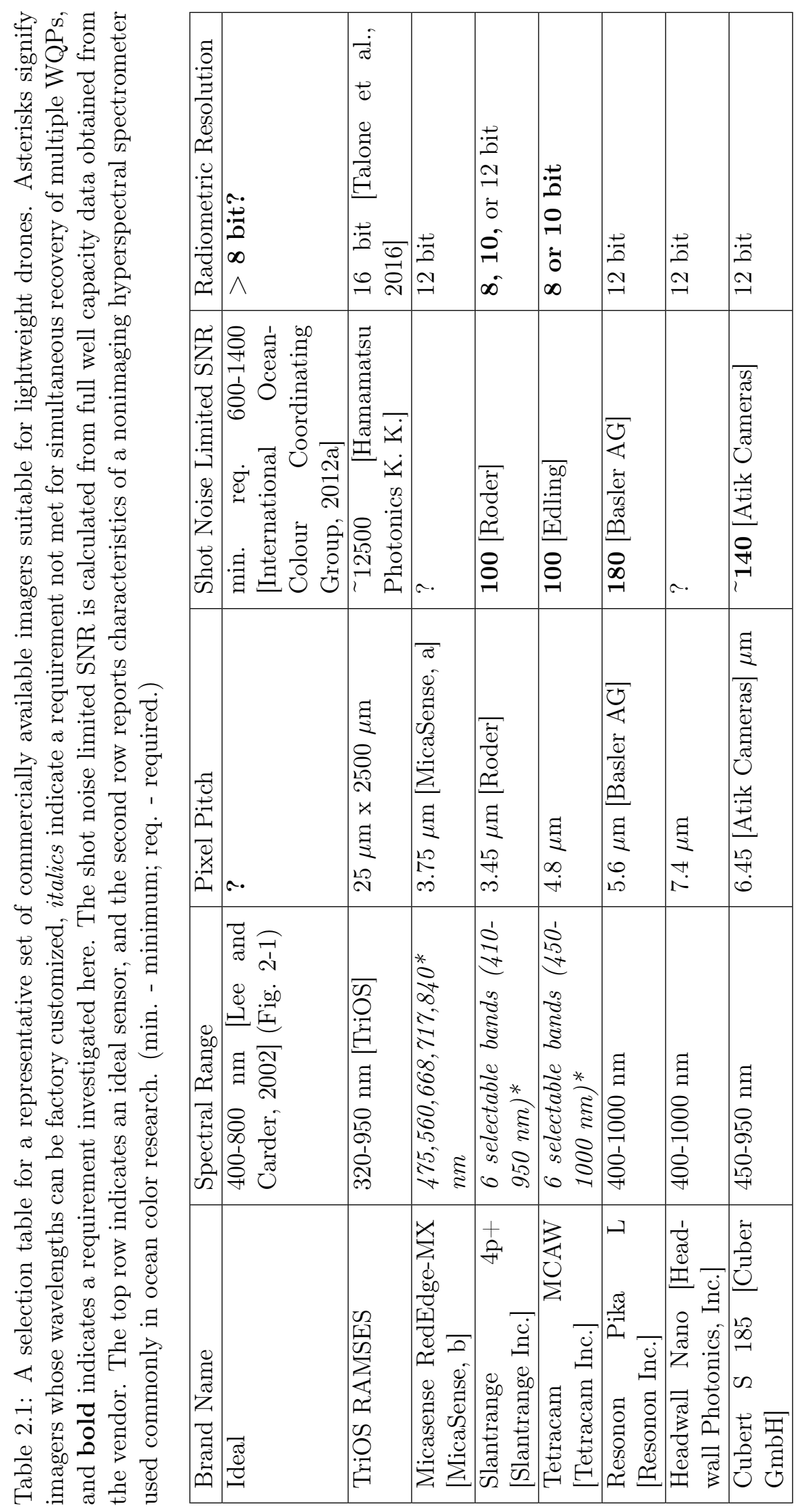


An important caveat is that higher spectral resolution in a radiometric measurement does not necessarily improve the accuracy of the recovered water-leaving radiance signal. For natural aquatic systems roughly 15 spectral bands are considered sufficient to represent the entire hyperspectral range at $5 \mathrm{~nm}$ resolution, due to the spectral interdependence of the water-leaving radiance signal [Lee et al., 2014]. From a representative sample of lightweight off-the-shelf multispectral and hyperspectral imagers currently available in North America (Table 2.1), the multispectral ones do not provide enough discrete wavelengths to approximate hyperspectral data for WQP applications. It is technically feasible to employ multiple individual multispectral imagers in an array in order to achieve this 15-band recommendation, but for drone-based applications such an approach would increase weight and power requirements, which could be prohibitive for use on the 'lightweight' drones as defined by the US Federal Aviation Administration (i.e., $<25$ kgs Federal Aviation Administration (FAA), 2016b|). For practical purposes, hyperspectral imaging is likely optimal for collecting the water-leaving radiance wavelengths needed for applying modern WQP algorithms on fine spatial scales.

\subsubsection{Spectral signal-to-noise ratio considerations}

The theoretical SNR is an estimate of the impact of the inherent noise sources in the imager electronics and light on the measured signal at any pixel. It is defined as the intensity of a radiometric measurement divided by a measure of its noise [Eq. 2.1].

$$
S N R(\lambda)=S(\lambda) / N(\lambda)
$$

Here, $\lambda$ is the wavelength, $S$ is the signal, and $N$ is the noise. For this theoretical per-pixel SNR the signal is taken as the number of electrons on the pixel. By applying a cameraspecific conversion factor, the digital number recorded at any pixel can be converted into this number of electrons. The noise at any pixel $\left(N_{\text {pixel }}\right)$ is a combination of photon shot noise $\left(N_{\text {photon }}\right)$, detector dark noise $\left(N_{\text {dark }}\right)$, readout noise $\left(N_{\text {readout }}\right)$, and digitization noise $\left(N_{\text {digitization }}\right)$ [Eq. 2.2, Hu et al., 2012, Moses et al., 2012a].

$$
N_{\text {pixel }}=\left(N_{\text {photon }}^{2}+N_{\text {dark }}^{2}+N_{\text {readout }}^{2}+N_{\text {digitization }}\right)^{1 / 2}
$$


Photon shot noise $\left(N_{\text {photon }}\right)$ is Poisson distributed, and by assuming high photon levels $N_{\text {photon }}$ can be approximated as the square root of the mean value of electrons recorded at that pixel, after correcting for the detector and readout noise offsets and assuming that the digitization noise is negligible. The dark noise offset can be corrected by subtracting images taken of a dark target at an exposure time that matches the original images exposure time. The read noise offset can be corrected for by taking images of the readout noise at the imager's minimum exposure time of $20 \mu \mathrm{s}$. The digitization noise arises from round-off error when converting from electrons to integer digital numbers and can be assumed negligible due to its theoretically low impact at the typical 12 bit resolution of modern camera systems (Table 2.1). Typically, with high numbers of electrons on a given pixel the SNR can be estimated from the intensity at that pixel by assuming that shot noise dominates.

Several lightweight, low-power, multi- and hyperspectral imagers currently available commercially (Table 2.1) have been used in agricultural applications where small pixel footprint (pitch) is a critical selection requirement, as it provides the highest spatial resolution images. High spatial resolution is especially important in terrestrial applications to eliminate spectral mixing between plant leaves and nearby background terrestrial material (e.g. soil) Zhou et al. 2018. The highest possible shot-noise limited SNR (calculated as the square root of the full well capacity) of the tabulated imagers is 180 (Table 2.1). Although nominally low, this SNR is not limiting for most terrestrial applications, but it may be limiting for ocean color applications which typically require higher sensitivity Moses et al., 2012a; Hu et al. 2012. For satellite ocean color applications the minimum recommended SNR is between 600 and 1400 [International Ocean-Colour Coordinating Group, 2012a]. However, the same level of sensitivity is likely unnecessary for lower altitude applications $(\sim 100$ meters) or above-water platforms because atmospheric path radiance is considerably less important at such low altitudes compared to its integrated effect in top-of-the-atmosphere measurements Kim et al. 2013; Moses et al. 2012a.

In addition to an imager's maximum theoretical SNR, the typical spectral shape of a water-leaving radiance spectrum is also an important consideration in aquatic environmental hyperspectral radiometry. For any given image, only the wavelength with the highest intensity signal on the imager's sensor can reach the maximum possible SNR (limited by the full well capacity). Within the same image, lower intensity wavelengths will not reach the full well capacity and therefore will exhibit a lower SNR. When imaging aquatic scenes, the 
spectral intensity and therefore spectral SNR is determined by the combined spectral shape of the water-leaving radiance signal multiplied by the imager's spectral efficiency (the total spectral efficiency of the system, including the spectral effects of the optics and the quantum efficiency of the detector). The water-leaving radiance spectrum generally exhibits a lower radiance in the NIR relative to visible wavelengths due to the high NIR absorption of water [Pegau et al., 1997]. Depending on the imager optics and electronics, an imager's spectral efficiency can also have a higher efficiency in the visible compared to the NIR and so it can be expected that the NIR will have a lower SNR relative to the visible wavelengths.

One final important consideration when examining imager SNR in such applications is the approach used to correct for glint (Fig. 2-2). Software algorithms have been developed that correct for glint in the visible wavelengths of interest, by using spectral information measured in the NIR Mueller and Austin, 1995; Mobley, 1999; Lee et al., 2010; Lucke et al. 2011. For algorithms presented by Mueller and Austin [Mueller and Austin, 1995 and by Lee et al. Lee et al. 2010, NIR radiances are used to scale the downwelling sky remote sensing reflectance (the radiance divided by the irradiance) spectrum before subtracting it from the total water-leaving remote sensing reflectance spectrum. Due to this scaling, a low signal and by extension low SNR in the NIR may lead to poor glint correction of the visible light. Any errors in correcting measurements in the visible will in turn affect estimates of WQP concentrations, which now depend on both the NIR and band ratios in the visible (Fig. 2-1). Hardware strategies to correct for glint, such as adding a polarizer to the optical train, may also change the spectral SNR and thus affect the accuracy of the WQP estimates.

\subsubsection{Correcting for sun and sky glint within a single image}

Beyond these basic SNR considerations, imaging applications on natural water bodies must also consider within-image variability due to glint and its contribution of variations in reflectance on fine spatial scales. Such effects have been examined by Zeng et al. Zeng et al. 2017 by comparing above-water imagery of an RGB camera to spot measurements made by a nonimaging hyperspectral spectrometer. Glint introduced severe contamination in the spectra of both the RGB camera and hyperspectral spot sensor, with further fine spatial scale glint variations arising from partial cloud cover and surface capillary waves. These fine-scale spatial variations in the reflectance spectra moreover affected the visible and nearinfrared sections of the spectrum, which Zeng et al. [Zeng et al. 2017] noted may lead to 
inaccuracy in chlorophyll concentration estimates once WQP algorithms were applied. A similar study with a drone-based multispectral camera also examined this effect in glintinduced spatial variations [Su, 2017], where areas of a reservoir experiencing wind-driven capillary waves introduced spatial variability in sun and sky reflectances that significantly contaminated estimates of chlorophyll concentration on those fine spatial scales. To take full advantage of the spatiotemporal resolution that low-altitude imaging offers for mapping WQPs in aquatic systems, removal of the glint effects is essential.

Novel image-processing approaches have been explored to reduce the glint induced spatial variation in WQP estimates made from low-altitude drone imagery [Totsuka et al., 2019, Su, 2017], but they each exhibit some limitations. The non-local mean filter used by Totsuka et al. Totsuka et al. 2019] reduces the maximum achievable spatial resolution. Su's $\mathrm{Su}$, 2017] matching pixel by pixel algorithm is only able to remove variations during training of the WQP estimation algorithms by leveraging the in-situ data, so it does not work while estimating WQPs in the field. Two standard algorithm-based glint correction approaches that would preserve pixel-level spatial resolution while potentially improving the WQP estimation accuracy have yet to be assessed quantitatively on fine spatial scales: one proposed by Mueller and Austin [Mueller and Austin, 1995; Mobley, 1999] and another proposed by Lee et al. Lee et al., 2010" (hereafter referred to as MASC (Muller Austin software correction) and SOSC (spectral optimization software correction) respectively).

The MASC approach utilizes NIR subtraction: first subtracting the downwelling sky radiance, scaled by the reflection coefficient calculated for a planar surface at the instruments particular view angle, from the water-leaving radiance signal and then subtracting the value at $750 \mathrm{~nm}$ from the entire spectra [Eq. 2.3, adapted from Mueller and Austin Mueller and Austin, 1995]1.

$$
R_{\mathrm{rs}}(\lambda)=\left\{\left[L_{\mathrm{t}}(\lambda)-\rho * L_{\mathrm{s}}(\lambda)\right]-\left[\left(L_{\mathrm{t}}(750 n m)-\rho * L_{\mathrm{s}}(750 n m)\right]\right\} / E_{\mathrm{d}}(\lambda)\right.
$$

Here $\lambda$ is the wavelength, $L_{t}$ is the total water leaving radiance, $L_{s}$ is the downwelling sky radiance, $\rho$ is the reflection coefficient, and $\mathrm{E}_{\mathrm{d}}$ is the downwelling irradiance. The inherent assumptions in this algorithm are that the contributions at $750 \mathrm{~nm}$ are solely from surface reflected light and that the reflection coefficient can be well estimated by a planar surface (though the spectrally flat subtraction at $750 \mathrm{~nm}$ attempts to correct the inaccurate reflection 
coefficient) Mueller and Austin, 1995, Mobley, 1999. The first assumption is typically true in case I waters, where chlorophyll dominates, but can be false in high sediment load waters, because the sediment exhibits a high reflectance in the NIR. The second assumption, that the reflection coefficient can be well estimated by a planar surface, typically holds at course spatial resolutions where variations due to capillary waves and surface waves are averaged out. However, when imaging on fine scales on the order of centimeters this assumption does not hold Carrizo et al. 2019], gusts of wind and surface waves can change the angle of the wave facets relative to the imager, and decrease the accuracy of the reflection coefficient estimate.

The Lee et al. [Lee et al., 2010] spectral optimization correction (SOSC) algorithm starts by estimating the initial water-leaving radiance signal by first subtracting the reflection coefficient scaled signal in the NIR. This initial estimate is then compared to a modeled water-leaving radiance signal, while an offset value (constant across all wavelengths) is iteratively changed to more accurately match the measured and modeled signals. The modeled water leaving remote sensing reflectance is derived from constant wavelength dependent absorption and backscattering coefficients representing algae, seawater, colored dissolved organic matter, and particles, with associated magnitudes that are also allowed to vary during iteration (except for seawater). The SOSC also assumes that the reflection coefficient is for a planar surface on the initial guess, but the optimization of the offset value to fit the modeled spectra allows for some correction of the absolute offset. Although the SOSC algorithm is able to adapt using the offset value, it still may not perfectly capture the water-leaving radiance signal in all ocean color scenarios, as the correction technique is assuming that the offset value is spectrally flat, where the actual offset (glint) may have a different spectral shape. Additionally, the constant absorption and scattering coefficients used may actually vary by region. Overall, the optimization framework allows for more accurate estimation of the reflected skylight than the MASC algorithm, particularly when sensing in aquatic regions with non-zero NIR water leaving radiances.

Both of these algorithm-based glint correction approaches have been evaluated in field studies, such as Shang et al. Shang et al. 2017 who employed the SOSC approach with a nonimaging ocean color radiometer deployed on a drone platform. However, these approaches have not yet been applied to fine-scale imaging spectrometer data, where certain prior assumptions must be reconsidered. For example, assuming an average reflection co- 
efficient such as one found in both SOSC and MASC will often not match the estimated value based on the view angle [Carrizo et al., 2019], which in principle will decrease the effectiveness of these approaches.

An alternate approach to correct for glint effects in ocean color radiometry involves using a vertical polarizer viewing close to Brewster's angle $\left(\sim 53^{\circ}\right)$, to block the horizontally polarized reflected skylight. Fougnie et al. Fougnie et al., 1999 demonstrated that polarization can be a viable correction technique for glint but Gilerson et al. Gilerson et al. 2018 noted the need for further assessment of this hardware-based approach. Such further assessment is especially appropriate with our intended area of spatial studies of aquatic systems using imaging spectrometers, given that the polarization of glint may vary significantly within the spatial scales of interest due to capillary waves changing the angle at which sun or skylight is reflected off the water's surface.

For the comparison of glint correction approaches presented in this study it was necessary to modify a computational aspect of the SOSC. Specifically, we implemented additional bounds on the optimization approach, in order to minimize the instances where the optimization failed to converge (Appendix C). This change allowed us to obtain a much higher number of realistic fits of model parameters and thus have more data with which to assess the efficacy of the SOSC approach in real-world applications.

\subsection{Materials and Methods}

In this study, the benefits and limitations of using the traditional glint correction approaches (SOSC, MASC, and polarizer) for estimating fine-scale WQPs from imaging spectrometer data are explored in a realistic ocean color setting, from a fixed-platform. The view angle dependence of each glint correction approach is also determined. To implement this study, a radiometrically calibrated representative imaging spectrometer was required.

\subsubsection{A representative lightweight imaging spectrometer}

The Resonon Pika L was chosen as a representative hyperspectral imaging spectrometer whose weight, power, and cost are suitable for fine-scale mapping of aquatic WQPs from either fixed platforms or aerial drones. This system is a line scan imager, obtaining hyperspectral data over a broad spectral range of the visible and near-infrared (400-1000 nm) 
wavelengths, in a single spatial dimension. It is optically fast with an f-number of 2.4. Its shot noise limited SNR (180) is the highest among those we surveyed (Table 2.1). It has 12-bit radiometric resolution, and its software development environment allows for the implementation of auto-exposure algorithms in order to utilize its full dynamic range for each image (though we auto-expose to only $\sim 30 \%$ of the full-well capacity, to allow for capturing data with significant variations due to glint). The ability to auto-expose is an advantage in aquatic remote sensing applications given the large fluctuations that can be expected in incident irradiance, due to varying cloud cover for example. These hardware and software characteristics make this imager a representative example of the current commercially available, lightweight, low-power imaging spectrometers suitable for fine-scale aquatic radiometry.

\subsubsection{Laboratory calibration of imaging spectrometer}

This representative imaging spectrometer was radiometrically calibrated, both with and without a polarizer in the optical train, in the laboratory by using an integrating sphere and a spectroradiometer. The imager captured single images of the interior of the integrating sphere at $20 \mathrm{~ms}$ and $40 \mathrm{~ms}$, while the radiometer measured the radiance internal to the integrating sphere. When applying these calibrations to data later taken in the field, we applied the imaging spectrometer's calibration image (i.e., from the integrating sphere) having the exposure time closest to that used for any given field image (4-87 ms). When the integration times of a field image did not match the intervals used in the laboratory calibration, a linear interpolation was applied, from the nearest calibration image. We also assessed the linearity of the imaging spectrometer using the integrating sphere, and found that it was linear only until the full-well was $\sim 96 \%$ full $(r=0.9999)$, so we do not use near-saturated calibration images. Since only a single radiometric correction image is used for correction, the SNR then becomes limited by the shot noise present in the correction image (as shown in Appendix B. We expect the reduction in SNR due to this single-image calibration approach to be significantly less than the inaccuracies induced by the surface reflected light in the field images. During the assessment of this imager it was also necessary to determine its digital number to electron conversion factor $g$, which was $\sim 7.9$ (Appendix A). 


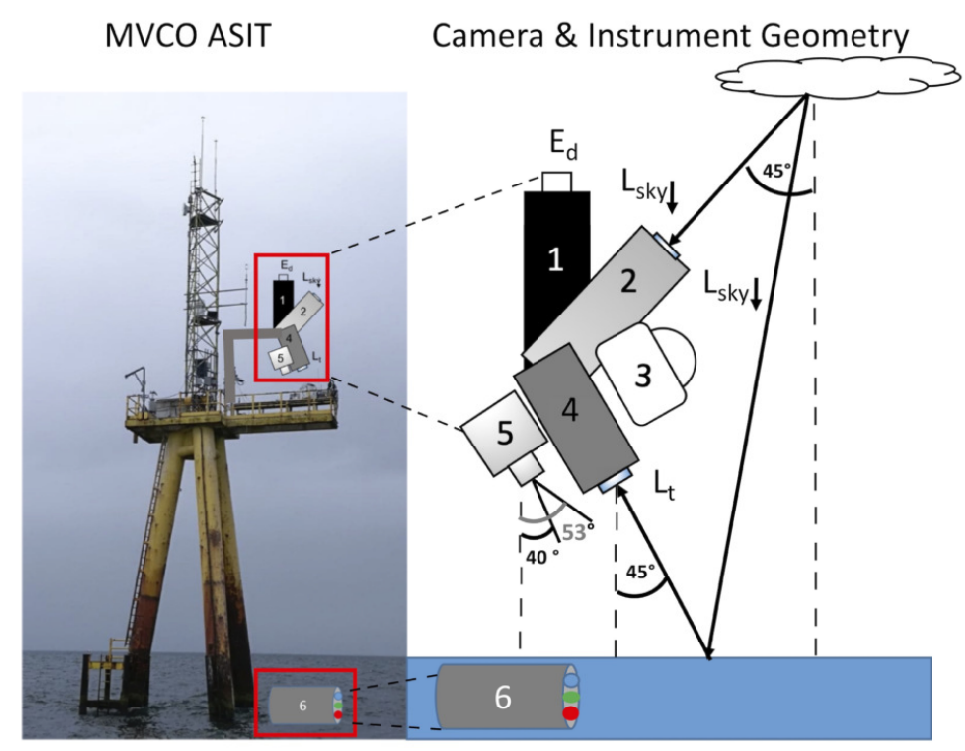

Figure 2-3: The imaging spectrometer and ancillary instruments (right) as deployed at the MVCO ASIT (left). Numbers marking the various components are referenced in Table 2.2

\subsection{Field assessment: fixed-platform study}

We examined the performance of this candidate hyperspectral imager in a 2.5 month field study conducted at the Martha's Vineyard Coastal Observatory Air-Sea Interaction Tower (MVCO ASIT). The MVCO ASIT is located in the coastal Atlantic Ocean, $2 \mathrm{~km}$ south of Martha's Vineyard (Massachusetts, USA) at $41^{\circ} 19.50^{\prime} \mathrm{N}, 70^{\circ} 34.0^{\prime} \mathrm{W}$. This location is strongly tidal with a water depth of $12 \mathrm{~m}$. The height of the platform where the imager and ancillary above-water radiometers were placed (Fig. 2-3 and Table 2.2) is $12 \mathrm{~m}$ above the ocean's surface. 


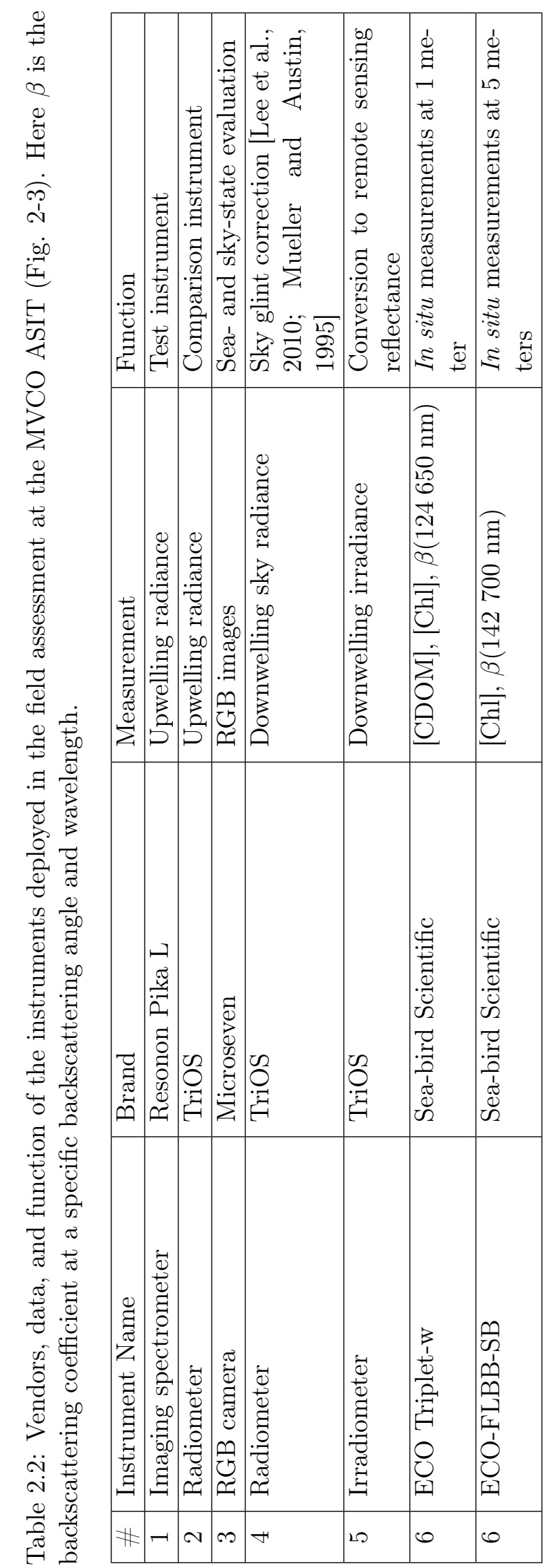




\subsubsection{Imaging spectrometer: observational geometry}

The observational geometry of the imaging spectrometer on the ASIT (Fig. 2-3) was chosen to accommodate basic requirements for the glint correction approaches that are the basis of this study. The spatial axis of the imager was oriented perpendicular to the water surface, viewing from $40^{\circ}$ from nadir (recommended by Mobley [Mobley, 1999] for shipboard measurements) to $53^{\circ}$ (Brewster's angle), where glint from a planar surface would be horizontally polarized [Fougnie et al. 1999]. Further, radiometric data were sampled only when the azimuth angle between the sun and imager was between $115^{\circ}$ and $155^{\circ}$ (encompassing the recommended $135^{\circ}$ |Mobley, 1999|), where glint correction approaches are expected to be tractable.

The observational geometry results in the imager achieving quite fine spatial resolution. The spatial resolution is roughly $3.0 \mathrm{~cm} \times 0.1 \mathrm{~cm}$ over the view angle range, as set by the instantaneous field of view and the spatial geometry. In comparable field applications where such fine spatial resolution is achieved, observations will be more susceptible to changes in the reflection coefficient and polarization state due to capillary waves.

\subsubsection{Ancillary above-water instruments and measurements}

A suite of ancillary instruments were deployed along with the imaging spectrometer to provide observations necessary to evaluate the software sky glint correction approaches, the conversion of radiance to remote sensing reflectance, and estimation of WQPs (Fig. 2-3 and Table 2.2. Downwelling irradiance was measured by an upward-looking irradiometer (1), and sky radiance was measured by an upward-looking radiometer (2) as needed for the SOSC and MASC algorithms. Two RGB cameras (3) imaged the sea state as well as the sky state which provided information on cloud cover. A second spectroradiometer (4) with view angle aligned to the imaging spectrometer (5) provided coincident data for evaluating the radiometric accuracy of the imager (5). The remote sensing reflectance $\left(R_{r s}\right.$, with units of $\mathrm{sr}^{-1}$ ) is then determined as the water leaving radiance at each wavelength as measured by the imaging spectrometer or nonimaging spectroradiometer, divided by the downwelling irradiance at that same wavelength as measured by the upward-looking irradiometer on the ASIT. A servo was used to place a vertical polarizer in front of the imaging spectrometer, under computer control, to obtain both polarized and unpolarized images. 
Environmental conditions including wind speed and incident downwelling solar irradiance were also measured throughout the 2.5 month study. Wind speed was measured by a 3DSonic Anemometer located 3 miles from the ASIT, and ranged from 0.8 to $14 \mathrm{~m} / \mathrm{s}$ during the study (Fig. 2-4, top panel). This large range of wind speeds can be expected to create a variety of surface conditions for testing the glint correction approaches. Data from the above-water downwelling irradiance sensor was used to calculate the incident downwelling solar irradiance, which serves as an indicator of sky conditions (Fig. 2-4, second panel). The incident downwelling solar irradiance varied substantially from day to day in this study, indicating a wide range of sky conditions from overcast to clear skies. Overall these wind speed and sky conditions encompass an environmentally realistic range of non-ideal ocean conditions over which radiometric glint correction approaches must be examined.

\subsubsection{In situ time series of [chl] and $b_{b p 650}$}

Several In situ instruments (Fig. 2-3, '6') were deployed on the ASIT on two subsurface mounts, at $\sim 1 \mathrm{~m}$ and $\sim 5 \mathrm{~m}$, to provide in situ 'truth' observations of chlorophyll concentration ([chl] $)$ and particulate backscattering at $650 \mathrm{~nm}\left(\mathrm{~b}_{\mathrm{bp} 650}\right)$. These time series were used to train and validate our above-water, radiometric WQP estimation algorithms. A WETLabs ECO Triplet-w at $1 \mathrm{~m}$ depth measured the particulate backscattering at $650 \mathrm{~nm}$ and also chlorophyll concentration via a fluorescence approach, exciting at $470 \mathrm{~nm}$ and observing at $695 \mathrm{~nm}$. At $5 \mathrm{~m}$ depth a WETLabs ECO FLBB-SB measured chlorophyll again using the 470/695 excitation/emission pair. The $\mathrm{b}_{\mathrm{bp} 650}$ time series from the $1 \mathrm{~m}$ sensor was sufficient to serve as our in situ truth for its radiometrically estimated analog (Fig. 2-4, third panel), but neither chlorophyll sensor provided data for the entire duration of the time series due to biofouling. To create an in situ truth time series for [chl] we merged the two time series and corrected for daytime fluorescence quenching (Fig. 2-4, bottom panel).

\subsubsection{Algorithms used for WQP estimation: [chl] and $b_{b p 650}$}

The purpose of this study was to examine glint correction approaches suitable for lightweight hyperspectral imagers and not to assess the accuracy of ocean color algorithms that may be applied to these imager data in this specific location. Nonetheless, such an examination still requires use of some basic remote sensing algorithms for chlorophyll concentration and particulate backscatter in order to quantify any improvements that glint correction 

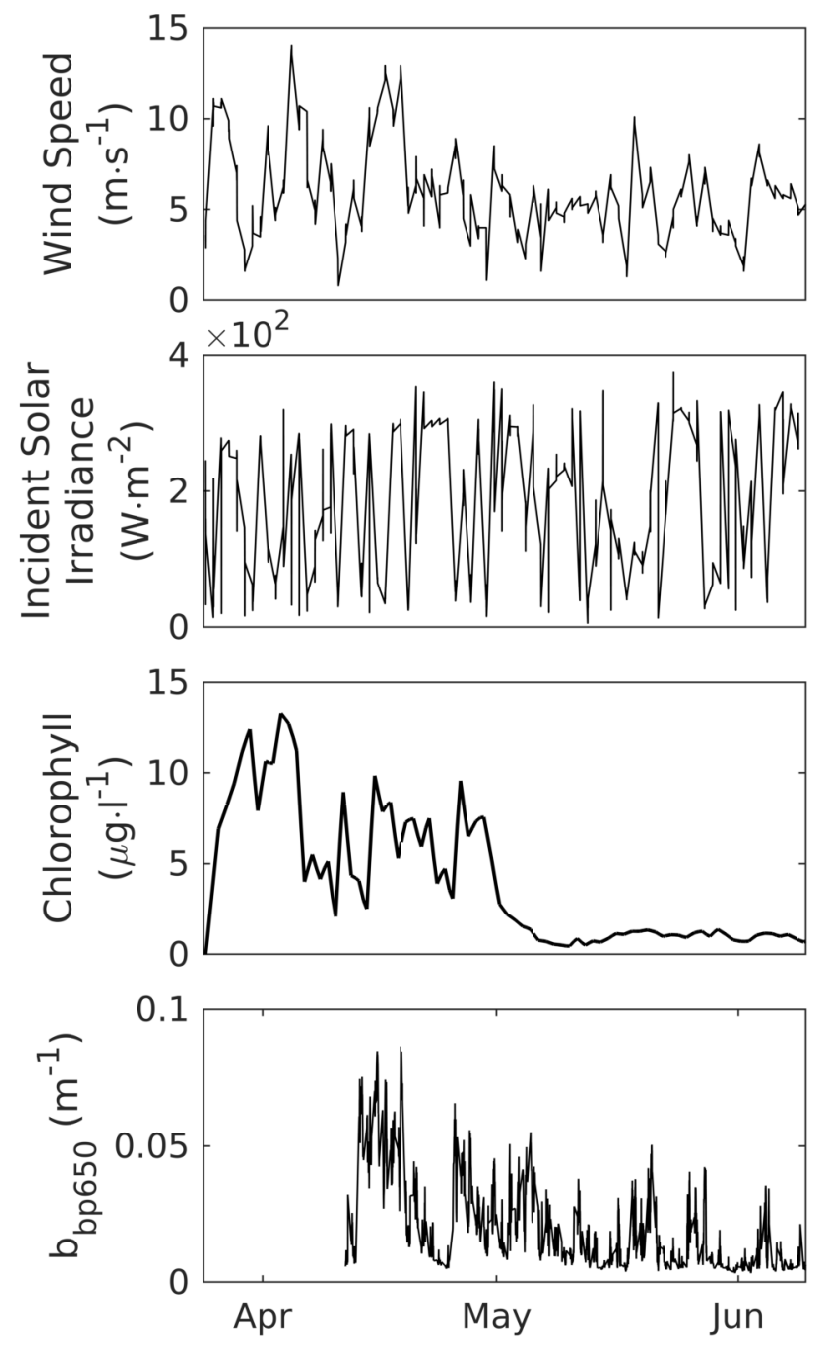

Figure 2-4: Environmental conditions over the 2.5 month field assessment study. Top panel: wind speed. Second panel: incident solar irradiance at the ASIT. Third panel: daily in situ [chl]. Bottom panel: $b_{\mathrm{bp} 650}$.

approaches may have. For this study we modified standard ocean color algorithms to determine chlorophyll concentration and $b_{\mathrm{bp} 650}$ from radiance spectra measured on the imaging spectrometer and the companion nonimaging spectroradiometer. Chlorophyll concentration ([chl]) was estimated using a modification of the Ocean Color 4 (OC4) algorithm [Eq. 2.4],

$$
\log _{10}([c h l])=C_{1} \log _{10}\left(\frac{\max \left(R_{\mathrm{rs}}(443), R_{\mathrm{rs}}(490), R_{\mathrm{rs}}(510)\right)}{R_{\mathrm{rs}}(555)}\right)+C_{0}
$$

where the input $R_{\mathrm{rs}}$ 's are centered at $443 \mathrm{~nm}, 490 \mathrm{~nm}, 510 \mathrm{~nm}$, and $555 \mathrm{~nm}$ and each have $20 \mathrm{~nm}$ bandwidths $\mathrm{O}^{\prime}$ Reilly et al. 1998. For our analyses we used a $1^{\text {st }}$ order polynomial 
instead of the typical $4^{\text {th }}$ order polynomial to avoid overfitting with our narrow data range. Our remote-estimation algorithm for the particulate backscatter coefficient at $650 \mathrm{~nm}$ is adapted from one originally developed for backscatter at $620 \mathrm{~nm}$ [Eq. 2.5 Woźniak et al. 2018.

$$
\log _{10}\left(b_{\mathrm{bp} 650}\right)=C_{2} \log _{10}\left(R_{\mathrm{rs}}(650)\right)^{2}+C_{1} \log _{10}\left(R_{\mathrm{rs}}(650)\right)+C_{0}
$$

This modification allowed us to utilize the $\mathrm{b}_{\mathrm{bp} 650}$ time series that we measure in situ. This algorithm uses a single spectral band centered at $650 \mathrm{~nm}$ with a bandwidth of $20 \mathrm{~nm}$. Using this $1^{\text {st }}$ order polynomial modification of the standard OC4 algorithm, and adjusting the center wavelength of the backscatter algorithm, allowed us to more robustly employ Eqs. (2.4) and 2.5 with our observational data.

To determine the coefficients $\left(\mathrm{C}_{\mathrm{n}}\right)$ of Eqs. 2.4 and 2.5) we split the entire radiometric and in situ data set into individual days and then randomly assigned $\sim 50 \%$ and $\sim 40 \%$ of these days to serve as the training set for coefficient estimation for these two equations. The remaining days were reserved for the validation data set. We then calculated the coefficients for Eqs. 2.4 and (2.5) at each spatial pixel of the imaging spectrometer (i.e., at every view angle) by fitting a polynomial curve to the band ratio and in situ measurements from the assigned training days. Coefficients were calculated at each pixel (view angle) in order to determine the optimal coefficients for each view angle, to examine expected differences in that respect. With these optimizations, any remote sensing reflectances used in the WQP estimation algorithms that exceeded $0.2 \mathrm{sr}^{-1}$ or were below $0 \mathrm{sr}^{-1}$ indicate physically unrealistic data and were therefore omitted from coefficient training and WQP accuracy validation. Finally, we calculated these two WQPs from the validation days using the coefficients. Any predicted values an order of magnitude higher or lower than the maximum or minimum seen in the training set were also excluded.

Because our field study only spanned $\sim 2.5$ months, the specific days selected to train the coefficients $\left(\mathrm{C}_{\mathrm{n}}\right)$ of Eqs. 2.4 and 2.5 could introduce artifacts into the accuracy of these two WQPs and their apparent dependence on view angle. To minimize this potential artifact we performed the coefficient training and subsequent WQP estimation process for 30 separate randomized realizations, and then calculated the mean absolute percent error (MAPE) from these validation sets at each view angle. We then smoothed the results using a median filter over the 30 realizations to better observe underlying trends in the 
relationship between MAPE and view angle. This relationship was largely insensitive to the coefficients derived from any individual training set. This strategy increased confidence in any subsequent analyses despite having only a relatively short data set. One further smoothing step was used to median-filter these MAPE values in an angular sense, using a window of $1.5^{\circ}$ width over the range of view angles observed by the imaging spectrometer $\left(39.75-53.25^{\circ}\right)$. This decreased a large apparent pixel-to-pixel variation in the median MAPE in estimates of $b_{\mathrm{bp} 650}$ that were obtained from the algorithm-corrected observations.

\subsection{5 $R_{\mathrm{rs}}$ Quality Assurance}

An alternative metric for comparing the glint corrected $R_{r s}$ 's measured in the field study, which does not depend upon the WQPs and does not require in situ radiometric measurements, is the quality assurance (QA) as defined by Wei et al. Wei et al., 2016. The QA for an individual spectrum is a measure of how well the $R_{r s}$ matches with previously observed ocean color spectra. The QA is typically calculated from 9 wavelengths spanning $412 \mathrm{~nm}$ to $680 \mathrm{~nm}$. First the $R_{\mathrm{rs}}$ is assigned to an ocean color group, next the $\mathrm{R}_{\mathrm{rs}}$ of each wavelength is checked against bounds set from prior information. If the $\mathrm{R}_{\mathrm{rs}}$ is within the bounds for that wavelength it receives a 1 , if it is outside the bound it receives a 0 . The results are summed and normalized to 1, with 0 denoting a low QA and 1 denoting a high QA.

\subsection{Results}

\subsubsection{Glint correction approaches and radiometric precision}

The 2.5 month field study provided environmentally representative radiometric data for examining the performance of this representative imaging spectrometer over varied insolation conditions, wind speeds, and magnitudes of each WQP. We computed the SNR using selected images from this observational data set (Introduction section 2.1.3 and Appendix B. Images used for this purpose were chosen specifically to span bright and dark insolation conditions as well as high and low concentrations of chlorophyll. The water-leaving radiance and the SNR are highest in the blue-green wavelengths and lowest in the short-blue, red, and NIR wavelengths (Fig. 2-5). The SNR exhibits a shape similar to the radiance because the number of electrons captured in a given exposure time is proportional to the radiance. However, spectral SNR is not perfectly proportional to the radiance across these 

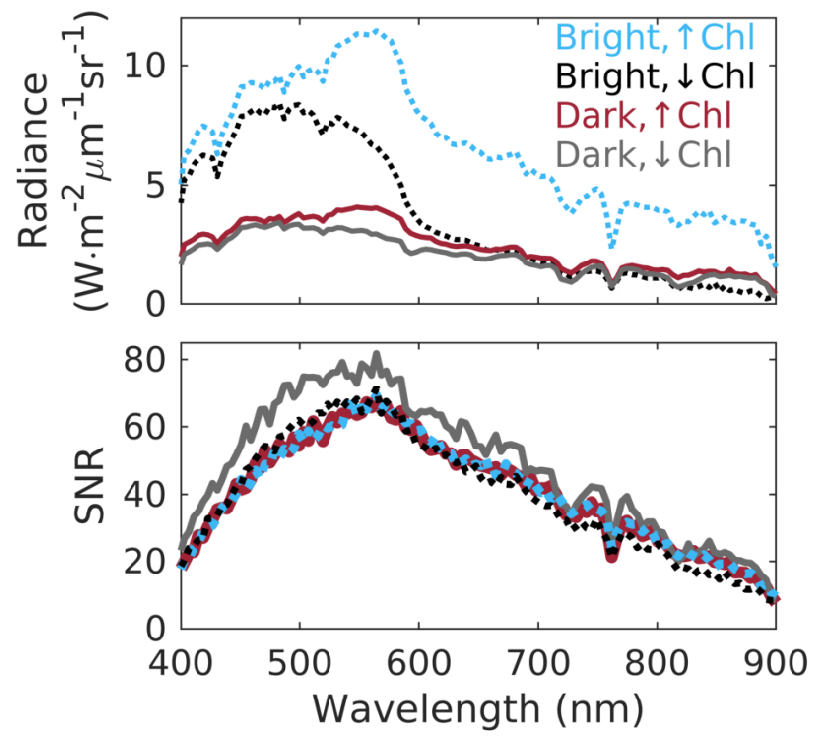

Figure 2-5: Top panel: Water-leaving radiances during high [chl] conditions were maximum at $\sim 550 \mathrm{~nm}$ in this field study whereas maxima were $\sim 490 \mathrm{~nm}$ during low [chl] conditions. Conditions of high downwelling irradiance (i.e., bright sunlight) corresponded with greater upwelling radiances. The shape of the SNR is comparable to its associated radiance, with higher SNRs lining up with higher relative intensities.

wavelengths because of the camera's spectral efficiency and the single image radiometric correction approach we employed (explained in Materials and Methods section 2.2.2 and Appendix B . Since each image was auto-exposed to capture a specific amount of electrons in the blue-green section of the spectrum, the maximum SNR values for each image are independent of large downwelling irradiance fluctuations: the same number of electrons are captured in the blue-green section of the spectrum under bright and dark illumination. The median SNR over the entire time series shows the same characteristic SNR shape, with highest values in the green and lower values in the red and NIR for both the uncorrected and polarizer corrected data (Fig. 2-6). 'Uncorrected' data represent radiometrically-corrected measurements with no glint correction applied. Overall, the SNR of these field-observed images is highest in the blue-green and lowest in the dark blue and far-red to NIR, despite large differences in insolation and WQP magnitude seen throughout the field assessment.

Since these correction approaches remove glint from the total measured radiance, they decrease the radiance, and in turn the estimated $R_{\mathrm{rs}}$, nonuniformly across this spectral range relative to the uncorrected ' $R_{\mathrm{rs}}$ ' (Fig. 2-7, top panel). The uncorrected ' $\mathrm{R}_{\mathrm{rs}}$ ' is calculated using the total sensor measured radiance in place of the water leaving radiance. The ap- 


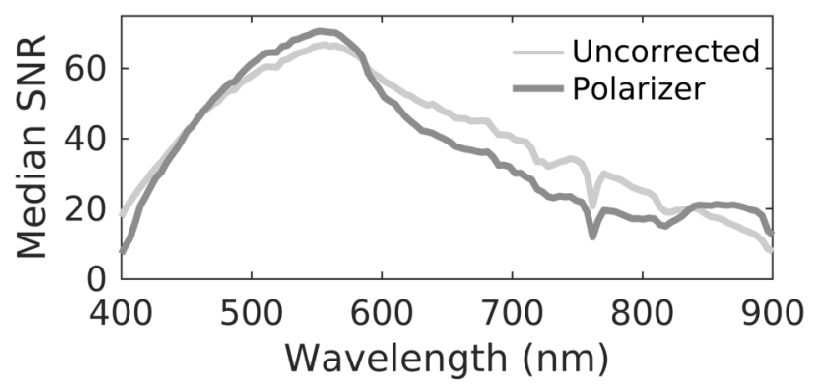

Figure 2-6: The median (theoretically-derived) SNR as calculated over the entire time series. Highest SNRs are in the green section of the spectrum for both spectra. Use of a polarizer decreased the SNR in the red and part of the NIR. The uncorrected SNR data represent a range of view angles between $39.75-42.70^{\circ}$ and the polarizer-corrected SNR is calculated from a range of view angles from $50.30-53.25^{\circ}$.

proaches also decrease the standard deviation of $\mathrm{R}_{\mathrm{rs}}$, here calculated around $39.75-42.70^{\circ}$ for uncorrected, SOSC, and MASC $\mathrm{R}_{\mathrm{rs}}$ and $50.30-53.25^{\circ}$ for polarizer corrected $\mathrm{R}_{\mathrm{rs}}$, towards their theoretical limits (Fig. 2-7, bottom panel). These theoretical limits in $\mathrm{R}_{\mathrm{rs}}$ are determined by the theoretical SNR and were computed by dividing the median $\mathrm{R}_{\mathrm{rs}}$ by the median spectral SNR (Fig. 2-6). Observations corrected using the SOSC and polarizer approaches exhibited the lowest spectral standard deviations (i.e., noise) at these view angles and across this spectral range, while data glint-corrected using the MASC algorithm showed only a slightly lower standard deviation compared to the uncorrected data (Fig. 2-7. bottom).

\subsubsection{Glint correction approaches and WQP estimate accuracy}

The results shown in Fig. 2-7 indicate that the SOSC approach can achieve the highest precision because it exhibits the lowest standard deviation of measured $R_{r s}$. By applying the SOSC glint correction approach and using $\mathrm{R}_{\mathrm{rs}}$ data observed at $40^{\circ}$, the resulting radiometrically-estimated values for both [chl] and $b_{b p 650}$ showed strong correlation with the in situ measured values across a large but environmentally realistic dynamic range (Fig. 2-8). Correlation coefficients for [chl] and $b_{\mathrm{bp} 650}$ using the SOSC approach were 0.68 and 0.73 respectively (Model II geometric mean linear regression, [Peltzer, 2016]), which is a substantial improvement over the correlation coefficients of the uncorrected data $(0.65$ and 0.55 respectively).

To demonstrate the impact of the capillary waves on the uncorrected ' $R_{\mathrm{rs}}$ ', the $R_{\mathrm{rs}}$ and 


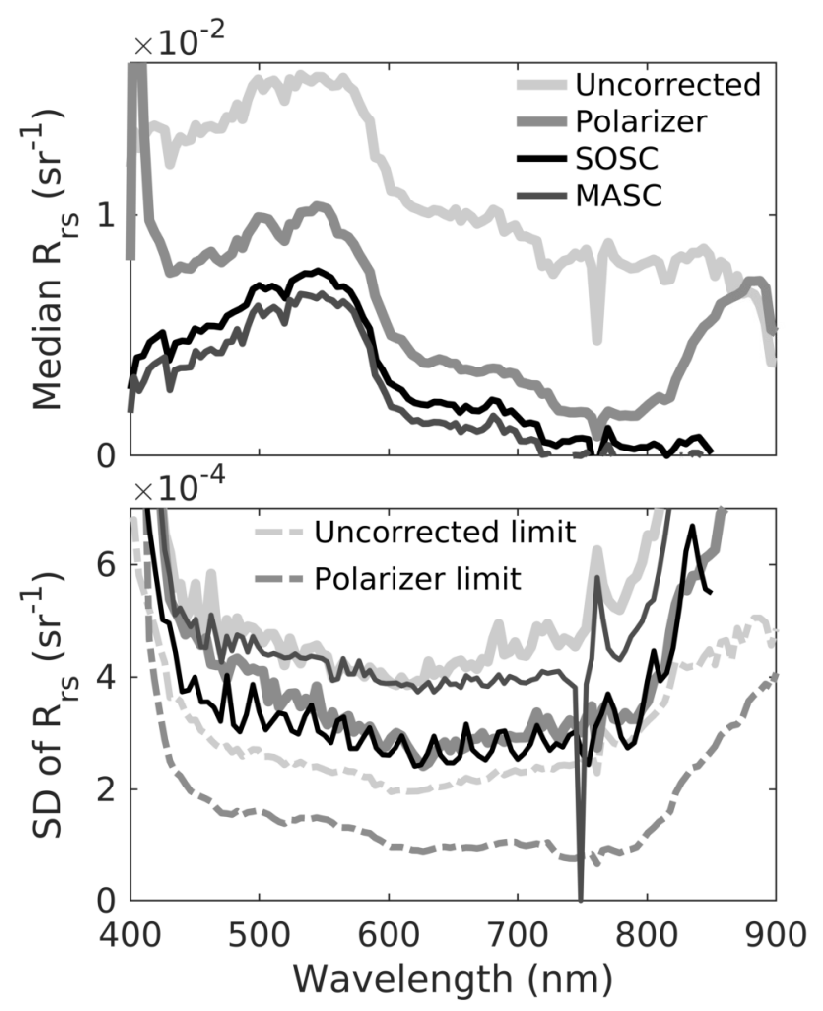

Figure 2-7: The median $\mathrm{R}_{\mathrm{rs}}$ and standard deviation for the entire time series, as a function of wavelength. Each glint correction approach reduces the median $R_{r s}$ (top panel) and decreases the SD towards the shot noise limited theoretical value (bottom panel) relative to the uncorrected ' $\mathrm{R}_{\mathrm{rs}}$ ' (in which the total radiance approximates the water leaving radiance).

the associated chlorophyll estimates are plotted against distance starting from the lowest view angle (where the higher distances are the higher view angles of the imager) (Fig. 2-9. top panel). The view angle range covers a short spatial range $(\sim 6 \mathrm{~m})$ that we assume is homogeneous with regards to its WQPs. The capillary waves (bright vertical lines in the uncorrected dataset) reduce the precision of the chlorophyll estimates made from the uncorrected $\mathrm{R}_{\mathrm{rs}}$ (Fig. 2-9, top panel). The SOSC approach attempts to correct for the capillary waves, and in this example case increases the precision of the chlorophyll estimates (Fig. 2-9, bottom panel). Although the SOSC algorithm increases the precision in particularly wavy waters (shown here), on calm days the chlorophyll estimate from the uncorrected data can be more precise (not shown).

Further analysis revealed that all three of the glint correction approaches led to decreases in the MAPE of estimates of [chl], and thus increases in its accuracy, but with varying effects among each approach (Fig. 2-10, top panel). In terms of their MAPEs the SOSC 


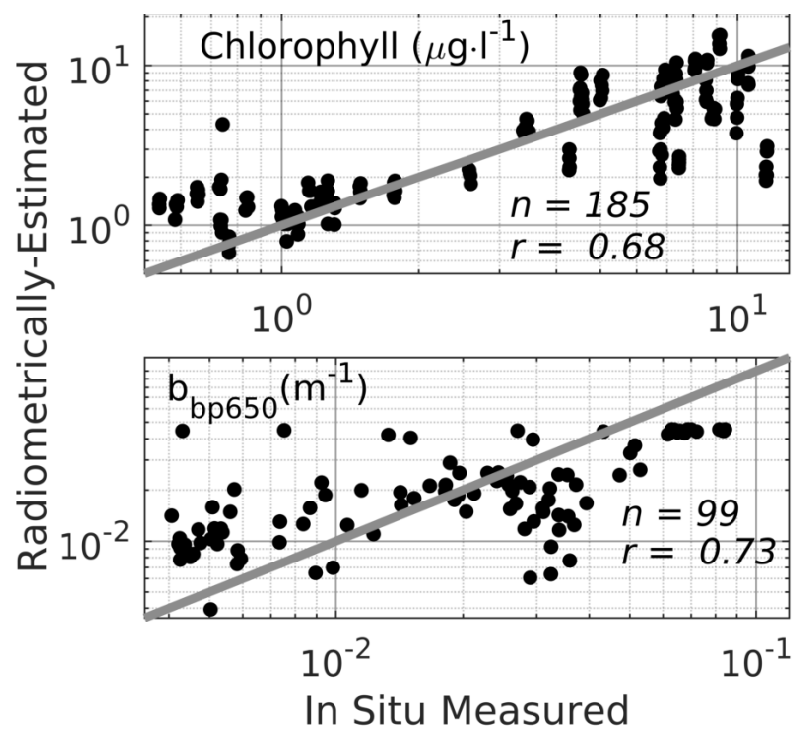

Figure 2-8: Training data relationships between in situ measured (abscissa) and radiometrically-estimated (ordinate) WQPs of chlorophyll (top panel) and $b_{\mathrm{bp} 650}$ (bottom panel) using Eqs. 2.4 and 2.5 respectively. The SOSC glint correction approach was used in both cases. Grey lines define a 1:1 relationship.

approach provided the best radiometric estimates of [chl], followed by MASC, followed by the hardware solution of using a polarizer. For $b_{\mathrm{bp} 650}$ however, MAPE decreased in only two of the three approaches: when the SOSC approach was applied and in certain cases when employing the polarizer, depending on the view angle. The MASC approach provided the least accurate estimates of $b_{\mathrm{bp} 650}$ and there was no uniform difference between the uncorrected and polarizer-corrected data across these view angles. For both of these WQPs the MAPE of the best-case estimates (i.e., those utilizing SOSC glint correction) were relatively insensitive to view angle over the $40-50^{\circ}$ range, but became increasingly sensitive at angles above $50^{\circ}$ (Fig. 2-10). When utilizing the MASC approach, MAPE was lowest near $40^{\circ}$ and increased as the view angle neared $53^{\circ}$. The MASC approach more frequently failed to produce reasonable results than the other two glint correction approaches when estimating $\mathrm{b}_{\mathrm{bp} 650}$ due to several factors, notably by substantially over- or under-correcting for the glint. This over- and under-correction leads either to physically unrealistic magnitudes of $\mathrm{R}_{\mathrm{rs}}$ (e.g., $<0$ or $>0.2 \mathrm{sr}^{-1}$ ) or WQP estimates that fall beyond an order of magnitude of those in the training set. Under such conditions the MASC approach introduces erroneous results for a large fraction of the input data, from $\sim 3 \%$ of observations made at a $40^{\circ}$ view angle to as high as $\sim 24 \%$ of observations made near a $53^{\circ}$ view angle. Rejection rates for poor fits 


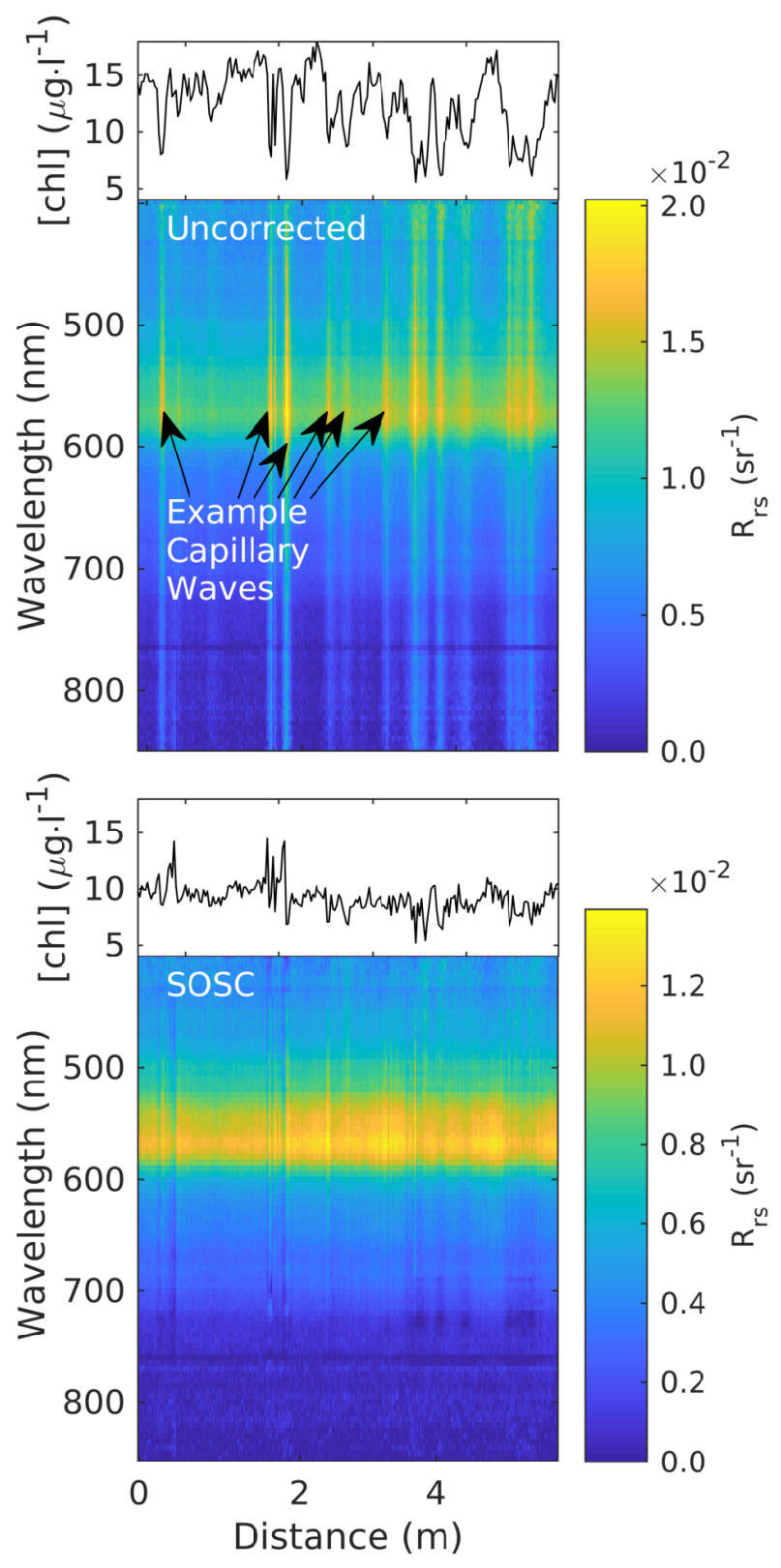

Figure 2-9: An example of hyperspectral $R_{r s}$ data taken with the candidate imager first uncorrected (top panel) and after applying the SOSC approach (bottom panel). An example spatial distribution of remotely estimated [chl] that would be interpreted from these data is presented at the top of each panel. Artifacts due to glint can clearly be seen as bright streaks across all wavelengths at specific distances in the uncorrected images (arrows). Left uncorrected this introduces substantial spatial variability in the estimated [chl] that is solely an artifact of variations in the glint. Here, the SOSC approach reduces this artifact considerably. The in situ [chl] is $8.6 \mu \mathrm{g} \cdot \mathrm{l}^{-1}$. 
when using the other two glint correction approaches are largely insensitive to view angle, remaining below $5 \%$ of the data set. For data glint-corrected with the polarizer, the MAPE of both WQPs was highest near a $40^{\circ}$ view angle and lowest from $44^{\circ}$ to Brewster's angle at $53^{\circ}$ (Fig. 2-10). The MAPE of input images with no applied glint correction (uncorrected) was lowest for [chl] observed around $40^{\circ}$ and highest around $53^{\circ}$ (Fig. 2-10, top panel). For $b_{b p 650}$ the MAPE was highest near $40^{\circ}$ and lowest when approaching $53^{\circ}$ (Fig. 2-10. middle panel). These results show that for each glint correction approach there is a similar, dependence of MAPE on view angle for radiometrically-derived estimates of these WQPs.

The mean QA of each correction method across the time series, median filtered across view angle with the same filter size as the MAPE of the WQP estimates in Fig. 2-10, exhibited significant negative correlation with the view angle dependence of the MAPE in the estimates of the two WQPs. The QA was calculated from radiances at only 8 wavelengths, omitting $412 \mathrm{~nm}$, as the polarizer corrected data was anomalous at this lowest wavelength (Fig. 2-7, top panel). The correlation coefficients between the view angle dependent mean QA (Fig. 2-10, bottom panel) and median MAPE for [chl] (Fig. 2-10, top panel) were -0.98, $-0.73,-0.80$, and -0.89 and for the uncorrected, polarizer, MASC, and SOSC glint removal methods respectively (Model II geometric mean linear regression, Peltzer, 2016). For b bp650 the correlation coefficients calculated between the QA and MAPE were 0.50, $-0.72,-0.89$, and -0.82 for the uncorrected, polarizer, MASC, and SOSC glint removal methods respectively. The relative order of the correction methods, as measured by the maximum QA within the view angle range, in descending order was the SOSC (0.91), polarizer (0.83), MASC (0.81), and then uncorrected data (0.63). The correlation coefficients demonstrate that the view angle dependence of the MAPE in the estimates of both WQPs are significantly negatively correlated with the $\mathrm{QA}$ of the $\mathrm{R}_{\mathrm{rs}}$, except in the case of $\mathrm{b}_{\mathrm{bp} 650}$ estimation from uncorrected data.

The nonimaging spectroradiometer deployed in parallel on the ASIT (Fig. 2-3, label '4') provided data for comparing the radiances measured by this imaging spectrometer against a state-of-the-art ocean color field instrument. When no glint correction is applied to either the imaging and nonimaging hyperspectral data, the MAPEs of both WQPs are in close agreement (Fig. 2-10, light circles, top and middle panels). When glint corrections are applied, however, differences can be seen. When the SOSC approach is applied, the MAPEs from the imager and the nonimaging radiometer agree for estimates of [chl] (top panel) but 

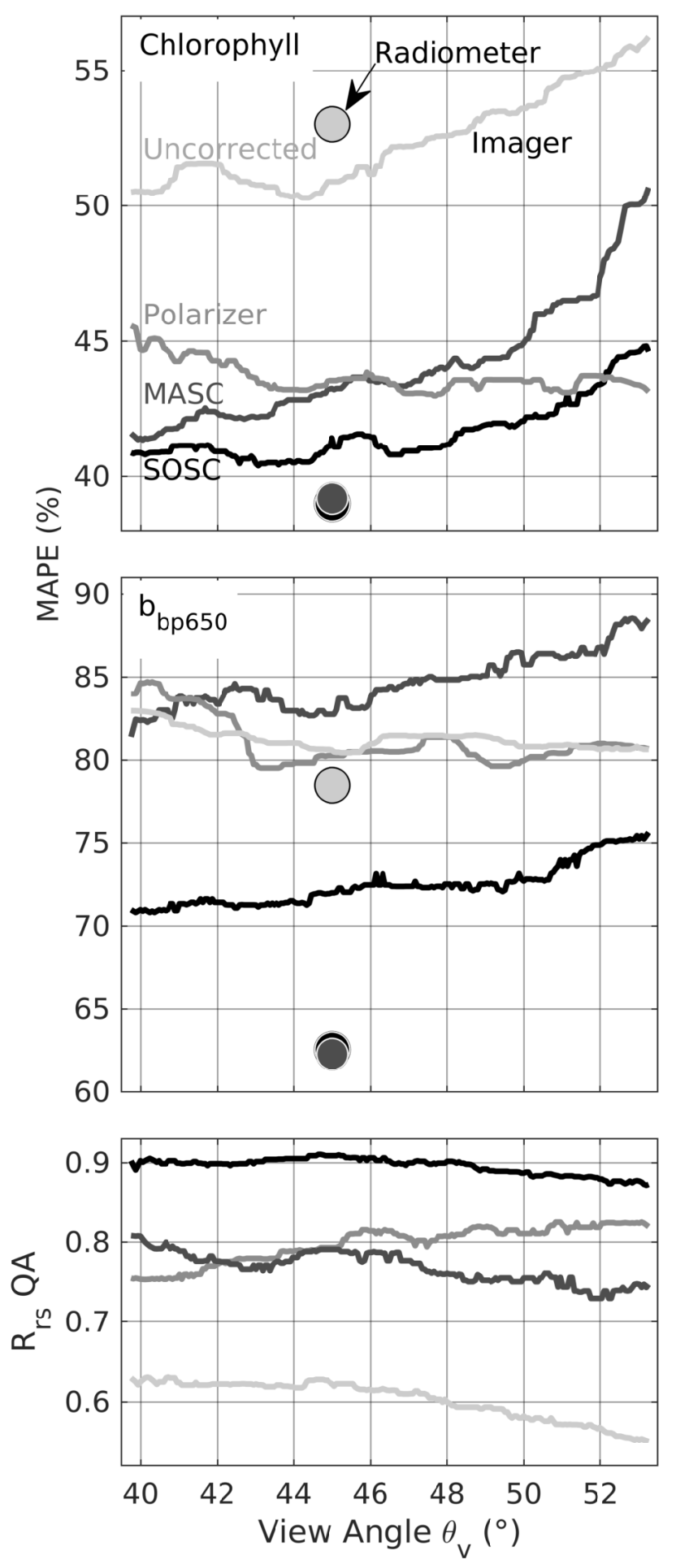

Figure 2-10: The median MAPE between estimates of [chl] and $b_{b p 650}$ as determined from radiometric algorithms. Measurements from a nonimaging hyperspectral radiometer (circles) were made at only one view angle $\left(45^{\circ}\right)$ whereas the vertical orientation of this imaging spectrometer in our deployment allowed us to examine MAPE along a broader range of view angles. Notations in the top panel indicate which trace relates to each glint correction algorithm. The mean Wei et al. Wei et al. 2016 quality assurance (QA) of the $\mathrm{R}_{\mathrm{rs}}$ generally demonstrates an inverse relationship with the MAPE of the WQP estimates. 
Table 2.3: Minimum median MAPE in estimates of [chl] (top rows) and b bp650 (bottom rows), derived from radiometer-measured and imager-measured spectral radiances, comparing three glint correction approaches (polarizer, SOSC, and MASC) and the non glint corrected (uncorrected) data. The radiometer's view angle (relative to nadir) was $45^{\circ}$ and the imagers was the optimal view angle that minimized MAPE.

\begin{tabular}{ccccc}
\hline$[$ chl $]$ & Uncorrected & Polarizer & SOSC & MASC \\
\hline Radiometer & $53.0 \%$ & $\mathrm{~N} / \mathrm{A}$ & $39.0 \%$ & $39.2 \%$ \\
Imager & $48.7 \%$ & $41.8 \%$ & $38.5 \%$ & $39.6 \%$ \\
(View angle) & $43.7^{\circ}$ & $44.3^{\circ}$ & $44.6^{\circ}$ & $40.6^{\circ}$ \\
\hline $\mathrm{b}_{\text {bp650 }}$ & Uncorrected & Polarizer & $\mathrm{SOSC}$ & $\mathrm{MASC}$ \\
\hline Radiometer & $78.5 \%$ & $\mathrm{~N} / \mathrm{A}$ & $62.6 \%$ & $62.3 \%$ \\
Imager & $79.0 \%$ & $77.5 \%$ & $67.3 \%$ & $75.5 \%$ \\
(View angle) & $45.4^{\circ}$ & $46.3^{\circ}$ & $42.8^{\circ}$ & $41.3^{\circ}$ \\
\hline
\end{tabular}

not for estimates of $b_{b p 650}$ (bottom panel). When applying the MASC approach, neither of the two WQPs showed agreement between the imaging and nonimaging spectrometers. These results indicate that the representative imager can achieve a MAPE for [chl] roughly within $5 \%$ of that obtained with a nonimaging spot radiometer with all glint correction approaches. In contrast, although the SOSC approach provided a clear advantage over the other two glint correction approaches for $b_{\mathrm{bp} 650}$ made with the imager, the spot radiometer nonetheless achieved roughly $10 \%$ better MAPE for this WQP.

These results can be summarized in terms of the median MAPE between the radiometricallyestimated WQPs and their in situ measured counterparts, for each glint correction approach at its lowest MAPE view angle (Table 2.3). It is important to note that the exact accuracies may not be visible in Fig. 2-10 due to the median filtering over the view angle. Overall, the imaging spectrometer and radiometer were similarly accurate in estimates of [chl]. All three approaches achieve similar accuracy in [chl], improving estimates by roughly 7 to $10 \%$. For $b_{b p 650}$ the imager has a lower accuracy than the spot radiometer for all three glint correction approaches, most dramatically by over $13 \%$ MAPE for estimates of $b_{b p 650}$ when the MASC approach is applied. The SOSC approach provides the highest accuracy in imager-derived estimates of $b_{b p 650}$. Overall, the hyperspectral imager achieves similar estimation accuracy as the spot radiometer for the uncorrected data, and the SOSC approach achieves the highest accuracy estimates when using the hyperspectral imager. 


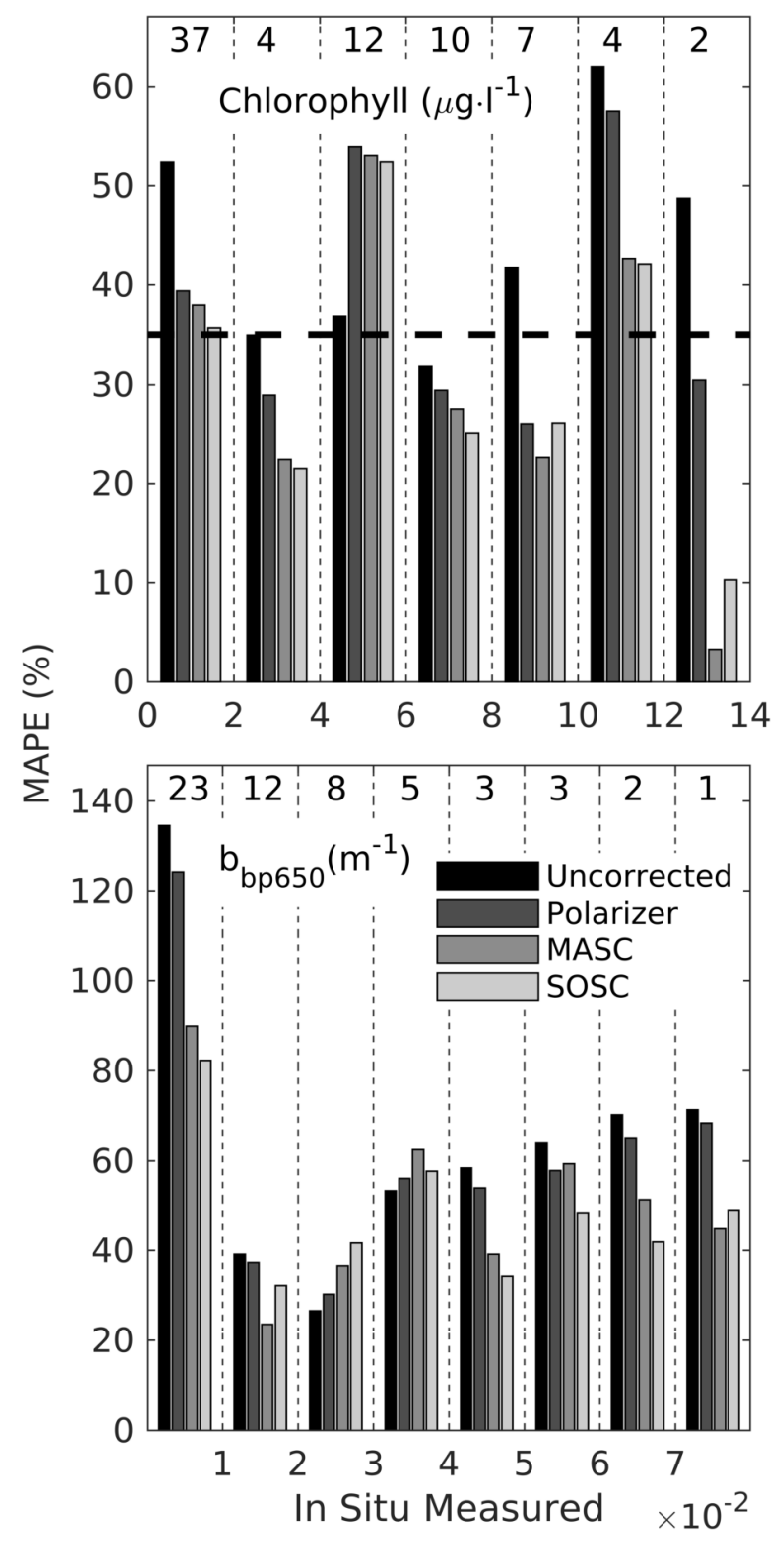

Figure 2-11: The MAPE of median daily estimates of [chl] and $b_{b p 650}$, binned across the dynamic range observed in the field study. Numbers above each bin indicate the median number of data points used. The dashed line indicates the $35 \%$ [chl] accuracy goal for the SeaWiFS satellite sensor [McClain et al. 1998.

With the optimal view angles now determined for each glint correction approach, it is possible to examine variability in MAPE for each WQP across the dynamic ranges seen in the field study (Fig. 2-11). Glint-corrected [chl] and b bp650 estimates typically exhibited lower MAPE at the high and low ends of these distributions whereas glint correction actually 
increased MAPE in the middle of these ranges $\left(4-6 \mu \mathrm{g} / \mathrm{l}\right.$ and $0.02-0.04 \mathrm{~m}^{-1}$ for [chl] and $\mathrm{b}_{\mathrm{bp} 650}$ respectively). A caveat with the low MAPEs seen at in the high value range of both WQPs is that these ranges corresponded with fewer observations, which may affect these results. For [chl], these glint correction approaches improve accuracy towards the 35\% ideal goal for the SeaWiFS satellite sensor [McClain et al., 1998]. Overall, this analysis demonstrated that the ranking of accuracy in these three glint correction approaches holds across the entire dynamic range of WQP values observed in this field study, with the SOSC approach generally being preferable.

\subsection{Discussion}

This study is one of the first to compare algorithm-based and hardware-based glint correction approaches to fine-scale WQP assessment scenarios that employ an imaging spectrometer. This study examined a line-scan hyperspectral imager but these findings are equally applicable to imagers that capture two-dimensional spatial images with an added spectral dimension. These results have broader implications for selecting and deploying glint correction approaches for field studies, but several aspects of this study merit additional discussion.

\subsubsection{Glint correction in a fine-scale ocean imaging context}

The spectral signal-to-noise ratio of this particular imaging spectrometer did not limit its accuracy in estimates of [chl] and $b_{\mathrm{bp} 650}$ when no glint correction was applied. Despite the fact that the per-pixel SNR of the imaging spectrometer (Fig. 2-6) was lower than what is prescribed for satellite ocean color applications [Mueller et al., 2003], it was still sufficient to match the accuracy of the non-imaging spectrometer using the uncorrected data (Fig. 2-10). Therefore, it may be possible to collect even fewer photons while still maintaining the estimation accuracy with these empirical algorithms, which would allow for finer spatial or spectral resolution for cameras mounted on moving platforms. However, it would be ill-advised to not implement a glint correction approach, as they generally granted higher [chl] estimation accuracy.

In contrast to the uncorrected data, when correcting for glint the combination of low spectral SNR of the imager and fine-scale variations in the surface-reflected light limited the WQP estimation accuracy. This limitation occurred when either the MASC or the 
SOSC approaches were used to remove glint before estimating $b_{\mathrm{bp} 650}$ using this imaging spectrometer (Fig. 2-10, middle panel). It follows that the estimates of $b_{b p 650}$ would be more sensitive to the glint correction uncertainties, as the uncertainty represents a larger percentage of the $R_{\mathrm{rs}}$ at $650 \mathrm{~nm}$ than in the blue-green wavelengths (Fig. 2-7). It is also possible that the error in the MASC algorithm arises due to over-correction when $b_{\mathrm{b} 650}$ is at elevated values, due to the algorithms assumption that the NIR light is solely due to glint. Modeling approaches could potentially provide a means to identify if the inaccuracy is due to the low SNR in the NIR, the increased variability resulting from variations in the surface-reflected light at these fine spatial scales, or other assumptions made by each algorithm.

Although our comparison of glint correction approaches were only applied to two particular WQPs ([chl] and $\left.\mathrm{b}_{\mathrm{bp} 650}\right)$, the radiometric precision gained by applying glint correction approaches applies across the imager's entire spectral range and thus will benefit estimates of other WQPs that rely on wavelengths different than the ones we used to estimate [chl] and $b_{\mathrm{bp} 650}$ in this study. All three corrective approaches reduced the absolute variability in the $R_{r s}$ below the precision achieved in the uncorrected data (Fig. 2-7, bottom panel). Yet none of these three approaches eliminated all of the fine-scale spatial variability because they were unable to reach the theoretically-derived precision set by shot noise (Fig. 2-7. bottom panel). Any remaining imprecision is likely due to imperfect correction of the finescale glint variations, but it could theoretically also reflect actual fine-scale environmental spatial differences in the water-leaving radiance such as that generated by spatial patchiness in suspended particulate matter.

Even though the SOSC approach increased the precision of [chl] estimates despite capillary waves as in Fig. 2-10, not every scenario shows an increase in the precision relative to the uncorrected data. Although the SOSC approach typically removes the offset in the $\mathrm{R}_{\mathrm{rs}}$ due to capillary waves, this did not lead to increased precision in the [chl] estimates derived from every image. Oddly, the SOSC approach often increased the imprecision on particularly calm days by a small amount, potentially due to the convergence of the optimization algorithm being sensitive to the input $R_{r s}$.

Algorithm-based glint correction approaches like SOSC and MASC can improve WQP estimation accuracy from spectral radiometry, but this study indicates that a polarizerbased glint correction approach could be a viable alternative, at least for the radiometric 
retrieval of chlorophyll concentration (Figs. 2-10 and 2-11. Table 2.3). The relatively minor improvement seen in $b_{\mathrm{bp} 650}$ when using a polarizer could represent an artifact of our analysis given that the comparison in situ measurements were unpolarized and thus any potential variation over time in the polarization state of the backscattered light would not be captured in the in situ measurements. The observed lower accuracy in $b_{\text {bp650 }}$ could also be due to the lower SNR of the polarizer-corrected data compared to the uncorrected data in the red and NIR (Fig. 2-6), which would result from the lower spectral efficiency of the polarizer in this region of the spectrum. Despite these nuances, a vertical polarizer may still be a viable alternative to algorithm-based glint correction approaches for removing skylight variations, to enable increased accuracy in chlorophyll estimates without requiring the additional wavelengths necessary for glint correction. This equivalent accuracy of a polarizer is particularly noteworthy for researchers who only want to investigate chlorophyll using a single algorithm, because similar accuracy may be achieved by using a less expensive multispectral camera with a polarizer, instead of a hyperspectral imaging spectrometer and software glint correction.

\subsubsection{Implications of view angle on glint correction}

The observed view angle dependence of the quality assurance (QA) of the $R_{r s}$ 's and the accuracy of the WQP estimates derived from those $\mathrm{R}_{\mathrm{rs}}$ 's agreed with high negative correlation and generally followed expectations from the literature for each of the software glint correction approaches. Absent glint correction, a poorer $\mathrm{R}_{\mathrm{rs}} \mathrm{QA}$ and less accurate estimates of chlorophyll were seen at higher view angles (Fig. 2-10), which can be explained by the increasing contribution of the reflected sky radiance with view angle [Mobley, 1999]. Similarly, the two algorithm-based glint removal approaches also exhibited a decrease in the $\mathrm{R}_{\mathrm{rs}}$ QA and an increase in the WQP MAPEs with view angle, likely for the same reason. These two algorithm-based approaches had the highest accuracy estimates and $R_{r s}$ QAs near to $40^{\circ}$, where the contribution of the reflected sky radiance is minimized. Optimal estimation of $\mathrm{b}_{\mathrm{bp} 650}$ from uncorrected data was seen at $\sim 45^{\circ}$ (Table 2.3), but oddly the MAPE slightly decreased with view angle in this case (Fig. 2-10). Overall, when using no glint correction or software glint correction, the $40^{\circ}$ view angle seems to still be optimal for $R_{\mathrm{rs}}$ measurement and WQP estimation.

As expected due to the polarization state of the surface reflected light, when using 
a polarizer to correct for glint there was a general decrease in the MAPE of the WQP estimates, and a general increase in the QA of the $\mathrm{R}_{\mathrm{rs}}$ 's, with view angle. However, the accuracy benefit plateaued near $44^{\circ}$ when estimating both WQPs, which was unexpected due to the increase in the horizontal polarization of the reflected sky-light from a planar surface when approaching Brewster's angle Fougnie et al., 1999, Zhou et al., 2013. At angles ranging from $44-53^{\circ}$ the surface-reflected light of a flat surface is only partially horizontally polarized, so these angles should not be able to achieve the same WQP estimation accuracy, or QA, as Brewster's angle. It is possible that capillary waves or other environmental factors limited the potential accuracy gains of using a polarizer within the $44-53^{\circ}$ view angle range. In summary, when using a polarizer to correct for glint, above a $44^{\circ}$ view angle and approaching Brewster's angle remains optimal.

An added complication related to view angle may be expected in ship- or drone-based imager deployments, which may suffer from significant pitch or roll. Interestingly all three glint correction approaches provided lower MAPE chlorophyll estimates, and higher $\mathrm{R}_{\mathrm{rs}}$ QA, over the entire $40-53^{\circ}$ view angle range than seen in the uncorrected data, despite the sky radiometer only measuring the downwelling skylight centered around a single angle at $45^{\circ}$ from zenith and the lower view angles only providing partially-polarized surfacereflected light for the polarizer-based glint correction approach. The ability of the glint correction techniques to correct for sky glint in these off-angle situations suggests that these approaches could potentially tolerate significant pitch and roll in aerial drone applications and still outperform results made with uncorrected data. One caveat is that our research trained the empirical algorithms [Eqns. 2.4 and 2.5] at each view angle, and so any algorithm calculated at a single view angle may be more sensitive to shifts in observational view angle.

\subsubsection{Practical factors for glint removal from mobile platforms}

Although the precision of the recovered water-leaving radiance signal and the accuracy of the WQP estimates are important factors for determining between the glint correction approaches, practical considerations for implementing each approach must also be noted. The software-based sky glint correction approaches require a sky-looking radiometer, which increases payload weight and thus may reduce deployment range or duration from a mobile platform. These two approaches also require spectral data at additional wavelengths beyond those for computing the WQPs themselves: multiple bands spanning the visible to NIR for 
the SOSC, and a single band in the NIR for the MASC, which may require more complex imagers. The polarizer-based approach requires no additional wavelengths or a sky radiometer, but it does impose additional limitations on the imager including the need to image near to Brewster's angle and perform a new radiometric correction that includes the polarizer in the optical train. Moreover the imager must be sensitive to vertically polarized light, which can be an issue for some grating-based hyperspectral imagers. The above practical limitations require a user to provide forethought about which constraints can be tolerated when choosing a glint correction approach.

Researchers must proceed with care when extrapolating these results for use on lowaltitude mobile platforms such as ships or drones, as the spatial resolution will vary due to spatial smearing from the platforms movement. The minimum and maximum exposure times from our uncorrected data set ranged from $3.8 \mathrm{~ms}$ to $87 \mathrm{~ms}$. In bright conditions $(\sim 3.8 \mathrm{~ms}$ exposure times), the platform could move at $4.5 \mathrm{~m} / \mathrm{s}$ to maintain the $\sim 2 \mathrm{~cm}$ resolution of this study, but on dark days the mobile platform would have to move at $<1 \mathrm{~m} / \mathrm{s}$. Alternatively the exposure time, and therefore captured electrons and SNR, would need to decrease to cover more area. Using a drone with a forward speed of the maximum of $160 \mathrm{~km} / \mathrm{h}$ set by the FAA Federal Aviation Administration (FAA), 2016b, the motion-induced spatial smear under comparable conditions would vary from 0.2 to $4 \mathrm{~m}$, which is much coarser than the resolution used in this paper. The spatial averaging will likely reduce the impact that capillary waves have on changing the surface-reflected light, but the SOSC approach would likely still be optimal at courser resolutions as well, as it has been seen to work well on course resolution radiometer data Lee et al., 2010; Shang et al., 2017.

The results contained within this paper suggest that the available off-the-shelf imaging spectrometers have considerable promise for optical remote sensing of natural water bodies on fine-scales, but it is important to not extrapolate these findings unduly. This study examined only one candidate imaging spectrometer (a Resonon Pika-L), on two specific WQPs ([chl] and $b_{\mathrm{bp} 650}$ ), using two empirically trained multispectral ocean color algorithms, in one region of the coastal ocean, at a single height above the ocean surface on an ideal fixed platform. Deploying such a line scan imaging spectrometer on a moving platform is necessary to generate spatial maps of WQPs, but motion introduces a number of complications not encountered in this study. The auto-exposure algorithm used in our study to maintain the signal level of our imager while imaging homogeneous waters will likely work less well over 
heterogeneous regions such as the coastal ocean, where images may contain land or ships in addition to water. Also, the view angle of the imager was relatively small, which will limit the area that can be mapped by a mobile platform (although the field of view could be increased with a different choice of objective lens). In addition, the long in situ time series allowed for the determination of accurate empirical coefficients which may not be achievable in most field studies. Finally, since only a single radiometric image was used to calibrate the imaging spectrometer, the WQP MAPE is likely higher than would be achievable with perfect radiometric correction. However, the impact of this single radiometric image correction is analogous to vicariously calibrating an imaging spectrometer deployed from a fixed-wing drone platform, where it may only be possible to capture a single image of a calibration target on the ground. In summary, the fixed-tower platform provided a useful test site to collect a range of in situ data to be able to compare between the glint correction techniques at very fine spatial scales, but care should be taken when extrapolating these findings to sensing from mobile platforms as there are many complicating factors.

\subsection{Conclusions}

This study presents a comparison of three glint correction approaches that can be applied to lightweight, low-power imaging spectrometers for fine-scale spatial mapping applications of water quality properties such as $[\mathrm{chl}]$ and $\mathrm{b}_{\mathrm{bp} 650}$. Overall, despite the low spectral SNR of lightweight low-power imaging spectrometers and the fine-scale variations in the ocean surface's reflection coefficient, these currently available imagers appear adequate for providing general fine-scale [chl] estimates over natural water bodies when glint correction approaches are applied. However, when estimating $b_{b p 650}$, the accuracy of the imaging spectrometer seemed to be limited by either the scale of the measurements or the low SNR of the imager when using glint correction approaches. Of the three approaches examined here, a spectral optimization glint correction algorithm both had the highest quality assurance $R_{r s}$ 's and led to the best estimates of both WQPs on centimeter-scale spatial resolutions when applying standard, but empirically trained, ocean color algorithms. We bounded this spectral optimization approach to provide more accurate glint correction results for our dataset. A simple hardware-based polarizer solution provided similar accuracy as the computationally complex spectral optimization approach for recovering [chl] on these fine scales, but was less 
effective with estimates of $b_{b p 650}$. The results of this paper provide a quantitative basis for selection and deployment of the currently available glint correction approaches for fine-scale WQP estimation from an imaging spectrometer.

Tower-, ship-, or drone-based applications of small, lightweight imaging spectrometers in aquatic remote sensing have considerable promise to fill an operational niche between in situ sampling, aircraft-based studies, and satellite ocean color monitoring, by providing fine temporal and spatial resolution data with minimal operational constraints Klemas, 2015]. Low-altitude platforms have an advantage over aircrafts and satellites because they can operate underneath cloud cover, an important consideration in typically cloudy and spatially dynamic ocean regions such as coastal waters Cao et al., 2018, Feng et al., 2017. and the marginal ice zone Engelsen et al. 2002. This study demonstrated that a currently available imaging spectrometer was able to recover WQPs from a fixed-platform on very fine spatial scales, on the order of centimeters, despite the low signal-to-noise ratio measurements from the imaging spectrometer and the complications arising from applying glint correction approaches to waters with fine-scale changes in their surface reflectance characteristics. 


\title{
Chapter 3
}

\section{A simulation framework for evaluating lightweight spectral cameras in drone-based aquatic sensing applications}

This chapter was published in the Optical Society of America's Journal of Applied Optics O'Shea and Laney, 2020] and a revised version is reproduced here.

\begin{abstract}
Optical remote sensing of aquatic environments using aerial drones is becoming more feasible as lightweight, low-power, multi- and hyperspectral cameras increase in availability. Use of these cameras in such applications involves complex trade-offs in optical design and in deployment strategies, and simulations provide a means to examine this multidimensional design space to identify specific limitations on performance for a given measurement scenario. In this paper, such a simulation framework is developed and its use in two realistic aquatic remote sensing scenarios is explored. Such a framework can provide insight not only into uses of existing camera systems, but also aspects of optical design or hardware that would lead to improved accuracy when using such cameras aerially over natural water bodies.
\end{abstract}

\subsection{Introduction}

Modern multi- and hyperspectral cameras now provide environmental researchers with imaging technologies that may have considerable potential for remote sensing applications using small aerial drones [Sigernes et al., 2018]. A number of terrestrial remote sensing applications have been examined to date, and cameras that are lightweight, low-power, and have low signal-to-noise ratios (SNR) may also be suited for fine-scale, drone-based aerial assessment of natural water bodies. At present only a few commercially available multispectral 
cameras have been examined for their potential to remotely recover information on optically active constituents (OACs) from natural water bodies $\mathrm{Su}, 2017$; $\mathrm{Su}$ and $\mathrm{Chou}, 2015$; Totsuka et al., 2019]. Examples of common OACs include suspended or dissolved organic matter, sediment, algae, or pollutants Brando et al. 2012]. In principle, any material that affects the remote sensing reflectance of a water body can be examined on very fine spatial scales using aerial drones. In practice, however, the optical design and characteristics of these cameras set fundamental limitations on the accuracy and precision of such remotely sensed optical measurements. These limitations in turn set further operational constraints on how such cameras can be used effectively on small aerial drones in real-world scenarios to map and monitor natural water bodies.

Accurately measuring the remote sensing reflectance of natural water bodies is challenging because of the interplay between environmental sources of optical noise, such as surface-reflected light (glint) and path radiance, and the optical and electronic design of the particular camera being used Moses et al. 2012a. In such applications, critical camera properties include radiometric resolution, spectral efficiency, dark noise, full-well capacity, and read noise. All of these will affect the accuracy and precision of measured remote sensing reflectances and thus any derived environmental parameter that can be computed using these measured reflectances (Table 3.1) Moses et al. 2012a. Exploratory, real-world assessments have been used to identify the effect of individual parameters for satellite-based sensors (such as digitization noise, e.g. Hu et al. [Hu et al., 2001|), but comparable trial-and-error assessment of an individual camera's optical and electronic characteristics influence on the accuracy and precision of all OAC estimates is effectively intractable. With satellite-based aquatic optical remote sensing the resources are available to perform extensive assessments and analyses to gauge the impact of the optical design on the accuracy and precision of OACs of interest (e.g. HICO [Moses et al., 2012a, 2015], SeaWiFS [Hu et al., 2001]). Such extensive validation efforts are impractical for assessing large numbers of drone-deployable hyperspectral camera and sensing scenario combinations, because each individual combination will likely only be used by, at most, a few researchers. However, user run simulations can provide much of the information needed to evaluate whether a given camera is suitable for a specific drone-based application. 


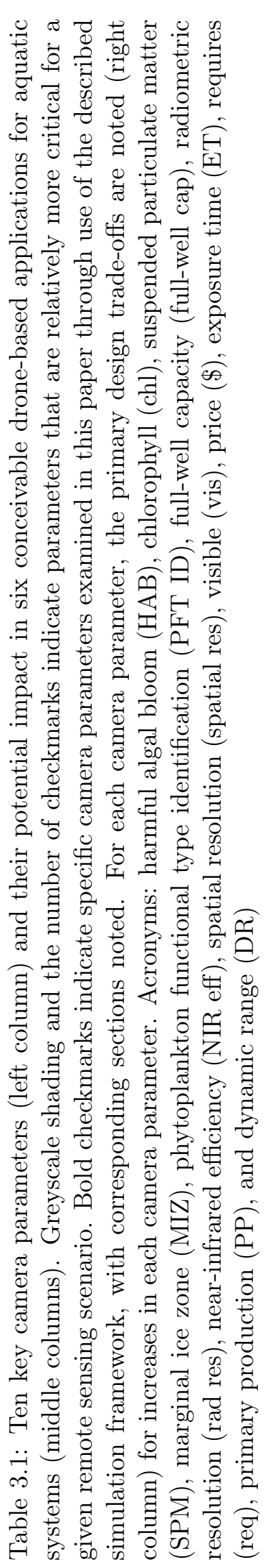

\begin{tabular}{|c|c|c|c|c|c|c|c|c|c|c|c|}
\hline & 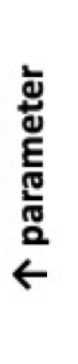 & 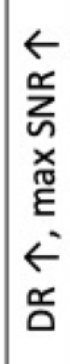 & 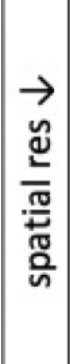 & 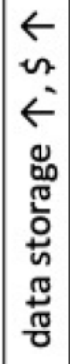 & 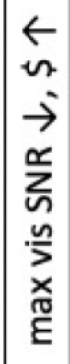 & 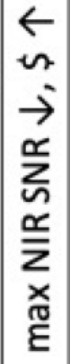 & $\begin{array}{l}\vec{c} \\
\underset{\sim}{u}\end{array}$ & 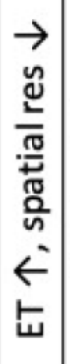 & $\begin{array}{l}\rightarrow \\
\sim \\
\leftarrow \\
\leftarrow \\
\leftarrow\end{array}$ & 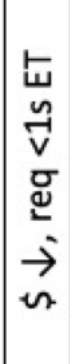 & $\vec{n}$ \\
\hline & 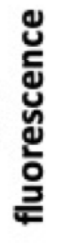 & $\overrightarrow{3}$ & $\overrightarrow{>}$ & 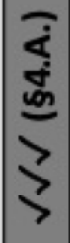 & 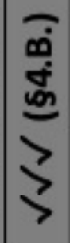 & $>$ & $\overrightarrow{>}$ & $\overrightarrow{3}$ & $\overrightarrow{3}$ & $>$ & $\overrightarrow{3}$ \\
\hline & $\frac{2}{2}$ & 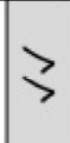 & $\overrightarrow{\geqslant}$ & $>$ & $\overrightarrow{3}$ & $>$ & $>$ & $\overrightarrow{\geqslant}$ & 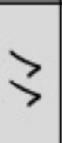 & $>$ & $>$ \\
\hline $\begin{array}{l}\frac{N}{\Sigma} \\
\vdots \leq \\
\vdots \\
0\end{array}$ & 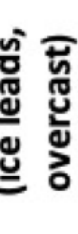 & 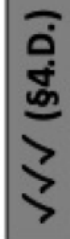 & 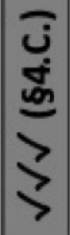 & $\geqslant$ & $\geqslant$ & $\overrightarrow{3}$ & $\overrightarrow{3}$ & $>$ & $\overrightarrow{3}$ & $\overrightarrow{3}$ & $>$ \\
\hline 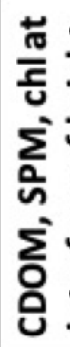 & 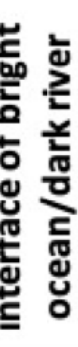 & 3 & $\overrightarrow{>}$ & $>$ & $\overrightarrow{3}$ & 3 & $\overrightarrow{>}$ & $\overrightarrow{3}$ & $\vec{z}$ & $>$ & $>$ \\
\hline 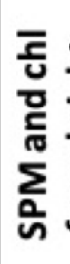 & 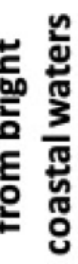 & $>$ & $\geqslant$ & $>$ & $\overrightarrow{3}$ & $\overrightarrow{>}$ & $\overrightarrow{3}$ & $\overrightarrow{>}$ & $\overrightarrow{\rangle}$ & $>$ & $>$ \\
\hline & 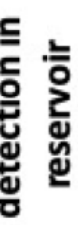 & & $>$ & $>$ & $\geqslant$ & 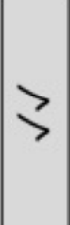 & $\overrightarrow{3}$ & $\geqslant$ & 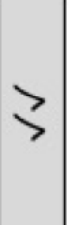 & $>$ & $>$ \\
\hline 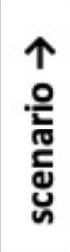 & 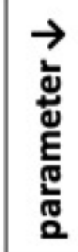 & $\begin{array}{l}0 \\
\frac{2}{0} \\
\frac{0}{0} \\
\overline{0} \\
\frac{1}{3} \\
\frac{1}{2}\end{array}$ & 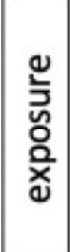 & 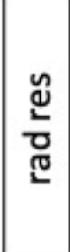 & $\begin{array}{l}\frac{w}{\alpha} \\
\frac{\tilde{c}}{z} \\
\frac{\alpha}{z}\end{array}$ & 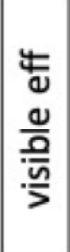 & 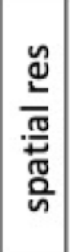 & 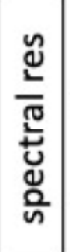 & 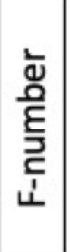 & 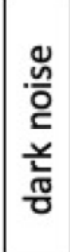 & 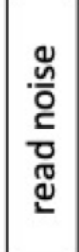 \\
\hline
\end{tabular}


Described in this paper is a computational framework that allows designers to simulate how differences in single or multiple camera parameters affect final parameter estimates for a particular remote sensing scenario in aquatic systems. Of the range of key camera parameters listed in Table 3.1, this paper explored how the simulation framework could be used to assess potential changes in four of these camera parameters, and their impact in two realistic aquatic remote sensing scenarios (bold text, indicating subsequent sections). Finally, how those results could feed back into camera selection, adaptation, and deployment for each scenario is discussed.

\subsection{Overview of Key Camera Parameters and Environmental Considerations}

Before outlining a simulation framework for using hyperspectral cameras for aquatic applications, it is first necessary to overview the fundamental sources of noise in camera systems as well as other operational factors related to their use in drone-based scenarios, which together dictate the overall accuracy and precision for radiometrically estimating OACs of interest in natural water bodies.

Multi- and hyperspectral cameras suffer from a range of noise sources inherent to their electronics and optics, each of which reduces the on-board spectral signal-to-noise ratio (SNR) (Table 3.1). Assuming a high intensity signal measured with a short exposure time, these noise sources could be ranked from least to most substantial: dark noise, read noise, quantization noise (as set by the radiometric resolution), and photon-shot noise (which is limited by the full-well capacity). The contribution of dark noise increases both with exposure time and with temperature Gow et al., 2007; Farrell et al., 2012], and dominates at long exposure times. Read noise occurs during the electronic readout of the electrons contained in each pixels full-well [Chen et al. 2009]. Both dark noise and read noise exhibit the largest impact on the SNR when the measured signals are of low intensity. Quantization noise is uniformly distributed and is the result of round-off error from the digitization of the number of electrons Boncelet, 2005. Since quantization noise is low in magnitude and not time varying, it also has a more prominent effect on low intensity signals. Shot-noise is the

only source of noise that is dependant on signal magnitude and typically dominates the noise at high intensity levels. Due to the discrete nature of electrons and photons, shot-noise has 
a Poisson distribution. The relative effects of optical and electronic sources of noise change as a function of the intensity of the measured signal, which within a hyperspectral image of a natural water body can vary significantly.

The optical and electronics noise sources directly affect the estimated water-leaving radiance, the noise can then propagate through software algorithms to produce imprecise estimates of OACs and inaccurate correction of surface reflected light (glint) Levin et al., 2005; Levin and Levina, 2007]. OAC algorithms typically utilize band ratios of the remote sensing reflectance, which is the camera measured radiance divided by the irradiometer measured irradiance. It is through this pathway that the noise on the water-leaving radiance signal measured on the camera could lead to imprecise and inaccurate measurements of OACs. Software glint correction approaches also rely on measurements of the remote sensing reflectance, typically in the low signal-to-noise ratio NIR bands, so they too could theoretically suffer from imprecision and inaccuracy of the camera measured water-leaving radiance signal. Overall, the inherent noise sources in the camera system can be accentuated, depending on the camera measured signal, and propagated through to estimates of the OACs.

The ability to deploy drones in a variety of environmental conditions and above waters containing unique optically active constituent combinations results in a wide range of shapes and intensities of the measured spectral water-leaving radiance signal. From an environmental standpoint sunny and cloudy conditions heavily impact the downwelling radiance, and therefore the upwelling water-leaving radiance intensity and spectra. Higher concentrations of suspended particulate matter (SPM) increase the reflectance across the spectrum Kirk, 1994, notably in the NIR. Increases in the chlorophyll (chl) concentration typically reduce the spectra in the blue and increase the reflectance in the green and near-infrared (NIR) Clarke et al., 1970, Morel and Prieur, 1977. An increased contribution of colored dissolved organic matter (CDOM) increases the absorbance in the blue and green Morel and Prieur, 1977; Gholizadeh et al. 2016], which decreases the water-leaving radiance in this part of the spectrum. The combined environmental conditions and $\mathrm{OAC}$ concentration ranges result in unique water-leaving radiance spectra and, in extension, unique sensor requirements for each ocean color scenario.

The wide range of water-leaving radiance signals and remote estimation tasks results in unique impacts of the selectable camera parameters on the remote sensing products (e.g., 
Table 3.1. For example, harmful algal blooms (HABs) in reservoirs can produce a wide dynamic range of reflectances in the spatial domain, due to a wide range of algal concentrations on fine-scales. Therefore, they will require high full-well capacities to accurately estimate $\mathrm{OAC}$ concentrations from the lowest reflectances. If a user instead wanted to sense chlorophyll in the marginal ice zone, by measuring from the oceanic signal emanating from between sea ice leads, the spatial resolution (and therefore exposure time and F-number) would become much more important than the dynamic range. The finer spatial resolution would allow for the selection of many more ice-free pixels, which is useful as ice has a much higher reflectance than the dark ocean waters. For another remote estimation task, phytoplankton functional type identification (PFT ID), spectral resolution becomes the key parameter, as increasing the spectral resolution, up to a point, provides additional information that enables separation of the absorption spectra of the different functional types Aiken et al., 2014. Finally, for measuring the low intensity chlorophyll fluorescence signal, the radiometric resolution may become the limiting factor, as it is required to differentiate between small changes in the chlorophyll fluorescence signal. Although it is possible to discuss qualitatively how the individual parameters may effect a range of sensing scenarios without using simulation, a simulation framework is required to assess how any individual parameter would quantitatively impact the remote estimation accuracy in a specific region, and thereby inform the user on how to select and deploy a commercially available spectral camera system.

\subsection{Representing Drone-based Hyperspectral Remote Sensing Scenarios in A Simulation Framework}

The overall process for how light radiating from a water body is ultimately used to compute any given $\mathrm{OAC}$ can be represented graphically (Fig. 3-1, top), from which a simulation framework can be developed (Fig. 3-1, bottom). With the former, the influences of individual camera parameters can be clearly identified, as can the points at which individual sources of noise are added to the signal. These can then be mapped directly to steps within the simulation framework. The framework used here is adapted from Moses et al. Moses et al., 2012a (their Fig. 1), by considering a drone just below $100 \mathrm{~m}$ altitude where path radiance does not need to be corrected [Mueller and Austin, 1995], as its contribution to the 
total radiance is significantly reduced. Not every real-world consideration has been included in the simulation framework described here, and deviations are identified below. Although the simulation framework could in principle be implemented in any language, MATLAB was the programming environment chosen to implement the below steps.

\subsubsection{Ocean color formation}

The first step of the optical pipeline in the top of Figure 3-1 is the propagation of the irradiance $\left(E_{d}\right)$, which downwells and interacts with the OACs and reflects off of them to form the water-leaving radiance $\left(\mathrm{L}_{\mathrm{w}}\right)$. Surface-reflected light (glint) is added to the water-leaving radiance, as it leaves the ocean and enters the atmosphere, to form the total water-leaving radiance $\left(\mathrm{L}_{\mathrm{t}}\right)$.

The simulation framework accepts simulated or measured ocean color data as inputs. Both are useful in their own way. Hydrolight simulated datasets allow for estimation of the light levels in regions where no ocean color data is at hand. Real-world ocean color data collected at high SNR provide more accurate quantitative assessment of the relative impacts of the camera parameters, but require field tests for accurate collection.

In this paper, two different ocean color scenarios are assessed using Hydrolight simulated ocean color data, as they provide simultaneous acquisition of all ocean color data, and in-situ 'truth' values without errors due to environmental fluctuations. Table 3.2 shows the ranges of optically active constituents simulated for each dataset in Hydrolight. The first dataset uses concentration ranges which correspond to realistic values from Case II waters, where chlorophyll, suspended particulate matter, and colored dissolved organic matter can vary significantly [Morel and Prieur, 1977]. In the second dataset, high algal biomass waters with chlorophyll concentration ranging from $0.05-20 \mathrm{ug} / \mathrm{l}$ imitate that of a chlorophyll bloom seen in the West Antarctic Peninsula [Massom et al., 2006]. This second dataset is simulated for overcast conditions with a solar elevation angle of $30^{\circ}$, representative of conditions in austral summer near the West Antarctic Peninsula. These two simulated ocean color datasets can then be propagated through the optical pipeline. 
Table 3.2: Hydrolight Simulated OAC Concentration ranges. The 'Case II' entry simulates a Case II region, where light absorption cannot be ascribed primarily to chlorophyll-containing organisms. 'Antarctic bloom' indicates a dataset simulating chlorophyll concentrations spanning the range observed during a 2001-2002 phytoplankton bloom in the West Antarctic Peninsula Massom et al. 2006]. In the 'Antarctic bloom' dataset, CDOM varies proportionally with the chlorophyll concentration $(\propto \mathrm{chl})$.

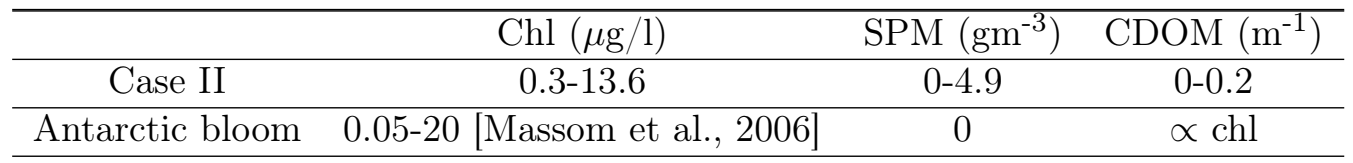

\subsubsection{Optical propagation through the atmosphere into a camera system at altitude}

In the real-world scenario, once exiting the ocean-atmosphere interface, the water-leaving radiance propagates through a path length of atmosphere where it is imaged by the dronemounted camera. Path radiance, which is a function of the drone's altitude, is added to the total water-leaving radiance signal and transmission loss due to traveling through the atmosphere occurs before being measured by the camera.

The power measured on the camera's detector is a function of the area of the ocean's surface being imaged. To determine the amount of photons incident on the camera's detector, first the total water-leaving radiance is converted to power on the detector [Eq. 3.1] Mobley et al. 2016a.

$$
P_{\text {detector }}=L_{T} * \pi * \frac{\left(r_{\text {aperture }}\right)^{2}}{(\text { slant range })^{2}} * A_{\text {surface }} * \cos \left(\theta_{\mathrm{v}}\right) * O E * \Delta \lambda
$$

Here $P_{\text {detector }}$ is the power captured on the whole detector, $L_{\mathrm{T}}$ is the total spectral waterleaving radiance, $\pi$ times the radius of the aperture $\left(r_{\text {aperture }}\right)$ divided by the slant range is the solid angle of the sensor. The slant range is the distance from the camera to the imaged water. $A_{\text {surface }}$ is the surface area of the water, $\theta_{\mathrm{v}}$ is the view-angle, $\cos \left(\theta_{\mathrm{v}}\right)$ corrects for the off-angle measurement of the area, $\mathrm{OE}$ is the optical efficiency of the camera systems optical components for the specific wavelength, and $\Delta \lambda$ is the spectral bandwidth captured by this detector. The power per pixel $\left(P_{\text {pixel }}\right)$ is derived by dividing $P_{\text {detector }}$ by the total number of pixels.

One difference between the conceptualized optical model (Fig. 3-1, top) and its representation in the computational simulation (bottom) is that path radiance is not added to the 


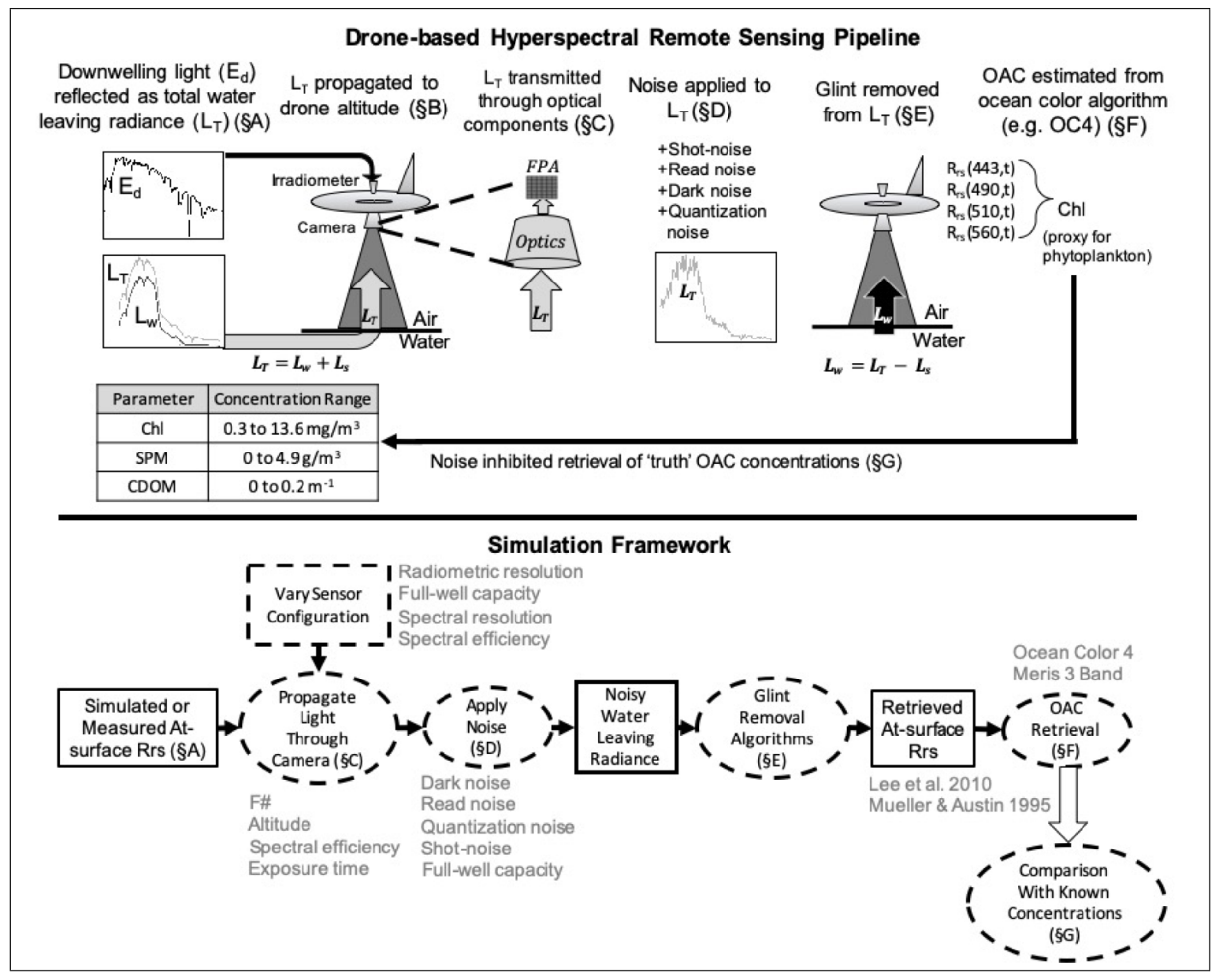

Figure 3-1: Top: the optical propagation of water-leaving radiance through a drone-based hyperspectral imaging system. The water-leaving radiance (A) first propagates through a short path within the atmosphere $(\sim 100 \mathrm{~m})$ and then passes into the imaging optics of an airborne sensor at a given altitude (B). This radiance is then transmitted through the imaging optics of the camera system (C) and imaged by the focal plane array (FPA), which adds optical and electronic noise to the signal (D). If desired, an algorithm can be applied to correct for glint artifacts before computing the measured, noisy, total waterleaving radiances at different wavelengths $(\mathrm{E})$. These are then used in ocean color algorithms to compute any given $\mathrm{OAC}$ of interest $(\mathrm{F})$. The overall effect of the camera system noise can be determined by comparing these remote sensing estimates to the 'truth' values used to initiate this simulation $(G)$, which are derived either from in-situ measurements or input data from aquatic optical modeling tools such as Hydrolight (Sequoia Scientific Mobley and Sundman, 2008|). Bottom: the mapping of this physical description onto the simulation framework. Grey text indicates selectable parameters of camera systems that affect each step.

total water-leaving radiance signal. Another difference is that transmission losses are not subtracted from the total water-leaving radiance. This can be justified because the allowable altitudes of small drones is typically low, such as the maximum 400 feet (roughly $122 \mathrm{~m}$ ) 
set by the Federal Aviation Administration (FAA) in the United States Federal Aviation Administration (FAA), 2016a). Over such distances the atmospheric transmittance and path radiance contributions will be relatively minimal, though Mueller and Austin Mueller and Austin, 1995] do mandate correcting for both when slant ranges exceed 100 meters (which in the example use cases covered in this paper are slightly exceeded due to the off-nadir view-angle). If the transmission and path radiance effects were not ignored, the main effect they would have would be on slightly changing the exposure time required to saturate the full-well capacity. If required for any future simulation, this minimal path radiance can be corrected for using one of a variety of atmospheric compensation models [Griffin et al., 2003]. For the use cases examined here, path radiance will be ignored as a source of error although it could, in principle, be added to $L_{\mathrm{t}}$ and propagated through the simulation framework if desired.

\subsubsection{Transmission through an optical train}

The power incident on the detector is then propagated through the camera's spectral efficiency and imaged by an individual pixel on the camera's focal plane array (FPA). The number of electrons imaged in each pixel is a function of the exposure time and quantum efficiency [Eq. 3.2].

$$
N_{\text {electrons }}=P_{\text {pixel }} * t * \frac{\lambda}{h * c} * Q E
$$

The power of a given pixel $\left(P_{\text {pixel }}\right)$ is converted to energy by multiplying by time $(t)$, then converted to number of photons by multiplying by wavelength $(\lambda)$ and dividing by Plank's constant $(h)$ and the speed of light $(c)$ Mobley et al., 2016a. Finally, the number of electrons is computed through multiplication with the spectral quantum efficiency (QE) for a given camera's imaging sensor.

In the example use cases covered in this paper, light is simulated passing through an idealized camera system with characteristics roughly comparable in performance to a commercially available hyperspectral line-scan unit (Resonon Pika L). The simulated camera system has $2 \mathrm{~nm}$ spectral resolution, hardware spatial and spectral binning capabilities, an f-number of 2.4, an instantaneous field of view (IFOV) of 0.71 mrads, a full-well capacity of 35,000 , and a focal length of $17 \mathrm{~mm}$. The spectral efficiencies of the optical components (which comprise the $\mathrm{OE}$ ) are estimated from analogous components available from commer- 
cial optics suppliers (e.g. Thorlabs Inc., New Jersey USA). The QE is set following the spectral efficiency of the camera's focal plane array (FPA).

\subsubsection{Application of noise}

After the input radiance is propagated through the simulated camera system and onto the focal plane array, simulated noise is applied to the signal. As stated, these simulated noise sources include dark noise, read noise, shot-noise, and quantization noise. In the simulations used in this paper, the read noise (Noise ${ }_{\text {read }}$ ) [Eq. 3.3 was modeled as a Gaussian-distributed random value with a mean of 0 and RMSE as provided by a manufacturer Vliet et al., 1998.

$$
\text { Noise }_{\text {read }}=\operatorname{Gaussian}(0, R M S E)
$$

Within the simulation the read noise can readily be modified to include an offset value or employ a different statistical distribution if desired, such as Poisson [Boncelet, 2005]. Dark current shot noise dependent on exposure time is also added, following a Poisson distribution [Eq. 3.4].

$$
\text { Noise }_{\text {dark }}=\text { Poisson }\left((E C * E T)^{.5}\right)
$$

Here, Noise $_{\text {dark }}$ is the dark noise, EC is the electron current, and ET is the exposure time. Values for EC can be obtained for actual cameras, from manufacturer's data or calculated through in-lab tests. As with the read noise, users can simulate dark noise with different statistical distributions if desired, such as a Gaussian [Boncelet, 2005]. Next, Poissondistributed shot-noise is added [Eq. 3.5.

$$
\text { Noise }_{\text {shot }}=\operatorname{Poisson}\left(N_{\text {electrons }}\right)
$$

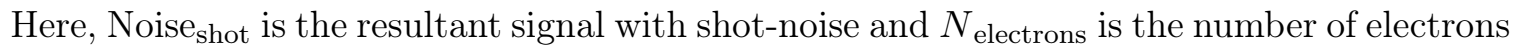
measured on the sensor before shot-noise was added. It is important to take into account that the noise is Poisson distributed, instead of the typically assumed Gaussian distributed. In aquatic remote sensing the measured water-leaving radiances can be quite low in certain parts of the spectrum (e.g., below 1000 electrons on the camera), where differences between these two distributions can become significant.

Finally, quantization noise $\left(\right.$ Noise $\left._{\text {quantization }}\right)$, which arises due to quantization of the 
electrons into bits, is added. The theoretical quantization noise can be modeled as being uniformly distributed Boncelet, 2005. Therefore, the variance has a value which depends upon the full-well capacity (FC) and radiometric resolution (RR) [Eq. 3.6.

$$
\text { Noise }_{\text {quantization }}=\left(F C / 2^{\mathrm{RR}}\right)^{2} / 12
$$

In this simulation framework, quantization noise is not strictly implemented according to Eq. 3.6 Gow et al. 2007. After applying these first three noise sources, the electrons are converted to bits by first dividing the number of electrons by the camera's full-well capacity (FC), next multiplying by the maximum representable value by the analog-to-

digital converter $\left(2^{\mathrm{RR}}\right)$, and finally rounding the result to the nearest integer [Eq. 3.7.

$$
\text { Signal }_{\text {quantized }}=\operatorname{round}\left(2^{\mathrm{RR}} *\left(\text { Noise }_{\text {shot }}+\text { Noise }_{\text {dark }}+\text { Noise }_{\text {read }}\right) / F C\right)
$$

This implementation is more realistic than the theoretical approximation presented in Eq. 3.6, as the impact of quantization varies for each simulated measurement.

In the simulation, since the quantum efficiency of the camera system and transmission efficiency of the optical elements is known, the resulting 'noisy' measured signal (Signal ${ }_{\text {quantized }}$ in Eq. 3.7), expressed in bits, can be used to solve for the $\mathrm{L}_{\mathrm{T}}$ as measured by the noisy and quantized camera system. In other words, a radiometric calibration can be applied to estimate the radiance from the noisy signal.

\subsubsection{Implementation of glint correction algorithms}

After solving for the noisy, measured total water-leaving radiance $\left(\mathrm{L}_{\mathrm{T}}\right)$, the surface-reflected light (glint) must be removed to recover the water-leaving radiance $\left(\mathrm{L}_{\mathrm{w}}\right)$. A variety of glint removal algorithms exist and each has it's own assumptions and requirements for use. One example algorithm is a NIR subtraction algorithm originally developed by Mueller and Austin Mueller and Austin, 1995; Mobley, 1999. This method assumes a reflectance coefficient based on the view angle of the instrument and it also assumes that the radiance at $750 \mathrm{~nm}$ is solely due to glint. To implement the algorithm, measurements of the downwelling sky radiance and the water leaving radiance at $750 \mathrm{~nm}$ are required.

For the simulation, the Mueller and Austin glint removal approach Mueller and Austin, 1995] was implemented as in Mobley Mobley, 1999. 


\subsubsection{Estimation of OAC concentrations}

Numerous algorithms have been developed to interpret spectral differences in a remotely sensed reflectance signal to estimate OACs of interest. In aquatic studies a commonly measured $\mathrm{OAC}$ is the concentration of suspended chlorophyll, which is an estimator for the biomass of phytoplankton in a water body. A representative algorithm for chlorophyll is 'Ocean Color 4' (OC4), developed for use with satellite-sensed ocean spectral remote sensing reflectance data. This algorithm exploits the increase in the remote sensing reflectance seen in the green $(560 \mathrm{~nm})$ relative to the blue $(443,490,510 \mathrm{~nm})$ with increasing concentrations of chlorophyll, by utilizing a maximum band ratio (MBR) approach [Eq. 3.8.

$$
M B R=\left(\frac{\max \left(R_{\mathrm{rs}}(443), R_{\mathrm{rs}}(490), R_{\mathrm{rs}}(510)\right)}{R_{\mathrm{rs}}(560)}\right)
$$

Here, $R_{\mathrm{rs}}$ is the remote sensing reflectance at specific wavelengths (expressed in $\mathrm{nm}$ ). A log-space relationship between the MBR and chlorophyll concentration can be obtained by utilizing fit coefficients $\left(C_{\mathrm{n}}\right)$ [Eq. 3.9.

$$
\begin{aligned}
& \log _{10}([c h l])=C_{4} \log _{10}^{4}(M B R)+C_{3} \log _{10}^{3}(M B R)+ \\
& C_{2} \log _{10}^{2}(M B R)+C_{1} \log _{10}(M B R)+C_{0}
\end{aligned}
$$

These coefficients are typically determined empirically.

Another OAC of interest is the chlorophyll fluorescence line height (FLH), which involves not the absorption properties of this pigment but its spectrofluorescence. In two example use cases for how such hyperspectral cameras can be used to measure FLH (Section 3.4), the fluorescence line height algorithm of Abbott and Letelier Abbott and Letelier] was used [Eq. 3.10].

$$
F L H=L_{676}-\left(L_{746}+\left(L_{656}-L_{746}\right) * \frac{(746-676)}{(746-665)}\right)
$$

Here $L$ is the normalized water-leaving radiance at specific wavelengths in $\mathrm{nm}$ Gordon and Voss, 2004]. The output of this algorithm (the FLH) is then typically used to estimate chlorophyll fluorescence efficiency and primary productivity. 


\subsubsection{Comparing simulated to known OAC concentrations: statistics}

Simulated estimates for OACs are compared to any known in-situ ('truth' or actual) values by computing the median absolute percent difference (MAPD) [Eq. 3.11] [Cui et al. 2013].

$$
M A P D=100 \% * \exp [\text { median } \mid \ln (\text { Estimated } / \text { Actual }) \mid]-1
$$

The MAPD error metric equally weights errors relative to the actual value, which is necessary for comparing estimation accuracy over large dynamic ranges. This metric can also be computed between a high SNR sensor and the low SNR sensor to determine how large of an effect the low SNR has on the derived remote sensing product.

\subsection{Example Use Cases}

In this section, several qualitatively impactful parameters (bold check marks in Table 3.1) were quantitatively evaluated, to demonstrate the utility of the framework for selecting commercially available cameras for specific remote sensing tasks. These test cases reveal how the simulation framework can be used for specific remote sensing scenarios to determine the impact of a single parameter on the remote sensing product, select between different camera systems, estimate the optimal operational parameter (e.g., exposure time) before deploying the system, and reveal camera parameter imposed limitations on the remote sensing accuracy.

\subsubsection{Modeling the required radiometric resolution of cameras to re- motely estimate chlorophyll FLH}

Estimation of chlorophyll FLH requires wavebands in the red section of the spectrum [Eq. 3.10, which in real-world aquatic systems typically have a lower magnitude than the blue and green sections of the spectrum. Since the FLH depends on the low magnitude red measurements, it will be more susceptible to quantization noise, which is inversely proportional to the radiometric resolution [Eq. 3.7] and independent of the signal level. Since the radiometric resolution could theoretically limit the accuracy of the FLH estimates, it is worth investigating the quantitative impact of the radiometric resolution using the simulation framework before selecting a camera system for FLH measurement. 
To determine the quantitative impact of the radiometric resolution on the estimated FLH, Hydrolight simulated ocean color data were passed through the optical simulation framework and evaluated a simulated cameras FLH estimation accuracy. The Hydrolight simulated water-leaving radiances have OAC concentrations as defined in the first dataset, 'Case II', in Table 3.2. These OAC concentrations mimic a Case II scenario, with a wide range of chlorophyll, suspended particulate matter, and colored dissolved organic matter values. The simulated water-leaving radiance is propagated through the simulation framework as in the bottom of Figure 3-1, with the camera system that approximates the characteristics of a representative hyperspectral line-scan camera (similar to the Resonon Pika L, section 3.3.2. . Using the same camera system for each run, the radiometric resolution was varied to determine its impact on the FLH measurement accuracy. The exposure time was set to fill the full-well to roughly one half its max value, to stay away from the saturation region and allow for a wider range of environmental fluctuations without saturation. The MAPD is used as a metric to evaluate the accuracy of the simulated camera's measured FLH, it is calculated between the FLH measured from the optically noiseless (i.e., ideal case) and the FLH estimated from the optically noisy (including shot-noise, read noise, quantization noise, etc.) Mueller and Austin corrected radiance data. The standard deviation of the MAPD is also calculated on 30 individual runs, each with its own random noise sources. The MAPD and standard deviation quantitatively represent the error induced by the combined electronics and optics noise sources, and illustrate how the radiometric resolution limits the estimated FLH accuracy.

Results from this simulation (Fig. 3-2, VIS-blazed) inform users about how a single camera parameter, in this case radiometric resolution, affects the accuracy of a given remote sensing product of interest, in this case chlorophyll FLH. In these simulated results, a radiometric resolution below 10 bits degrades accuracy in FLH estimates (Fig. 3-2, VIS-blazed) and above 10 bits MAPD plateaus at $\sim 9 \%$, suggesting that above 10 bits the radiometric resolution is negligible in comparison to the other noise sources (e.g., shot-noise, read noise, dark noise). Below 10 bits the noise increases exponentially. In this particular OAC remote sensing scenario, radiometric resolution does not limit the accuracy at 10 bits and above, as other noise sources dominate the error. Such quantitative information regarding radiometric resolution could be important if camera data storage or data transfer speeds are near-limiting in a given camera field application. 


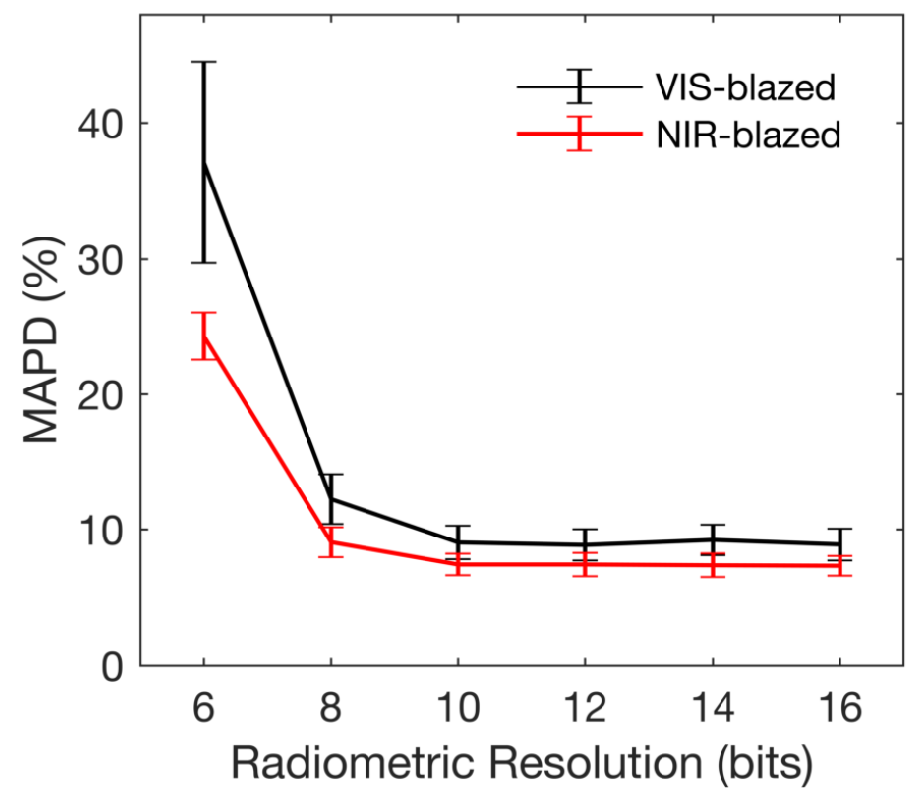

Figure 3-2: The median median absolute percent difference (MAPD) between the optically noisy and noiseless fluorescence line height (FLH) estimate is shown as a function of the radiometric resolution (bit count) and grating blaze type. The VIS-blazed line shows the MAPD when using a visible blazed grating, and the NIR-blazed line shows the MAPD when using a NIR blazed grating instead.

\subsubsection{Determining if a NIR-blazed grating reduces the minimum achiev- able MAPD for measuring chlorophyll FLH}

As noted above, for this particular remote sensing scenario FLH estimates from this simulated camera system are shot-noise limited to $\sim 9 \%$ accuracy as long as the radiometric resolution is 10 bits or higher. Although this error may appear minimal it can compound to further increase inaccuracies in the estimated primary productivity, since the relationship between the FLH and chlorophyll can vary by a factor of 8 Abbott and Letelier. Therefore, it may be ideal to further reduce the error by reducing the shot-noise. By increasing the efficiency in the NIR, the intensity of the captured signal is also increased, thereby reducing the shot-noise limited MAPD. Increasing the efficiency can be achieved by utilizing a NIR-blazed grating optimized for this part of the spectrum. Interestingly, by increasing the intensity of the measured signal, the impact of the quantization noise as a function of radiometric resolution may be reduced in addition to the reduction in the minimum MAPD.

Indeed in this complex camera parameter design space changes in multiple parameters alter the impact that each parameter has on the estimated MAPD. Increasing the efficiency 
in the NIR not only reduces the minimum MAPD to $7.4 \%$ (Fig. 3-2, NIR-blazed), but also reduces the effective impact of the radiometric resolution (Fig. 3-2). When a NIR blazed grating is used instead of a visible grating, an 8 bit ADC achieves a similar error as a 12 bit $\mathrm{ADC}$ on the visible-blazed grating (Fig. 3-2). This simulation outcome demonstrates that changing multiple camera parameters can result in otherwise unforeseeable effects on the overall accuracy for a specific remote sensing product.

\subsubsection{Evaluating the trade-off between spatial resolution and accuracy for the remote estimation of pelagic chlorophyll between spatially heterogeneous Antarctic sea ice floes}

Drone-based ocean color remote sensing tasks in spatially heterogeneous regions (e.g., marginal ice zone, kelp farms, interface of 2 distinct water bodies) sometimes requires both fine spatial resolution data and a large area of coverage. These two requirements are at odds since flight speed is inversely proportional to the spatial resolution (limited by smearing) but directly proportional to the spatial coverage. To maximize area of coverage while achieving the required spatial resolution, users will need to minimize the camera's exposure time. However, if the intensity of the camera's signal is too low, the remote sensing estimation accuracy may be negatively effected. In this scenario, simulation can be useful to estimate the required exposure time to maintain a pre-defined accuracy for the remote estimation task, while simultaneously capturing fine spatial resolution data over a large spatial extent.

One example spatially heterogeneous remote sensing task that simultaneously requires coverage over a large spatial extent and fine spatial resolution is estimating the environmental effects on the primary production in high sea ice cover in the Southern Ocean. A recent remote sensing based study utilizing SeaWiFS ocean color data showed that the phytoplankton blooms in the marginal ice zone have a strong relationship with wind speed Fitch and Moore, 2007]. Their study was limited by the spatial and temporal resolution, at $1 \mathrm{~km}$ and 30 days respectively, of the SeaWiFS satellite sensor. A drone based survey could determine at what sea ice cover percentage the relationship stopped holding and could achieve finer temporal resolution data (since it would fly underneath cloud cover). Additionally, the drone platform could provide finer spectral resolution data, which could enable comparison of the phytoplankton community structure and how that changes with wind speed. Clearly, the higher temporal, spatial, and spectral resolution data provided from a drone-borne hy- 
perspectral camera could substantially increase the efficacy of studies focused on primary producers in the Southern Ocean.

To determine a first order estimate of the spatial resolution required to identify algae in open water within an area of high sea ice concentrations, image processing techniques were applied to a set of images of Antarctic sea ice taken with an RGB camera (raw data provided by Steer et al. Steer et al. 2008). In an example image (Fig. 3-3 areas within the sea ice itself can be seen to contain green color, indicative of the presence of ice algae (panel A). As well, areas of open water between ice floes may also contain some biomass of chlorophyll containing phytoplankton (panel B). To determine the spatial resolution required to distinguish the open water pixels in each image, the image was first thresholded using both the red section of the spectrum and the ratio of blue to red light. Then on the thresholded image, the fraction of water pixels that could be imaged without sea ice contributions at a variety of spatial resolutions were calculated. This process was repeated for 250 images with high sea ice cover in the Weddell Sea, and the fractional non-ice (open water) areas were sorted by sea ice concentration within each image (Fig. 3-4). In areas with high fractional sea ice cover, this analysis suggests that the total areal fraction of open water that can be imaged is significantly reduced as spatial resolution coarsens.

To estimate the effect of exposure time on both the fraction of water that can be imaged and the accuracy of the chlorophyll estimates, a 12 bit instantiation of the hyperspectral camera from the first example use case (4 3.4.1.) was used to image Hydrolight simulated ocean color radiances at a variety of exposure times. The simulated ocean color data was made using concentration ranges from the Weddell Sea dataset (Tab. 3.2). The simulated camera's exposure time varied from $40 \mathrm{~ms}$, where it nearly saturated the full-well capacity, down to $1 \mathrm{~ms}$. The exposure time is proportional to the signal-to-noise ratio and inversely proportional to the spatial resolution (limited by spatial smearing). A 3rd order polynomial relating the Fluorescence Line Height (FLH) to the [chl] is trained on the noiseless FLH and the actual [chl], and then applied to the noisy data to estimate the [chl]. The simulation revealed that the MAPD between the simulated values and the truth values decreased exponentially with exposure time (spatial resolution) (Fig. 3-5. Achieving the highest MAPD requires the longest exposure time, but that requires sacrificing significant portions of the water in images with the highest sea ice cover percentage (Fig. 3-4). If researchers were interested in estimating the pelagic chlorophyll in regions with $99 \%$ concentrations of sea 


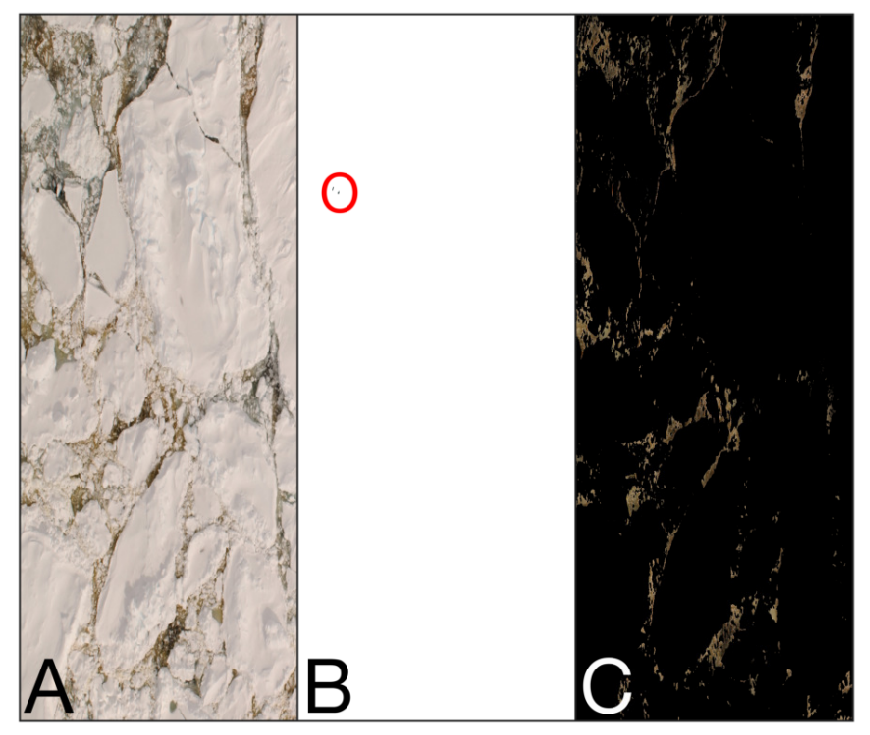

Figure 3-3: An example RGB image of sea ice in the Weddell Sea (panel A), with dimensions of $127 \mathrm{~m}$ by $82 \mathrm{~m}$ (plotted from raw data provided by Steer et al. [Steer et al., 2008]). Pixels representing open water (panel B, black points within red circle) were identified by thresholding the red intensity and blue to red ratio. Additional thresholding can identify areas of algal presence within the sea ice itself (panel C) through the ratio of blue to red light.

ice, they could gain $6 \%$ of fractional area covered by increasing the spatial resolution from 1.6 to $0.8 \mathrm{~m}$ (Fig. 3-4, black dot and dark grey dot). The lower exposure time (spatial resolution) would increase the MAPD in these regions, by $8.9 \%$ (Fig. 3-5, from black dot to dark grey dot). Depending on the application, this trade-off may be worthwhile.

\subsubsection{Estimating the full-well capacity imposed limitations on remotely estimating algal biomass in Antarctic waters}

The previous example focused on estimating chlorophyll in the water column, but up to $50 \%$ of the primary productivity in certain polar regions can occur within the sea ice itself Gosselin et al., 1997; McMinn et al., 2010. A further $50 \%$ of the sea ice primary production typically occurs within $5 \mathrm{~m}$ of the ice edge, and therefore requires high spatial resolution Grose and McMinn, 2003. Unfortunately, the reflectivity of sea ice with algal contributions can be much higher than the water column, due to contributions from the highly reflective sea ice. Therefore, capturing all of the primary productivity by simultaneously imaging both the pelagic and sea ice primary production in this region will require a high dynamic range (full-well capacity). 


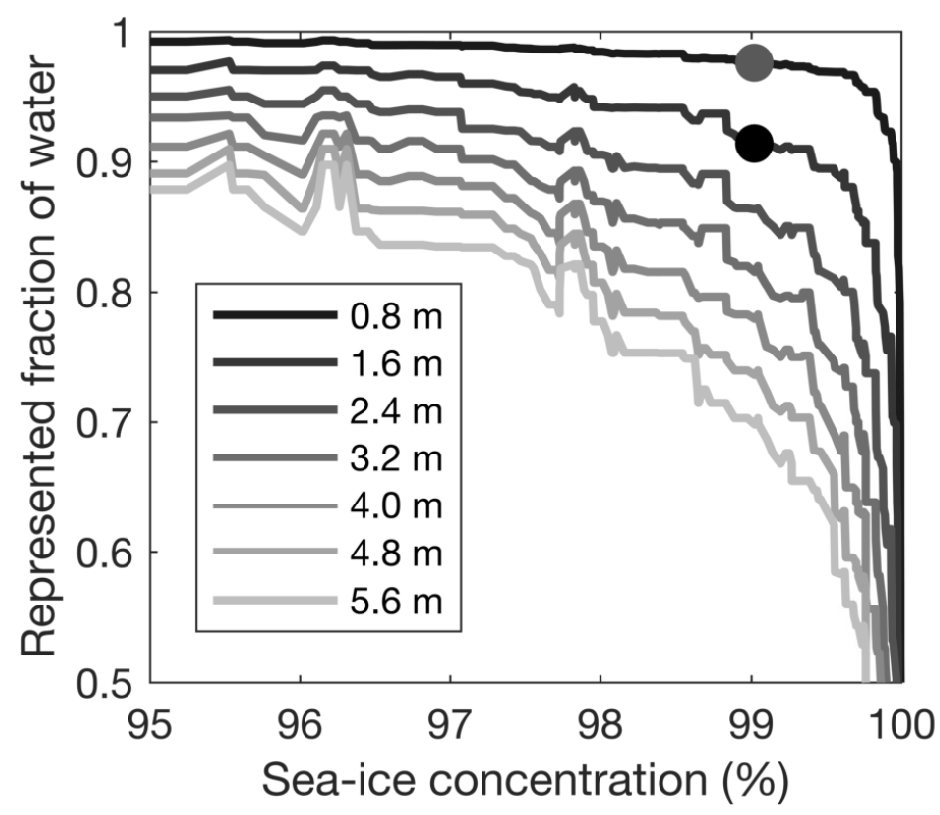

Figure 3-4: The median filtered fraction of the the total water that is imaged without ice contributions as a function of sea ice concentration and spatial resolution for 250 images in high sea ice cover [Steer et al., 2008]. The dark grey and black dots correspond to 0.8 meter and 1.6 meter resolutions, the chlorophyll estimation accuracy at these resolutions is estimated in Fig. 3-5.

The processed red, green, and blue (RGB) image in Figure 3-3 can provide a first order estimate to the dynamic range required to simultaneously image both the pelagic and sea ice algae (Fig. 3-3, B \& C respectively). To compare the intensities of the sea ice and sea ice algae, only the green component of each pixel was compared. This color was chosen because it has the highest intensity in images of sea ice algae, which will limit the saturation of the detector. The 99th percentile green intensity of sea ice algae pixels (Fig. 3-3, C, greenish-yellow pixels) is 83.58. Assuming the sea ice has a reflectance of roughly $80 \%$ [Malinka et al. 2016], and the median green intensity of the sea ice pixels is 134.4, then the 99th percentile brightest green sea ice algae is roughly $49.75 \%$ reflective. This reflectance is calculated by dividing the sea ice reflectance $(80 \%)$ by the median intensity of the sea ice in the green (134.4) and finally multiplying by the 99th percentile green intensity of the sea ice algae (83.58). The simulated waters used to produce Figure 3-4 had a max reflectance of roughly $2 \%$. Since the final point in Fig. 3-4 uses an exposure time that saturates the full-well to $\sim 90 \%$ of its capacity, this wide range could not be captured without saturation of the full-well capacity. Therefore, to not saturate when capturing images of the sea ice 


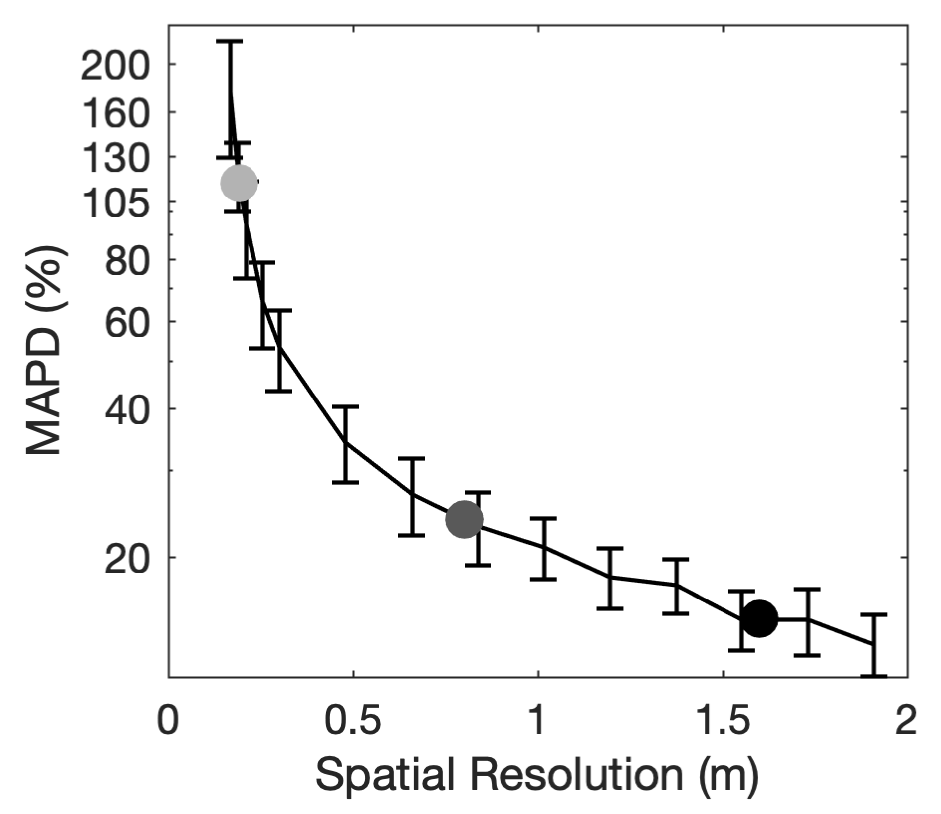

Figure 3-5: The median MAPD and standard deviation (error bars) from the Mueller and Austin Mueller and Austin, 1995 corrected data are plotted versus the along-track spatial resolution (a function of exposure time) of a drone flying at $100 \mathrm{MPH}$, the maximum FAA limited speed [Federal Aviation Administration (FAA), 2016a|. The dark grey and black dots correspond to the spatial resolutions selected in Fig. 3-4 and show a trade-off in fraction of water area represented and MAPD. The light grey dot shows the high error imaging the sea ice algae imposes on the pelagic chlorophyll estimates. The ordinate axis uses log-space to increase visual clarity by separating the error bars at the lowest spatial resolutions.

algae, a decrease in the exposure time (proportional to spatial resolution in Fig. 3-4) of a factor of at least $25(\sim 49.75 \% / 2 \%)$ is required. With no changes in the camera system, this would limit the estimation accuracy of pelagic primary production to $\sim 114 \%$ MAPD (Fig. 3-5, light grey dot). Therefore, the camera system requires re-engineering to be able to simultaneously estimate primary production in both the ocean and the sea ice.

\subsection{Discussion}

Extensive guidelines for SNR requirements have been developed for aquatic remote sensing using satellites [Mueller et al., 2003; Lucke et al., 2011], but these will likely be difficult to apply for drone-based use of hyperspectral cameras for similar applications. Recent work has examined SNR characteristics of drone-compatible hyperspectral cameras using field observations in the context of estimating chlorophyll biomass and particulate backscatter 
O'Shea et al. 2020]. Such field-oriented research suggests that computational simulation frameworks, like the one presented here, can play a valuable role in quantitatively assessing the effect of low SNR in specific wavelengths, on a specific remotely sensed environmental variable of interest. The example use cases presented in this study demonstrate how specific individual camera parameters can be isolated and examined via simulation to determine their influence on these intended remote sensing products. The same approach may be valuable for weighing a candidate spectral camera system with respect to its performance parameters, for choosing between multiple commercially available cameras, for setting camera operational parameters, and for uncovering potential engineering limitations in drone-based spectroradiometric imaging of natural water bodies.

In the first use case, this simulation framework was used to investigate the impact of radiometric resolution, a single performance characteristic, in an application to measure the very low intensity chlorophyll fluorescence emission of natural waters. Since quantization noise decreases exponentially with increasing radiometric resolution [Eq. 3.6], an exponentially decreasing impact on the FLH estimates was expected. The simulation demonstrated how this effect can be specifically quantified in terms of MAPD given user choices in camera radiometric resolution (Fig. 3-2). For this use case, choosing cameras with radiometric resolutions greater than 10 bits will provide no additional improvement in MAPD, and error in this range becomes dominated by other sources of noise, e.g. shot-noise. Without such a simulation, the threshold at which increased radiometric resolution improves MAPD for this specific task would be very time consuming to determine experimentally. This example quantifies exactly how extra precision in digitizing is effectively lost in the electronics and shot-noise. In such a scenario a camera system design that optionally allows greater bit-resolution may be instead operated at a lower resolution, e.g. to store more data during a drone survey without loss of ultimate accuracy in estimated FLH. Similarly, lower radiometric resolution data can be downloaded off the camera more rapidly during flight, thus enabling faster imaging speeds again with no loss of accuracy in FLH. From the perspective of accuracy in the intended remotely sensed OAC (here, FLH), this analysis may provide the designer insight into the expected accuracies in drone-estimated FLH, which with for example 10-bit resolution may be unnecessarily precise given known environmental variability in this signal Abbott and Letelier. In such cases 8-bit resolution may be sufficient, for example. Such considerations would be challenging to examine comprehensively through 
empirical field studies, but are reasonably tractable through simulation.

The second use case also addressed an inherent aspect of hyperspectral cameras - the choice of grating - that could in principle be altered to potentially improve accuracy of a remotely sensed environmental parameter, here again FLH. The simulation was modified to mimic a camera incorporating a NIR-blazed grating, as opposed to the visible-wavelength grating that is customarily expected. Simulation results (Fig. 3-2 reveal what trade-offs arise for drone-based measurement of FLH when selecting between a 12-bit camera with a grating blazed for the visible spectrum, and an 8-bit camera with a grating blazed for the NIR spectrum. Here the simulation reveals that error in the FLH estimates will be similar between an 8-bit NIR-blazed camera system and a 12 bit VIS-blazed camera system, but otherwise this may be unclear from purely empirical observations. Such a simulation approach becomes even more valuable when a larger number of possible choices in camera parameters must be weighed.

The third use case examined choices in operational parameters, such as exposure time, that can have important consequences when imaging water-leaving radiances with small aerial drones over natural water bodies. Drones are especially valuable for studying aquatic systems that exhibit substantial heterogeneity on fine spatial scales, such as the marginal ice zone in polar oceans, aquaculture farms in coastal systems, and river outflow regions in estuaries. In such scenarios it may be desirable to cover a large area at the finest possible spatial resolution, which would require a low exposure time and a drone flying at maximum velocity. A user in this case will need to know the minimum exposure time required to obtain a desired accuracy in a given OAC of interest. This simulation tool explored this class of problem in the context of ocean areas covered with interspersed sea ice, quantifying the limitations on spatial resolution set by the actual spatial heterogeneity in the image (Fig. 3-4) as well as trade-offs between effective spatial resolution and accuracy in any given remotely sensed variable of interest (Fig. 3-5). From this analysis, a researcher desiring to image phytoplankton chlorophyll in the open water among $99 \%$ sea ice concentration would opt to reduce the target spatial resolution from $1.6 \mathrm{~m}$ to $0.8 \mathrm{~m}$ to be able to capture the $6 \%$ increase in the fractional area of the water, sacrificing accuracy in MAPD only by $8.9 \%$. This would represent a strategy to improve mapping for the presence of phytoplankton between floes at the expense of accuracy in estimates of phytoplankton biomass inferred from remotely sensed chlorophyll. Such insight is valuable to have before conducting an 
actual aerial camera deployment in the field.

The fourth use case examined an under-explored use of aerial hyperspectral cameras that may have significant applications in aquatic remote sensing. The simulation examined how the dynamic range (full-well capacity) of currently available spectral cameras limits the estimation accuracy for chlorophyll between ice floes in the earlier case of intermittent sea ice cover (Fig. 3-4). By quantifying this problem through simulation, optical designers can better develop engineering solution to this dynamic range limitation. The solution explored here leverages the phenomenon of multiple orders produced by the gratings that are often used to provide dispersion in line-scan cameras [Sigernes et al., 2018]. Gratings split light into multiple orders with different efficiencies and spectral resolutions, and generally the lower efficiency higher orders are engineered out of the final measurement, effectively discarded as wasted photons. The second order spectrum, which has lower efficiency, could be used to image the much brighter sea ice algae, while the higher efficiency first order spectrum could image the chlorophyll in phytoplankton between floes. With this approach the overall camera exposure time could be increased to optimize estimation accuracy of phytoplankton chlorophyll, leveraging the first order spectrum where the sea ice algal signature saturates, while retaining the second order spectrum to estimate sea ice algal chlorophyll without saturation. Similar situations with high dynamic ranges can reasonably be expected in many other drone-based aquatic imaging scenarios such as in surface-ocean kelp farms or in complex river outflows.

This framework is not comprehensive for all potential aspects of hyperspectral imaging, and camera artifacts not examined here include keystone Mouroulis and Green, 2018, smile, stray light, and environmental fluctuations due to variability in insolation. Keystone and smile introduce distortions that can presumably be corrected for via calibration, but stray light is a potentially deleterious issue in scenarios where radiances are dramatically different between adjacent pixels, as would be seen from aquatic bodies with substantial surface glint on nearby wave facets. Here, stray light from glint pixels could be incident on adjacent pixels on the camera's FPA, requiring correction. Surface glint of reflected sunlight or skylight is a real-world source of 'noise'. Another aspect of these simulations that merits mention is the use of radiative transfer models (e.g., Hydrolight) to generate synthetic spectra for the different use cases. This can be valuable when no real-world observations are available, but synthetic radiance data may be incomplete as in the case of Hydrolight runs using the 
provided absorption curves, which stop at $700 \mathrm{~nm}$ and are interpolated into the NIR. In these four exploratory use cases this limitation was not a concern, but it may be important in scenarios where OACs of interest require exact measurement of the NIR O'Shea et al. 2020 .

\subsection{Conclusion}

A simulation framework can provide valuable information for exploring quantitative relationships between hardware properties, deployment options, and OACs of interest in dronebased aerial monitoring of aquatic systems. Although it is examined here in the context of an idealized hyperspectral camera, this framework can be readily extended to assessment of multispectral camera systems after slight modifications [O'Shea, 2020]. The use cases presented here involved assessments of a) existing camera systems with predetermined choices in particular parameters, b) potential modifications to existing camera designs, and c) potential new uses of existing hyperspectral camera systems. All three modes of application can advance the use of spectral imaging of natural water bodies for research and monitoring purposes. 


\title{
Chapter 4
}

\section{Spectral super-resolution via spectral shift in spatially oversampled images: Considerations for sub-meter ocean color remote sensing}

\begin{abstract}
Achieving a fine spatial resolution over the dark ocean comes at the cost of spectral resolution to maintain the required signal-to-noise ratio for ocean color remote sensing applications. Homogeneous oceanic regions oversampled by grating-based imaging spectrometers contain non-redundant spectral information, due to a spectral shift along the spatial axis, which can be leveraged to increase accuracy of the spectral frequency representation at values higher than the pixel-limited Nyquist frequency. Specifically, we demonstrate that an imager with $1.8 \mathrm{~nm}$ of spectral shift, $1.7 \mathrm{~nm}$ of spectral blur, and a $2.1 \mathrm{~nm}$ pixel sampling interval can estimate the spectral content produced by a point spectrometer with $0.2 \mathrm{~nm}$ pixel sampling interval and $0.7 \mathrm{~nm}$ of spectral blur with $5.3 \%$ error, as opposed to $34.8 \%$ without spectral shift, by using support vector regressions (SVRs). When properly trained, this technique even provides increased spectral resolution on data suffering from high Gaussian and non-Gaussian noise sources typical of ocean color scenarios. Finally, the super-resolution technique does not require the full image width, and can provide a significant accuracy increase even if only half of the spatial data is input into the SVR. While this technique increases the accuracy of frequency content slightly beyond the Nyquist on laboratory generated ocean color signals, further research must be conducted to increase generalizability of the technique.
\end{abstract}

\subsection{Introduction}

Certain ocean color remote sensing applications, such as imaging the interface between a river out-flowing into the coastal ocean, require a sub-meter spatial resolution to separate the spectral content at discrete interfaces. However, the sub-meter spatial resolution required 
to separate the distinct regions will spatially over-sample in homogeneous regions, such as the distinct water bodies on either side of the interface. In ocean color remote sensing these over-sampled regions are typically binned for $\mathrm{km}$ scale measurements from satellites Carder et al., 1993, Hu et al., 2012, Davis et al., 2002, to increase the signal-to-noise ratio (SNR) to sufficient levels for the specific task, or spatially filtered for sub-meter applications from drones [Totsuka et al., 2019; Kwon et al., 2020], to reduce the impact of fluctuations in the surface reflected light. An alternative image processing approach could leverage sub-pixel shifts in the measured spectrum within the homogeneous regions, to increase the spectral resolution in post-processing. This additional spectral information could then be used for spectral inversion tasks whose accuracy is dependent upon the number of input wavelengths, such as phytoplankton functional type identification Aiken et al., 2014; Lubac et al., 2008, or tasks that require spectral resolutions beyond the commercially available values, such as satellite ocean color validation [Zibordi et al., 2017]. The sub-pixel shifts typical in grating based imaging spectrometer data are the result of optical aberrations, such as the spectral curvature effect ('smile') Mouroulis et al., 2000; Fisher and Antomades, 1998; Davis et al., 2002 or focal plane array misalignment Meola, 2018, D'Odorico et al., 2010. No matter the source, machine learning algorithms may be able to leverage the non-redundant spectral information contained within the sub-pixel spectral shifts to increase the spectral resolution beyond the hardware limit. While applying the machine learning algorithm to terrestrial or industrial remote sensing tasks would be relatively straightforward due to their low noise and high intensity, ocean color remote sensing requires further introspection as the low signal-tonoise ratio (SNR) and high levels of environmental noise (e.g., surface reflected light) may limit the efficacy of such an algorithm [O'Shea et al., 2020; O'Shea and Laney, 2020].

This paper demonstrates the use of support vector regressions (SVRs) to leverage the sub-pixel shifts in homogeneous data to achieve spectral super-resolution beyond the pixellimited Nyquist frequency. The paper then addresses the potential limitations of applying this spectral super-resolution technique for sub-meter ocean color data, due to its low optical SNR, high environmental noise, limited spatial range, and specific spectral content. 


\subsection{State-of-the-art spectral super-resolution techniques}

Broadly, spatial super-resolution (SR) aims to use additional information captured within low resolution images to increase the spatial resolution beyond the nominal system limits set by either the pixel-limited sampling interval or point spread function (PSF) Yang and Huang, 2018]. The additional, non-redundant, information can be from sub-pixel shifts between multiple low-resolution (LR) images of the same scene (which vary temporally or spatially) Carles et al., 2014; Tsai and Huang, 1984; Kim et al., 1990, a priori statistical information (i.e., via learned dictionaries) for single image super-resolution Yang and Huang, 2018], or knowledge of the systems physics. The initial theoretical considerations of using additional images to increase the spatial resolution occurred in 1984 [Tsai and Huang, 1984]. Since this seminal paper, significant work has been done to achieve spatial super-resolution through a variety of different methodologies for numerous applications ranging from medical to industrial applications Abd El-Samie et al., 2012; Yang and Huang, 2018.

Within the spectral remote sensing domain, SR techniques have mainly been used to increase the spatial resolution of the spectral images. Two of the main categories of superresolution techniques that have been used include: single image SR leveraging dictionarybased learning, which learns correlations between low resolution and high resolution data [Liebel and Körner, 2016], and sensor fusion between a low spatial but high spectral resolution imager and a high spatial but low spectral resolution imager Abd El-Samie et al. 2012, Pan and Shen, 2019; Fang et al., 2018; Ma et al., 2014]. In the dictionary-based learning technique, correlations between the low spatial resolution data and the high spatial resolution data are learned from a prior dataset. A main limitation of this approach is that the correlations must stay the same in any future images (i.e., the technique will not provide high accuracy if the subject of the images departs from the learned statistics). In the sensor fusion technique, correlations between the low spectral resolution and high spectral resolution data are made, assuming sparsity, to achieve a high spectral resolution and spatial resolution output Abd El-Samie et al., 2012. In one example, the spectral information from a multispectral camera was fused with a high spatial resolution RGB camera, to achieve a high spatial and spectral resolution image Pan and Shen, 2019. While increasing the spatial resolution is useful in heterogeneous regions, these techniques would not provide meaningful information in sub-meter sampled ocean color scenarios where the spatial resolution is only 
required to separate distinct regions.

Researchers have focused significantly less on spectral super-resolution, which overcomes the spectral resolution limits of the available hardware by leveraging non-redundant spectral information contained within the images. Again a large focus of single image spectral SR has been on dictionary-based techniques. Just like in the spatial resolution case, in coupled dictionary learning correlations between the low spectral resolution data and high spectral resolution data are learned from a sample dataset, and then applied to the low spectral resolution data to achieve high spectral resolution estimates. A coupled dictionary learning approach was successfully applied to terrestrial remote sensing data by learning correlations between satellite sensors with different spectral resolutions [Fotiadou et al., 2019]. In addition to being proven on terrestrial targets, dictionary based-learning has also been applied to ocean color remote sensing, where the spectral resolution was increased by assuming sparsity of the components Charles et al. 2014. While most research has focused on using dictionary-based learning to increase the spectral resolution of preexisting sensors, the methods can also be used to optimize the bands required for future design, to obtain the best estimates of the hyperspectral data Gewali et al., 2019. A large limitation of these spectral correlation approaches is that they rely on pre-learned information, and may not transfer well to novel datasets.

A promising new area of spectral SR leverages optical information contained within each image due to the imagers design, instead of relying on correlations learned from prior datasets; thus enabling generalization to a broader range of input signals outside of the training sets statistics. A widely utilized technique in this domain is the introduction of a coded aperture to introduce sparsity into the imaging system, which can then be leveraged to increase the spectral resolution Galvis et al. 2015. Another spectral SR technique overcame the pixel-limited Nyquist frequency by leveraging the Moiré effect; and ended up increasing the spectral resolution by a factor of 10 Konishi et al. 2016. In yet another technique, the spectral resolution achievable by a Fourier imaging spectrometer overcame the Nyquistlimited value by tilting the axis relative to the imaged interferogram on the focal plane array, so that the imaged fringe's were slightly offset on a pixel-by-pixel basis Watanabe and Furukawa, 2018, thus introducing sub-pixel shift. This same idea of shifting the focalplane array was also applied to a grating based imaging spectrometer to increase the spectral resolution beyond the hardware limit in a seminal study by Kitano et al. Kitano et al. 
2020]. Overall, these techniques that leverage non-redundant information within the image can theoretically generalize to a broader range of input signals as they increase the fidelity of the data by leveraging information added by the optical system, instead of correlations learned from prior data.

\subsection{Application to Sub-meter Ocean Color Remote Sensing: Motivation And Limitations}

Specific regions of the ocean require sub-meter spatial resolution to sense ocean color features, but end up oversampling homogeneous sections of the ocean. Example regions include sensing between cracks in the sea ice [O'Shea and Laney, 2020], mapping algal mats, or imaging the interface between water bodies, such as a river out-flowing into the coastal ocean (Fig. 4-1). In this last example, the interface requires sub-meter data to separate distinct regions and potentially resolve mixing, but either side of the interface may be over-sampled. As previously mentioned, the pixels comprising the over-sampled regions are typically either binned to increase the signal-to-noise ratio for sensitive ocean color tasks Carder et al., 1993, $\mathrm{Hu}$ et al. 2012, Davis et al., 2002 or spatially filtered to reduce the impact of fluctuations in surface reflected light [Totsuka et al., 2019, Kwon et al., 2020]. However, these additional filtering steps do not substantially increase the fidelity of the data if the SNR is non-limiting and glint has already been spectrally corrected [O'Shea et al., 2020]. These additional spatial measurements could instead be used to increase the spectral resolution for specific tasks which are either ill-posed, such as phytoplankton species identification (phytoplankton functional type identification), or have high spectral resolution requirements, such as satellite ocean color validation [Zibordi et al., 2017].

Unfortunately, there are a few optical characteristic of sub-meter ocean color data which may limit the efficacy of a spectral super-resolution algorithm. First, hyperspectral measurements of the coastal ocean can have low signal-to-noise ratios, ranging from 10-70 in near ideal conditions O'Shea et al., 2020, Honkavaara et al., 2013, but require high SNRs for high accuracy. The variations in the SNR could lead to poor performance of the SR approach, as increased noise can non-linearly effect the accuracy of spectral SR techniques Sezer and Altunbasak, 2018]. Second, light reflected off the surface of the ocean on either side of the interface breaks the homogeneous assumption required for the proposed 
algorithm. Capillary waves induce $\mathrm{cm}$ scale fluctuation in the reflection coefficient, which can vary by $35 \%$ to $100 \%$ depending on wind and viewing conditions [Carrizo et al. 2019]. While these fluctuations are largely corrected by spectral algorithms, there still exist slight offsets due to imperfect correction of the surface reflected light ['Shea et al., 2020]. These imperfections may limit the homogeneity assumptions of the radiance from each side of the interface. These two characteristics of ocean color data, the low signal-to-noise ratio and the imperfect glint correction, limit the homogeneity assumption and therefore the efficacy of spectral super-resolution approaches, if the SR techniques are not appropriately trained on data containing these optical characteristics.

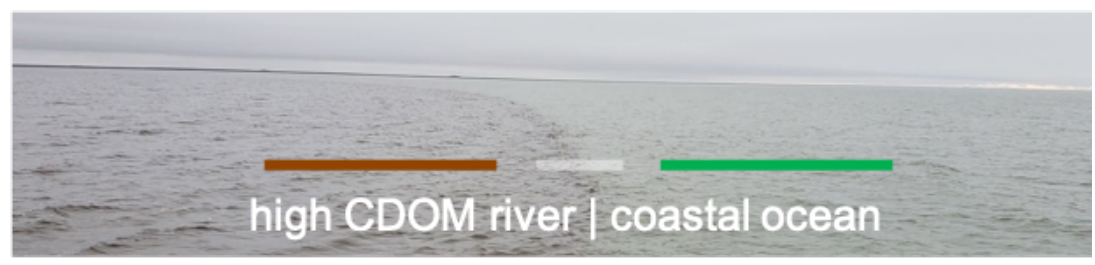

Figure 4-1: The interface between a river with high colored dissolved organic matter $(\mathrm{CDOM})$ and the coastal ocean, dominantly containing green algae, in northern Alaska. High spatial resolution images are required to sense the interface (grey bar), while the homogeneous waters in the high CDOM river (brown bar, left) and coastal ocean waters (green bar, right) are over-sampled. Photo credit: Luka Ćatipović.

\subsection{Methods}

\subsubsection{Sources of Sub-Pixel Spectral Shift in Grating Based Imaging Spec- trometers}

As previously mentioned, sub-pixel spectral shift can occur in images taken by grating-based imaging spectrometers from either optical misalignment Meola, 2018, D'Odorico et al., 2010], for example a tilted focal plane array relative to the optical axis of the system, or the spectral curvature effect ('smile') [Mouroulis et al. 2000]. An ideal imaging system without optical aberrations imaging a homogeneous target will produce monochromatic images of the slit that maintain the same vertical position as a function of the spatial domain (Fig. $4-2$ A). If the focal plane array is tilted relative to the optical axis, then each spatial pixel within a given row will have slightly different spectral composition due to spectral shift along the spatial axis (Fig. 4-2, B). In a non-ideal imager which suffers from the spectral 
curvature effect, the monochromatic image of the slit produced at each wavelength varies as a function of the spatial axis (Fig. 4-2, C) [Mouroulis et al., 2000. When sampled by an on-axis (or even off-axis) focal plane array (Fig. 4-2, D) the spectral content of the pixels in each row then contains sub-pixel spectral shifts across the spatial axis. No matter the cause of spectral shift, when imaging a homogeneous target these sub-pixel shifts theoretically provide the non-redundant information required for spectral super-resolution.

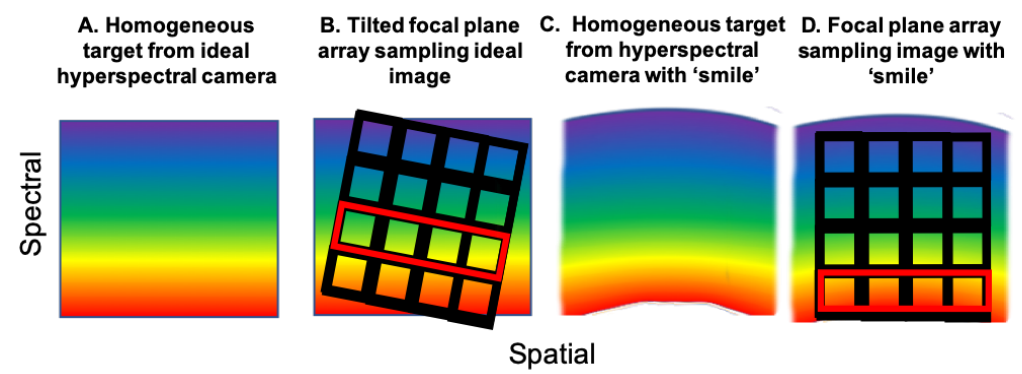

Figure 4-2: In an ideal image of a homogeneous target made from a imaging spectrometer, the vertical position of any individual spectra does not vary as a function of spatial location (A). The sub-pixel spectral shift required for super-resolution can be induced in ideal imagers by tilting the focal plane array (FPA) relative to the optical axis (B, red box), or can occur in non-ideal imagers suffering from the spectral curvature effect ('smile', C) when measured by an on-axis FPA (D, red box).

\subsubsection{Dataset collection: Images with sub-pixel shift and associated truth spectra}

Training a machine learning approach for spectral super-resolution from homogeneous images suffering from a wide array of noise sources requires a large dataset of coarse spectral resolution images with sub-pixel spectral shift and 'truth' spectra measured at a finer spectral resolutions. The optical setup to collect such a dataset requires a tunable spectral output that can be simultaneously imaged by the truth and test instruments (Fig. 4-3). The setup begins with a $20 \mathrm{~W}$ tungsten halogen light that provides a broad spectrum as input to a digital monochromator (Fig. 4-3). The digital monochromator rotates through a variety of wavelength passbands for random (uncorrelated) amounts of time to generate the test spectra. The wavelength spacing between stopping points is $1 \mathrm{~nm}$, plus or minus a random (uncorrelated) amount (max of $0.625 \mathrm{~nm}$ ) at each central wavelength. The output spectra propagates through a Thorlabs 50 um bifurcated fiber, which is a fiber with a single SMA connector with two 50 um fibers that splits into two SMA connectors each with 
their own output. The bifurcated fiber allows nearly identical light to be output to two different instruments. The two instruments include the 'truth' Ocean Optics Spectrometer, which has a $0.2 \mathrm{~nm}$ sampling interval and $0.7 \mathrm{~nm}$ of spectral blur [full-width half-maximum (FWHM)], and the 'test' Resonon Pika L imaging spectrometer (detailed below). These two instruments are hardware triggered so that they measure the same input signal.

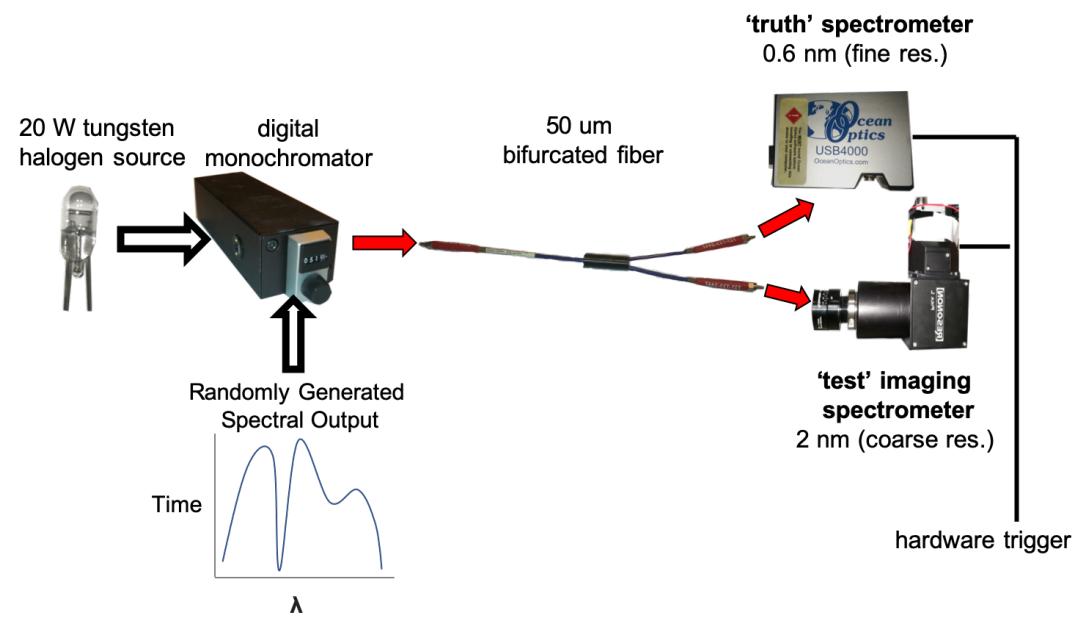

Figure 4-3: A 20 Watt Tungsten Halogen source provides white light as input to a digital monochromator. The monochromator shifts wavelengths after random amounts of time, to generate a randomly generated spectral output. The light passes through a 50 micrometer bifurcated fiber, which allows the same input light to pass through a 'truth' spectrometer with fine spectral resolution (0.7 nm FWHM of spectral blur, $0.2 \mathrm{~nm}$ sampling) and a 'test' imaging spectrometer with coarse (1.7 nm FWHM of spectral blur, $2.1 \mathrm{~nm}$ sampling) spectral resolution. The instruments are hardware triggered so that the measure the same input signal.

The Resonon Pika L imaging spectrometer was chosen as the test instrument as it has already been proven for ocean color measurement [O'Shea et al. 2020]. The imaging spectrometer's spectral resolution is limited by the slit-width, which imposes $1.7 \mathrm{~nm}$ (FWHM) of spectral blur, and by the sampling pixels to $1.05 \mathrm{~nm}$, though this increases to $2.1 \mathrm{~nm}$ when binned by 2 . The spectrometer contains 1.7 spectral pixels (corresponding to roughly 1.8 $\mathrm{nm}$ of spectral shift) along the spatial axis (Fig. 4-4). The spectral shift was estimated by fitting a Gaussian at each spatial location to the spectral output from the monochromator when it passes a single wavelength (repeated for 25 iterations). Then the mean spectral pixel of the Gaussian fit can be plotted as a function of the spatial pixels, to determine the shape of the spectral shift. This method produces a slightly quadratic shape, characteristic of smile, with a linear trend, characteristic of an offset focal plane array relative to the optical 
axis. Overall, it is clear that there is a shift in the spectral pixel that measures any given wavelength across the focal plane array, which can provide the non-redundant information necessary for spectral super-resolution.

The total training and testing dataset consists of 977 images (from the 'test' imaging spectrometer) with matching 'truth' spectra (from the 'truth' spectrometer). two third's (651) of this dataset is used for training, one sixth (163) for validation, and one sixth (163) is left for testing. To compare between images with spectral shift (henceforth referred to as SS), and without spectral shift (i.e., no spectral shift, NSS), a small subset (11) of the pixels in a local region are used as data without sub-pixel spectral shift. The same machine learning techniques applied to images containing sub-pixel spectral shift can be applied to these images lacking spectral shift, so that the benefit of the machine learning algorithms can be compared with and without sub-pixel spectral shift.

Three testing sets were generated to test the ability of the super-resolution technique to generalize to images with statistics outside of the training sets. The first set was laboratorygenerated band-pass/band-stop signals, the second was laboratory-generated ocean color measurements, and the third consisted of two separate real-world filters. Twenty eight bandpass/band-stop signals were generated, they were comprised of four sets with $\sim 1,2,3$, and $4 \mathrm{~nm}$ FWHM's and each set has 7 different relative intensity levels in the pass/stop bands $(\sim+/-0,25,50,75 \%)$. Although intended to have those specific intensities, the spectral generation hardware and optics limited the exact intensity reached in each case. For the ocean color dataset, data from the Marine Optical Buoy (MOBY) was used Clark et al. 2003. MOBY was chosen as it was of the only instruments that had readily available ocean color data with sufficient spectral resolution $(<1 \mathrm{~nm})$ for testing the proposed spectral super-resolution algorithm on the available imaging spectrometer. Four of the sharpest spectral features were chosen from two different MOBY datasets, one typical of the nearby oligotrophic (very low algal content) waters (2020061823D) and one with higher intensity greens characteristic of low to medium algal content (2002101920D) Flora. For the realworld filter measurements, two different Thorlabs Filters were used for their relatively high spectral variations in the red, a $950 \mathrm{~nm}$ cut-off short pass filter (FES0950), and a $1000 \mathrm{~nm}$ cut-off short pass filter (FES1000). Each filter was measured twice, first straight on and then at a slight angle, as the spectral transmission efficiency changes as a function of view-angle. Overall, the combination of these testing sets allow for testing on a wide range of input 


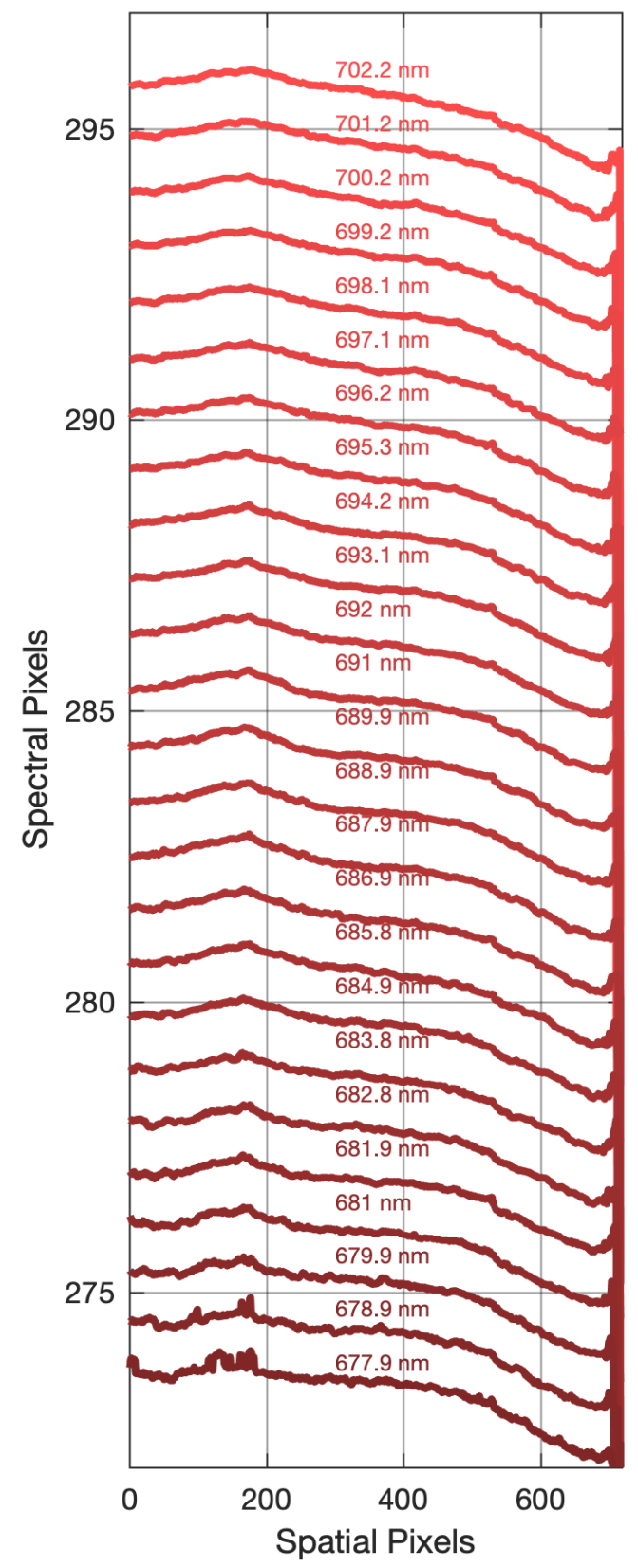

Figure 4-4: The spectral pixel that measures a monochromatic light source (e.g. $702.2 \mathrm{~nm}$, top curve) is plotted for each spatial location, for 25 different central wavelengths. The spatial dependence, which is similar in shape to a quadratic characteristic of smile and a linear trend from a shifted focal plane array, holds across the wavelength range. The technique fails at low SNRs, which occur above spatial pixel 700 (right side). The median change between the highest spectral wavelength and the lowest spectral wavelength (from spatial pixel 1-700) is 1.7 pixels, which corresponds to $\sim 1.8 \mathrm{~nm}$ of spectral change in the reported wavelength over the spatial domain. 
signals outside of the training statistics.

All of the truth OOS measurements, including the training, validation, testing, and the three out-of-training testing sets were normalized by the median of 37 constant sweeps of the monochromator made in-between measurements of the three out-of-training testing sets. The spectral content of the tungsten halogen source varies as a function of temperature, so these normalizing values will not perfectly normalize the training dataset. This normalization factor is intended to remove changes in the spectral efficiency of the optical system, so that the SVR does not learn that constant high frequency spectral content. Theoretically, a machine learning approach could learn the normalizing factor through training.

\subsubsection{Machine Learning Optimization}

A support vector regression (SVR) is trained for each spectral output in the same manner. Five-fold cross-validation is used to determine the best hyperparameters (box constraint, kernel scale, and epsilon) for training. The same training set used during cross-validation is then trained with the optimized hyperparameters. The testing error is reported for the iteration with the lowest validation error. Median absolute percent difference (MAPD) is the error metric used to compare between the estimated value, produced by the SVR using the course resolution imaging spectrometer data, and 'truth' value measured by the Ocean Optics spectrometer [Eq. 4.1, as it is insensitive to outliers [Cui et al., 2013.

$$
M A P D=100 \% *(\exp [\text { median } \mid \ln (\text { estimated } / \text { truth }) \mid]-1)
$$

\subsubsection{Setting the Signal-to-Noise Ratio with Gaussian Noise}

The signal-to-noise ratio in red sections of ocean color data can be on the order of 10 O'Shea et al., 2020], or even lower if the spatial and or spectral resolution limits of the imager are pushed. Gaussian noise is added to the images to reduce the SNR to 10, to determine how the accurately the SVRs can recover the super-resolved signal despite the low SNR. The SNR is reduced based on assuming the underlying signal is shot-noise limited (which is not a perfect assumption as the amplifier stage, which applies an analog gain of 16, adds amplifier noise). The equation used to set the standard deviation of the Gaussian noise (SD) 
that is added on top of the signal is:

$$
S D=\left(\frac{\text { Electrons }^{2}}{S_{N R_{\text {desired }}}^{2}}-\text { Electrons }\right) 0.5
$$

Where Electrons is the number of electrons at each pixel and $\mathrm{SNR}_{\text {desired }}$ is the desired signal-to-noise ratio. The electrons are calculated by multiplying the digital numbers by their normal conversion factor of 7.8942 O'Shea et al. 2020 and then dividing that by 6.3 (to represent the impact of the gain of 16 applied to the input signal). This equation enables the entire image to have the same SNR by compensating for variations in the inherent noise introduced due to a lower intensity on the edges of the image from vignetting.

\subsubsection{Simulating Residual Glint on the Laboratory Collected Dataset}

The imperfect glint removal of sub-meter data results in offsets (Fig. 4-5, top) that can be assumed to be spectrally flat in a local $(\sim 20 \mathrm{~nm})$ region. While the distribution of these offsets are sometimes near Gaussian, they are more often tailed due to over- and undercorrection of sections containing capillary wave induced fluctuations in the surface reflected light (Fig. 4-5, bottom). The skewness and kurtosis of these distributions were only weakly correlated with wind-speed and direction measured from a mast $3 \mathrm{~km}$ away. While these measurements show that the distributions have a generally weak correlation with average wind-speed, the imperfect spatial and temporal alignment with the measured distributions does not provide accurate estimation of the correlation with local gusts of wind.

The tailed Gaussian distribution may hinder the glint correction process, as the distribution inherently breaks the required heterogeneity assumption. Distributions of the residual glint offsets were created by taking the median value at a high SNR wavelength $(505 \mathrm{~nm})$, subtracting it from each image, and then calculating the percent offset of each spatial pixel. These distributions are then sampled, with a random $+/-<10 \%$ offset applied to keep the algorithm from learning the exact underlying distribution, and applied to the collected training data. Of the $\sim 350$ images collected during the deployment, the distributions are sub-sampled with $4 / 6$ used for training, $1 / 6$ for validation, and $1 / 6$ for testing. Keeping the distributions separate for the training, validation, and testing sets determines if the spectral SR algorithm will generalize to new residual glint offset distributions. While the sub-meter ocean color data often has more spatial form, this would only be additional information for 
the machine learning algorithm to obtain. In other words, the error produced by these flat offsets serves as an upper bound to the error that would be expected in the real-world glint corrected data.

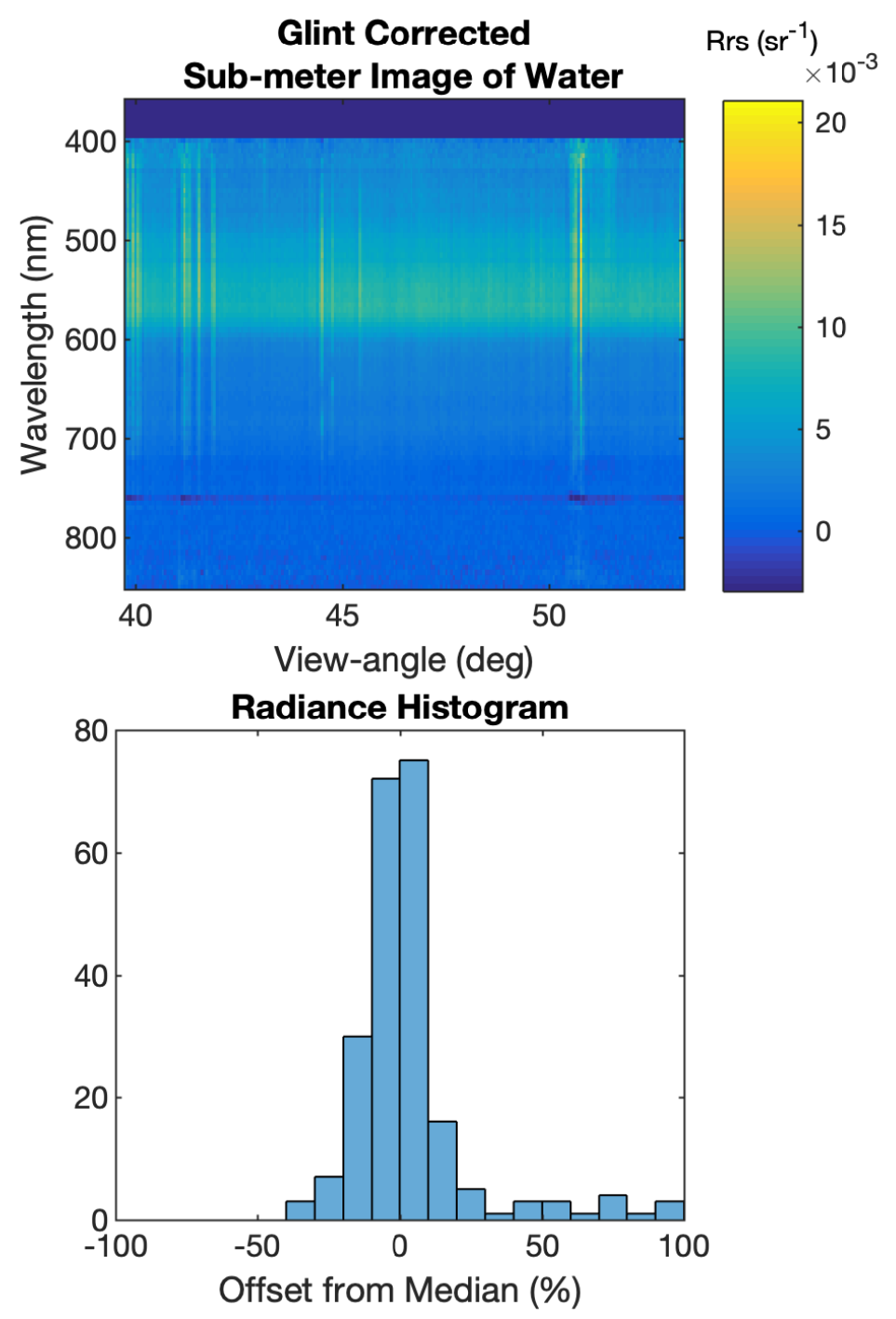

Figure 4-5: An example cm scale hyperspectral image of homogeneous oceanic waters suffering from residual glint correction errors (top, spectrally flat offsets near 43 and 51 degrees) O'Shea et al., 2020. The distribution of radiance at $505 \mathrm{~nm}$ normalized by the median value is non-Gaussian and includes significant offsets (bottom). 


\subsection{Results}

\subsubsection{Leveraging sub-pixel spectral shift in homogeneous images for in- creased spectral resolution}

As previously noted, two sets of SVRs are trained on different course spectral resolution imaging spectrometer datasets, one with spectral shift (SS) and one without spectral shift (NSS), to predict the fine spectral resolution output by an Ocean Optics point spectrometer. The first dataset is full spatial dimension hyperspectral images of homogeneous targets, which have $1.8 \mathrm{~nm}$ of spectral shift (Fig. 4-4). The comparison dataset consists of only 11 local spectra from within the original image, and so does not have significant spectral shift. The SS SVRs achieve much higher training and validation accuracy than the NSS SVRs. An example training curve for a single spectral output trained on data with SS (solid black line, Fig. 4-6), converges within 15,000 iterations and generalizes well on the validation set (solid red line, Fig. 4-6), though there is significant overfitting on the training data. The validation set for data with SS (solid red line, Fig. 4-6) is able to achieve much lower error than even the training data without spectral shift (black dashed curve, Fig. 4-6). Similar results hold for all 53 of the output spectra, which makeup the output vector. Overall, the dataset which includes spectral shift is able to clearly increase the estimation accuracy of the intensity measured by a separate instrument at resolutions beneath the pixel limited resolutions on data with similar statistics.

Visually, example output vectorized training and testing data demonstrate the increased accuracy of SS SVRs relative to NSS SVRs (Fig. 4-7). The output vectors produced by the SS SVRs (Fig. 4-7, red line) match the Ocean Optics spectrometer 'truth' (Fig. 4-7, black line) in both the training and testing subsets. The SS SVRs predicted spectrum matches both the training and testing data across a wide range of frequencies. Visually, the NSS SVRs (Fig. 4-7, teal line) are able to match the low and mid frequency fluctuations in both the training and testing data, but don't match the intensity of the truth data (Fig. 4-7, black line). Both the SS and NSS better represent the medium frequency fluctuations than the median of the NSS camera measurements that have been re-scaled (RS) to match the normalized intensity measurements and spline interpolated between pixels (Fig. 4-7. blue line, asterisks are pixel centers). While the RS data accurately captures low frequency fluctuations, it severely underestimates the high frequency portions of the 'truth' signal. 


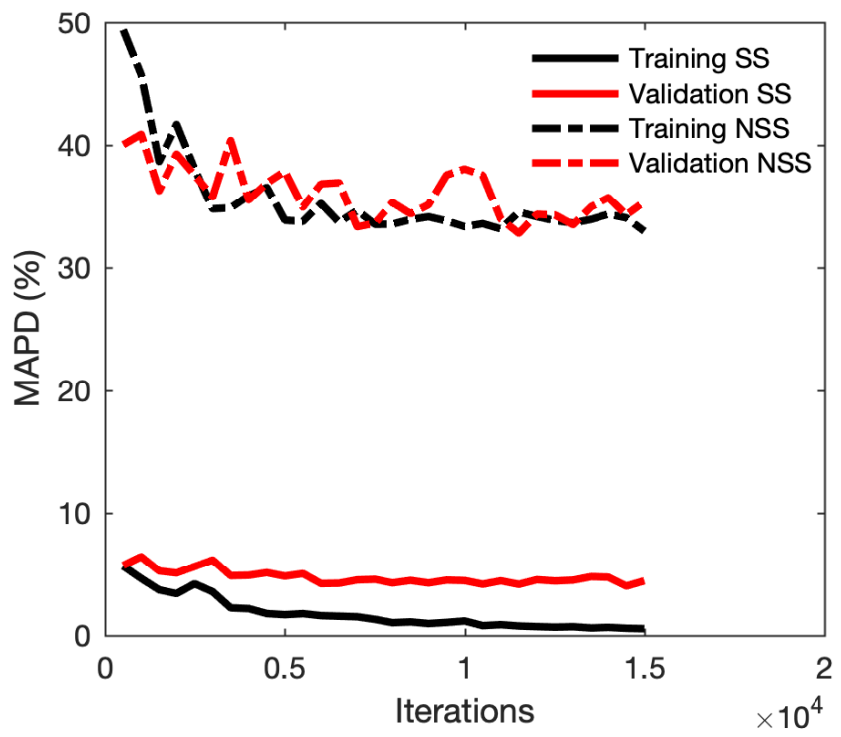

Figure 4-6: Example training and validation MAPD between the spectrometer measured intensity and the SVR estimated intensity from homogeneous images with $1.8 \mathrm{~nm}$ of spectral shift (SS) and on a SVR trained on data without spectral shift (NSS) for one spectral output. Both SS and NSS SVRs converge within 15,000 iterations. The SS SVR achieves a much higher validation accuracy $(\sim 4 \%)$ than the NSS SVR $(\sim 33 \%)$.

Overall, visually the predicted spectral output by the SS SVRs matches both low and high frequency data in these two example training and testing images better than the NSS SVRs or RS camera measurements.

The visual analysis of the relative frequency dependent accuracy of the different prediction methods for these two examples (Fig. 4-7) are supported by a more rigorous Fourier analysis of the output spectra of the testing subset (Fig. 4-8). For an example testing spectra, the SS SVRs best match the frequency content of the testing data until about 1 $\mathrm{nm}^{-1}$, which is past the pixel-limited Nyquist frequency $\left(4.2^{-1} \mathrm{~nm}^{-1}\right.$, for a $2.1 \mathrm{~nm}$ bandwidth pixel, Fig. 4-8, top). The NSS SVRs are able to match the frequency content until just before the Nyquist (Fig. 4-8, top, teal curve), and then do not match the spectral content again until $1 \mathrm{~nm}^{-1}$, which is approaching the 1st standard deviation of the spectral PSF of the spectrometer (Fig. 4-8, top, light grey dot dashed line). Spectral frequencies beyond this limit would be heavily attenuated in the imaging spectrometer images. The re-scaled Pika L measurements (Fig. 4-8, blue) only represent the lowest frequency data well. More generally, by taking the median absolute percent difference between the single sided power spectrum of the OOS Truth and the SS, NSS, and RS predictions over all of the test spectra 


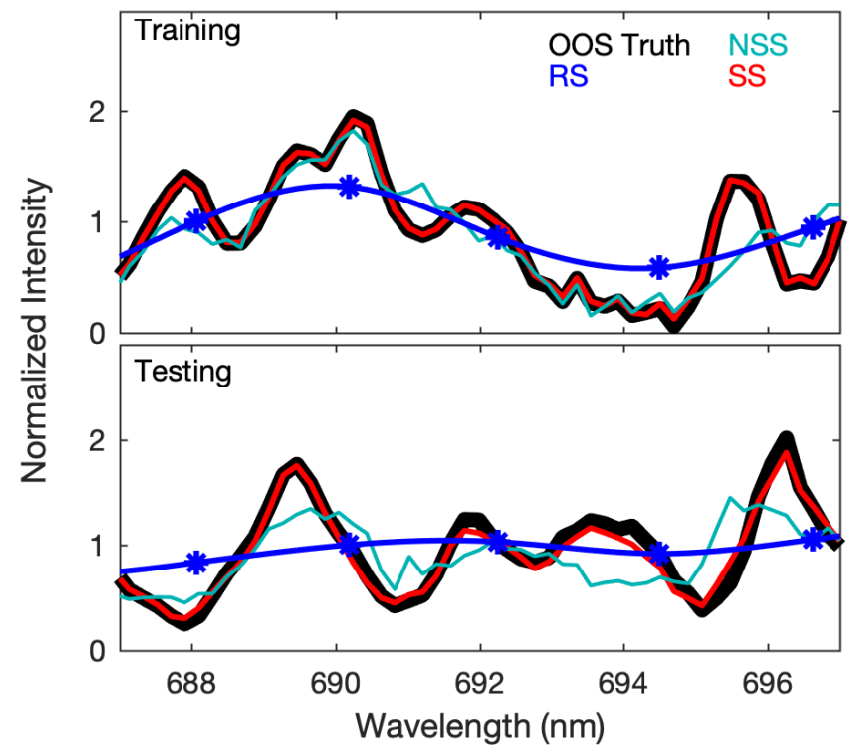

Figure 4-7: Example predictions for training and testing datasets. The SVRs trained on data including spectral shift (SS) match the low and high frequency fluctuations in the normalized intensity measurements from the Ocean Optics spectrometer (OOS Truth) values (black) better than either the SVRs trained on data without spectral shift (NSS, teal) or the median of the NSS data re-scaled to the normalized intensity values (RS, blue, asterisks are pixel center wavelengths).

shows that the SS SVRs actually achieves higher accuracy across the entire spectral domain (0-2.5 $\left.\mathrm{nm}^{-1}\right)$ (Fig. 4-8, bottom panel). Overall, the SS SVRs provide the most accurate frequency representation across the spectral domain on testing data with similar statistics as the training data.

\subsubsection{Generalization of SVRs for spectral super-resolution: ocean color signal, band-pass/band-stop, and real world signals}

Visually, the SS SVRs match low and mid frequency content in the laboratory simulated band-pass/band-stop signals (BP/BS, Fig. 4-9, A-D) and ocean color data (OC, Fig. 4-9. E-J), which have different statistics than the training and testing data. The SS SVRs better represent the location and intensity of the peak band-pass (BP) and band-stop (BS) signals (Fig. 4-9, A-D). However, the SS SVR predicted spectra suffers from significantly more high frequency noise than the re-scaled $(\mathrm{RS})$ camera measurements. The RS measurements are unable to match the intensity of the peaks or troughs, since they have sampling intervals that are wider than the underlying signal's fluctuations. The NSS SVR reconstruction does a very poor job recovering the Ocean Optics spectrometer 'truth' signal, even at relatively 

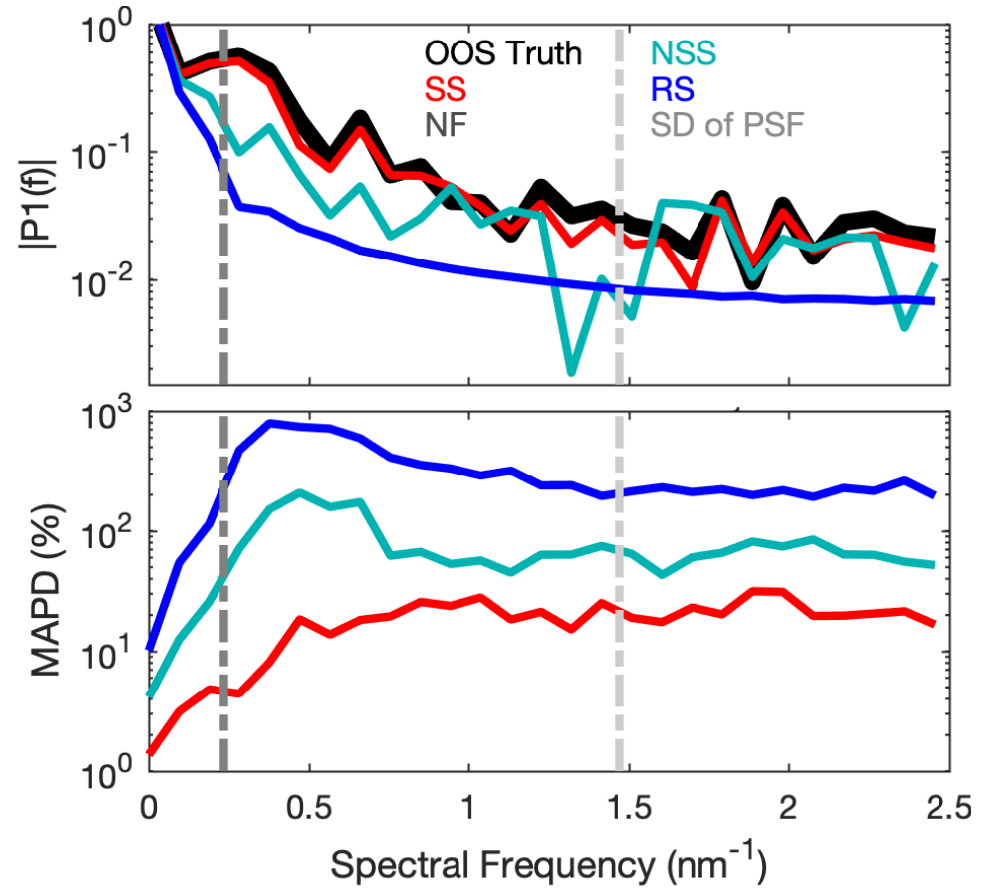

Figure 4-8: The single sided power spectrum of a prediction from the testing set using the SS SVRs (red, top panel) matches the truth data (black) across the spatial frequency domain, even past the pixel limited Nyquist frequency (NF, dark grey bars). The NSS SVRs (teal) match the frequency content until just before the Nyquist frequency, while the re-scaled (RS) raw pixel data (blue) only matches the spectral content at the lowest spatial frequencies. The median absolute percent difference between spectral content of the SS, NSS, and RS predictions and the OOS Truth values over the entire testing set demonstrate the benefit of the SS at all spectral frequencies (bottom panel). 
low frequencies. The same frequency dependence holds for the SS SVR predictions on the laboratory generated ocean color (OC) data as well. In most of the ocean color data with higher intensity drops (Fig. 4-9, E, F, G, H, J, K, and L) the highest intensity feature is well represented. However, in the data with lower intensity offsets (Fig. 4-9, I) the feature of interest is poorly captured. The benefits are particularly notable when the RS camera measurements almost completely miss the underlying feature (Fig. 4-9, K, 691-693 nm). The same technique failed to produce realistic mid to high frequency representation in all 4 real-world filter measurements (Fig. 4-9. M-P, where $\mathrm{M}$ and $\mathrm{N}$ are the FES0950 and $\mathrm{O}$ and $\mathrm{P}$ are the FES1000), the reconstructions were particularly bad when the filter was angled relative to the optical axis (Fig. 4-9, N and P). Overall, the SS SVR predicted spectra visually match low and medium frequency fluctuations in BP and OC data with higher accuracy than the NSS or RS techniques, but at the cost of significant high frequency noise.
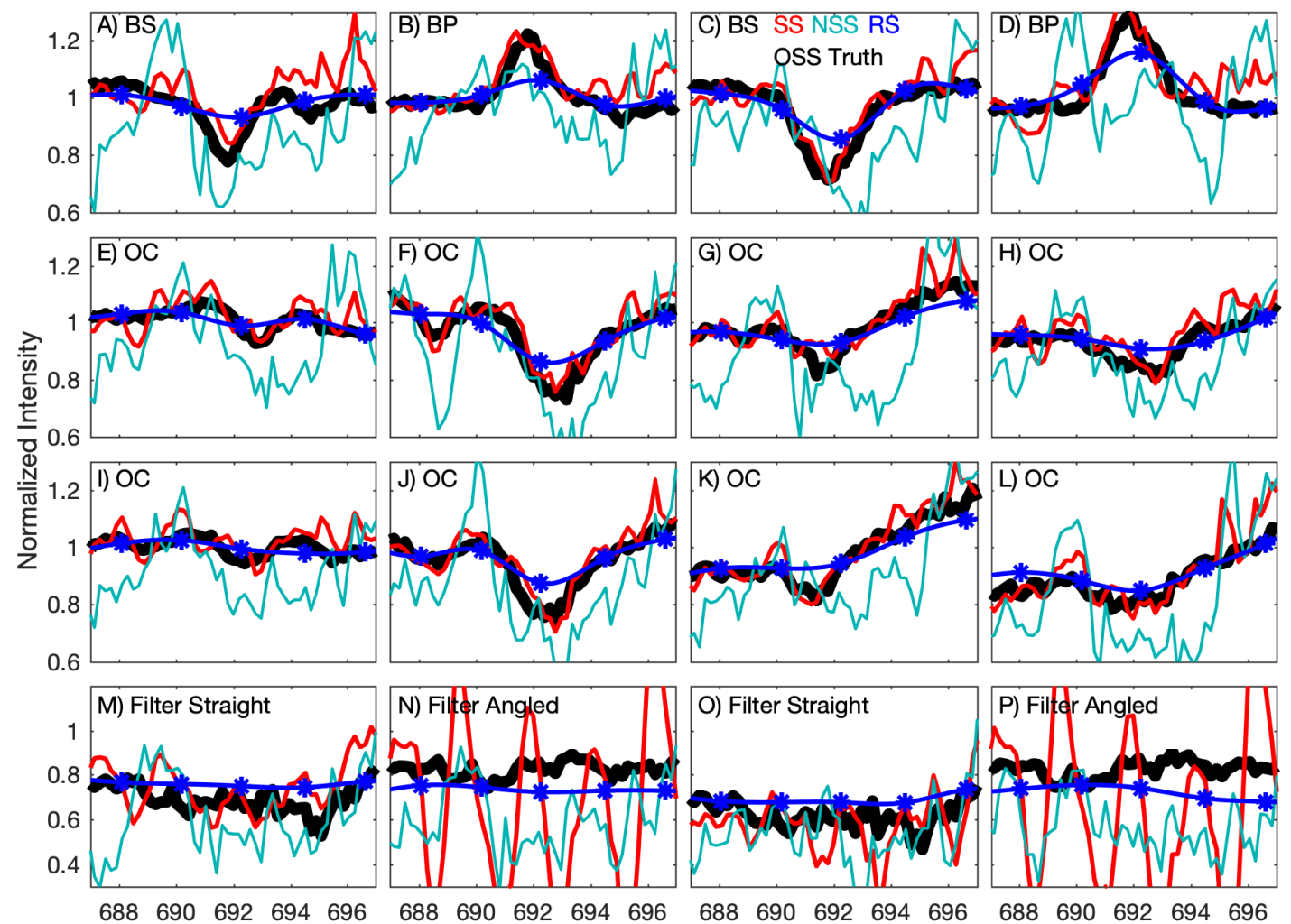

Wavelength $(\mathrm{nm})$

Figure 4-9: Example spectra produced from testing data with a variety of different statistics including laboratory generated band-stop (BS), band-pass (BP), and ocean color (OC) and two short-pass transmission filter's (Filter) straight on (Straight) and at an angle (Angled). Generally, the SS SVRs can capture medium frequency spectral features in these signals at the cost of noise in the higher frequencies. 
The spectral frequency data supports the visual analysis (Fig. 4-10). The single sided amplitude frequency of a single predicted test spectra (Fig. 4-9, F) from the SS SVR (Fig. 4-10, SS, red, top panel) matches the truth data (OOS Truth, black, top panel) just before and after the Nyquist. However, the SS SVRs poorly represent higher frequency components of the 'truth' measurements of the laboratory generated ocean color dataset, overrepresenting medium-high frequency content. The re-scaled camera measurements achieve slightly higher accuracy at the lowest frequencies (Fig. 4-10, SS, blue, bottom panel), while severely under-representing the higher frequencies (Fig. 4-10, RS, blue, top panel). The NSS dataset performs the worst across almost the entire frequency range (teal), overestimating the spectral content across the entire spectral range. All 3 perform poorly after a spectral frequency of about $1 \mathrm{~nm}^{-1}$ (Fig. 4-10, bottom panel). Overall, the SS SVR predicted data provide a benefit on the entire ocean color dataset until about $0.5 \mathrm{~nm}^{-1}$ (Fig. 4-10. SS, red, bottom panel), which is beyond the Nyquist, after which the SS SVRs poorly represent the spectral frequency content in the simulated ocean color datasets.

The SS SVR predicted spectra have the highest correlation (Table 4.1, row 1 labeled 'Original') and lowest median MAPD (Table 4.2, row 1 labeled 'Original') when compared to the truth values measured by the Ocean Optics spectrometer on the training and testing subsets. For the ocean color subset, the SS SVR predicted spectra have slightly poorer correlation and MAPD relative to the RS spectra, likely due to the higher noise in the medium-high frequencies. For the band-pass/band-stop (BP/BS) subset, the correlation is actually higher despite the MAPD being lower. Finally, for the real-world filter subset all three perform poorly in terms of both correlation and MAPD. Overall, on the original, unaltered, dataset the SS SVRs perform best when used on unseen data with the same statistics as the training data, but generally not as well on data with different statistics, seemingly because of the poor representation of the medium-high frequency spectral content.

\subsubsection{Impact of noise on super-resolution: Generalization of noisy SVRs to laboratory generated ocean color, band-pass/band-stop, and real- world signals}

The SS SVRs provide significant benefit to predicting spectra within the testing subset, despite a range of noise sources characteristic of ocean color measurement (Table 4.1 \& 4.2. rows 2-4, columns 4-6). When residual glint (Glint), Gaussian shot-noise (SNR10), or 

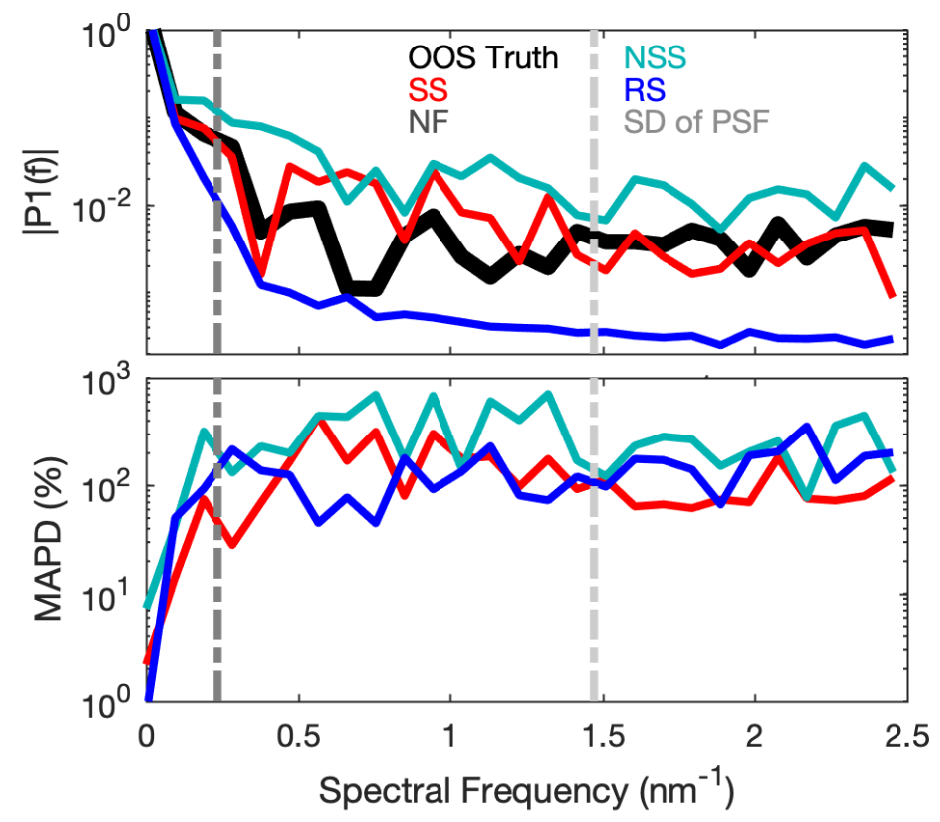

Figure 4-10: The single sided power spectrum of the prediction produced from the SS SVRs (red, top panel) on an example laboratory generated ocean color signal (Fig. 4-9, F) matches the truth spectrum (black) at low frequencies, even slightly past the pixel limited Nyquist frequency (dark grey dot dashed line), but poorly represents higher frequency content, overestimating at medium-high frequencies. The NSS SVRs (teal) match the frequency content until half of the Nyquist frequency, while the re-scaled raw pixel data (blue) only matches the spectral content at the lowest spatial frequencies and underestimates higher spectral frequencies. The median absolute percent difference (MAPD) calculated between the OOS Truth measurements spectral content and the spectral content of the SS, NSS, and RS predictions for all of the generated ocean color signals demonstrates the benefit of the SS SVRs at accurately representing the low spectral frequencies surrounding the Nyquist (bottom panel). 
a combination of glint and Gaussian shot-noise (Glint + SNR10) is added to the signal, the SS SVRs still provide the highest accuracy and correlation of the 3 techniques on the testing data. However, when the SS SVR technique is used to predict laboratory generated ocean color or band-pass/band-stop signals with different statistics than the training data, or used to estimate the spectral transmission efficeincy of a real-world filter, the SS SVRs no longer provide the lowest MAPD (except in one case), though they do provide slightly higher correlation on the $\mathrm{BP} / \mathrm{BS}$ data (Table $4.1 \& 4.2$, rows 2-4, columns 7-12). In summary, the SS SVRs provide high accuracy metrics on the testing subset, which has similar statistics as the training dataset, but not on laboratory generated or real-world data-sets with different statistics.

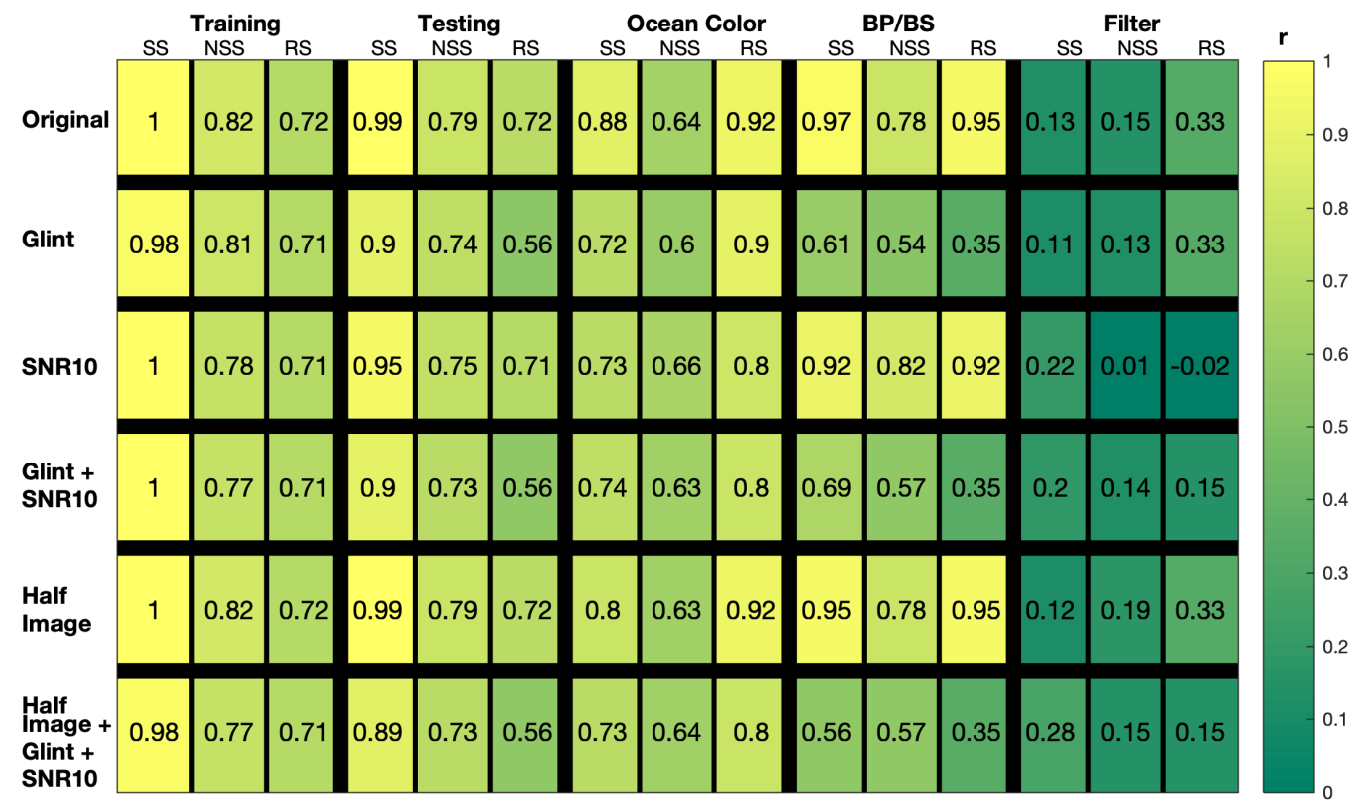

Table 4.1: Pearson's correlation coefficient (r) calculated between the 'truth' values and the predicted spectral data for each subset from the SVRs trained with spectral shift (SS), the SVRs trained without spectral shift (NSS), and re-scaled spline interpolated camera measurements (RS). Clearly the SS SVRs achieve very high correlation with the testing sets for the original dataset, data with simulated residual glint (Glint), data with a reduced signal-to-noise ratio of 10 (SNR10). This benefit even holds when just using the right half of the images (Half Image), even with added residual glint and an SNR of 10 (Half Image + Glint +SNR10). Some of the accuracy benefit holds for the laboratory generated bandpass/band-stop data, but the benefit does not hold for laboratory generated ocean color data or real world filter data. 


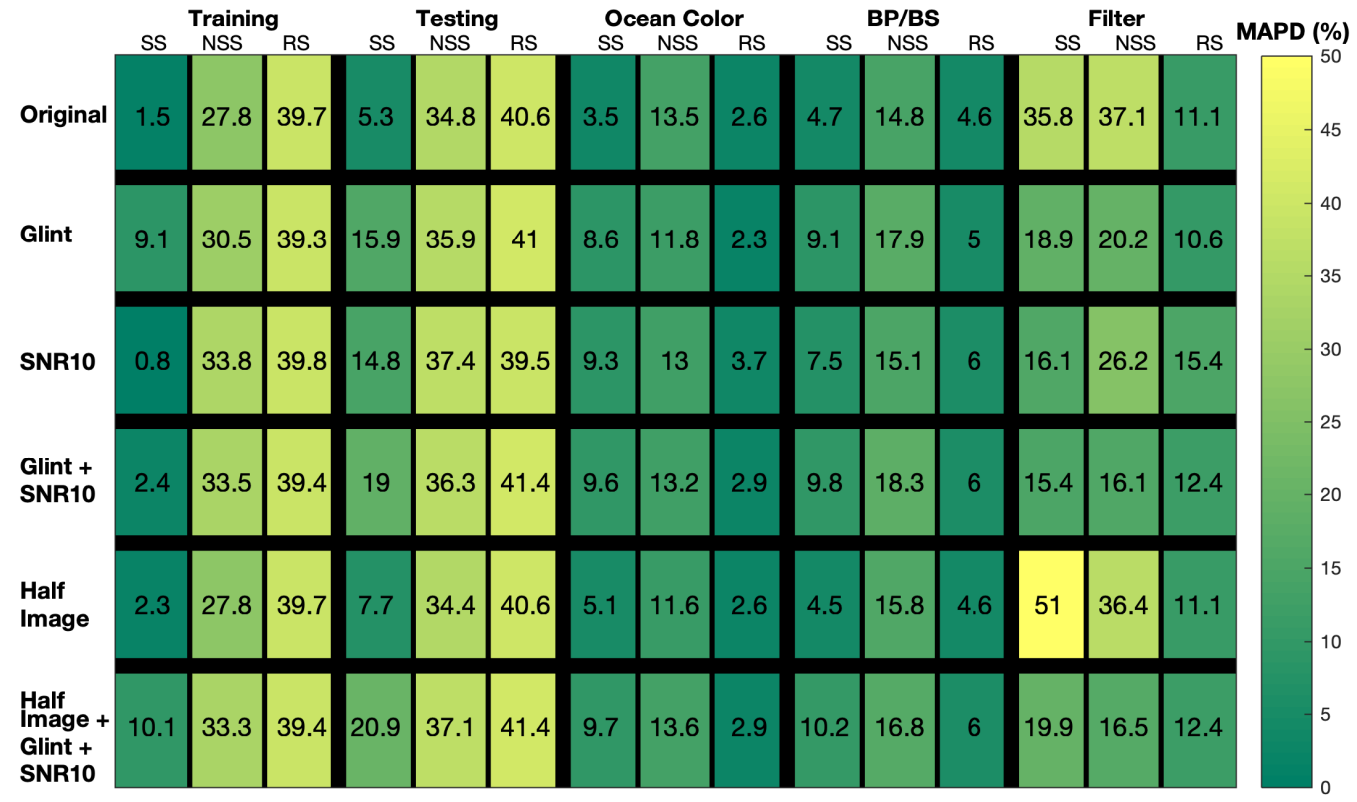

Table 4.2: The median of the MAPD calculated for each of the 53 output spectra from the SVRs trained with spectral shift (SS), the SVRs without spectral shift (NSS), and the re-scaled spline interpolated camera measurements (RS), calculated on the different data subsets (topmost column labels). Clearly the SS SVRs predicted spectra achieved much lower error on the testing dataset, but these accuracy benefits do not hold for the laboratory ocean color, band-pass/band-stop (BP/BS) data, or real-world filter measurements (right three columns). 


\subsubsection{Spatial subsets for spectral super-resolution}

To use the SS SVRs for the example task of increasing the spectral resolution in images of the interface between two water bodies, half of the image would need to be used for each section. When only half of the image is input into the SVRs, the SS SVRs still provide the highest accuracy on the testing dataset, and only loses minimal accuracy while maintaining a very similar correlation relative to using the entire spatial domain (Table $4.1 \& 4.2$, rows 1 and 5, column 4). Unfortunately, the half-image input still fails to generalize to laboratory generated or real-world datasets with different statistics (Table 4.1 \& 4.2, row 5, column 7-15). Interestingly, when noise characteristic to ocean color measurements is applied to the half image, including both simulated glint and high Gaussian shot-noise, the testing error is still highest for the SS SVRs predictions and similar to that when using the full input image width (Table $4.1 \& 4.2$, rows 4 and 6 , column 4 ), however again this set fails to generalize to datasets with different statistics (Table 4.1 \& 4.2, row 6, column 7-15). Overall, using just half the spatial image still provides a significant accuracy increase over the other prediction methods, but this benefit does not persist for subsets with different statistics from the training dataset.

\subsection{Discussion}

To our knowledge, this study is the first use of machine learning to leverage spectral shift in images captured by an imaging spectrometer to increase the spectral resolution of beyond its nominal pixel limited resolution. Leveraging the spectral shift for increased spectral resolution was previously proposed by Watanabe et al. WWatanabe and Furukawa, 2018 and explored in Kitano et al. [Kitano et al., 2020|, but not rigorously tested under a wide range of sources of contamination characteristic of ocean color signals. The results of our study focused on one of the most difficult applications for this technique, data with similar noise

and statistics as real-world ocean color measurements, but the lessons learned from these results could easily be applied to real-world terrestrial remote sensing or industrial line-scan applications which do not suffer from these limiting noise sources. 


\subsubsection{Sub-pixel spectral shift enables spectral super-resolution on datasets with similar statistics}

The higher predictive accuracy of the SS SVRs over the NSS SVRs and RS prediction method in the training and testing subsets in the original dataset (Table 4.2 , row 1 , columns 1-6) is the result of the spectral shift, not an increase in the shot-noise limited SNR or other optical effects. The SNR of just the NSS spatial section over the entire training, validation, and testing subsets is $\sim 23$ (calculated by taking the spatial mean divided by the standard deviation of this subset, and taking the median over all the spectra), and so would only have an error of at most $\sim 5 \%$. While there are other optical effects on the imager, such as keystone, stray light, and vignetting, the additional information required to increase the accuracy needs to come from a sub-pixel shift in the spectral content at each pixel, so theoretically none of these optical effects would provide the required non-redundant information. Finally, if the accuracy gain came strictly from learning dictionary-based correlation between the course spectral resolution measurements from the imaging spectrometer and the high spectral resolution measurements from the Ocean Optics spectrometer, the NSS SVRs would have been able to match the accuracy of the SS SVRs. Therefore, the increase in the accuracy when using the entire spatial domain must come from the spectral shift (Fig. 4-4, and not from another optical effect. In summary, the cause of the increased accuracy at finer spectral resolutions is not the result of increased SNR, other optical aberrations, or just a dictionary-based learning of the underlying dataset, but instead a learned relationship between the spatial pixels and the spectral shift.

\subsubsection{SVRs can be trained to be insensitive to environmental and optical noise typical of sub-meter ocean color images}

Two difficulties in ocean color remote sensing are the low SNRs available in the near-infrared (NIR) and the residual offsets remaining after correcting the ocean color data for glint O'Shea et al. 2020. Both of these offsets can be included during training to force the

SVR to be robust to these types of noise sources. Although noise sources can decrease the effectiveness of spectral super-resolution non-linearly [Sezer and Altunbasak, 2018], the SS SVRs were still able accurately recover testing data at the SNR limit of ocean color data (Table $4.2 \& 4.1$, rows 2-4, columns 4-6). An SNR of 10 was chosen as it is the the minimum 
value typically seen in ocean color measurements captured by imaging spectrometers O'Shea et al. 2020]. By demonstrating that the SS SVRs can be trained to be insensitive to the lowest and highest available SNRs, we demonstrate that this technique could provide benefit across the entire range of typically observed SNRs. While some of the accuracy gains ( $\sim 5 \%)$ could be due to an increased SNR in the full spatial resolution dataset relative to the local region representing data without spectral shift, they cannot explain the nearly $25 \%$ accuracy gain on the testing set relative to the NSS or RS data (Table 4.2, rows 2-4, columns 4-6). The second difficulty with using ocean color data is the non-Gaussian residual noise from imperfect glint correction. While the SS SVRs were retrained with non-Gaussian residual glint using histograms to match the ocean color data, the actual under- and overshooting of the glint correction algorithms may have more spatial correlations than our simulated method [Mobley, 2016], since the under- and over-corrections are often adjacent. The adjacency is due to the shape of capillary waves changing the reflection coefficient on fine spatial scales. In essence, that means that the reported MAPE and correlation coefficient is a lower bound (i.e., worst case scenario) as the simulated glint has reduced information content relative to the real-world glint. Since the SS SVRs reduce the predicted error by nearly a factor of 2, the fact that the real-world measurements of the spatial structure could further increase the accuracy is quite promising. Real-world truth and training measurements will need to be collected to implement this technique on sub-meter ocean color data collected in the field. Overall, the accuracy of the SS SVR predictions was significantly hindered by the combination of Glint and SNR, but the technique still provided an accuracy benefit even at the lowest typical SNRs of ocean color data while experiencing previously unseen residual glint distributions.

\subsubsection{SS SVRs weakly generalize to datasets with different statistics}

The attempt to force generalization of the SVRs by providing training data that was random and uncorrelated in both spectral intensity and location was only partially successful. The SS SVRs better represented the frequency content at and above the Nyquist on ocean color data, but at the cost of increased noise at higher spectral frequencies (Fig. 4-10. Despite the better representation of frequencies surrounding the Nyquist, the general error metrics, including the correlation coefficient and MAPD, performed worse than the simple RS prediction method (Table 4.1 and 4.2, columns 7-12), due to the higher frequency noise. 
However, despite the medium-high frequency noise, the SS SVRs did beat or match the correlation coefficient for predicting band-pass and band-stop spectra using the RS and NSS methods (Table 4.1, columns 10-12). The poor generalization of the SS SVRs to the ocean color dataset and $\mathrm{BP} / \mathrm{BS}$ dataset could be due to a variety of factors including the SS SVRs attempting to learn high frequency components using the statistics of the training dataset, the SS SVRs identifying the spectral locations used when constructing the training spectra, or the shape of the input spectrum being limited by the spectrometers spectral blur function. While the super-resolution techniques did not generalize well to ocean color data, all three techniques poorly recovered the filters transmission spectra (Table 4.1 and 4.2 , columns 10-12). The poor recovery from the camera could be due to differences in the efficiency of the polarization states induced by the filter, which may change when angled relative to the optical axis, which would change the intensity measured by the imaging spectrometer and Ocean Optics spectrometer in different ways. Overall, the SS SVR technique provides better representation of low frequencies, but at the cost of medium-high frequency noise.

A combination of training sets with higher frequency spectral content and an unknown set identification layer could be used to provide additional accuracy and confidence in applying the spectral super-resolution technique to ocean color data. One approach could use a new training dataset with random and uncorrelated spectral features, containing higher spectral frequencies, and a wider variety of statistics. Another training approach could instead attempt to train using data with similar statistics as the target ocean color data, by utilizing simulated high $(<1 \mathrm{~nm})$ spectral resolution ocean color data to train the SS SVRs. Unfortunately, this would require collection of higher spectral resolution ocean color data in a variety of ocean color scenarios to get a broad enough representation, as the only available dataset with sufficient spectral resolution is in a oligotrophic region that exhibits little variety in its ocean color signal. After retraining the SS SVRS on one of these new datasets, an OpenMax layer could be added to the pipeline to identify if previously unseen images fit within the in-training set images [Bendale and Boult, 2015], and therefore the SS SVR would produce accurate spectral super-resolution predictions, or if they fell into an 'unknown' class with different statistics, and would therefore produce inaccurate spectral super-resolution estimates. Through a combination of retraining on broader more representative datasets and filtering the input data, the spectral super-resolution results from this approach could be more widely accepted for ocean color remote sensing. 


\subsubsection{Comparison to alternative spectral super-resolution approaches}

The spectral content is better represented by the SS SVRs than other predictive techniques until atleast 4 times the Nyquist frequency in the testing subset (Fig. 4-8), but only a factor of 2 in the Ocean Color dataset (Fig. 4-10). While not a direct comparison due to the differences in the instruments spectral imaging techniques, the focal plane array shift applied to a Fourier transform spectrometer by Watanabe et al. Watanabe and Furukawa, 2018 provided a spectral increase of nearly a factor of roughly 2.5. Other spectral super-resolution techniques which add optical aberrations to the system can increase spectral resolution by more than a factor of 10 Konishi et al. 2016, but they come at the cost of needing to modify the optical system. Future research should compare our results to those of Kitano et al. Kitano et al., 2020], when their quantitative results become available, as they use the most similar optical setup with different computational approaches. Overall, the SS SVRs provide similar spectral resolution benefits to a grating based imaging spectrometer as they did to a Fourier transform imaging spectrometer on commercially available spectrometers, without needing to modify the optical system.

\subsubsection{Implications for adaptive sensing of the ocean's color}

The investigated SR technique will allow for new adaptive sensing capabilities, once the technique is proven to generalize in real-world ocean color scenarios. Specifically, the altitude of a drone imaging a specific water body could be varied, to provide a homogeneous image of a particular region of interest. In ocean color remote sensing this would translate to reducing the altitude so a target of interest, such as a patch of algae on the surface of the ocean, fills the entire view angle of the imager and provides the full benefit of the spectral SR algorithm. In this way users can cover large areas at the base spectral resolution of the imager, but when they find particular regions of interest they can reduce the altitude to capture at the highest possible spectral resolution by leveraging the spectral shift in the imager. The proposed mapping method allows users to collect both high spatial and spectral resolution data. 


\subsubsection{Limitations of the laboratory based study and future work}

The laboratory-based study is limited by multiple factors which prevent directly applying this technique to real-world data captured by an imaging spectrometer deployed from a drone. First, training images collected by the current optical setup use a fiber optic cable as input to the imaging spectrometer, so the learned relationships may not translate to images collected without the fiber optic cable as input. An alternative optical setup, consisting of a brighter source with an acousto-optic tunable filter going into an integrating sphere, could enable the simultaneous acquisition of high enough intensity signals to train the system without a fiber optic input to the imager. Second, due to the low signal emanating from the monochromator, the camera system must be trained with a high gain which is typically not used in real world measurement, as it can lead to higher noise from the amplifier. The previously mentioned optical setup could potentially eliminate the need to use such a high gain. Third, the exact spectral shift learned in the lab could be sensitive to changes in the FPA due to temperature, and mechanical misalignment [D'Odorico et al., 2010], which could occur during flight of a drone and decrease the benefit of the technique. Fourth, the technique may provide lower accuracy gains when binning the pixels in the spatial dimensions or further binning the spectral dimension. Binning in the spatial dimension would reduce the measured changes in the spectral shift, and inherently limit the achievable accuracy. Binning the pixels in the spectral dimension would reduce the relative size of the spectral shift (relative to the spectral signal covered by an individual pixel). Future research should focus on training the system without fiber optic input to the system, testing the technique from a drone at altitude, and determining how binning in the spatial and spectral dimensions limits the accuracy benefit.

\subsection{Conclusion}

The sub-pixel spectral shift contained within an example commercially available imaging spectrometer provided sufficient non-redundant information for SVRs to better represent spectral frequencies up to four times the Nyquist, in datasets with similar statistics as the training dataset. While the same SVRs increased the visibility of spectral features in laboratory generated ocean color signals specifically selected for their spectral variability, and increased the accuracy of frequency representation beyond the Nyquist, they added signifi- 
cant noise at higher frequencies that decreased the overall accuracy. The SVRs were tolerant of high noise and simulated non-Gaussian residual glint characteristic of sub-meter ocean color measurements, however again they were unable to generalize to datasets with different statistics. Finally, SVRs leveraging spectral shift continued to provide high accuracy when only half of spatial locations in each image were used, even with high noise sources. Overall, the results are promising for increasing the accuracy on datasets with similar statistics as the training dataset, but more research is required to create training datasets that force the spectral super-resolution technique to generalize to datasets with similar statistics as the target ocean color data. 


\section{Chapter 5}

\section{Conclusion}

\subsection{Dissertation's Contributions to Sub-meter Ocean Color Remote Sensing}

This dissertation redesigned the three steps within the satellite ocean color remote sensing pipeline which benefited the most from adaptation to sub-meter scales. The specific steps included the removal of surface-reflected light despite capillary wave induced fluctuations, the design of the optics and electronics considering the increased opto-electronic noise, and the post-processing of over-sampled ocean color images in spite of environmental and optical noise. When the individual steps are combined, to form the sub-meter ocean color remote sensing paradigm, they build upon one another to enable a range of more advanced sub-meter ocean color research opportunities in remote heterogeneous regions. First, the individual contributions are summarized below, then an essential sub-meter ocean color application enabled by the combination of the chapters is presented, and finally future work to further advance the sub-meter paradigm is proposed.

\subsubsection{Chapter 2 Contributions}

Of the three traditional glint correction approaches tested in Chapter 2, the Lee et al. Lee et al. 2010 optimization method worked best for sub-meter ocean color optical constituent retrieval, despite fluctuations in the surface reflectance induced by capillary waves. This chapter also identified that a polarization filter achieved similar accuracy as the optimization method for chlorophyll concentration estimation, which is useful for multispectral imagers with limited spectral content. The long-term deployment demonstrates that the glint 
correction processes function in a wide range of environmental conditions. These spectral glint correction techniques provide an alternative to the spatial filtering approaches typically applied to sub-meter ocean color data. Since the spectral corrections maintain the full spatial resolution, they can leverage information contained within these images for further post-processing (e.g., the spectral super-resolution technique investigated in Chapter 4)

\subsubsection{Chapter 3 Contributions}

The simulation framework presented in Chapter 3 provides users with the ability to identify potential opto-electronic induced engineering constraints, and solutions, prior to deployment. The framework identified that the opto-electronic noise and limited dynamic range of the available imager would limit the accuracy of mapping algal biomass simultaneously from the ocean and sea ice in the marginal ice zone. With this knowledge, researchers determined a potential solution, using the lower efficiency second order measurements for mapping algae within the brighter sea ice, prior to deployment to this remote region. Per the identified hardware limitations and solutions of this example, the framework is particularly useful in oceanographic settings where the instruments cannot be tested ahead of time (i.e., in regions where month long cruises may be the only means of assessing instruments in certain regions).

\subsubsection{Chapter 4 Contributions}

The machine learning based spectral super-resolution (SR) technique presented in Chapter 4 leveraged sub-pixel spectral shift within images of homogeneous targets to increase the predictive accuracy of spectral frequencies beyond the Nyquist. This SR technique serves as an alternative to binning for spatially homogeneous regions, providing increased spectral resolution instead of increased SNR. Additionally, the SR technique was trained to be insensitive to additive Gaussian and non-Gaussian noise sources characteristic of sub-meter ocean color remote sensing on images with similar statistics as the training dataset. The benefits of this SR technique also persist when using subsets of the spatial domain, such as the right half of the image, which is useful for specific ocean color applications (e.g., imaging interfaces). While this chapter focused on noise sources and image subsets characteristic of one of the hardest applications, ocean color remote sensing, this technique is broadly applicable to a wide range of other industrial and agricultural remote sensing tasks. 


\subsubsection{A New Paradigm for Sub-Meter Ocean Color Mapping in Spatially Heterogeneous Regions}

By combining the results of these chapters into the sub-meter ocean color remote sensing paradigm, researchers can fully leverage information contained within homogeneous subsections of remote heterogeneous regions (Fig. 5-1). The accuracy benefits of each step within the sub-meter ocean color paradigm are enhanced by the successful implementation of previous steps. The basic framework for applying these chapters to a unique ocean color sensing scenario starts with seeding the simulation framework using satellite, aircraft, or simulated measurements from the region. With the results from the simulation framework, a researcher can then optimize the optics, detection algorithms, and engineering design, which is necessary to collect high quality data. The selected camera system can then be deployed to the region with the designated operational parameters and view-angle, where it will collect high fidelity data that isn't limited by saturation or high opto-electronic noise. The high fidelity remote sensing measurements collected from the deployment can then more accurately estimate and remove glint, as the high fidelity remote sensing measurements will better match the simulated ocean color values in the optimization-based glint correction approach and more accurately represent the NIR signal. Finally, by reducing glint induced heterogeneity, the spectral SR technique can provide more accurate results. Clearly the successful implementation of each step in the sub-meter paradigm benefits successive steps by providing higher fidelity data.

Many potential sub-meter ocean color research opportunities are enabled by the combination of these research chapters, but not every opportunity requires each chapter (Table 5.1. Chapter 2, which focused on glint removal at sub-meter scales, is broadly applicable to all ocean color applications, ranging from measuring sediment plumes from dredging using drones to mapping glacial outflow from an Aerostat. However, the contents of this chapter are not useful for measuring elements on the surface of the ocean, such as surface algal blooms or algae within sea ice, as they do not suffer from glint. Chapter 3, which dealt with simulating the impacts of opto-electronic noise on specific dynamic regions, is particularly useful for applications with unique ocean color signals in difficult to reach locations. Examples include measuring phytoplankton community structure and concentration in the marginal ice zone or measuring the CDOM composition at an Arctic river delta (Table 5.1. 


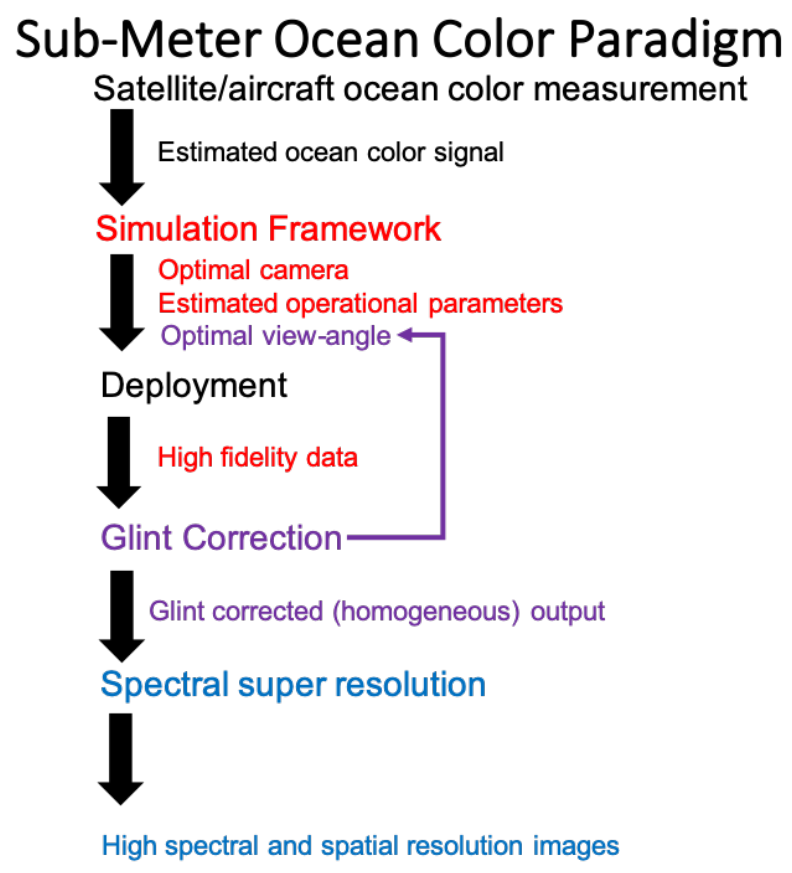

Figure 5-1: The individual chapters combine to increase the fidelity of the measurements at successive steps. To apply this framework to unique ocean color scenarios, one starts with a satellite estimate, passes it through the simulation framework to identify potential engineering constraints. The researcher then deploys the system with the pre-selected parameters, applies the selected glint algorithm to reduce heterogeneity, and finally applies the spectral $\mathrm{SR}$ technique to increase the spectral resolution in homogeneous regions within each image. 
rows 6 and 7). This chapter is less essential for local or well characterized regions (e.g., Table 5.1. row 5, mapping sediment plumes from dredging), as the instruments can easily be tested in these regions. Chapter 4, which enables the trade between spatial and spectral resolution during post-processing, will be beneficial for regions that require high spatial resolution for separation of distinct regions that are spatially homogeneous, but require high spectral resolution (e.g., Table 5.1. rows 6 , measuring phytoplankton composition and concentration in the marginal ice zone). Even though each chapter is not necessary for each application, the combination of the chapters enable a wide range of potential sub-meter ocean color applications from different platforms in remote regions.

The full benefits of the sub-meter paradigm are most obvious from a scientifically important example application, such as mapping algal community structure and biomass within the sea ice and ocean during spring melts in the marginal ice zone. The first step of mapping algal biomass was already presented in Chapter 3, where Aircraft images and Hydrolight generated data were used to seed the simulation framework and determine the limitations induced by the wide dynamic range when simultaneously imaging oceanic and sea ice algae in this remote region. The simulation framework identified the wide dynamic range limitation, and researchers developed the potential engineering solution of using the second order to simultaneously to collect high fidelity remote sensing measurements from the ocean and sea ice. With the view-angle set by the selected glint correction process, researchers could proceed with deployment and collect remote sensing data without saturating the sea ice signal or limiting opto-electronic noise in the oceanic signal. The researchers would then apply glint correction approaches to remove surface reflected sky light, reducing heterogeneity imposed by changes in the Fresnel reflectance induced by surface waves in the oceanic signal. Finally, after collecting high SNR unsaturated data by using the simulation framework and then glint correcting that data, the images with reduced glint fluctuations can be post-processed to trade the spatially over-sampled ocean color within cracks in the sea ice for spectral resolution. The additional spectral resolution provides additional information for the ill-posed problem of phytoplankton functional type identification. Overall, it is clear that this paradigm is useful for collecting high quality data in remote and unique ocean color scenarios. In fact, applying this paradigm to a single real-world contribution would merit multiple years of research, adapting and refining the individual steps within the sub-meter ocean color remote sensing paradigm, to refine the full process for real-world applications. 


\begin{tabular}{|c|c|c|c|c|}
\hline Applications & \multirow{2}{*}{$\begin{array}{l}\text { Scientific and/or societal } \\
\text { justification }\end{array}$} & \multirow{2}{*}{$\begin{array}{l}\text { Chapter } 2 \\
\text { (Glint } \\
\text { Correction) }\end{array}$} & \multirow{2}{*}{$\begin{array}{l}\text { Chapter } 3 \\
\text { (Simulation } \\
\text { Framework) }\end{array}$} & \multirow{2}{*}{$\begin{array}{c}\text { Chapter } 4 \\
\text { (Spectral SR) }\end{array}$} \\
\hline Drone & & & & \\
\hline Coastal ocean color after storm events & $\begin{array}{l}\text { Identify runoff risk, understand } \\
\text { immediate impact on algae }\end{array}$ & $\checkmark$ & $\checkmark$ & \\
\hline Algal bloom development in reservoirs & Early detection and treatment & & & $\checkmark$ \\
\hline Sediment plumes from dredging & Regulation of turbidity & $\checkmark$ & & \\
\hline $\begin{array}{l}\text { Phytoplankton community structure } \\
\text { and concentration, in sea-ice and } \\
\text { ocean, in MIZ }\end{array}$ & $\begin{array}{l}\text { Understand seasonal biomass } \\
\text { distribution \& changes due to } \\
\text { warming }\end{array}$ & $\checkmark$ & $\checkmark$ & $\checkmark$ \\
\hline CDOM composition along Arctic river & $\begin{array}{l}\text { Understand spatial dependence of } \\
\text { hyperspectral CDOM signature }\end{array}$ & $\checkmark$ & $\checkmark$ & $\checkmark$ \\
\hline $\begin{array}{l}\text { Subpixel variations in OACs impact in } \\
\text { satellite retrieved estimates }\end{array}$ & $\begin{array}{l}\text { Approximate uncertainty of global } \\
\text { and regional satellite estimates }\end{array}$ & $\checkmark$ & & \\
\hline Mapping cruise ship effluent discharge & Inform regulation of cruise ships & $\checkmark$ & & \\
\hline Impact of wildfires on OACs & Water quality advisories & $\checkmark$ & & \\
\hline Desert springs & $\begin{array}{l}\text { Replace in-situ monitoring of } \\
\text { remote water sources }\end{array}$ & $\checkmark$ & & \\
\hline $\begin{array}{l}\text { Mapping of green/red tides (i.e., } \\
\text { Sargassum blooms) }\end{array}$ & $\begin{array}{l}\text { Water quality advisories, early } \\
\text { detection, understand formation }\end{array}$ & & & \\
\hline \multicolumn{5}{|l|}{ Aerostat } \\
\hline $\begin{array}{l}\text { Glacial outflow during melting events } \\
\text { impact on OACs }\end{array}$ & $\begin{array}{l}\text { Impact of glacial melt on regional } \\
\text { ocean health }\end{array}$ & $\checkmark$ & $\checkmark$ & $\checkmark$ \\
\hline \multicolumn{5}{|l|}{ Stationary Tower } \\
\hline $\begin{array}{l}\text { Autonomously image arctic freshet to } \\
\text { retrieve CDOM signature }\end{array}$ & $\begin{array}{l}\text { Determine each watershed's } \\
\text { hyperspectral contribution }\end{array}$ & $\checkmark$ & $\checkmark$ & $\checkmark$ \\
\hline $\begin{array}{l}\text { Sediment concentration during } \\
\text { hurricane events }\end{array}$ & $\begin{array}{l}\text { Measure particle concentration } \\
\text { for use in paleo hurricane } \\
\text { reconstruction }\end{array}$ & $\checkmark$ & & \\
\hline CDOM measurement at river outflow & $\begin{array}{l}\text { Inform logging regulation, identify } \\
\text { illegal logging }\end{array}$ & $\checkmark$ & $\checkmark$ & $\checkmark$ \\
\hline $\begin{array}{l}\text { Monitoring of oyster on local water } \\
\text { quality }\end{array}$ & $\begin{array}{l}\text { Achieve higher yields, inform } \\
\text { aquaculture location }\end{array}$ & $\checkmark$ & & $\checkmark$ \\
\hline Microplastic mapping across river & $\begin{array}{l}\text { Identify and regulate local } \\
\text { microplastic contributions }\end{array}$ & $\checkmark$ & & \\
\hline
\end{tabular}

Table 5.1: Example sub-meter ocean color remote sensing applications (first column) that leverage a range of deployment platforms to meet specific scientific and societal needs (second column). The chapters of the dissertation (right three columns) that benefit each application are denoted with check marks. 


\subsection{Future Work in Sub-Meter Ocean Color Remote Sensing}

The contents of this dissertation introduced the preliminary sub-meter ocean color paradigm, but each step within the sub-meter paradigm could be further advanced to provide higher fidelity data.

A critical metric required by researchers to choose between spatial resolution and spectral glint correction approaches is the relative accuracy of both for estimating individual optical constituents (e.g., chlorophyll concentration). Preliminary results suggest that the glint correction techniques provide much higher accuracy than two standard spatial filtering techniques modified for hyperspectral line-scan data from an imaging spectrometer (Fig. 5-2) [Totsuka et al., 2019, Kwon et al., 2020]. In this preliminary comparison with spatial filtering techniques, the first technique (Median Filtered) applied a moving median filter on the uncorrected spatial data to remove fine-scale fluctuations. The non-local mean filter applied in Totsuka et al. Totsuka et al., 2019] was not applied, as the non-local mean filter relies upon the self-similarity within single-band 2D spatial images [Zhuang and Bioucas-Dias, 2018, which is not present in the 1D spatial images produced by imaging spectrometers. Additionally, standard regressions were used instead of fuzzy regressions, to simplify comparison with the other glint correction approaches. The second approach uses Savitzky-Golay filtering on the input $R_{\mathrm{rs}}$ spectrum, with a frame-length equal to 1 degree $(17$ pixels $\sim 35 \mathrm{~cm})$ and an order of 3. The output concentration map is also Wiener filtered with the same frame-length [Kwon et al., 2020]. While the spatial correction methods increase the accuracy beyond the uncorrected data, the spectral-glint correction techniques provide higher accuracy across the view-angle range, without requiring sacrificing spatial resolution. The poor benefit of the spatial algorithms relative to the spectral algorithms is likely due to the fact that the spatial filtering algorithms are not removing the surface reflected light, just fluctuations in the surface reflected light induced by capillary waves on fine scales.

Two more involved future research directions for Chapter 2 could further reduce the impact of capillary wave induced surface-reflected light fluctuations in spatially over-sampled regions. First, the spectral glint correction techniques could be applied to the spatially filtered datasets to reduce the prediction error to beneath the accuracy achieved by just the spectral glint correction techniques. The spatial filtering would provide a better average estimate of the light in these regions. A second glint removal technique that could be explored 


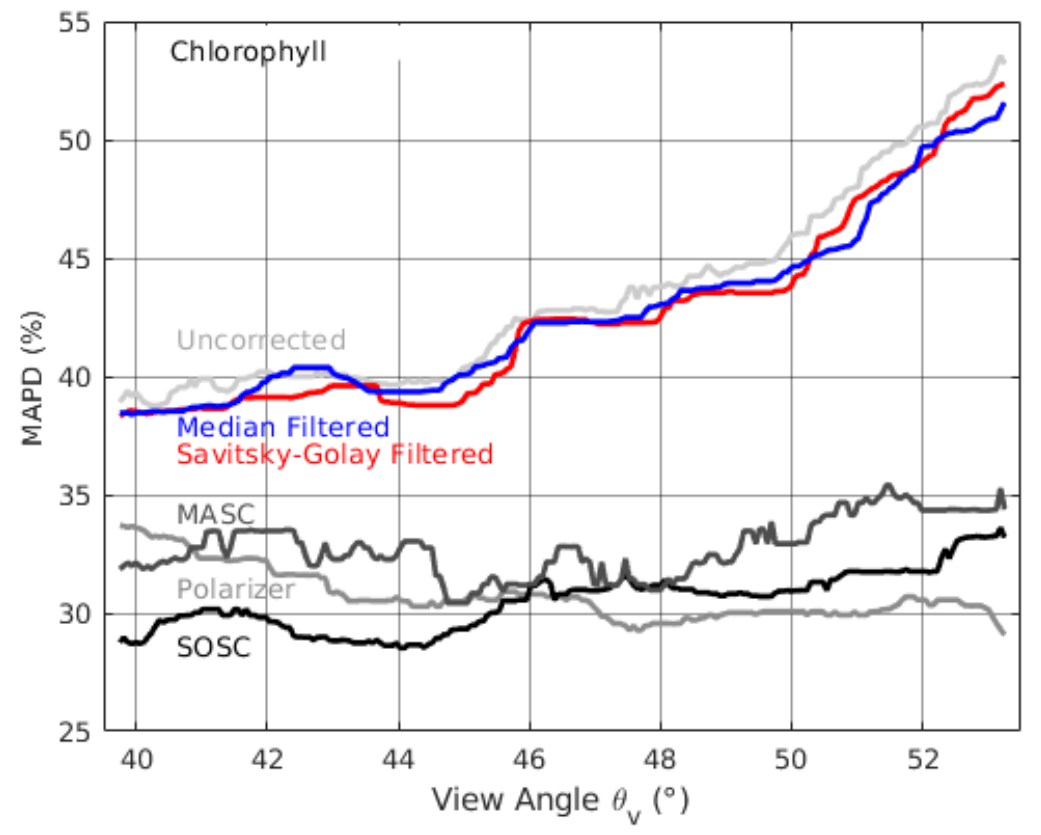

Figure 5-2: The spatial filtering techniques provide a slight reduction in the median absolute percent difference in the estimated chlorophyll concentrations relative to the uncorrected dataset, but sacrifice significant spatial resolution (from $\sim 2 \mathrm{~cm}$ to $\sim 35 \mathrm{~cm}$ resolution) to do so. The spatial correction techniques perform worse than all of the spectral glint correction techniques across the entire view angle range. The results show slightly different values than previously presented view-angle dependent results (Fig. 2-10), as MAPD is used instead of MAPE, and the error estimate was performed on a slightly different testing dataset.

in over-sampled spatially homogeneous regions could focus on leveraging the spatial shape of capillary waves to better estimate the Fresnel reflectance and surface-reflected light Gilerson et al. 2018]. The better estimate of the Fresnel reflectance could seed the optimization-based glint correction process and potentially reduce errors in the residual glint from the spectral glint correction processes [O'Shea et al. 2020]. In summary, future work could leverage the information contained within spatially over-sampled ocean color images to increase the accuracy of spectral glint correction approaches.

The first advancement for the simulation framework from Chapter 3 must be a demonstration and refinement of the framework for a real-world application. Specifically, the simulation framework's results for a specific scenario should be directly compared to results captured by an imaging spectrometer with sub-optimal operational parameters (e.g., fullwell depth or ADC resolution). An initial test could be done using data captured from the the deployment covered in O'Shea et al. O'Shea et al. 2020 and re-sampling the data by 
reducing the ADC's bit-depth or increasing the shot-noise in post-processing. However, the results would be more convincing when applied to a remote real-world dataset with optoelectronic limitations that are not simulated, and with associated in-situ radiometric and WQP estimates. With in-situ radiometric measurements the error's resulting from glint, the estimation algorithm, and the opto-electronics noise can be separated in the real-world dataset, and compared to the simulation results individually. After refining the framework for real-world applications, further advancements should be made for the next generation of spectral imaging technologies. The simulation framework currently only provides the ability to simulate a grating based imaging spectrometer, but snap-shot hyperspectral imagers are the future of sub-meter drone-based ocean color remote sensing, as they provide 2D spatial information without requiring platform movement. Snap-shot hyperspectral imagers are already being tested for ocean color imaging on fine scales Carrizo et al., 2018; Gilerson et al. 2018], but they may suffer from different opto-electronic induced limitations in unique ocean color scenarios. Therefore, the ability to simulate snap-shot hyperspectral cameras is necessary for imaging in remote heterogeneous oceanic regions. Overall, by advancing the real-world requirements and future needs of the simulation framework, it will stay relevant for the next generation of drone-based ocean color imagers.

Chapter 4 introduced a spectral super-resolution technique which requires advancement in specific areas before it can be applied on real-world ocean color data. Obviously the first step to applying this technique to ocean color remote sensing data is to force the technique to better generalize to unseen ocean color datasets. Better predictive results for unseen ocean color datasets could be accomplished by training the algorithm on data with similar statistics as the target ocean color data. Unfortunately, only a single ocean color dataset collected by an instrument in a typically oligotrophic region (the Marine Optical Buoy in Hawaii [Clark et al. 2003 ) provides sufficient spectral resolution for training. Therefore, a new dataset with sub-nm spectral resolution covering a wide range of ocean color scenarios is required to train the SVRs to match the variety of ocean color statistics expected in the global oceans. Another problem with application of this technique to real-world ocean color data is that it will be difficult to determine where mixing in heterogeneous environments ends. Therefore, an important research step for this chapter would be to identify regions within heterogeneous images that were spectrally similar, using algorithms specifically designed for hyperspectral similarity [Wang et al. 2015], so that only those pixels would be used in the 
spectral super-resolution algorithm. It is likely that the selected homogeneous regions will have mild residual heterogeneity, so a final addition to this chapter would be to train the machine learning techniques to be insensitive to mild heterogeneity, as it is unlikely that waters will be perfectly mixed on these scales. This would be best accomplished by a field study, similar to Chapter 2 but with in-situ radiometric measurements, where heterogeneity was included in the real-world measurements. Overall, by refining these specific steps the spectral super-resolution algorithm would be applicable to real-world ocean color data from heterogeneous regions.

\subsection{Applications of Dissertation Research Beyond Ocean Color Remote Sensing}

The new sub-meter imaging paradigm for water bodies enables a wide range of novel applications outside of standard ocean color remote sensing tasks. Example non-traditional tasks that would benefit from the chapters include water quality monitoring of remote desert springs to replace in-situ monitoring, regulation of cruise ship effluent discharge in areas exhibiting weak tidal exchange [Meams et al., 2003], and monitoring microplastics across river outflow to identify their source (Table 5.1). In each case, glint correction is critical for accurately recovering the radiance. The simulation framework is essential for selecting the optimal commercially available imaging spectrometer for detecting microplastics, as the spectral signature of microplastics often occurs in the NIR Garaba and Dierssen, 2020, where the SNR of the commercially available imaging spectrometers are the lowest O'Shea et al. 2020. The increased spectral resolution from the spectral SR technique could, with additional training, be used to provide additional information for identifying algal species within desert springs, which can vary substantially [Shaaban et al., 2015]. Overall, the sub-meter ocean color remote sensing paradigm generates higher quality remote sensing data that is useful for all aquatic applications leveraging imaging spectrometers, not just standard ocean color remote sensing tasks.

The glint correction and simulation framework of Chapters 2 and 3 were necessarily focused on the narrow field of ocean color remote sensing, however the spectral superresolution technique developed in Chapter 4 would also be beneficial within industrial and agricultural applications. One specific example application would be to use these techniques 
on coarse resolution imaging spectrometers for sorting polymers using their NIR and shortwave infrared (SWIR) spectra Moroni et al., 2015; Huth-Fehre et al., 1998; Mauruschat et al., 2016. The technique could be used to reduce the spectral resolution required, and therefore the cost of the imaging spectrometer, by spatially over-sampling the targets on the conveyor belt. Similarly, this technique could be applied for agricultural sorting of bruised apples, which require between 9 and $18 \mathrm{~nm}$ spectral resolution [Lu, 2003], to reduce spectral resolution (and therefore cost) of the imaging spectrometer. While only two specific examples are presented, the potential applications are wide ranging. 


\section{Appendix A}

\section{Determining the analog-to-digital conversion factor}

The spectral signal-to-noise ratio can be calculated theoretically from the number of electrons captured on an imager's focal plane array. The analog-to-digital conversion factor $(g)$ converts the imager-measured digital numbers to electrons. Eq. A1, which holds after correcting for camera noise and pixel-to-pixel variability [Newberry; Janesick, 2007], calculates the analog to digital conversion factor $(g)$ from images of a flat field.

$$
N_{\text {imager,C }}^{2}=(1 / g) S_{C}
$$

Therefore, by calculating the mean $(S)$ and standard deviation $(N)$ of the imager measured counts from a flat field image, it is possible to compute the conversion factor $(g)$.

A correction procedure for bias follows that of Newberry [Newberry]. First, two images are taken of a flat field target at identical exposure times. Each spectral row of the first image is then mean-scaled to match the spectral row mean of the second image, to account for illumination fluctuations between the two images. The first image is then subtracted from the second. From the difference is calculated the standard deviation of the spatial data and the square of that value. The square of the standard deviation of the difference image is twice the single image variance. When the single image variance is plotted against the mean value for each spectra, the inverse of the slope of the line of best fit is equivalent to analog-to-digital conversion factor $(g)$ [Meola et al., 2011; Janesick, 2007]. This conversion factor can then be used to convert the digital number to the number of electrons, so that the theoretical SNR can be calculated.

From this approach a conversion factor of 7.8942 electrons per digital number was ob- 
tained. This value is nearly identical to the conversion factor which covers the full dynamic range of the full well ( $\sim 32,700$ electrons) using the imager's maximum radiometric resolution of 4096 bits (7.9365 electrons per digital number). Given the agreement between these two estimates, the conversion factor of 7.8942 electrons per digital number was used in the theoretical signal-to-noise ratio calculations within this paper. 


\section{Appendix B}

\section{Comparing theoretically and spatially derived SNRs}

A theoretical derivation for SNR was described previously in Introduction subsection 2.1.3. A separate metric of the SNR can also be estimated using a second method based on spatial statistics, referred to hereafter as the spatially-derived SNR. The signal is the mean of the intensity over a range of spatial pixels, and the noise is the standard deviation calculated from the same range [Eq. B1].

$$
N_{j \text { spatially-derived }}=\sqrt{\frac{\sum_{i=j-24}^{j+25}\left(x_{i}-\bar{x}\right)^{2}}{50}}
$$

Here, $N_{j}$ spatially-derived is the spatially-derived noise at the $\mathrm{j}$-th pixel (calculated from the 50 surrounding pixels), $\mathrm{x}$ is the signal at the i-th pixel, and $\overline{\mathrm{x}}$ is the mean signal of the 50 surrounding pixels. When assuming a homogeneous signal without environmental factors, this estimate of SNR derived from spatial statistics is identical to that derived theoretically.

To explore the limitations of the single image radiometric correction we compared the spatial SNR of the radiometrically-corrected imaging spectrometer images to their theoretical shot noise limited precision. One limitation of the radiometric correction approach we used here, which is performed post-deployment, is that the corrected images rely on only a single radiometric image of an integrating sphere. This approach does in fact correct for a significant amount of pixel-to-pixel variability but it is still subject to shot noise errors that may be present in the single image of the integrating sphere (Fig. B-1, Spa. 1). Consequently the spatial SNR from a single image correction (Spa. 1) does not approach shot noise limited accuracy of the original image (Theo.). To approach the shot noise limit a 
larger number of correction images are needed in order to remove the random fluctuations induced by shot noise (Fig. B-1, Spa. 100). The radiometrically-corrected single image SNR curve can be approximated by solving for the variance and mean of the single-image corrected test image (CT) [Eqs. B2 and B3]

$$
\sigma_{\mathrm{CT}}^{2}=E\left[(1 / C)^{2}\right] * E\left[T^{2}\right]-E[(1 / C)]^{2} * E[T]^{2}
$$

where $C$ is a pixel from the correction image, $T$ is the corresponding pixel from the test image, $\sigma_{\mathrm{CT}}{ }^{2}$ is the variance of the single image corrected test pixel, and $E$ is the expected value. We assume that $C$ and $T$ are shot noise limited so that in turn we can assume they exhibit a Poisson distribution.

$$
S N R_{\text {Theo. } \mathrm{CT} 1}=E[T] /\left(E[C] *\left(\sigma_{\mathrm{ct}}\right)\right)
$$

Here, $\mathrm{SNR}_{\text {Theo. CT1 }}$ denotes the estimated theoretical SNR limit due to the single image correction process. Within this paper, the SNR was calculated in the same manner as $\mathrm{SNR}_{\text {Theo. CT1 }}$, the radiometric correction limited value. The difference between theoretical SNR limit and the $\mathrm{SNR}_{\text {Theo. CT1 }}$ limit induced by photon shot noise in the correction image may not be wholly negligible and will depend on the level of environmental noise in the actual observational measurements. 


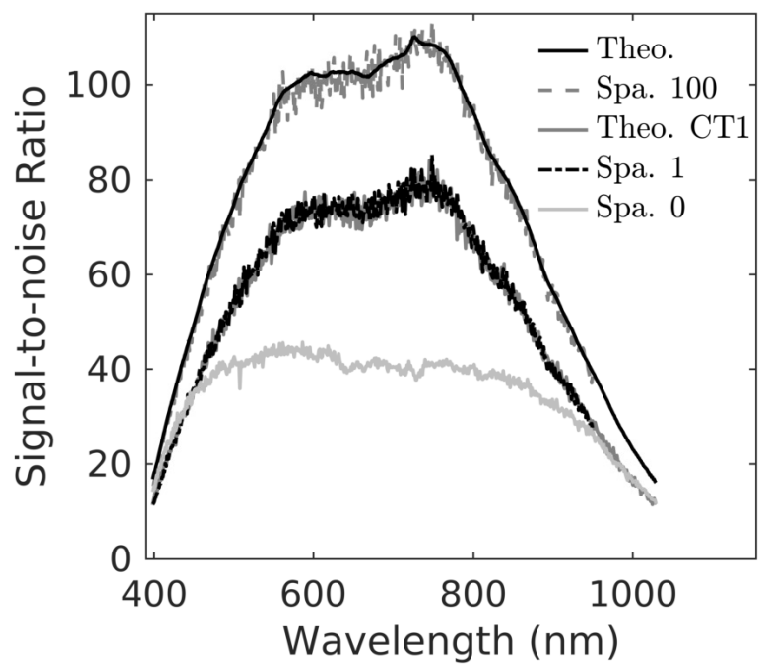

Figure B-1: When no radiometric correction technique is applied (Spa. 0), the pixel-to-pixel variations limit the spatial SNR below the theoretical limit (Theo.). When we radiometrically correct using 100 images, the spatially-derived SNR (Spa. 100) and the theoretically derived SNR (Theo.) match. Only one radiometric correction image is used to correct our observational data set. The spatially-derived SNR using 1 correction image (Spa. 1) can be approximated by the theoretical limit due to a single correction image (Theo. CT1) [Eqs. (B2) and (B3)]. 


\section{Appendix C}

\section{Spectral Optimization Software Correction: modifications}

The original Lee et al. Lee et al. 2010 glint correction approach used an optimization algorithm with only lower bounds that sometimes converged to physically unrealizable remote sensing reflectances. When used with our data set this implementation of the optimization algorithm led to a $>100 \%$ increase in the remote sensing reflectance at $550 \mathrm{~nm}$ for $48.7 \%$ of our hyperspectral imager data [Lee et al., 2010]. We explored the use of a more robust bounded optimization algorithm that would ideally retain a greater amount of our observational data when applying the Lee et al. Lee et al., 2010 SOSC approach. When applying a lower and upper bounded optimization algorithm with a realistic initialization point, we obtained convergence of the SOSC to physically realistic radiances in $99.84 \%$ of our observations. We constrained the algorithm's offset value so that it could not increase the $R_{\mathrm{rs}}$ beyond the measured value, and so that it could not force red wavelengths at $650 \mathrm{~nm}$ negative. We empirically derived the lower and upper bounds for the SOSC parameters P, $\mathrm{G}$, and $\mathrm{X}$ to be [0.003 1], [0.001 1], and [0.0001 0.5] respectively. These values were derived by running the optimization algorithm on individual pixels from a variety of images in the time series and identifying $\mathrm{P}, \mathrm{G}$, and $\mathrm{X}$ values that corresponded with the optimizer not converging to the measured $R_{\mathrm{rs}}$. This empirical derivation of the lower and upper bounds could be done on any remote sensing reflectance dataset to reduce errors in the optimizer.

In addition to unrealistic $\mathrm{P}, \mathrm{G}, \mathrm{X}$, and offset values, we also observed issues with negative estimates of $R_{r s}$ that suggested undefined initialization points. To remedy this problem, for negative first guesses of remote sensing reflectance spectrum at $440 \mathrm{~nm}, 555 \mathrm{~nm}$, or $550 \mathrm{~nm}$ 
we forced the initialization values using the total $R_{r s}$ less the Fresnel reflectance multiplied by the sky remote sensing reflectance. The initial guess $R_{r s}$ was the total remote sensing reflectance, less the Fresnel reflectance multiplied by the sky remote sensing reflectance, less the mean of that signal from $750-800 \mathrm{~nm}$. If those values were still negative, the total remote sensing reflectance was used to set the initialization values. After running the bounded optimization with real initialization points using two different algorithms, interior point and active set, the solution with the lowest error was taken as the final SOSC remote sensing reflectance spectrum. 


\section{Bibliography}

H. Aasen, A. Burkart, A. Bolten, and G. Bareth. Generating 3D hyperspectral information with lightweight UAV snapshot cameras for vegetation monitoring: From camera calibration to quality assurance. ISPRS Journal of Photogrammetry and Remote Sensing, 108:245-259, 2015. ISSN 09242716. doi: 10.1016/j.isprsjprs.2015.08.002. URL http://dx.doi.org/10.1016/j.isprsjprs.2015.08.002.

M. R. Abbott and R. M. Letelier. Algorithm theoretical basis document chlorophyll fluorescence (MODIS product number 20). pages 1-42.

F. E. Abd El-Samie, M. M. Hadhoud, and S. E. El-Khamy. Image Super-Resolution and Applications. CRC Press Taylor \& Francis Group, 2012. ISBN 9781466557963.

Z. Ahmad, B. A. Franz, C. R. McClain, E. J. Kwiatkowska, J. Werdell, E. P. Shettle, and B. N. Holben. New aerosol models for the retrieval of aerosol optical thickness and normalized water-leaving radiances from the SeaWiFS and MODIS sensors over coastal regions and open oceans. Applied Optics, 49(29):5545-5560, 2010. ISSN 15394522. doi: 10.1364/AO.49.005545.

J. Aiken, S. Alvain, R. Barlow, H. Bouman, A. Bracher, R. J. W. Brewin, A. Bricaud, C. W. Brown, A. M. Ciotti, L. Clmentson, S. E. Craig, E. Devred, N. Hardman-Mountford, T. Hirata, C. Hu, T. S. Kostadinov, S. Lavender, H. Loisel, T. S. Moore, J. Morales, C. Moulin, C. B. Mouw, A. Nair, D. Raitsos, C. Roesler, S. Sathyendranath, J. D. Shutler, S. H. M., I. Soto, V. Stuart, A. Subramaniam, and J. Uitz. Detection of phytoplankton size structure by remote sensing. In S. Sathyendranath, editor, Phytoplankton Functional Types from Space, pages 71-99. International Ocean-Colour Coordinating Group, 2014. ISBN 9781896246505.

J. G. Arango and R. W. Nairn. Prediction of Optical and Non-Optical Water Quality Parameters in Oligotrophic and Eutrophic Aquatic Systems Using a Small Unmanned Aerial System. Drones, 4(1):1, 2019. ISSN 2504-446X. doi: 10.3390/drones4010001.

K. R. Arrigo and G. L. Van Dijken. Annual changes in sea-ice, chlorophyll a, and primary production in the Ross Sea, Antarctica. Deep-Sea Research Part II: Topical Studies in Oceanography, 51(1-3):117-138, 2004. ISSN 09670645. doi: 10.1016/j.dsr2.2003.04.003.

Atik Cameras. Atik Cameras Specification Tables, https://www.atik-cameras.com/ wp-content/uploads/2017/02/Atik-Specifications-Tables-Feb-17.pdf.

R. W. Austin. Optical Remote Sensing of the Oceans: BC (Before CZCS) and AC (After CZCS). In V. Barale and P. M. Schlittenhardt, editors, Ocean Colour: Theory 
and Applications in a Decade of CZCS Experience, volume 53, pages 1689-1699. ISBN 9788578110796. doi: 10.1017/CBO9781107415324.004.

S. W. Bailey, B. A. Franz, and P. J. Werdell. Estimation of near-infrared water-leaving reflectance for satellite ocean color data processing. Optics Express, 18(7):7521, 2010. ISSN 1094-4087. doi: 10.1364/oe.18.007521.

R. A. Barnes and E. F. Zalewski. Reflectance-based calibration of SeaWiFS. 1. Calibration coefficients. Applied Optics, 42(9):1629, 2003. ISSN 0003-6935. doi: 10.1364/ao.42.001629.

R. A. Barnes, W. R. Barnes, W. E. Esaias, C. R. McClain, A. W. Holmes, and T. Svitek. SeaWiFS prelaunch radiometric calibration and spectral characterization. NASA Technical Memorandum - SeaWiFS Technical Report Series, 23(October), 1994. ISSN 1522094X.

R. A. Barnes, R. E. Eplee, G. M. Schmidt, F. S. Patt, and C. R. McClain. Calibration of SeaWiFS I Direct techniques. Applied Optics, 40(36):6682, 2001. ISSN 0003-6935. doi: 10.1364/ao.40.006682.

R. A. Barnes, R. E. Eplee, F. S. Patt, H. H. Kieffer, T. C. Stone, G. Meister, J. J. Butler, and C. R. McClain. Comparison of SeaWiFS measurements of the Moon with the U.S. Geological Survey lunar model. Applied Optics, 43(31):5838-5854, 2004. ISSN 15394522. doi: 10.1364/AO.43.005838.

Basler AG. acA1920-155um - Basler ace, https://www.baslerweb.com/en/products/ cameras/area-scan-cameras/ace/aca1920-155um/\#tab=specs.

J. Behmann, K. Acebron, D. Emin, S. Bennertz, S. Matsubara, S. Thomas, D. Bohnenkamp, M. T. Kuska, J. Jussila, H. Salo, A.-k. Mahlein, and U. Rascher. Specim IQ: Evaluation of a New, Miniaturized Handheld Hyperspectral Camera and Its Application for Plant Phenotyping and Disease Detection. Sensors, 18(441):1-20, 2018. doi: 10.3390/s18020441.

M. J. Behrenfeld, R. T. O’Malley, D. A. Siegel, C. R. McClain, J. L. Sarmiento, G. C. Feldman, A. J. Milligan, P. G. Falkowski, R. M. Letelier, and E. S. Boss. Climatedriven trends in contemporary ocean productivity. Nature, 444(7120):752-755, 2006. ISSN 14764687. doi: 10.1038/nature05317.

A. Bendale and T. E. Boult. Towards Open Set Deep Networks. IEEE Conference on Computer Vision and Pattern Recognition (CVPR), pages 1563-1572, 2015.

J. L. Bezy, G. Baudin, R. Bessudo, M. A. Cutter, and D. Lobb. Medium resolution imaging spectrometer (MERIS). Proceedings of SPIE 2957, Advanced and Next-Generation Satellites II, pages 31-41, 1997. ISSN 0277786X. doi: 10.1117/12.265446.

C. Boncelet. Image noise models. In A. C. Bovik, editor, Handbook of image and video processing. 2005.

V. E. Brando, A. G. Dekker, Y. J. Park, and T. Schroeder. Adaptive semianalytical inversion of ocean color radiometry in optically complex waters. Applied Optics, 51(15):2808-2833, 2012. ISSN 0003-6935. doi: 10.1364/AO.51.002808.

A. Bricaud, A. Morel, and L. Prieur. Absorption by dissolved organic matter of the sea (yellow substance) in the UV and visible domains. Limnology and Oceanography, 26(1): 43-53, 1981. ISSN 19395590. doi: 10.4319/lo.1981.26.1.0043. 
A. Bricaud, H. Claustre, J. Ras, and K. Oubelkheir. Natural variability of phytoplanktonic absorption in oceanic waters: Influence of the size structure of algal populations. Journal of Geophysical Research: Oceans, 109(11):1-12, 2004. ISSN 21699291. doi: 10.1029/ 2004JC002419.

O. B. Brown, R. H. Evans, J. W. Brown, H. R. Gordon, R. C. Smith, and K. S. Baker. Phytoplankton blooming off the U.S. East coast: A satellite description. Science, 229 (4709):163-167, 1985. ISSN 00368075. doi: 10.1126/science.229.4709.163.

F. Cao, M. Tzortziou, C. Hu, A. Mannino, C. G. Fichot, R. Del, R. G. Najjar, and M. Novak. Remote sensing retrievals of colored dissolved organic matter and dissolved organic carbon dynamics in North American estuaries and their margins. Remote Sensing of Environment, 205(October 2017):151-165, 2018. ISSN 0034-4257. doi: 10.1016/j.rse.2017.11.014. URL https://doi.org/10.1016/j.rse.2017.11.014.

K. L. Carder, P. Reinersman, R. F. Chen, F. Muller-Karger, C. O. Davis, and M. Hamilton. AVIRIS calibration and application in coastal oceanic environments. Remote Sensing of Environment, 44(2-3):205-216, 1993. ISSN 00344257. doi: 10.1016/0034-4257(93) 90016-Q.

G. Carles, J. Downing, and A. R. Harvey. Super-resolution imaging using a camera array. Optics Letters, (March), 2014. doi: 10.1364/OL.39.001889.

C. Carrizo, A. Golovin, A. El-habashi, R. Foster, D. Gray, C. Carrizo, A. Golovin, A. Elhabashi, R. Foster, J. Bowles, and A. Gilerson. Ocean surface characterization using snapshot hyperspectral polarimetric imager. Proceedings of SPIE, 10631:1063107, 2018. doi: $10.1117 / 12.2309585$.

C. Carrizo, A. Gilerson, R. Foster, A. Golovin, and A. El-Habashi. Characterization of radiance from the ocean surface by hyperspectral imaging. Optics Express, 27(2):17501768, 2019. ISSN 1094-4087. doi: 10.1364/oe.27.001750.

A. S. Charles, C. J. Rozell, and N. Tufillaro. Sparsity Based Spectral Super-resolution and Applications to Ocean Water Color. International Geoscience and Remote Sensing Symposium, (IGARSS), Quebec, Canada, 2014.

A. P. Chase, E. Boss, I. Cetinić, and W. Slade. Estimation of Phytoplankton Accessory Pigments From Hyperspectral Reflectance Spectra: Toward a Global Algorithm. Journal of Geophysical Research: Oceans, 122(12):9725-9743, 2017. ISSN 21699291. doi: 10.1002/ 2017JC012859.

J. Chen, K. Venkataraman, D. Bakin, B. Rodricks, R. Gravelle, P. Rao, and Y. Ni. Digital camera imaging system simulation. IEEE Transactions on Electron Devices, 56(11):24962505, 2009. ISSN 00189383. doi: 10.1109/TED.2009.2030995.

M. Chin, R. A. Kahn, L. A. Remer, H. Yu, D. Rind, G. Feingold, P. K. Quinn, D. G. Streets, S. E. Schwartz, P. DeCola, and R. Halthore. Atmospheric Aerosol Properties and Climate Impacts. (January):1-115, 2009. U.S. Climate Change Science Program Synthesis and Assessment Product 2.3.

J. W. Chipman, L. G. Olmanson, and A. A. Gitelson. Remote Sensing Methods for Lake Management: A guide for resource managers and decision-makers. Developed by the North 
American Lake Management Society in collaboration with Dartmouth College, University of Minnesota, and University of Nebraska for the United States Environmental Protection Agency. 2009.

J. K. Choi, J. E. Min, J. H. Noh, T. H. Han, S. Yoon, Y. J. Park, J. E. Moon, J. H. Ahn, S. M. Ahn, and J. H. Park. Harmful algal bloom (HAB) in the East Sea identified by the Geostationary Ocean Color Imager (GOCI). Harmful Algae, 39:295-302, 2014. ISSN 15689883. doi: 10.1016/j.hal.2014.08.010. URL http://dx.doi.org/10.1016/j.hal. 2014.08.010.

Y. Choo, G. Kang, D. Kim, and S. Lee. A study on the evaluation of water-bloom using image processing. Environmental Science and Pollution Research, 25(36):36775-36780, 2018. ISSN 0944-1344. doi: 10.1007/s11356-018-3578-6.

D. K. Clark, M. A. Yarbrough, M. Feinholz, S. Flora, W. Broenkow, Y. S. Kim, B. C. Johnson, S. W. Brown, M. Yuen, and J. L. Mueller. MOBY, A Radiometric Buoy for Performance Monitoring and Vicarious Calibration of Satellite Ocean Color Sensors: Measurement and Data Analysis Protocols. SeaWiFs Postlaunch Technical Report Series, VI (Rev. 4):3-34, 2003.

G. L. Clarke, G. C. Ewing, and C. J. Lorenzen. Spectra of Backscattered Light from the Sea Obtained from Aircraft as a Measure of Chlorophyll Concentration Abstract. Science, 167(3921):1119-1121, 1970.

P. G. Coble. Marine optical biogeochemistry: The chemistry of ocean color. Chemical Reviews, 107(2):402-418, 2007. ISSN 00092665. doi: 10.1021/cr050350+.

Cuber GmbH. S185 Hardware Operation Guide, https://cubert-gmbh.com/wp-content/ uploads/hardware-operation-guide-firefleye.pdf.

T.-W. Cui, Q.-J. Song, J.-W. Tang, and J. Zhang. Spectral variability of sea surface skylight reflectance and its effect on ocean color. Optics Express, 21(21):24929-24941, 2013. ISSN 1094-4087. doi: 10.1364/oe.21.024929.

M. Dalponte, L. Bruzzone, and D. Gianelle. Tree species classification in the Southern Alps based on the fusion of very high geometrical resolution multispectral/hyperspectral images and LiDAR data. Remote Sensing of Environment, 123:258-270, 2012. ISSN 00344257. doi: 10.1016/j.rse.2012.03.013. URL http://dx.doi.org/10.1016/j.rse.2012.03.013.

C. Davis, J. Bowles, R. Leathers, D. Korwan, T. V. Downes, W. Snyder, W. Rhea, W. Chen, J. Fisher, P. Bissett, and R. A. Reisse. Ocean PHILLS hyperspectral imager: design, characterization, and calibration. Optics Express, 10(4):210-221, 2002. ISSN 1094-4087. doi: 10.1364/OE.10.000210.

C. O. Davis. GOES-R and the Hyperspectral Environmental Sensor (HES) Suite.

C. O. Davis, M. Kavanaugh, R. M. Letelier, W. P. Bissett, and D. Kohler. Spatial and spectral resolution considerations for imaging coastal waters. Proceedings of SPIE, 6680 (4):66800P, 2007. ISSN 0277786X. doi: 10.1117/12.734288.

M. Defoin-Platel and M. Chami. How ambiguous is the inverse problem of ocean color in coastal waters? Journal of Geophysical Research: Oceans, 112(3):1-16, 2007. ISSN 21699291. doi: 10.1029/2006JC003847. 
T. Dickey, M. Lewis, and G. Chang. Optical oceanography: Recent advances and future directions using global remote sensing and in situ observations. Reviews of Geophysics, 44(1):1-39, 2006. ISSN 87551209. doi: 10.1029/2003RG000148.

H. M. Dierssen, R. C. Zimmerman, R. A. Leathers, T. V. Downes, and C. O. Davis. Ocean color remote sensing of seagrass and bathymetry in the Bahamas Banks by high-resolution airborne imagery. Limnology and Oceanography, 48(1 II):444-455, 2003. ISSN 00243590. doi: 10.4319/lo.2003.48.1_part_2.0444.

H. M. Dierssen, A. Chlus, and B. Russell. Hyperspectral discrimination of floating mats of seagrass wrack and the macroalgae Sargassum in coastal waters of Greater Florida Bay using airborne remote sensing. Remote Sensing of Environment, 167:247-258, 2015. ISSN 00344257. doi: 10.1016/j.rse.2015.01.027. URL http://dx.doi.org/10.1016/j . rse.2015.01.027.

P. D'Odorico, E. Alberti, and M. E. Schaepman. In-flight spectral performance monitoring of the Airborne Prism Experiment. Applied Optics, 49(16):3082-3091, 2010. ISSN 15394522. doi: 10.1364/AO.49.003082.

S. C. Doney. Plankton in a warmer world. Nature, 444:695-696, 2006.

N. E. Dorsey. Properties of Ordinary Water-Substance: In all its Phases: Water-vapor, Water, and all the Ices, 1940.

J. Edling. Tetracam Inc., 21601 Devonshire St. \# 310, Chatsworth, CA 91311 (personal communication, 2019).

K. Ehmann, C. Kelleher, and L. E. Condon. Monitoring turbidity from above: Deploying small unoccupied aerial vehicles to image in-stream turbidity. Hydrological Processes, 33 (6):1013-1021, 2019. ISSN 10991085. doi: 10.1002/hyp.13372.

S. Ekercin. Water Quality Retrievals from High Resolution Ikonos Multispectral Imagery: A Case Study in Istanbul, Turkey. Water, Air and Soil Pollution, 183(1-4):239-251, 2007. doi: $10.1007 / \mathrm{s} 11270-007-9373-5$.

O. Engelsen, E. N. Hegseth, H. Hop, E. Hansen, and S. Falk-Petersen. Spatial variability of chlorophyll-a in the Marginal Ice Zone of the Barents Sea, with relations to sea ice and oceanographic conditions. Journal of Marine Systems, 35(1-2):79-97, 2002. ISSN 09247963. doi: 10.1016/S0924-7963(02)00077-5.

P. G. Falkowski and J. A. Raven. Aquatic Photosynthesis. 2007. ISBN 9780691115504. doi: $10.1515 / 9781400849727$.

L. Fang, H. Zhuo, and S. Li. Super-resolution of hyperspectral image via superpixel-based sparse representation. Neurocomputing, 273:171-177, 2018. ISSN 18728286. doi: 10.1016/ j.neucom.2017.08.019. URL https://doi.org/10.1016/j.neucom.2017.08.019.

J. E. Farrell, P. B. Catrysse, and B. A. Wandell. Digital camera simulation. Applied Optics, 51(4):80-90, 2012. ISSN 15394522. doi: 10.1364/AO.51.000A80.

Federal Aviation Administration (FAA). Remote Pilot in Command Certification and Responsibilities. (Part 107):1-3, 2016a. URL http://www.faa.gov/uas/media/Part_107_ Summary.pdf. 
Federal Aviation Administration (FAA). Code of Federal Regulations (14 CFR) part 107. 2016b. URL https://www.faa.gov/documentlibrary/media/advisory_circular/ac_ 107-2.pdf.

L. Feng, C. Hu, B. B. Barnes, A. Mannino, A. K. Heidinger, K. Strabala, and L. T. Iraci. Cloud and Sun-glint statistics derived from GOES and MODIS observations over the Intra-Americas Sea for GEO-CAPE mission planning. Journal of Geophysical Research: Atmospheres, 122:1725-1745, 2017. doi: 10.1002/2016JD025372.

C. G. Fichot, B. D. Downing, B. A. Bergamaschi, L. Windham-Myers, M. MarvinDipasquale, D. R. Thompson, and M. M. Gierach. High-Resolution Remote Sensing of Water Quality in the San Francisco Bay-Delta Estuary. Environmental Science and Technology, 50(2):573-583, 2016. ISSN 15205851. doi: 10.1021/acs.est.5b03518.

J. Fisher and J. Antomades. A hyperspectral imaging sensor for the coastal environment. Proceedings of SPIE 3482, International Optical Design Conference 1998, pages 179-186, 1998.

D. T. Fitch and J. K. Moore. Wind speed influence on phytoplankton bloom dynamics in the Southern Ocean Marginal Ice Zone. Journal of Geophysical Research: Oceans, 112(8): 1-13, 2007. ISSN 21699291. doi: 10.1029/2006JC004061.

S. Flora. Listing of MOBY Spectral Data. https://www.star.nesdis.noaa.gov/socd/ moby/filtered_spec/.

K. Fotiadou, G. Tsagkatakis, and P. Tsakalides. Spectral Super Resolution of Hyperspectral Images via Coupled Dictionary Learning. IEEE Transactions on Geoscience and Remote Sensing, 57(5):2777-2797, 2019. ISSN 01962892. doi: 10.1109/TGRS.2018.2877124.

B. Fougnie, R. Frouin, P. Lecomte, and P.-Y. Deschamps. Reduction of skylight reflection effects in the above-water measurement of diffuse marine reflectance: comment. Applied Optics, 38(18):1379-1381, 1999. ISSN 0003-6935. doi: 10.1364/AO.39.001379.

B. A. Franz and P. J. Werdell. A generalized framework for modeling of inherent optical properties in ocean remote sensing applications. Proceedings of Ocean Optics 2010, (October):1-13, 2010.

B. A. Franz, S. W. Bailey, P. J. Werdell, and C. R. McClain. Sensor-independent approach to the vicarious calibration of satellite ocean color radiometry. Applied Optics, 46(22): 5068-5082, 2007. ISSN 15394522. doi: 10.1364/AO.46.005068.

L. Galvis, H. Arguello, and G. R. Arce. Coded aperture design in mismatched compressive spectral imaging. Applied Optics, 54(33):9875-9882, 2015.

B.-C. Gao, M. J. Montes, Z. Ahmad, and C. O. Davis. Atmospheric correction algorithm for hyperspectral remote sensing of ocean color from space. Applied Optics, 39(6):887, 2000. ISSN 0003-6935. doi: 10.1364/аo.39.000887.

S. P. Garaba and H. M. Dierssen. Hyperspectral ultraviolet to shortwave infrared characteristics of marine-harvested, washed-ashore and virgin plastics. Earth System Science Data, 12(1):77-86, 2020. ISSN 18663516. doi: 10.5194/essd-12-77-2020. 
U. B. Gewali, S. T. Monteiro, and E. Saber. Spectral super-resolution with optimized bands. Remote Sensing, 11(14):1-24, 2019. ISSN 20724292. doi: 10.3390/rs11141648.

M. Gholizadeh, A. Melesse, and L. Reddi. A Comprehensive Review on Water Quality Parameters Estimation Using Remote Sensing Techniques. Sensors, 16(1298):1-43, 2016. ISSN 1424-8220. doi: 10.3390/s16081298. URL http://www.mdpi.com/1424-8220/16/ 8/1298.

A. Gilerson, C. Carrizo, R. Foster, and T. Harmel. Variability of the reflectance coefficient of skylight from the ocean surface and its implications to ocean color. Optics Express, 26 (8):9615-9633, 2018.

H. R. Gordon and W. M. Balch. MODIS Detached Coccolith Concentration Algorithm Theoretical Basis Document. Ocean Color web page, pages 1-27, 1999.

H. R. Gordon and W. R. McCluney. Estimation of the Depth of Sunlight Penetration in the Sea for Remote Sensing. Applied Optics, 14(2):413, 1975. ISSN 0003-6935. doi: 10.1364/ao.14.000413.

H. R. Gordon and A. Y. Morel. Remote Assessment of Ocean Color for Interpretation of Satellite Visible Imagery: A Review. Springer-Verlag, 1983. ISBN 0387909230.

H. R. Gordon and K. J. Voss. MODIS normalized water-leaving radiance algorithm theoretical basis document. pages 1-96, 2004.

H. R. Gordon and M. Wang. Retrieval of water-leaving radiance and aerosol optical thickness over the oceans with SeaWiFS: a preliminary algorithm. Applied Optics, 33(3):443, 1994. ISSN 0003-6935. doi: 10.1364/ao.33.000443.

H. R. Gordon, D. K. Clark, J. L. Mueller, and W. A. Hovis. Phytoplankton Pigments from the Nimbus-7 Coastal Zone Color Scanner: Comparisons with Surface Mesurements. Science, 210(3):63-66, 1980.

H. R. Gordon, T. Du, and T. Zhang. Remote sensing of ocean color and aerosol properties: resolving the issue of aerosol absorption. Applied Optics, 36(33):8670, 1997. ISSN 00036935. doi: 10.1364/ao.36.008670.

R. Gordon, O. B. Brown, H. Evans, W. Brown, C. Smith, K. Baker, and D. K. Clark. A Semianalytic Radiance Model of Ocean Color. Journal of Geophysical Research, 93(9): 10909-10924, 1988.

M. Gosselin, M. Levasseur, P. A. Wheeler, R. A. Horner, and B. C. Booth. New measurements of phytoplankton and ice algal production in the Arctic Ocean. Deep-Sea Research Part II, 44(8):1623-1644, 1997. ISSN 09670645. doi: 10.1016/S0967-0645(97)00054-4.

R. D. Gow, D. Renshaw, K. Findlater, L. Grant, S. J. McLeod, J. Hart, and R. L. Nicol. A comprehensive tool for modeling CMOS image-sensor-noise performance. IEEE Transactions on Electron Devices, 54(6):1321-1329, 2007. ISSN 00189383. doi: 10.1109/TED.2007.896718.

R. O. Green, M. Eastwood, I. McCubbin, C. Chovit, J. Raney, and J. Holbrook. Overview of AVIRIS Acquisitions in Argentina as Part of the NM EO-1 Campaign in 2001, 2001a. URL http://ntrs .nasa.gov/search.jsp?R=20080009654. 
R. O. Green, B. Pavri, and J. Boardman. On-Orbit calibration of an ocean color sensor with an underflight of the Airborne Visible/Infrared Imaging Spectrometer (AVIRIS). Elsevier Science, 28(1):133-142, 2001b.

B. Griffin, M. K. Griffin, and H.-h. K. Burke. Compensation of Hyperspectral Data for Atmospheric Effects. Lincoln Laboratory Journal, 14(1):29-54, 2003.

M. R. Grose and A. McMinn. Algal biomass in East Antarcic pack ice: how much is in the east? In A. Huiskes, W. Gieskes, J. Rozema, R. Schorno, S. v. d. Vies, and W. Wolff, editors, Antarctic biology in a global context, pages 21--25. 1003 Backhuys Publishers, 2003.

T. T. Guimarães, M. R. Veronez, E. C. Koste, E. M. Souza, D. Brum, L. Gonzaga, and F. F. Mauad. Evaluation of regression analysis and neural networks to predict total suspended solids in water bodies from unmanned aerial vehicle images. Sustainability (Switzerland), 11(9), 2019. ISSN 20711050. doi: 10.3390/su11092580.

D. Gurlin, A. A. Gitelson, and W. J. Moses. Remote estimation of chl-a concentration in turbid productive waters - Return to a simple two-band NIR-red model? Remote Sensing of Environment, 115(12):3479-3490, 2011. ISSN 00344257. doi: 10.1016/j.rse.2011.08.011. URL http://dx.doi.org/10.1016/j.rse.2011.08.011.

Hamamatsu Photonics K. K. NMOS linear image sensor S3901/S3904 series, https://www . hamamatsu.com/resources/pdf/ssd/s3901-128q_etc_kmpd1036e.pdf.

M. K. Hamilton, C. O. Davis, S. H. Pilorz, W. J. Rhea, and K. L. Carder. Examination of chlorophyll distribution in Lake Tahoe, using the Airborne Visible and Infrared Imaging Spectrometer (AVIRIS), 1991.

Headwall Photonics, Inc. Nano-Hyperspec Hyperspectral Imaging Sensor, https:// geo-konzept.de/wp-content/uploads/2019/12/NanoHyperspec0118_prospect.pdf.

F. E. Hoge, C. W. Wright, and R. N. Swift. Radiance-ratio algorithm wavelengths for remote oceanic chlorophyll determination. Applied Optics, 26:2082-2094, 1987. ISSN 0003-6935. doi: 10.1364/AO.26.002082.

P. M. Holligan, M. Viollier, D. S. Harbour, P. Camus, and M. Champagne-Philippe. Satellite and Ship Studies of Coccolithophore Production Along a Continental Shelf Edge. Nature, 304(July):339-342, 1983.

E. Honkavaara, H. Saari, J. Kaivosoja, I. Pölönen, T. Hakala, P. Litkey, J. Mäkynen, and L. Pesonen. Processing and assessment of spectrometric, stereoscopic imagery collected using a lightweight UAV spectral camera for precision agriculture. Remote Sensing, 5(10): 5006-5039, 2013. ISSN 20724292. doi: 10.3390/rs5105006.

C. Hu, K. L. Carder, and F. E. Muller-Karger. How precise are SeaWiFS ocean color estimates? Implications of digitization-noise errors. Remote Sensing of Environment, 76 (2):239-249, 2001. ISSN 00344257. doi: 10.1016/S0034-4257(00)00206-6.

C. Hu, L. Feng, Z. Lee, C. O. Davis, A. Mannino, C. R. McClain, and B. A. Franz. Dynamic range and sensitivity requirements of satellite ocean color sensors: learning from the past. Applied Optics, 51(25):6045-6062, 2012. ISSN 0003-6935. doi: 10.1364/AO.51.006045. 
P. D. Hunter, A. N. Tyler, L. Carvalho, G. A. Codd, and S. C. Maberly. Hyperspectral remote sensing of cyanobacterial pigments as indicators for cell populations and toxins in eutrophic lakes. Remote Sensing of Environment, 114(11):2705-2718, 2010. ISSN 00344257. doi: 10.1016/j.rse.2010.06.006. URL http://dx.doi.org/10.1016/j.rse. 2010.06 .006 .

T. Huth-Fehre, R. Feldhoff, F. Kowol, H. Freitag, S. Kuttler, B. Lohwasser, and M. Oleimeulen. Remote sensor systems for the automated identification of plastics. Journal of Near Infrared Spectroscopy, 11:7-11, 1998.

IGOS. A Coastal Theme for the IGOS Partnership - For the Monitoring of our Environment from Space and from Earth. UNESCO, pages 1-67, 2006.

International Ocean-Colour Coordinating Group. Mission requirements for future oceancolour sensors. In Reports of International Ocean-Colour Coordinating Group. No. 13. 2012a.

International Ocean-Colour Coordinating Group. Ocean-Colour Observations from a Geostationaary Orbi. In Reports of International Ocean-Colour Coordinating Group. No. 12. 2012b.

International Ocean-Colour Coordinating Group. 2017 International Ocean Colour Science Meeting (IOCS-2017). 2017 International Ocean Colour Science Meeting (IOCS-2017), (May):1-59, 2017.

J. Ishizaka. Coupling of Coastal Zone Color Scanner data to a physical-biological model of the southeastern US continental shelf ecosystem 1. CZCS Data Description and Lagrangian Particle Tracing Experiments. Journal of Geophysical Research, 95(11):167-181, 1990. ISSN 01480227. doi: 10.1029/jc095ic11p20183.

J. R. Janesick. Photon Transfer. SPIE, 2007.

N. G. Jerlov. Marine Optics. Elsevier Scientific Pub. Co., 1976.

Jet Propulsion Laboratory. HyspIRI Final Report. NASA HyspIRI Final Report, (September):91, 2018. URL https://hyspiri.jpl.nasa.gov/downloads/reports_ whitepapers/HyspIRI_FINAL_Report_10ctober2018_20181005a.pdf.

D. J. Keith, B. A. Schaeffer, R. S. Lunetta, R. W. G. Jr, K. Rocha, and D. J. Cobb. Remote sensing of selected water-quality indicators with the hyperspectral imager for the coastal ocean (HICO) sensor. International Journal of Remote Sensing, 35(9):2927-2962, 2014. ISSN 0143-1161. doi: 10.1080/01431161.2014.894663. URL http://dx.doi.org/ $10.1080 / 01431161.2014 .894663$.

M. Kim, J. Y. Park, Y. I. Kopilevich, G. H. Tuell, and W. D. Philpot. Correction for reflected sky radiance in low-altitude coastal hyperspectral images. Applied Optics, 52 (32):7732-7744, 2013. ISSN 0003-6935. doi: 10.1364/AO.52.007732. URL http://www . opticsinfobase.org/ao/abstract.cfm?uri=ao-52-32-7732.

S. P. Kim, N. K. Bose, and H. M. Valenzuela. Recursive Reconstruction of High Resolution Image From Noisy Undersampled Multiframes. IEEE Transactions on Acoustics, Speech, and Signal Processing, 38(6):1013-1027, 1990. ISSN 00963518. doi: 10.1109/29.56062. 
J. T. O. Kirk. Light and Photosynthesis in Aquatic Ecosystems. Cambridge University Press, 2nd edition, 1994.

C. Kislik, I. Dronova, and M. Kelly. UAVs in Support of Algal Bloom Research: A Review of Current Applications and Future Opportunities. Drones, 2(4):35, 2018. ISSN 2504-446X. doi: $10.3390 /$ drones2040035.

K. Kitano, T. Funatomi, R. Yasukuni, K. Tanaka, H. Kubo, Y. Hosokawa, and Y. Mukaigawa. Spectral Super-resolution by Image Sensor Tilting. In Imaging and Applied Optics Congress, pages 1-2, 2020.

V. V. Klemas. Coastal and Environmental Remote Sensing from Unmanned Aerial Vehicles: An Overview. Journal of Coastal Research, 315:1260-1267, 2015. ISSN 0749-0208. doi: 10.2112/JCOASTRES-D-15-00005.1. URL http://www .bioone.org/doi/10.2112/ JCOASTRES-D-15-00005.1.

T. Konishi, Y. Yamasaki, and T. Nagashima. Super spectral resolution beyond pixel Nyquist limits on multi-channel spectrometer. Optics Express, 24(23):26583, 2016. ISSN 1094-4087. doi: 10.1364/oe.24.026583.

H. J. Kramer. PRISM (Portable Remote Imaging Spectrometer) airborne instrument. URL https://directory.eoportal.org/web/eoportal/airborne-sensors/prism

T. Kutser. Quantitative detection of chlorophyll in cyanobacterial blooms by satellite remote sensing. Limnology and Oceanography, 49(6):2179-2189, 2004. ISSN 00243590. doi: 10.4319/lo.2004.49.6.2179.

Y. S. Kwon, J. C. Pyo, Y. H. Kwon, H. Duan, K. H. Cho, and Y. Park. Drone-based hyperspectral remote sensing of cyanobacteria using vertical cumulative pigment concentration in a deep reservoir. Remote Sensing of Environment, 236(January 2019):111517, 2020. ISSN 00344257. doi: 10.1016/j.rse.2019.111517. URL https://doi.org/10.1016/ j.rse.2019.111517.

M. D. Larson, A. Simic Milas, R. K. Vincent, and J. E. Evans. Multi-depth suspended sediment estimation using high-resolution remote-sensing UAV in Maumee River, Ohio. International Journal of Remote Sensing, 39(15-16):5472-5489, 2018. ISSN 13665901. doi: 10.1080/01431161.2018.1465616. URL https://doi.org/10.1080/01431161.2018. 1465616.

H. Lee, G. J. Hill, B. L. Vattiat, M. P. Smith, and M. Haeuser. Facility calibration unit of Hobby Eberly Telescope wide field upgrade. 8444:84444J, 2012. ISSN 0277786X. doi: 10.1117/12.926736. URL http://proceedings . spiedigitallibrary.org/proceeding. aspx?doi=10.1117/12.926736.

Z. Lee and K. L. Carder. Effect of spectral band numbers on the retrieval of water column and bottom properties from ocean color data. Applied Optics, 41(12):2191-2201, 2002. ISSN 0003-6935. doi: 10.1364/AO.41.002191. URL http://www.ncbi.nlm.nih.gov/pubmed/ 12003210 .

Z. Lee, K. L. Carder, and R. A. Arnone. Deriving inherent optical properties from water color: a multiband quasi-analytical algorithm for optically deep waters. Applied Optics, 41(27):5755, 2002. ISSN 0003-6935. doi: 10.1364/ao.41.005755. 
Z. Lee, Y.-H. Ahn, C. Mobley, and R. Arnone. Removal of surface-reflected light for the measurement of remote-sensing reflectance from an above-surface platform. Optics Express, 18(25):26313-26324, 2010. ISSN 1094-4087. doi: 10.1364/OE.18.026313.

Z. Lee, S. Shang, C. Hu, and G. Zibordi. Spectral interdependence of remote-sensing reflectance and its implications on the design of ocean color satellite sensors. Applied Optics, 53(15):3301-3310, 2014. ISSN 0003-6935. doi: 10.1364/AO.53.003301. URL https://www. osapublishing.org/abstract.cfm?URI=ao-53-15-3301.

J. Lekki, E. Deutsch, M. Sayers, K. Bosse, R. Anderson, R. Tokars, and R. Sawtell. Determining remote sensing spatial resolution requirements for the monitoring of harmful algal blooms in the Great Lakes. Journal of Great Lakes Research, 45(3):434-443, 2019. ISSN 03801330. doi: 10.1016/j.jglr.2019.03.014. URL https://doi.org/10.1016/j. jglr.2019.03.014.

I. Levin, E. Levina, G. Gilbert, and S. Stewart. Role of sensor noise in hyperspectral remote sensing of natural waters: Application to retrieval of phytoplankton pigments. Remote Sensing of Environment, 95(2):264-271, 2005. ISSN 00344257. doi: 10.1016/j.rse.2005. 01.001 .

I. M. Levin and E. Levina. Effect of atmospheric interference and sensor noise in retrieval of optically active materials in the ocean by hyperspectral remote sensing. Applied Optics, 46(28):6896-6906, 2007. ISSN 15394522. doi: 10.1364/AO.46.006896.

L. Liebel and M. Körner. Single-image super resolution for multispectral remote sensing data using convolutional neural networks. International Archives of the Photogrammetry, Remote Sensing and Spatial Information Sciences - ISPRS Archives, 41(July):883-890, 2016. ISSN 16821750. doi: 10.5194/isprsarchives-XLI-B3-883-2016.

E. M. Louchard, R. P. Reid, F. C. Stephens, C. O. Davis, R. A. Leathers, and T. V. Downes. Optical remote sensing of benthic habitats and bathymetry in coastal environments at Lee Stocking Island, Bahamas: A comparative spectral classification approach. Limnology and Oceanography, 48(1 II):511-521, 2003. ISSN 00243590. doi: 10.4319/lo.2003.48.1_part_ 2.0511 .

R. Lu. Detection if Bruises on Apples Using Near-Infrared Hyperspectral Imaging. Transactions of the ASAE, 46(2):523-530, 2003.

B. Lubac, H. Loisel, N. Guiselin, R. Astoreca, L. F. Artigas, and X. Mériaux. Hyperspectral and multispectral ocean color inversions to detect Phaeocystis globosa blooms in coastal waters. Journal of Geophysical Research: Oceans, 113(6):1-17, 2008. ISSN 21699291. doi: 10.1029/2007JC004451.

R. L. Lucke, M. Corson, N. R. McGlothlin, S. D. Butcher, D. L. Wood, D. R. Korwan, R. R. Li, W. A. Snyder, C. O. Davis, and D. T. Chen. Hyperspectral Imager for the Coastal Ocean: instrument description and first images. Applied Optics, 50(11):1501-1516, 2011. ISSN 0003-6935. doi: 10.1364/AO.50.001501. URL https://www.osapublishing.org/ abstract.cfm?URI=ao-50-11-1501.

K. M. Luis, J. E. Rheuban, M. T. Kavanaugh, D. M. Glover, J. Wei, Z. Lee, and S. C. Doney. Capturing coastal water clarity variability with Landsat 8. Marine Pollution Bulletin, 145 
(December 2018):96-104, 2019. ISSN 18793363. doi: 10.1016/j.marpolbul.2019.04.078. URL https://doi.org/10.1016/j.marpolbul.2019.04.078.

R. S. Lunetta, J. F. Knight, H. W. Paerl, J. J. Streicher, B. L. Peierls, T. Gallo, J. G. Lyon, T. H. Mace, and C. P. Buzzelli. Measurement of water colour using AVIRIS imagery to assess the potential for an operational monitoring capability in the Pamlico Sound Estuary, USA. International Journal of Remote Sensing, 30(13):3291-3314, 2009. ISSN 13665901. doi: 10.1080/01431160802552801.

C. Ma, X. Cao, X. Tong, Q. Dai, and S. Lin. Acquisition of High Spatial and Spectral Resolution Video with a Hybrid Camera System. International Journal of Computer Vision, 110(2):141-155, 2014. ISSN 15731405. doi: 10.1007/s11263-013-0690-4.

A. Malinka, E. Zege, G. Heygster, and L. Istomina. Reflective properties of white sea ice and snow. Cryosphere, 10(6):2541-2557, 2016. ISSN 19940424. doi: 10.5194/tc-10-2541-2016.

T. Malone, M. Azzaro, A. Bode, E. Brown, R. Duce, D. Kamykowski, S. H. Kang, Y. Kedong, M. Thorndyke, J. Wang, C. Park, H. Calumpong, and P. Eghtesadi. Chapter 6. Primary Production, Cycling of Nutrients, Surface Layer and Plankton. pages 1-67, 2016.

S. Maritorena, D. A. Siegel, and A. R. Peterson. Optimization of a semianalytical ocean color model for global-scale applications. Applied Optics, 41(15):2705, 2002. ISSN 00036935. doi: 10.1364/AO.41.002705. URL https://www.osapublishing.org/abstract. cfm?URI=ao-41-15-2705.

R. A. Massom, S. E. Stammerjohn, R. C. Smith, M. J. Pook, R. A. Iannuzzi, N. Adams, D. G. Martinson, M. Vernet, W. R. Fraser, L. B. Quetin, R. M. Ross, Y. Massom, and H. R. Krouse. Extreme anomalous atmospheric circulation in the West Antarctic Peninsula region in austral spring and summer 2001/02, and its profound impact on sea ice and biota. Journal of Climate, 19(15):3544-3571, 2006. ISSN 08948755. doi: 10.1175/JCLI3805.1.

D. Mauruschat, B. Plinke, J. Aderhold, J. Gunschera, P. Meinlschmidt, and T. Salthammer. Application of near-infrared spectroscopy for the fast detection and sorting of wood-plastic composites and waste wood treated with wood preservatives. Wood Science and Technology, 50(2):313-331, 2016. ISSN 00437719. doi: 10.1007/s00226-015-0785-x.

C. R. McClain. Review of Major CZCS Applications: U.S. Case Studies. In V. Barale and P. M. Schlittenhardt, editors, Ocean Colour: Theory and Applications in a Decade of CZCS Experience, volume 53, pages 17-32. ISBN 9788578110796. doi: 10.1017/CBO9781107415324.004.

C. R. McClain. A Decade of Satellite Ocean Color Observations. Annual Review of Marine Science, 1(1):19-42, 2009. ISSN 1941-1405. doi: 10.1146/annurev.marine.010908.163650.

C. R. McClain, M. L. Cleave, C. Gene, W. W. Gregg, S. B. Hooker, and N. Kuring. Science Quality SeaWiFS Data for Global Biosphere. Sea Technology, 39:1-7, 1998.

C. R. McClain, G. Meister, and B. Monosmith. Satellite ocean color sensor design concepts and performance requirements, volume 47. 2014. ISBN 9780124170117. doi: 10.1016/ B978-0-12-417011-7.00005-2. 
A. McMinn, A. Pankowskii, C. Ashworth, R. Bhagooli, P. Ralph, and K. Ryan. In situ net primary productivity and photosynthesis of Antarctic sea ice algal, phytoplankton and benthic algal communities. Marine Biology, 157(6):1345-1356, 2010. doi: 10.1007/ s00227-010-1414-8.

A. Meams, M. Stekoll, K. Hall, C. B. Krause, M. Watson, and M. Atkinson. Biological and Ecological Effects of Wastewater Discharges from Cruise Ships in Alaska. Oceans 2003. Celebrating the Past ... Teaming Toward the Future, 2:737-747, 2003.

G. Meister, Y. Zong, and C. R. McClain. Derivation of the MODIS Aqua Point-Spread Function ocean color bands. Earth Observing Systems XIII, 7081(May 2014):70811F, 2008. doi: $10.1117 / 12.796980$.

J. Meola. Examining the impact of spectral uncertainty on hyperspectral data exploitation. Proceedings of SPIE, 10644(May 2018):1 — 12, 2018. ISSN 1996756X. doi: 10.1117/12. 2303725.

J. Meola, M. T. Eismann, R. L. Moses, and J. N. Ash. Modeling and estimation of signaldependent noise in hyperspectral imagery. Applied Optics, 50(21):3829-3846, 2011.

MicaSense. RedEdge-M/MX Manual: Mapping Operations, https://support.micasense. com/hc/en-us/articles/115003684453-RedEdge-M-MX-Manual-Mapping-Operations . a.

MicaSense. RedEdge-MX, https://www.micasense.com/rededge-mx . b.

S. Mishra and D. R. Mishra. Normalized difference chlorophyll index: A novel model for remote estimation of chlorophyll-a concentration in turbid productive waters. Remote Sensing of Environment, 117:394-406, 2012. ISSN 00344257. doi: 10.1016/j.rse.2011.10. 016. URL http://dx.doi.org/10.1016/j.rse.2011.10.016.

C. Mobley. Ocean Optics Web Book: Light from the Sun. In Ocean Optics Web Book. 2020. URL https://oceanopticsbook.info/index.php/view/light-and-radiometry/ level-2/light-from-the-sun.

C. Mobley, B. Monosmith, and J. Werdell. Ocean Optics Web Book: Counting Photons, 2016a. URL http://www.oceanopticsbook.info/view/remote_sensing/level_ 2/counting_photons

C. D. Mobley. Estimation of the remote-sensing reflectance from above-surface measurements. Applied Optics, 38(36):7442-7455, 1999. ISSN 0003-6935. doi: 10.1364/AO.38. 007442. URL https://www . osapublishing.org/abstract.cfm?URI=ao-38-36-7442.

C. D. Mobley. Modeling Sea Surfaces: A Tutorial on Fourier Transform Techniques. Technical report, Sequoia Scientific, Inc., 2016.

C. D. Mobley and L. K. Sundman. HydroLight 5 Ecolight 5 user's guide. Sequoia Scientific, Inc., 2008.

C. D. Mobley, J. Werdell, B. Franz, Z. Ahmad, and S. Bailey. Atmospheric Correction for Satellite Ocean Color Radiometry A Tutorial and Documentation of the Algorithms Used by the NASA Ocean Biology Processing Group. 2016b. 
C. D. Mobley, J. Werdell, B. Franz, Z. Ahmad, and S. Bailey. Atmospheric Correction for Satellite Ocean Color Radiometry. NASA Technical Memorandum, 201621755(NASA/TM-2016-217551):1-73, 2016c. URL https://oceancolor.gsfc.nasa. gov/docs/technical/.

A. Morel and L. Prieur. Analysis of variations in ocean color. Limnology and Oceanography, 22(4):709-722, 1977. ISSN 19395590. doi: 10.4319/lo.1977.22.4.0709.

M. Moroni, A. Mei, A. Leonardi, E. Lupo, and F. La Marca. PET and PVC separation with hyperspectral imagery. Sensors, 15(1):2205-2227, 2015. ISSN 14248220. doi: 10.3390/ s150102205.

W. J. Moses, J. H. Bowles, R. L. Lucke, and M. R. Corson. Impact of signal-to-noise ratio in a hyperspectral sensor on the accuracy of biophysical parameter estimation in case II waters. Optics Express, 20(4):4309-4330, 2012a. doi: 10.1117/12.734288.

W. J. Moses, A. A. Gitelson, R. L. Perk, D. Gurlin, D. C. Rundquist, B. C. Leavitt, T. M. Barrow, and P. Brakhage. Estimation of chlorophyll-a concentration in turbid productive waters using airborne hyperspectral data. Water Research, 46(4):993-1004, 2012b. ISSN 00431354. doi: 10.1016/j.watres.2011.11.068. URL http://dx.doi.org/10.1016/ j.watres.2011.11.068.

W. J. Moses, J. H. Bowles, and M. R. Corson. Expected improvements in the quantitative remote sensing of optically complex waters with the use of an optically fast hyperspectral spectrometer - a modeling study. Sensors (Switzerland), 15(3):6152-6173, 2015. ISSN 14248220. doi: $10.3390 / \mathrm{s} 150306152$.

W. J. Moses, S. G. Ackleson, J. W. Hair, C. A. Hostetler, and W. D. Miller. Spatial scales of optical variability in the coastal ocean: Implications for remote sensing and in situ sampling. Journal of Geophysical Research: Oceans, 121:4194-4208, 2016. doi: 10.1002/2016JC011767.Received.

K. M. G. Mostofa, C.-q. Liu, M. A. Mottaleb, G. Wan, H. Ogawa, D. Vione, T. Yoshioka, F. engchang $\mathrm{Wu}$, and 1. Dissolved Organic Matter in Natural Waters. In Photobiogeochemistry of Organic Matter, volume 179, pages 1309-1326. 2009. ISBN 0029-8549. doi: 10.1007/978-3-642-32223-5.

P. Mouroulis and R. O. Green. Review of high fidelity imaging spectrometer design for remote sensing. Optical Engineering, 57(04):1-19, 2018. ISSN 1560-2303. doi: 10.1117/1. oe.57.4.040901.

P. Mouroulis, R. O. Green, and T. G. Chrien. Design of pushbroom imaging spectrometers for optimum recovery of spectroscopic and spatial information. Applied Optics, 39(13): 2210, 2000. ISSN 0003-6935. doi: 10.1364/ao.39.002210.

P. Mouroulis, B. Van Gorp, R. O. Green, H. Dierssen, D. W. Wilson, M. Eastwood, J. Boardman, B.-C. Gao, D. Cohen, B. Franklin, F. Loya, S. Lundeen, A. Mazer, I. McCubbin, D. Randall, B. Richardson, J. I. Rodriguez, C. Sarture, E. Urquiza, R. Vargas, V. White, and K. Yee. Portable Remote Imaging Spectrometer coastal ocean sensor: design, characteristics, and first flight results. Applied Optics, 53(7):1363, 2014. ISSN 00036935. doi: 10.1364/AO.53.001363. URL https://www.osapublishing.org/abstract. cfm?URI=ao-53-7-1363. 
C. B. Mouw, S. Greb, D. Aurin, P. M. DiGiacomo, Z. Lee, M. Twardowski, C. Binding, C. $\mathrm{Hu}, \mathrm{R}$. Ma, T. Moore, W. Moses, and S. E. Craig. Aquatic color radiometry remote sensing of coastal and inland waters: Challenges and recommendations for future satellite missions. Remote Sensing of Environment, 160:15-30, 2015. ISSN 00344257. doi: 10. 1016/j.rse.2015.02.001. URL http://dx.doi.org/10.1016/j.rse.2015.02.001.

J. L. Mueller and R. W. Austin. Ocean optics protocols for SeaWiFS validation, revision 1. In S. B. Hooker, E. R. Firestone, and J. G. Acker, editors, SeaWiFS Technical Report Series, volume 25, pages 1-67. NASA, 1995.

J. L. Mueller, C. Pietras, S. B. Hooker, R. W. Austin, M. Miller, K. D. Knobelspiesse, R. Frouin, B. Holben, and K. Voss. Volume II: Instrument Specifications, Characterization and Calibration. In J. L. Mueller, G. S. Fargion, and C. R. McClain, editors, Ocean Optics Protocols For Satellite Ocean Color Sensor Validation, Revision 4, chapter Instrument, pages $1-57.2003$.

P. R. Nair and K. K. Moorthy. Effect of physical properties of atmospheric aerosols on path radiance. Atmospheric Research, 43(2):139-155, 1997. ISSN 01698095. doi: 10.1016/ S0169-8095(96)00025-7.

M. Newberry. Tech Note: Pixel Response Effects on CCD Camera Gain Calibration. https: //www.mirametrics.com/tech_note_ccdgain.php.

L. G. Olmanson, P. L. Brezonik, and M. E. Bauer. Airborne hyperspectral remote sensing to assess spatial distribution of water quality characteristics in large rivers: The Mississippi River and its tributaries in Minnesota. Remote Sensing of Environment, 130:254-265, 2013. ISSN 00344257. doi: 10.1016/j.rse.2012.11.023. URL http://dx.doi.org/10. $1016 /$ j.rse.2012.11.023.

J. Orcutt, editor. Monitoring Earth System System. 2012. ISBN 9781461456834. doi: 10.1007/978-1-4614-5684-1_1.

J. E. O'Reilly, S. Maritorena, B. G. Mitchell, D. A. Siegel, K. L. Carder, S. A. Garver, M. Kahru, and C. McClain. Ocean color chlorophyll algorithms for SeaWiFS. Journal of Geophysical Research: Oceans, 103(C11):24937-24953, 1998. ISSN 21699291. doi: 10.1029/98JC02160.

R. E. O'Shea, 2020. A MATLAB version of the simulation framework is available for interested parties, contact the corresponding author (ryanoshea1@my.uri.edu) for access.

R. E. O'Shea and S. R. Laney. Simulation framework for evaluating lightweight spectral cameras in drone-based aquatic sensing applications. Applied Optics, 59:C52-C62, 2020.

R. E. O'Shea, S. R. Laney, and Z. Lee. Evaluation of glint correction approaches for fine-scale ocean color measurements by lightweight hyperspectral imaging spectrometers. Applied Optics, 59:B18-B34, 2020.

Z. W. Pan and H. L. Shen. Multispectral image super-resolution via RGB image fusion and radiometric calibration. IEEE Transactions on Image Processing, 28(4):1783-1797, 2019. ISSN 10577149. doi: 10.1109/TIP.2018.2881911. 
W. S. Pegau, D. Gray, and J. R. V. Zaneveld. Absorption and attenuation of visible and near-infrared light in water: dependence on temperature and salinity. Applied Optics, 36(24):6035-6046, 1997. ISSN 0003-6935. doi: 10.1364/AO.36.006035. URL https: //www . osapublishing.org/abstract.cfm?URI=ao-36-24-6035.

E. T. Peltzer. lsqfitgm, https://www.mbari.org/index-of-downloadable-files/. 2016.

R. M. Pope and E. S. Fry. Absorption spectrum (380-700 nm) of pure water. II. Integrating cavity measurements. Applied Optics, 36(33):8710-8723, 1997. URL http: //www.opticsinfobase.org/abstract.cfm?URI=ao-36-33-8710.

R. W. Preisendorfer. Hydrologic Optics: Volume I. Introduction. In Hydrologic Optics. 1976.

S.-E. Qian. Optical payloads for space missions. 2015.

S.-E. Qian. In Hyperspectral Satellites and System Design. Taylor and Francis Ltd., 2020.

Qimaging. Product Datasheet: Rolera-MGi PLUS High-Speed, Extremely Sensitive Digital EMCCD Camera.

P. Rakwatin, W. Takeuchi, and Y. Yasuoka. Stripe noise reduction in MODIS data by combining histogram matching with facet filter. IEEE Transactions on Geoscience and Remote Sensing, 45(6):1844-1855, 2007. ISSN 01962892. doi: 10.1109/TGRS.2007.895841.

Resonon Inc. Pika L, https://resonon.com/pika-l-camera. .

I. S. Robinson. Discovering the Ocean from Space. 2010. ISBN 9783540244301. doi: 10.1007/978-3-540-68322-3.

A. Roder. Slantrange, Inc., 4901 Morena Blvd, Suite 701, San Diego, CA 92117 (personal communication, 2019).

T. Sankey, J. Donager, J. McVay, and J. B. Sankey. UAV lidar and hyperspectral fusion for forest monitoring in the southwestern USA. Remote Sensing of Environment, 195: 30-43, 2017. ISSN 00344257. doi: 10.1016/j.rse.2017.04.007. URL http://dx.doi.org/ $10.1016 /$ j.rse.2017.04.007.

R. W. Sawtell, R. Anderson, R. Tokars, J. D. Lekki, R. A. Shuchman, K. R. Bosse, and M. J. Sayers. Real time HABs mapping using NASA Glenn hyperspectral imager. Journal of Great Lakes Research, 45(3):596-608, 2019. ISSN 03801330. doi: 10.1016/j.jglr.2019. 02.007. URL https://doi.org/10.1016/j.jglr.2019.02.007.

O. G. Sezer and Y. Altunbasak. Super-Resolution Reconstruction of Multichannel Images. In P. Milanfar, editor, Super-Resolution Imaging. CRC Press Taylor \& Frtancis Group, 2018. ISBN 9781439819319.

a. E.-S. M. Shaaban, H. A. Mansour, and A. A. Saber. Unveiling algal biodiversity of El-Farafra Oasis (Western Desert, Egypt ) and potential relevance of its use in water bioassessment: Special interest on springs and drilled wells. Egyption Journal of Phycology, 16(June 2015):47-75, 2015. 
T. H. Shahraiyni, M. Schaale, F. Fell, J. Fischer, R. Preusker, B. S. Shouraki, M. Tajrishy, H. Khodaparast, A. Tavakoli, T. H. Shahraiyni, M. Schaale, F. Fell, J. Fischer, and R. Preusker. Application of the Active Learning Method for the estimation of geophysical variables in the Caspian Sea from satellite ocean colour observations. International Journal of Remote Sensing ISSN:, 28:4677-4683, 2007. doi: 10.1080/01431160701442062.

S. Shang, Z. Lee, G. Lin, C. Hu, L. Shi, Y. Zhang, X. Li, J. Wu, and J. Yan. Sensing an intense phytoplankton bloom in the western Taiwan Strait from radiometric measurements on a UAV. Remote Sensing of Environment, 198:85-94, 2017. ISSN 00344257. doi: 10.1016/j.rse.2017.05.036. URL http://dx.doi.org/10.1016/j.rse.2017.05.036.

F. Sigernes, M. Syrjäsuo, R. Storvold, J. Fortuna, M. E. Grøtte, and T. A. Johansen. Do it yourself hyperspectral imager for handheld to airborne operations. Optics Express, 26 (5):6021-6035, 2018. doi: 10.1364/oe.26.006021.

Slantrange Inc. The All New 4P Series Sensors, https://slantrange.com/ product-sensor/.

A. Steer, A. Worby, and P. Heil. Observed changes in sea-ice floe size distribution during early summer in the western Weddell Sea. Deep-Sea Research Part II, 55(8-9):933-942, 2008. ISSN 09670645. doi: 10.1016/j.dsr2.2007.12.016.

T.-C. Su. A study of a matching pixel by pixel (MPP) algorithm to establish an empirical model of water quality mapping, as based on unmanned aerial vehicle (UAV) images. International Journal of Applied Earth Observation and Geoinformation, 58:213-224, 2017. ISSN 03032434. doi: 10.1016/j.jag.2017.02.011. URL http://linkinghub.elsevier. com/retrieve/pii/S0303243417300375

T. C. Su and H.-T. Chou. Application of Multispectral sensors carried on unmanned aerial vehicle (UAV) for trophic state mapping of the small reservoir in Kinmen, Taiwan. International Geoscience and Remote Sensing Symposium (IGARSS), 2015-Novem:5348-5351, 2015. ISSN 20724292. doi: 10.1109/IGARSS.2015.7327043.

M. Talone, G. Zibordi, I. Ansko, A. C. Banks, and J. Kuusk. Stray light effects in abovewater remote-sensing reflectance from hyperspectral radiometers. Applied Optics, 55(15): 3966-3977, 2016. ISSN 0003-6935. doi: 10.1364/AO.55.003966. URL https://www. osapublishing.org/abstract.cfm?URI=ao-55-15-3966.

Tetracam Inc. Macaw (MCAW) Tetracam's Multiple Camera Array Wireless System, http: //www.tetracam.com/Products-Macaw.htm. .

S. Totsuka, Y. Kageyama, M. Ishikawa, B. Kobori, and D. Nagamoto. Noise Removal Method for Unmanned Aerial Vehicle Data to Estimate Water Quality of Miharu Dam Reservoir, Japan. Journal of Advanced Computational Intelligence and Intelligent Informatics, 23 (1):34-41, 2019. ISSN 1883-8014. doi: 10.20965/jaciii.2019.p0034. URL https://www. fujipress.jp/jaciii/jc/jacii002300010034.

TriOS. RAMSES, https://www.trios.de/en/ramses.html. .

R. Y. Tsai and T. S. Huang. Multiple frame image restoration and registration. In Advances in Computer Vision and Image Processing, page 317-339. JAI Press Inc., 1984. 
U.S. Army UAS Center of Excellence. "Eyes of the Army" U.S. Army Roadmap for Unmanned Aircraft Systems 2010-2035. 2010.

L. J. V. Vliet, F. R. Boddeke, D. Sudar, and I. T. Young. Image Detectors for digital image microscopy. In M. H. F. Wilkinson and F. Schut, editors, Digital image analysis of microbes: imaging, morphometry, fluorometry and motility techniques and applications. Wiley, 1998.

K. Wang, B. Yong, X. Gu, P. Xiao, and X. Zhang. Spectral similarity measure using frequency spectrum for hyperspectral image classification. IEEE Geoscience and Remote Sensing Letters, 12(1):130-134, 2015. ISSN 1545598X. doi: 10.1109/LGRS.2014.2329183.

M. Wang. The Rayleigh lookup tables for the SeaWiFS data processing: Accounting for the effects of ocean surface roughness. International Journal of Remote Sensing, 23(13): 2693-2702, 2002. ISSN 01431161. doi: 10.1080/01431160110115591.

A. Watanabe and H. Furukawa. Super-resolution technique for high-resolution multichannel Fourier transform spectrometer. Optics Express, 26(21):27787, 2018. ISSN 1094-4087. doi: 10.1364/oe.26.027787.

J. Wei, Z. Lee, and S. Shang. A system to measure the data quality of spectral remotesensing reflectance of aquatic environments. Journal of Geophysical Research: Oceans, 121, 2016. ISSN 21699291. doi: 10.1002/2015JC011516.

P. J. Werdell, B. A. Franz, S. W. Bailey, G. C. Feldman, E. Boss, V. E. Brando, M. Dowell, T. Hirata, S. J. Lavender, Z. P. Lee, H. Loisel, S. Maritorena, F. Mélin, T. S. Moore, T. J. Smyth, D. Antoine, E. Devred, O. H. F. D'Andon, and A. Mangin. Generalized ocean color inversion model for retrieving marine inherent optical properties. Applied Optics, 52 (10):2019-2037, 2013. ISSN 15394522. doi: 10.1364/AO.52.002019.

K. A. Wójcik, R. J. Bialik, M. Osińska, and M. Figielski. Investigation of SedimentRich glacial meltwater plumes using a high-resolution multispectral sensor mounted on an unmanned aerial vehicle. Water (Switzerland), 11(11), 2019. ISSN 20734441. doi: $10.3390 / \mathrm{w} 11112405$.

S. B. Woźniak, M. Darecki, and S. Sagan. A practical method for estimating the light backscattering coefficient from the remote-sensing reflectance in the conditions of the Baltic sea and examples of its possible application." Preprint on https://essoar.org. 2018.

X. Xiong, K. Chiang, J. Sun, N. Che, and W. L. Barnes. MODIS on-orbit calibration: key issues and approaches. Proceedings of SPIE, 5542(May):24-34, 2004. ISSN 0277786X. doi: $10.1117 / 12.558191$.

J. Yang and T. Huang. Image Super-Resolution: Historical Overview and Future Challenges. In P. Milanfar, editor, Super-Resolution Imaging. CRC Press Taylor \& Frtancis Group, 2018. ISBN 9781439819319.

C. S. Yentsch. CZCS: Its Role in the Study of the Growth of Oceanic Phytoplankton. In V. Barale and P. M. Schlittenhardt, editors, Ocean Colour: Theory and Applications in a Decade of CZCS Experience, volume 53, pages 17-32. ISBN 9788578110796. doi: 10.1017/CBO9781107415324.004. 
J. A. Yoder, C. R. McClain, J. O. Blanton, and L. Oeymay. CZCS: Its Role in the Study of the Growth of Oceanic Phytoplankton. Limnology and Oceanography, 32(4):929-941, 1987. ISSN 19395590. doi: 10.4319/lo.1987.32.4.0929.

C. Zeng, M. Richardson, and D. J. King. The impacts of environmental variables on water reflectance measured using a lightweight unmanned aerial vehicle (UAV)-based spectrometer system. ISPRS Journal of Photogrammetry and Remote Sensing, 130: 217-230, 2017. ISSN 09242716. doi: 10.1016/j.isprsjprs.2017.06.004. URL http: //dx.doi.org/10.1016/j.isprsjprs.2017.06.004

G. Zhou, W. Xu, C. Niu, and H. Zhao. The polarization patterns of skylight reflected off wave water surface. Optics Express, 21(26):32549-32656, 2013. ISSN 1094-4087. doi: 10.1364/OE.21.032549. URL https://www.osapublishing.org/oe/abstract.cfm?uri= oe-21-26-32549.

K. Zhou, T. Cheng, Y. Zhu, W. Cao, S. L. Ustin, H. Zheng, X. Yao, and Y. Tian. Assessing the Impact of Spatial Resolution on the Estimation of Leaf Nitrogen Concentration Over the Full Season of Paddy Rice Using Near-Surface Imaging Spectroscopy Data. Frontiers in Plant Science, 9(July):1-18, 2018. ISSN 1664-462X. doi: 10.3389/fpls.2018.00964. URL https://www.frontiersin.org/article/10.3389/fpls.2018.00964/full.

H. Zhu, H. Liu, Y. Xu, and Y. Guijun. UAV-based hyperspectral analysis and spectral indices constructing for quantitatively monitoring leaf nitrogen content of winter wheat. Applied Optics, 57(27):7722-7732, 2018. ISSN 1559-128X. doi: 10.1364/AO.57.007722. URL https://www . osapublishing . org/abstract .cfm?URI=ao-57-27-7722.

L. Zhuang and J. M. Bioucas-Dias. Fast Hyperspectral Image Denoising and Inpainting Based on Low-Rank and Sparse Representations. IEEE Journal of Selected Topics in Applied Earth Observations and Remote Sensing, 11(3):730-742, 2018. ISSN 21511535. doi: 10.1109/JSTARS.2018.2796570.

G. Zibordi, M. Talone, K. J. Voss, and B. C. Johnson. Impact of spectral resolution of in situ ocean color radiometric data in satellite matchups analyses. Optics Express, 25(16): A798, 2017. ISSN 1094-4087. doi: 10.1364/oe.25.00a798. 